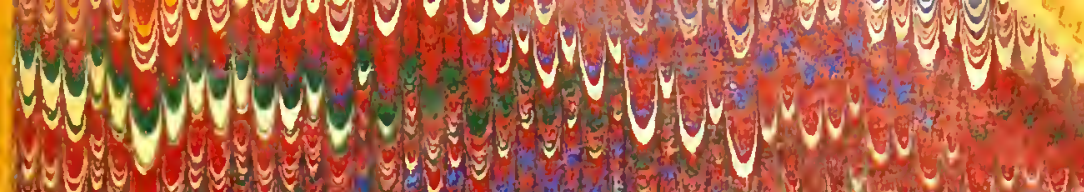

a 4

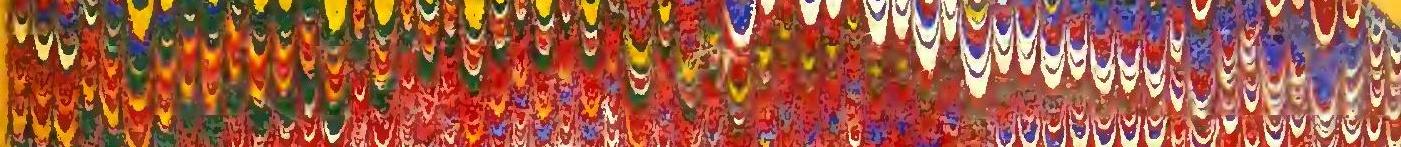

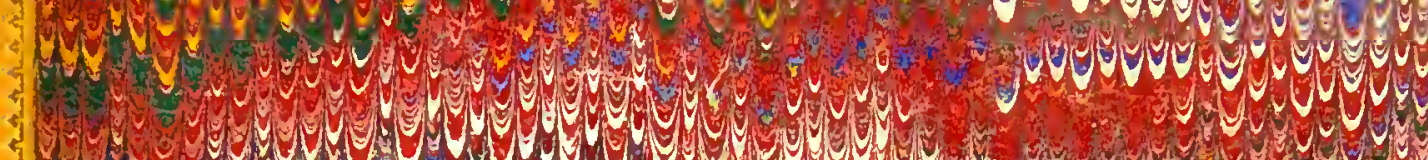

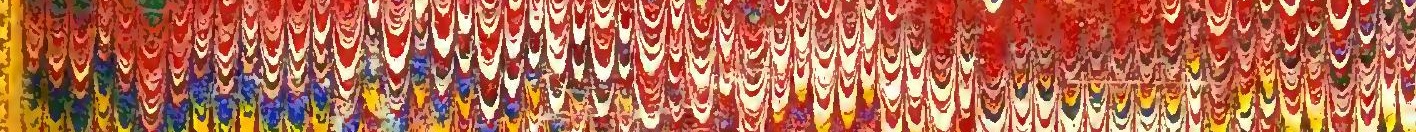
3.

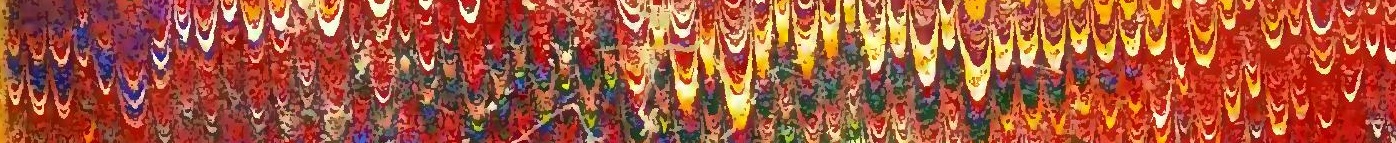
s.

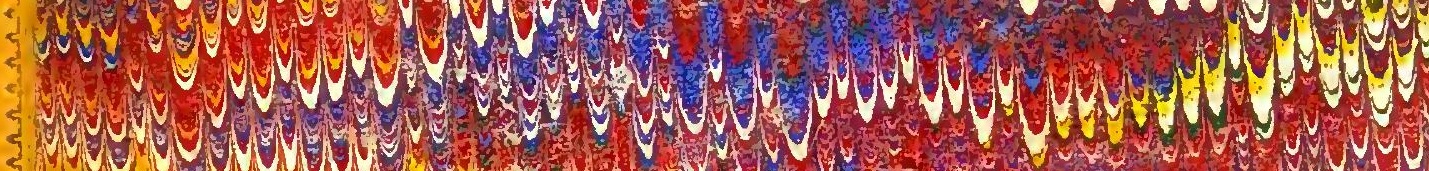
- Now 3.t. mover.t.

3.

. . . . 1.4. 3.

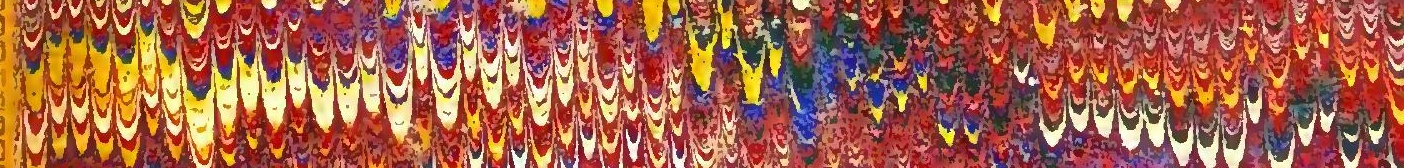

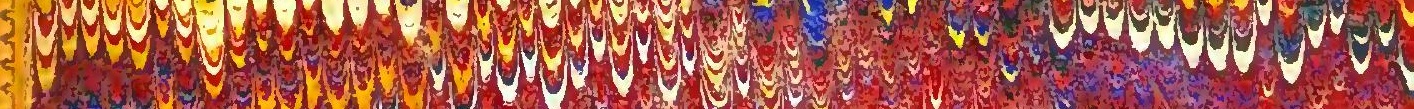

1. W w M w

y

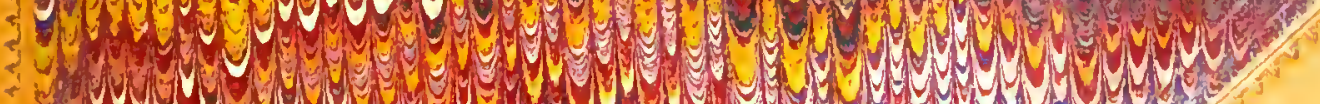
a y a 
beb. 35

$R 32170$ 




.





\section{T R A T T A T 0}

DELLE

\section{FUNZIONI RIPRODUTTIVE}

E

\section{D' EMBRIOLOGIA}

DEI,

\section{D. ${ }^{r}$ GIUSEPPE ALBINI}

PROFESSORE DI FISIOLOGIA NELLA R. UNIVERSITÁ DI NAPOLI MLEBRO ORDINARIO DELLA R. SOCIETÀ DELLE SCIENZE, eC. EC.

\section{N I P 0 L I}

STA BILIMENTO TIPOGRAFIGO VITALE

4 - Strada Pisanelli a Regina Coeli

1868 



\section{IND I CE ALPABETICO}

(il numero arabo indica la pagina del testo ed il romano la tavola dell' allante) 



\section{INDICE ALPABETICO}

a

Abbozzo del corpo . . . . . . PAg. 67 Tav. XI Allantoide. . . . . . . . . . . . " 74 " XI

Amnios e liquido amniotico . . . . . ) 77 " XI-XII Apparecchio genitale della donna . . . . " 37 " VI-VII Apparecchio genitale maschile . . . . . » 27 " IV-V Apparecchio vascolare e circolazione del feto nei

varii periodi della vita intrauterina . . . /) 117 „ XVIII-XIX. Archi e fessure branchiali . . . . . „ 92 " XV Area germinativa . . . . . . . . . " 64 " IX-X

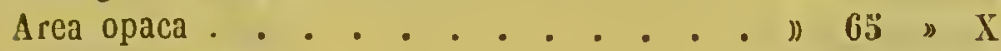
Area trasparente . . . . . . . . ॥ 65 „ X Area vascolare . . . . . . . . "11S " XVIII Arterie elicine . . . . . . . . . . . " 33 " V Alteggiamen to e posizione de! felo nell'utero . „144 „Aura seminale . . . . . . . . . " 23 " -

\section{B}

Blastoderma. $63 "$ IX

$\mathbf{C}$

Cambiamenti dell'uovo dopo l'uscita dal follicolo ») 59 " IX Caput gallinaginis . . . . . . . . . „) 34 " IV Circolazione del feto prima della nascita. . . ) 136 „ XVIIL-XIX Circolazione dell'allantoide e della placenta. . "129 „ XVIII Corda dorsale e vertebre primitive. . . . . " 69 " X-XII Corpi cavernosi del pene . . . . . . " 32 " IV Corpi del Wolfr. . . . . . . . . . . 074 ” XII Corpo luteo. . . . . . . . . \# 52 „ VI-VII

D

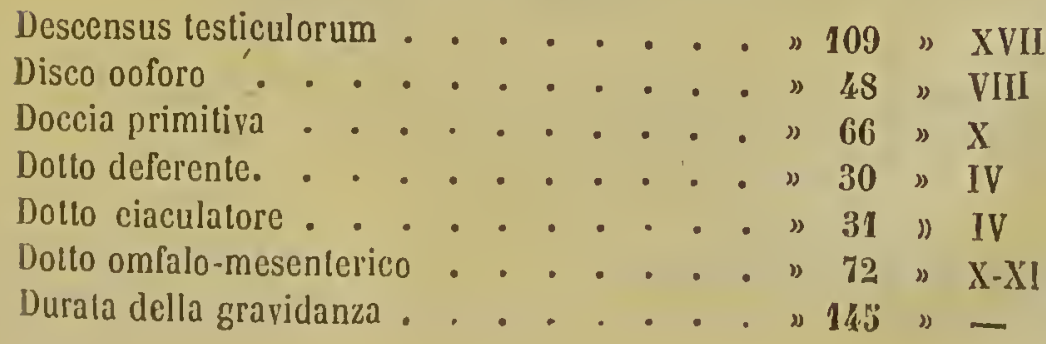


Epoca degli amori. . . PAg. 51 TAV. Ermafrodilismo. $16 "$ III

I)

Facollà riprodulliva . . . . . . . . " 8 "

Facolta riprodulliva dell'uomo . . . . . . " 25 " -

Fecondazione estcrna. . . . . . . . . .

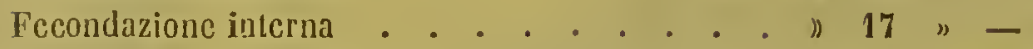

Follicolo del Graaf . . . . . . . . " 17 "VII

Formazione del sccondo e terzo cliorion (sicroso e vascolare) . . . . . . . . . " 73 " $\mathrm{X}-\mathrm{XI}$-XII

Formazione della membrana decidua (vera, rifles-

sa c scrolina). . . . . . . . . " 73 " XI-XII

Funicello ombelicalc. . . . . . . . . " 131 "XIX

Gencrazioni alternanti. . . . . . . . " 14 " -

Generazione equivoca . . . . . . . "

Gencrazione inegualc o differente . . . . . . " 9 " -

Gencrazione spontanca 0 primiliva . . . . . " 9 " -

Gravidanza, suoi segni . . . . . . . . . " 139 " -

Gravidanza cxtrauterina . . . . . . . . " 57 "

L.

Lallazione . . . . . . . . . . . " 149 " -

Locliii . . . . . . . . . . " $148 "$ -

Luogo ove i filamenti spermatici incontrano gli ovuli " 56 " -

\section{M}

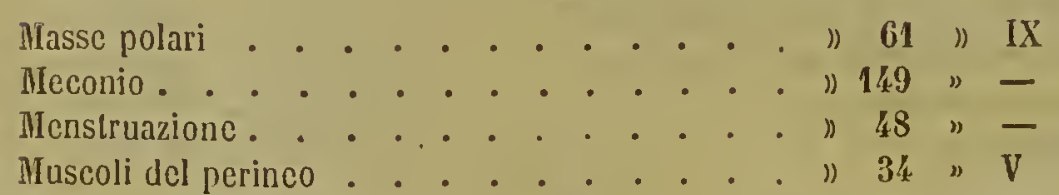

N

Neonalo

Nervi erigenli

Nola primiliva

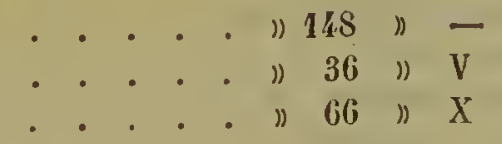

(1)

Origine primiliva degli organismi.

$8 \|-$

Ovaia, sua strullura

37 ) VI-VII 
Oripari

PAG. 17 TAV. -

Oro-vivipari.

$17)-$

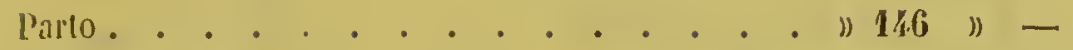

Parlenogenesi . . . . . . . . . „) 14 》 II

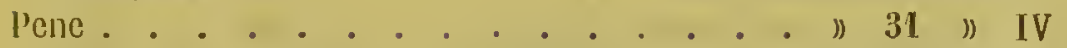

l'acenta felale c materna, loro struttura. . . " 131 » XVIII

Placenta e sue varielà negli animali . . . . ) 75 » XI-XII

Prima formazione dell'intestino e delle vescicola ombelicale. • . . . . . . . . " 71 .

Primo chorion villoso. . . . . . . . . . . 63 ) IX

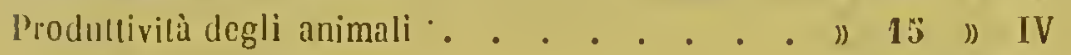

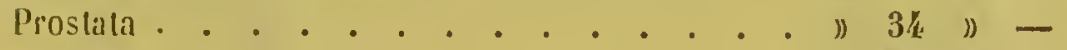

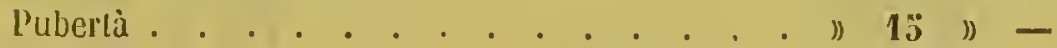

\section{Ie}

Rapporto dei scssi per la fecondazione . . . . " 530 ”

Riproduzione degti animali (Generalità). . . . " \$ " -

Riproduzione per gemme. . . . . . . . . . „ 13 ")

Riproduzione per germi (Uova Spore). . . . . " 13 " II

Riproduzione per scissione . . . . . . . . " " 12 " I

Segmentazione della massa vitellina . . . . " 62 „ IX

Sessiparilà . . . . . . . . . . " 16 " 16

Sperma . . . . . . . . . . . . \# 21 „ VIII

Stratificazione del blastoderma in tre foglielli . . " 63 " $\mathrm{X}$-XI

Superfecondazione . . . . . . . . . " 59 "

Superfelazione . . . . . . . . . . .

Sviluppo dei corpuscoli spermatici . . . . . „ 42 „ VIII

" Icgli ovuli nclle ovaic e loro uscila . . " 47 i VII

" dei singoli sistemi. . . . . . . . . " 79 "

" Uel sistema nervoso centrale e periferico. \# 79 " XIII

" delloscheletro (colonna vertebrale e cranio)" $8 \%$ " $8 \mathrm{XIV-XV}$

" della faccia. . . . . . . . " 91 " $91 \mathrm{~V}$

" degli arti . . . . . . . . . . .

" tlel tubo gastro-enterico e suoi annessi . " 94 ") XVI

" della lingua, denti, glandule salivari e muciparc . . . . . . . . . . . „ 97 „ XVI del fegato . . . . . . . . . " " del pancreas c delle glandule intestinali. 
Sviluppo del peritoneo ....... Par. 101 TAV. XVI

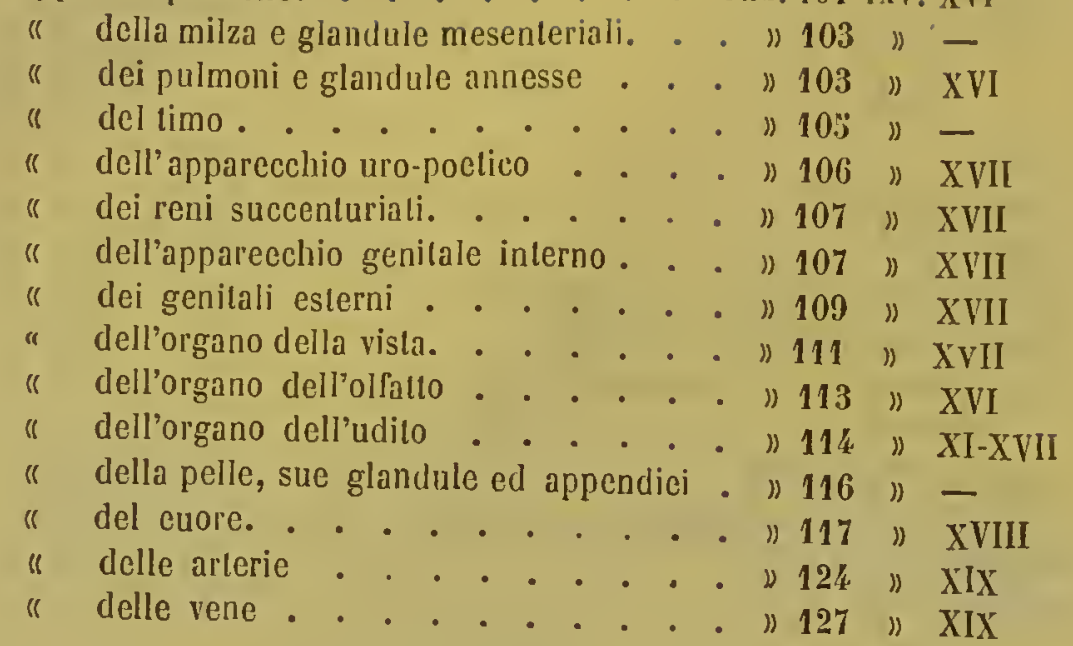

T

Teoria di Darwin . . . . . . . . . " " 11 "

Testicoli, loro invogli e stlultura . . . . . .

Trombe faloppiane.

Uovo degli uccelli. . . . . . . . . . " " 18 ॥ 111

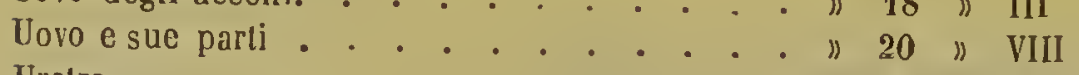

Uretra . . . . . . . . . . » 31 » IV

Utero . • • • . . . . . . . . . .

Vasi e nervi dell'apparecchio genitale maschile $\begin{array}{lllll} & 35 & \text { } & \end{array}$

Vasi e nervi dell'apparecchio genitale femminile .

Vescicola ombelicale... . . . . . " 72 " XI

Vescicole spermaliche . . . . . . . .

Vivipari semplici e vivipari mammiferi

T.

Zigosi. . . . . - . . . . . . . " 13 " II 
PARTE TERZA 

T R A T T A T 0

DELLA

VITA RIPRODUTTIVA 



\section{GENERALITÀ}

SULLA RIPRODUZIONE DEGLI ANIMALI

Tutti gli esseri organici nascono, crescono e muojono. Qualunque sia la durata della vita degli individui organici, ore o secoli, tutti hanno per comune carattere la temporaneità.

A rigor di termine questo carattere non è esclusivo ai duc regni organici (animale e vegetale); dappoichè noi vediamo come le influenze o violenze esterne, quali sarebbero i venti, le acque, i tremuoti, le cruzioni vulcaniche, le ghiacciaje perpetue, le correnti d'acqua epi-ed-ipogee, il flusso ed il riflusso del mare, la mano dell'uomo ed infine la vita assimilatrice degli organismi tutti, mutino sito e natura alle terre, alle roccie, ai minerali; dei quali il geologo segna le epoche di formazione, la durata dell'incremento, quella d'involuzione e di scomparsa, ossia un periodo d'esistenza con determinate proprietà fisiche e chimiche, che fanno distinguere un minerale dall'altro.

Per tanto sotto questo punto di vista fra individui organici e minerali non corre altra differenza che quella del tempo, ere od epoche per questi, ore o secoli per quelli. 
Ma l' inerzia delle molecole che costituiscono il minerale, le condizioni date dai rapporti che hanno fra loro e con quelle dei corpi coi quali stanno in contatto, rendono la durata del minerale esclusivamente dipendente dagli agenti esterni, mentre lo stato d' incessante movimento interno molecolare ed atomistico, proprio agli organismi (che si manifesta con fenomeni di sviluppo, di calorico, di moto, di luce, d'elettricità, di produzioni chimiche) fa sì che la vita di questi sembri dover cessare d' ordinario per cause intrinseche, ed invece le violenze esterne si considerino come cause eccezionali.

Chi fa professione di materialismo avrebbe molto a dire in proposito; ma io per ragione di brevità metterò fine ad una questione che non mena ad alcun risultato definitivo, qualunque sia l'arsenale filosofico che fornisca le armi ai combattenti.

Sembra che per regola la morte naturale degli animali debba avvenire per cause intrinseche, in quanto che la vita, come suol dirsi, porta in sè il germe della morte; ad una certa età $i$ tessuti e gli organi si consumano continuamente e non vengono reintegrati in tutto, ma sempre con un deficit quantitativo e qualitativo; sommandosi questi minus si ha il deterioramento or ganico, che limitato da principio anclie ad un solo tessu to od organo(per esempio al dente)influisce più o meno direttamente sugli altri,finchè fattosi generale cessano le funzioni e la vita. I continui processi vitali (chimici e fisici) sono sorgenti sicure di principii destinati ad essere eliminati, perchè improprii o deleterii (per esempio acido carbonico, urea, acido urico, sali) i quali non venendo eliminati nella proporzione in cui si formano finiscono coll'alterare le funzioni, col distruggere la vita (1).

La morte cosi detta fisiologica, o per vecchiaja, è un fatto troppo ovvio per metterla in dubbio onde distruggere questa

(1) "Depositi calcarei nelle pareti delle arteric e specialmente alle valvole dei vasi maggiori, atonia muscolare, alterazione dei succhi digerenti elc." 
differenza clie l'uomo mette quasi per istinto tra organismi e minerali.

Peró la vita del maggior numero degli organismi viene il più dellevolte troncata da influenze eslerne, che per se stesse sono distruttrici, e che abbiamo vedute capaci di rendere temporanea l'esistenza specifica degli stessi corpi inorganici (1).

Qualunque sia l'estensione che si voglia dare al significato della parola morle nalurale, il periodo dell' esistenza individuale sempre una frazione minima, trascurabile in confronto di quello della rispeltiva specic. Ma siccome la vita della specie non può considerarsi se non costituita da quella degli individui ne nasce la neccssità del loro riunovamento. E questo potrebbe verificarsi in differenti modi, cioè

$1^{0}$ per origine spontanea (Creazione)

$2^{\circ}$ per origine ineguale (Metembiosi)

$3^{\circ}$ per produttività degli organismi esistenti (Generazione).

E poichè gli organismi contemporanei all'uomo non hanno ancora fornito un fatto incontrastabile di alcuno dei due primi modi di rinnovamento, ne viene che, per la temporaneità degli individui, si renda necessario che $\mathrm{i}$ rispettivi organismi animali e vegetali sieno dotati della facollà di assimilare dal mondo esterno non solo la quantità di materiale necessaria a reintegrare il loro corpo delle perdite che continuamente subisce, ma ben anco una porzione eccedente, che in esso si plasmi e dopo un ciclo di melamorfosi entro o fuori del corpo stesso assuma infine le forme del generatore.

Una porzione dell'alimento serve pertanto a conservare la vita dell'individuo, l'altra a conservarne la specie. É così è che, fatte alcune poche eccezioni, gli animali più fecondi consumano anche grandi quantità d'alimento ed hanno un apparecchio digerente assai sviluppato, Di ciò ne abbiamo un esempio molto evidente rella gallina domestica, la quale

(1) Fra queste influenze cstcrne giova spccialmentc quil ricordare come il regno vegetale venga usufruttato dagli animali, c come fra questi gli crbivori scrvano d'alimento ai caruivori. 
síccome fornisce la maggior quantità di materiale di riproduzione rappresentato dalle uova, ha, secondo le mic dirette osscrvazioni, un tubo digerente lungo quasi il doppio di quello del gallo; c cosi anche l'ingluvic, i due ventricoli, il fegato, il pancreas, la milza sono assai più voluminosi nella femmina che nel maschio. La gallina inoltre essendo assai vorace non c̀ tanto difficile nella scelta dell' alimento come lo è il maschio.

E pertanto ogni specic organica avrà almeno alcuni individui, i quali per tutta la loro vita o durante un periodo di essa ci offriranno oltre i fenomeni derivanti dai processi organici ed animali, anche quelli della riproduzione o generazione, il di cui risultato finale consiste appunto nella produzione di nuovi individui, cioc̀ la conscrvazione della forma tipica organica mediante una seric d'individui mortali prodotti gli uni dagli altri.Questa proprictà degli organismi dicesi $\mathrm{Fa}$ coltà riprodultiva.

Ciò posto sorge la questione se una tal legge, che ormai sembra comunc ed invariabile, soffra od abbia soffertc delle eccezioni per individui di specic già esistenti, e se è possibile che anche oggidi sorgano nuove specie organiche?

E una necessità della mente umana l'ammettere una origine primitiva di esseri organici sulla superficic della terra. Come noi dotati di cinque sensi non possiamo concepirne un sesto, come il cieco nato non si formerà mai un'idea di luce c di colore, cosi i limiti della nostra intelligenza non ci permettono di spingere il pensiero in un passato illimitato, ma ci costringono a porvi un termine iniziale. Ma anclıc senza di cio, le scoperte scientiche geologiche provano con tutta l'evidenza che in un'epoca assai lontana da noi la superficic del nostro piancta cra incandescente c circondata da una fuliginosa atmosfera, quindi mancante delle condizioni indispensabili per essere vestita di vegetali o popolata da animali. Perciò, come abbiamo già detto, sorge necessaria l'idea di origine priniliva di organismi da materia inorganica, che la favola e 
la religione hanno avuto sempre in mira di far corrispondere a creazione dal nulla.

Ora questa origine primiliva, che pur si è dovuta ammettere necessariamente, non deve confondersi con la riproduzionc, la quale ne rappresenta la continuazione.

Però noi vediamo come molti naturalisti e filosofi speculativi di ogni epoca, scblene non potessero negare la riproduzione della maggiore parte degli animali secondo la legge da noi cnunciata, pure o sedotti o seducenti al meraviglioso, credettero (e credono) che individui di specic già esistenti potessero sorgere per origine primitiva, spontanea, equivoca od ineguale; espressioni tutte, che d'ordinario vengono considerate come sinonime. Noi per altro troviamo che vi sieno diffcrenze essenziali tra queste diverse cspressioni, ed intendiamo per generazione spontanea o primitiva la produzione d'individui organici dal connubio di elementi materiali inorganici. Per generazione equivoca la produzione d'individui animali o vegetali dal detrito di organismi morti, ed infine per generazione ineguale o differente la produzione di un organismo da un altro di diversa specie, genere., famiglia o regno.

L'autorità del padre delle scienze naturali, Aristotele, in- . flui grandemente a diffondere ed a fare ritenere quasi per certo che molte specie animali si originasscro eccezionalmente o dalla materia inorganica, o dal detrito di organismi e dei loro prodotti.

Percio il grande filosofo osservatore, la cui vita ed opere furono sprone e guida ai naturalisti che seguirono, ha sancito con la scienza dei suoi tempi alcuni errori, che vennero poi tolti non già da scolari che "jurant in verba magistri » ma da coloro che rispettosi ammiratori di tant'uomo ne perpetuarono il culto col sorregerne gli crrori, seguendone con sana critica l'csempio ed i precetti nell'osservare ed indagare le natura ed i suoi fenomeni.

Fra questi si distinsero specialmente Redi e Valisneri, 
dimostrando ambedue con prove di fatto e sperimentali erronea la credenza antica espressa dal motto:

Norine vides, quaecunque mora, fluidoque calore

Corpora tabescunt, in parva animalia verti?

Gli errori di Aristotele corretti da Redi, Valisneri, Spallanzani e molti altri riguardano animali di facile osservazione e non microscopici, quali sarebbero ranocchi, pesci, vermi, insetti ecc., intorno ai quali nessuno più si attenta di negarne la produzione da genitori affatto simili. Col progresso della scienza, colla scoperta del microscopio e di altri strumenti d'osservazione, non chè pei ripetuti esperimenti, la fauna degli animali che una volta dovevano riprodursi per generazione spontanea, equivoca od ineguale, andò mano mano riducendosi, sicchè oggidì la questione è discesa agli organismi nuicroscopici ; questione la quale non vuol dirsi certamente oziosa, facendo sorgere nuovi dubbii e menando a scoperte utilissime, fra le quali vorremo citare la possibilità di vita degli organismi o dei germi in talune condizioni di temperatura, di atmosfera ecc., che prima sembravano improprie, e di più la esistenza di larve affatto diverse dai loro genitori, che secondo alcune recenti scoperte sono in taluni casi perfino suscettibili di riprodursi, talchè veramente si hanno successioni di generazioni di stati larvali.

Dietro queste considerazioni noi siamo d'avviso che la legge ammessa incontestabilmente per tutti gli animali vertebrati e per gli invertebrati visibili ad occhio nudo, valga anche per gli organismi animali microscopici, e che la credenza d'una generazione spontanea o primitivadi questi sia mal fondata sopra difetto di osservazione, dipendente dalla imperfezione dei nostri strumenti e dei sensi, non che dalla ignoranza delle condizioni estrinseche necessarie o possibili per la couservazione e sviluppo di tutti gli organismi. E cosi a mò d'esempio, non si è mai pensato dai sostenitori della generazione equivoca o spontanea delle monadi, bacterii, ccc. che i germi di 
questi organismi ancorchè dotati di dimensioni visibili, potrebbero essere invisibili come un disco di vetro rell'acqua, per arere un’indice di rifrazione di luce eguale a quello del liquido in cui sono sospesi.

Ci rimane ora la questione della generazione ineguale, la quale venne dal Darwin applicata a tutti gli organismi e resa quasi insensibile, perchè secondo la sua teoria ha incominciato coi primi organismi (e forse con un solo primo organismo) e con gradazioni che si avvertono solo agli estremi si è continuata coll'andar dei secoli e probabilmente si continua anclie adesso.

Darwin ragiona nel modo seguente. "I figli assomigliano ai loro genitori naturali, ma presentano tra loro delle differenze, le quali si ereditano dai figli e si perpetuano con le generazioni. Ora queste lievi differenze di forma e d'aspetto pongono le generazioni in condizioni diverse nel vincere la lotta continua che devono sostenere per la conservazione degli individui e della specie; talchè i relativamente più deboli soccombono, e si conservano i più forti. Ripetendosi continuamente e col lungo andar dei secoli le differenze di forma nelle generazioni seguenti, per le quali variano appunto le condizioni della vita, come sarebbero la temperatura, la qualitá del suolo l'alimento, il convivere con altri animali ecc., le varietà subiscono delle variazioni successive nello stesso senso, al punto da divenire affatto differenti dal loro genitore, e cosi si passa gradatamente e quasi insensibilmente dalla formazione della varietà a quella della specie, del genere ec.

Menerebbe troppo per le lunghe il voler entrare in discussione sopra una tale teoria, che io ho esposta non già perchè sia affatto nuova (1) ma perchè Darwin la fece piuttosto rivivere appoggiandola a molti fatti raccolti con grande perspi-

(1) II molto di Linnco - nalura non facil saltus - veniva appunto interpetralo da molti nel seuso della teoria di Darwin. 
cacia e con ragionamenti assai ingegnosi ; ma tali fatti e ragionamenti non bastano per altro a sedurci per questa gretta iuterpretazione delle forze di natura ed a procurarci la convinzione che l'uomo, l'elefante, il cedro del Libano, e la vorticella riconoscano in un filozoa il loro comune progenitore.Dap. poichè senza rinunciare al buon senso si ponno ammettere delle condizioni e delle forze diverse contemporanee o pure verifcatesi in tempi fra loro lontani, alcune delle quali diedero origine al filozoa del Darwin ed altre al Re della natura.

Gli animali si riproducono per diversi modi, cioè : per Scissione, per Gemme, per Germi. Gli animali superiori hanno un sol modo di riprodursi, mentre gl' inferiori si riproducono in diverso modo secondo le condizioni in cui vengono posti. Questa facoltà degli animali inferiori avrebbe una spiegazione teleologica nella necessità di conservare le loro specie, clıe per la minutezza od esiguità delle forme e per debolezza naturale sono più esposte a violenze che tentano farle mutare condizioni di vita, e quindi a distruggerle.

Tutti gli animali ed anche l' uomo hanno la facoltà di riprodurre delle parti clie vengono staccate dal corpo per violenza od altre cagioni ; cosi l' uomo riproduce i peli, le unghie, l' epidermide, il tessuto connettivo, l'osso, il nervo, i vasi, e si laanno perfino casi di riproduzione della lente cristallina ecc. (1) Discendendo nella scala degli animali la facoltà riprodutliva di parti perdute va aumentando, talche noi vediamo riprodursi le estremità, la coda, gli organi dei sensi ecc., nei girini delle rane, nei tritoni, nelle lumache etc. Infine nei vermi ed in altri animali inferiori questa facolta di riproduzione è così grande che l'animale può essere ta-

(1) In ru cane operato di fistola biliare col metodo di Stackmann ho asservato la riproduzione del dotto coledoco. 
gliato in tanti pezzetti, ora normali al suo asse, ora parallelamente allo stesso, ed i singoli pezzelti si completano in individui, riproducendo alle estremità le parti perdute; talchò come l'agricoltore clie da lanti pezzi di radice o da tanti rami di un albero ottiene altreltante piante, così dai singoli pezzelti del verme, del lombrico o della planaria si hanno altrettanti iudividui. Questo ì il primo modo di riproduzione che abbiamo detto per scissione, ed in taluni animali sembra verificarsi spontancamente, come nelle vorticelle, nei paramecii sebbene molti falti di riproduzione creduti per scissione non sieno altro che fenomeni di zigosi (1) o di gemmazione. Pare però piu probabile che la riproduzione per scissione avvenga d'ordinario per cagioni violente anziclı̀ naturali.

La generazione per gemme consiste nella produzione all' esterno od all' interno del corpo dell' animale madrc di appendici simili al corpo generatore, le quali a poco a poco s'ingrandiscono e diventano non solo per aspetto, ma anche per dimensione simili alla madre. Queste gemme o rimangono per tutta la vila aderenti al corpo della madre, e producendone alla lor volta delle altre danno origine a colonic, oppure sc ne staccano una volta raggiunto un certo sviluppo e menano vita libera. Infine talvolta nell' interno del corpo dell' animalc si sviluppano molte gemme, le quali crescono a spese e danno del corpo della madre, che finalmente scoppia per dare uscita ai figli.

Viene in ultimo la generazione per germi, vale a dire la produzione di corpuscoli sferoidali cellulari nello interno del corpo della madre, i quali subiscono un ciclo di metamorfosi, compiuto il quale, il corpo cellulare si è tramutato in un animale simile alla madre oppure in un animale di forma diversa che dicesi larva. Questa larva poi verificandosi certe condizioni, assume le forme della madre, oppure produce altre larve, le quali poi in ullimo diventano simili alla prima genitrice.

(1) Zigosi. Saldamento di due animali in un solo. 
Il germe dicesi spora quando si sviluppa spontaneamente, cioc incomincia e percorre il ciclo delle metamorfosi, senza arer d' nopo di sentire alcuna inlluenza di altri liquidi o cellule animali ; ed in tal caso si Ira la Parlenogenesi; dicesi uovo quando per srilupparsi dere sentire l'irfluenza di altre ecllule, le quali diconsi fecondatrici, perche si formano nell' istesso organismo o pure in un altro e non hanno altro ufficio clic quello di comunicare ai germi l'impulso allo sviluppo.

Concludiamo quiudi che la vita degli individui è temporanea, e sulla temporanciti è fondato il fatto della riproduzione, la quale si può verificare in quattro maniere, cioè per Scissione, per Gemme, per Spore o per Uova. La riproduzione nei primi tre modi dicesi ancle agama, cioè senza sesso, e l'ultima dicesi sessuale, dappoichè tanto le cellule fecondabili che le fecondatrici vengono prodotte da due organi diversi, i quali negli animali superiori trorandosi separati in dirersi individui caratterizzano i due diversi sessi.

Non dobbiamo per altro credere cle esistano dei limiti assoluti fra l'uno e l'altro, anzi sembra cle sieno assai frequenti le gradazioni di passaggio fra i quattro differenti modi di riproduzione sopraindicati; tal che il naturalista rimane spesso dubbioso nello stabilirne la natura, uon sapendo ben determinare e distinguere, a mo'd'esempio, la gemmazione dalla seissione, la gemma dalla spora e questir dall noro.

Una seconda osservazione ci resta ancora a fare intorno a questo argomento ed $\ddot{e}$, che tutti gli animali vertebrati c moltissimi invertebrati si riproducono soltanto per uora, mentre che moltissimi animali inferiori, ancorche forniti della facoltì di riprodursi per uora, pure in certe fasi della loro vita (che debbono percorrere per necessilì, oppure per condizioni speciali create da circostanze fortuite) si riprodıcono nell uno o nell'altro dei tre modi di generazione agama, dando cosi origine a delle generazioni, le quali si riprodncono col rimnorarsi delle condizioni necessarie o castrali e che perció diconsi Generazioni allernanti. 
Gli animali d'ordinario lanno la facoltì di generare soltanto in un periodo della lor vita, che incomincia quando l'individuo ha raggiunto un tale sviluppo in dimensione c robustezza, in cui le eccedenze degli introiti, dapprima impiegate all' ingrandimento del corpo, possono essere riserbate come materiali di riproduzione. Per gli animali superiori, come per l' uomo, questa elà (puberlà) si rende manifesta non solo per lo sviluppo delle glandule e dell' apparecchio genitale, ma anclie per alcuni cambiamenti di tulto il corpo e di alcuni organi speciali, non che per l'istinto del sesso.

Si parla di grandi differenze di produttività degli animali, le quali sono veramente straordinarie se siriflette unicamente alla quantità di materiale di riproduzione che forniscono (1).

Le cifre per altro si riducono quando si tiene conto del numero d'individui che si formano da questo matcriale e più ancora se si calcolano sollanto quelli i quali raggiungono l'e tà in cui essi stessi dovranno riprodurre, cioè dopo di avere superati tutti gli ostacoli e resistito alle violenze distruttrici che incontrarono. Infiue bisogna anclie considerare la durata della vita dei diversi animali ; perchè in un certo periodo di tempo si conservi un dato numero d'individui d'ogni specie, quelli che vivono poco tempo dovranno naturalmente essere, come in fatti lo sono, di maggiore fertilità.

Lasciando all' Anatomia comparata ed alla Zoologia lo stum dio deltagliato dei tre primi modi di riproduzione agama, delle metamorfosi, delle generazioni alternanti ccc. noi ci limiteremo all'esame del quarto modo con cui si verifica la riproduzione degli animali, cioè alla generazione sessuale per mezzo di cellule fecondabili (uova) e cellule fecondanti (corpuscoli spermalici).

(1) Cosl se si calcola la produttivita degli animali dal rapporto fra il peso e volume del corpo e quello del prodotto di generazione, noi troviamo che la donna produce all'anno, in media, un quallordicesimo, la serofa la melà del proprio peso, la gallina cinque volle e l' ape centodicci volle il proprio peso. 
Tanto gli ovuli come i corpuscoli spermatici sono negli animali superiori il prodotto di un organo particolare destinato unicamente alla loro produzione, per cui quest' organo o appare nell' animale soltanto ad una data cpoca della vita, oppure, se esiste fin dalla nascita, rimane in uno stato rudimentale e d'apparente inerzia, fino a clie l'animale clie lo porta lia raggiunta quell'età che dicesi pubertà, la quale segna il momento in cui l'animale può riprodurre.

L'organo produttore degli ovuli dicesi Ovaia.

L'organo produttore dei corpuscoli spermatici o cellule fecondatrici dicesi Testicolo.

Questi due organi nei vertebrati ed in molti invertebrati si trovano d'ordinario distribuiti in diversi individui, i quali si distinguono altresi per altri caratteri particolari di forma, di dimensione, di robustezza, di colore dei tegumenti comuni e loro appendici, e infine anche per facoltà psichiche.

L'individuo che porta i testicoli dicesi maschio, e quello che porta le ovaie dicesi femmina.

Eccezionalmente invece in alcuni vertebrati (a preferenza nei pesci) e regolarmente in moltissimi invertebrati troviamo riunili questi due organi in un solo individuo, il quale vien dello ermafrodito. L' ermafroditismo puó essere poi completo od incompleto. Dicesi completo allorquando l'ovaia, il testicolo e gli organi conduttori dei loro prodolti trovansi in tali rapporti anatomici tra di loro da potersi verificare il reciproco contalto o fecondazione nell' interno stesso dello individuo, il quale come per tutte le altre funzioni, cosi ancle per la riproduzione della specic basta per tal modo a sè solo (autogamo).

L' ermafroditismo dicesi poi incompleto allorquando l'individuo, sebbene inunito dei due organi, per particolare disposizione anatomico-topografica degli stessi, non può fecondare le propric nova, ma deve unirsi con un altro indisiduo 
per fecondare o per essere fecondato; molte volte i duc individui si fecoudano reciprocamente e contemporancamente, altre volte invece, gli organi si sviluppano ill diverse epoclıe nei dirersi individui per cui, sebbene crmafroditi, rappresentano or l' uno or l' altro sesso.

La fecondazione delle uova negli animali a sesso distinto, ed auche in alcuui ermafroditi incompleli, può verificarsi o nell'interno del corpo dell'animale o pure all' esterno; nel primo caso, che diccsi fecondazione interna, è necessaria la copula o l'accoppiamento dei due individui, dei quali d'ordinario l'indiriduo maschio versa il liquido fecondatore sull'ovaia o meglio nel dotto che riceve gli ovuli da essa prodotti.

Gli animali a fccondazione interna si possono dividere in vivipari mammiferi, vivipari semplici, ovo-vivipari ed ovipari.

Gli ovuli fecondati nell' interno possono trattenersi nel corpo della madre per un certo tempo ed ivi svilupparsi e nutrirsi a spese della stessa, mettendosi più o meno presto in rapporto coi suoi vasi sanguigni, i quali scrvono a portare al feto l'ossigeno ed il materialc d'ossidazione necessario al suo incremento. Gli animali che si sviluppano in questo modo distinguonsi in vivipari mammiferi e vivipari semplici; i primi sono abbastanza noti, molto rari invece sono gli esempii dei vivipari semplici (Mustaelus levis, pesce cartilagineo).

Diconsi ovo-vivipari quegli animali a fccondazione interna, nei quali le uova si sviluppano nell' interno del corpo della madre e ne escono, come i vivipari, dopo di avere acquistate le forme di animale vero, scnza mettersi però mai in rapporto di continuità col corpo materno; nc abbiamo degli esempii in molti vertebrati (Salamandra maculata, alcuni serpenti) e negli invertebrati (Paludina vivipara).

In fine diconsi ovipari quegli auimali a fecondazione interna od esterna, i quali depongono i germi allo stato di uovo, d'ordinario munito di organi protettori e sempre ricco di materiale nutritizio; c questo è il modo forse più generale di ri- 
produzione degli animali esistenti attualmente (uccelli, quasi tutti i rettili, anfibii, pesci, insetti, aracnidi, molluschi ctc.)

"Quì vicno acconcia una breve digressione sull" uovo degli ueeelli ehe viene considerato come il tipo delle uova degli altri animali, e faeciamociò anche per dare un'idea dei molteplici mezzi ıaturali per eonservarle e tutelarle, non che per munirle del materiale necessario allo sviluppo, dal momento che i germi di questi animali abbandonano allo stato li uuvo il corpo materno.

L' uovo degli uccelli è rappresenta to da quella macchietıa bianca ehe ognuno può facilmente vedere nel tuorlo dell' uovo di questi a nimali ; questa maeehiatta appellasi calcar avis o cicatricola. E questo germe ò la prima parte dell' uovo che si sviluppa nell' ovaia. II tuorlo, che impropriamente viene chiamato vitello, si forma più tardi intorno all' ovieino, ehe a poco a poco si porta alla periferia del tuorlo; quest'ultimo è tutto circondato da una membranella esilissima che eopre e ehiude anche il calcar avis. Tutto questo suecede iil un saeeo peduneolato e vascolare detto follicolo, formato dallo stroma dell' ovaia, ehe in questi animali è impari ed ha l'aspetto di uu grappolo d' uva, i di eui acini sono di diversa dimensione. Il saeco dell' ovaia che contiene il tuorlo presenta, quando il tuorlo è arrivato ad un certo grado di sviluppo, una zona meridianale detta stigma, priva di vasi e molto sottile, la quale per l'ineremento del tuorlo si assottiglia sempre più e finalmente si lacera, lasciando cosi cadere il tuorlo nell' apertura imbutiforme ed a bordo frangiato dell' ovidutto. Il follicolo aperto, per retrazione dei margini versoil pedaneolo, aequista la forma di un ealice ehe a poeo a poeo si atrofizza e seompare. Caduto pertanto nell' ovidutto, il tuorlo lo pereorre dall' alto in basso girando sul proprio asse e circondasi di strati d'albume, che viene`segregato dalle innumerevoli gländule esistenti nello spessore della mucosa di questo eondol to (1).

Gli strati d'albume intorno al tuorlo sono diver:amente consistenti

(1) Essai probabile ehe l'albume non si formi altrimenti ehe per una melamorfosi albuminosa delle cellule epitcliali che taperzano la mucosa e le glandule, come in altre parti per metamorfosi di cellule abbiamo la produzione di altri principii albuminoidi, nuali saltebloro la cascina del latte, la mucina rlel inuco etc. 
e presentano delle membranelle omogenee concentrielıe con sepimenti normali ed obliqui; gli strati più centrali dell'allsume sono anehe i più densi, i perilurici più fluidi.

Il movimento progressivo e di rotazione del tuorlo nellovidutto spiega la lormazione di quei due cordoni contorti e bianchieei detti chala$\approx e$, che dai poli del tuorlo, cui sono fortemente aderenti, attraversano tulto l'albume e terminano eon un fiocco aneora più bianco, il qualo alla sua volta s' inserisec alla membrana testacea. Lo strato più ester-no di questa membrana si aditta in tutta la sua estensione al guseio ealeareo e poroso, che si firma per depositi di sali di calce intorno ad essa nella porzione inferioro dell' ovidutto (1). 'Lo strato interno della membrana testacea ad un polo dell' uovo (ordinariamente al polo ottuso) si stacca dallo strato esterno, lasciando eosi tra l'uno e l'altro uno spazio leutieolare piano-eonvesso che contiene aria, per cai vien detto anche camera d'aria.

Il guscio calcareo infine è coperto da una sottile e liscia culicula, clie dà al guscio dell'uovo la levigatezza ed il colore, perchè in essa si depositano i pigmenti ora dilfusi iu uno struto omogeneo ed ora raecolti in isolette 0 macehie $n$.

Già in alcuni anfibii e quasi generalmente nei pesci ed altri animali d'acqua noi troviamo la fecondazione esterna delle uova, le quali o vengono deposte dalla femmina presente il maschio, che talvolta l'aiuta a deporle comprimendone l'addome (Rana obstaetricans) e le feconda appena uscite; oppure le femmine depongono le uova in assenza dei maschi, i quali o vengono a fecondarle, oppure versano in altre località il liquido fecondatore clıe viene poi trasportato dalle onde. Questo fatto conosciuto da tempi antichissimi si utilizza anche oggidi per la fecondazionc artíficiale dei pesci e per popolare in tal modo i fiumi, i laghi, i seni di mare ecc. Questa industria vien detta piscicoltura.

(1) Il guscio calcarco la una basc organica della stessil natura dclla membrana testacea, colla qualc sta in un rapporto analogo a qucllo che si osserva fra l'osso ed il periostio; di modo che distruggendone coll' acido cloroidrico od acctico i sali calcarei, si vede distintamentc la conlinuita della memhrana testacea colla hasc organica del guscio. 
Come abbiamo osservato a proposito dei quattro modi di riproduzione, così non possiamo tacere anche ora rispetto alla fecondazione iuterna ed esterma delle uova, che non sempre esistono dei limiti assoluti e distinti; ma piuttosto rerificansi delle gradazioni di passaggio dall'uno all'altro, che meltono spesso in dubbio sulla definizione : tanto più che il modo di fecondazione viene qnalclie volta modificato da condizioni forluite.

DELLE SOSTANZE ESSENZIALI ALLA RIPRODUZIONE SESSUALE GIOÈ DELLE UOVA E DELLO SPERMA.

L'uovo si forma in un organo glandulare detto ovaia, ell è un corpuscolo sferoidale, una cellula, in cui si distingue un protoplasma granuloso, che dicesi vitello, contenente una vescicola trasparente detta vescicola germinativa, la quale alla sua volta contiene uno o più corpuscoli opachi detti macule germinative. In quasi tutte le uova il protoplasma o vitello ì circondato da una inembranella esterna detta membrana vitellina; quando questa membrana ha uno spessore tale da presentare un contorno esterno ed uno interno, come succede nell'uoro dell'uomo, di molti mammiferi ed altri animali, la i itellina prende anche il nome di zona pellucida, perchè veduta al microscopio, questa membrana omogenea spessa ed a doppio contorno si presenta come una zona pellucida ed omogenea circondante il proloplasma. Membrana vitellina adunque è il nome generico e zona pellucida è il nome specifico sebbene improprio, perchè involge lutto l' voro e non una sola zona dello stesso, di modo che'si vede sempre circondare l'uovo qualunque sia la posizione che si dà allo stesso.

La membrana vitellina talvolta è così esile che se ne mette perfino in dubbio la esistenza (per esempio nell' uoro di gallina) tal'altra è altraversata normalmente da tanti canaletti esilissimi (Talpa) detti pori canali, che mettono il, vitello in comunicazione coll'esterno; o pure si presenta a modo di una 
fiala, mel cui corpo si contiene il vitello (Oloturia, V'cnus) e con un collo che si apre superiormente a mó d'imbuto detto micropile; oppure infinc porta alla sua superficie libera Jelle ciglia o villositi clıc damno all'uovo un aspelto echinato.

Rigruardo alla composizione chimica degli ovuli può dirsi in generale cle il vilello consta di una miscela di sostanzc alluminoidi, di grassi, di idrati di carbonio, di sali ed acqua, comprendendo in tal modo tutti gli clementi e loro combinazioni cle costituiscono l' organismo animale. E cosi le uova della rana da me analizzate contenevano acqua, diverse sostanze albuminoidi, una sostanza mucosa, grassi, pigmenti o sostanze coloranti e sali.

Le uova di gallina constano di 77-78 parti per 0/0 di albune e $32-33$ per $0 / 0$ di tuorlo. Questo che è la parte esscuziale, cousta di

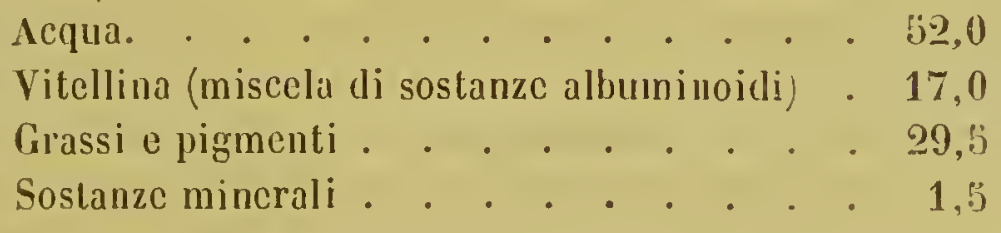

Fra i minerali prevalgono i fosfati cd i cloruri.

Di più tutte le uova contengono piccole tracce di sostanze estrallive, lc quali talvolla sono velenose per altri animali e per l'uomo ; questa proprietà può essere considerata come un'arme di difesa per tutelare la specie.

L'organo glandulare maschile per la riproduzione, detto testicolo, produce le cellule fecondatrici (corpuscoli spermatici), le quali giunte a perfezione o maturita abbandonano la glandula insicme ad un liquido albuminoso assai affine al plasma sanguigno, c percorrendo il dotto escrctore della grlandula gitungono all' esterno. D' ordinario le cellule fecondatrici sospese nel liquido nou vengono immediatamente versate all' esterno, ma si raccolgono in serbatoi particolari (specic di appendici cieche del (lolto escrelore) nei quali rimangono per un 
certo tempo e spesso si mescolano con altri umori segregati da glandule accessorie. Da ciò ne viene che pel consueto si parla di liquido fccondatore o sperma, in quanto clic le ccllıle fecondatrici rengono portatc all' uovo (critro o fuori del corpo della femmina) dal liquido o meglio dalla miscela di liquidi in cui sono sospesi. Soltanto in pochi animali invertebrati i corpuscoli spermatici conscrvano la forma di cellule munite di processi; più comuneınente invece questi corpuscoli si presentano sotto la forma di filamenti, con una estremita ingrossata detta corpo o lesla c coll'cstrcmità opposta assoltigliata detta coda. Nello sperma fresco questi corpuscoli o filamenti spcrmatici lianno un movimento che si assomiglia a quello delle anguille o vermi, poichè agitando la coda nel liquido si spingono in una data direzione coll'estremità ingrossala in avanti; ma il loro movimento non è continuo, e cosi purc si vedono cambiar spesso di dirczione e di vclocitì. (1) Questi fatti insicme all'aspetto particolare dei filamenti spermatici han fatto credere per molto tempo che fosscro piccoli animalctti, di modo che si appcllarono anche spermatozai; ma la mancanza di organi interni ed il modo di comportarsi rerso gli agenti estcrni e specialmentc con i rcagenti chimici c'inducono a metterli nolla categoria dellc cellule vibratili per semplice fenomeno di riffusione.

Sarebbe quasi inutile il ricordare che i filamenti spermatici dei diversi animali presentano delle differenze di dimensionc e di forma tanto dell' estrcmità ingrossata o testa, come del filamento o coda, e che acquistano questc forme specificlie soltanto allorchè sono perfettamente sviluppati.

Come è naturale qucsti corpuscoli sono la parte csscriziale del liquido fecondalore, talchè filtrando lo sperma, ralc a dire dividendo il plasma dai corpuscoli, il plasma filtrato posto in contatto delle uova, anche uclle condizioni piu farorevoli, non

(1) L'organo del movimento progressiyo dei corpuscoli spermatici è rerancnte la coda o filameuto, dappoichè dore manca vie soltanto oscillazioIIe, dove i l) reve il movimento í leuto. 
arriva mai a lecondarte. Vi fu un tempo in cui alcuni medici e naturalisti credevano ad an' attivita fecondatrice della così detta aura seminale, vale a dire di quel vapore d'ordinario odoroso clıe esala dallo sperma fresco. Le ricerche ingegnosissime dello Spallanzani provarono l'erroneita di questa esagerala opinione sull'attivilà fecondatrice dello sperma. Prevost et Dumas non solo confermarono gli esperimenti del Prof. di Pavia, ma provarono ancora che l'attivita l'econdatrice dello sperma stava ill proporzione diretta del numero dei filamenti spermatici in esso contenuti (1).

Lo sperma essendo un liquido animale assai denso, specialmente per la presenza di tanti corpuscoli, non perde molto in volume per l'evaporazione o disseccamento giacchì consta di $18-20$ p. 0 0 di residuo solido; $82-80$ p. 0 ! 0 di acqua.

Il residuo solido lia un colore bianco giallognolo, si può ridurre in polvere ed allora è untuoso al tatto e d' un odore differente nei diversi animali; abbrucia sviluppando forte odore empireumatico e lascia molto carbone o cencre. Il residuo solido consta per la massima parte di sostanze albuminoidi, alcune appartenenti ai corpuscoli ed altre al mestruo; vengono

(1) Le espericuze che io ho iutrapleso da qualche anno specialmente sulle galline allo scopo di provare quali condizioni potessero influire sulla determinazione del sesso, in luogo di coudurmi a qualche risultato soddisfacente mi fecero sorgere nuovi dubbii; però mi fornirono lioccasione di osservare alcuni fatti importanti, dei quali ne citerò nno che credo qui a proposito ed è il seguente. Le galline per dodici o quindici giorni dopo la uccisione o l'slloutanamento lel gallo depongono delle uova fecondate, ma le nova degli ultimi giorni, cioè quelle del dodicesimo al quindicesimo giorno d'ordinario inconninciano a svilupparsima abortiscono, cio che ci fa supporre uı fecondazione insufficiente dipendente dal poco numero di filameuti spermatici, o pii probabilmente ancora da esaurimento di forze degli stessi avrenuto nell'ovidutto della gallina, in cui dimorarono per tanti griorni, Le mic osservazioni e ricerclic nou mi lasciann quasi piu dubhio che nelle galline le uora non vengono mai focondate nell'ovaia; poiche la cicatricola uel tuorlo contenutonel follicolo é identica iu lutte le galline, mentre rhe la cicatricala delle nova deposte ed ancle di quelle che si trovano nell'ovilutto di galline isolate dal gallo si distingue, auclıe per caratteri macrosenpici, da quello delle nova di galline fecondate. 
ill seguito i grassi, le sostanze estrattive ed i sali, fra i quali predominano i fosfati, i solfati ed i cloruri alcalini.

Ora sorgono naturalmente le domande sulla sorte dei filamenti spermatici, oppure sul modo con cui questi fecondano le uova, sulla parte che prendono nel formare il nuovo animale e nel determinarne il sesso, ed infine sulle cause che determinano l'incontro dello sperma coll'uovo.

I fatti noti a questo riguardo sono pochissimi, ma pure sono sufficienti almeno a dimostrarci che la fecondazione si verifica unicamente pel contatto immediato d' uno o piu filamenti spermalici col protoplasma della cellula uovo, vale a dire col vitello. Nelle uova con micropile o con pori canali si osservarono piu volte i filamenti spermatici penetrare per quelle aperture nell'interno dell'uovo; e si è pure osservato che dal momento della penetrazione del filamento spermatico incominciano quelle metamorfosi, che lianno per risultato finale la formazione dell animale. Anche uelle nova di altri animali nelle quali finora non si sono trovate delle aperture (pori-canali o micropili) si osservarono i filamenti spermatici muoversi nell'albume o nel muco che circonda l'ovicino ed alcuni asscriscono d'averli veduti anche uell'interno dell'uovo. Bisogra quindi supporre o che queste uova sieno munite di aperture non ancora scoperte, oppure che i flamenti spermatici si facciano strada attraverso alla membrana vitellina, ciò che non sembra tanto fuori del possibile quante volte noi vediamo che altri flamenti organici esilissimi (organi urticarii) possono perfino perforare la pelle dell'uomo producendo il fenomeno dell'urticazione.

E quì vorremo arrestarci innanzi al vuoto immenso creato dalle domande sopra citate, anzichè tentare di riempirlo con una o coll' altra delle tante ipotesi, alcune dille quali sono cosi vane che per l'onore delle scienze naturali è meglio porre in oblio. 


\section{PARTE SPECIALE}

\section{SULLA FACOLTA RIPLODUTTIVA DELL'UONO}

L'uomo appartiene al gruppo dei vivipari mammiferi, ciò̀ l'orulo umano viene fecondato nell' interno del corpo della madre, dove si sviluppa in un ricettacoln particolare detto ulero, nel quale sboccano le trombe faloppiane che rappresentano i dotti escretori della glandula femminile (ovaia). Avvenuta la fecondazione l'uovo umano, come quello dei mammiferi, si melte in intimo rapporto coll'utero per lo scambio del materiale necessario al suo sviluppo, il quale rapporto subisce delle fasi di maggiore o minore intensità nelle diverse epoche della gestazione, fino a clie viene troncato col parto.

All'epoca della nascita gli organi germinativi, come tutto l'apparecchio genitale dell'uomo e della donna, sono poco sviluppati e rimangono per i primi anni della vita in uno stato di apparente inerzia, talchè anche il loro incremento iu volume non è proporzionale a quello degli altri organi. Ad una certa epoca ( piu presto d' ordinario nei climi caldi che nei freddi, nella donna che nell'uomo, nelle città clıe in r'ampagna) avendo gli altri organi e specialmente gli apparecchi della digestione e circolazione acquistato un lorte sviluppo, quasi clic la quantiti lel sangue e la lorza impellente del cuore fossero già maggiori del bisogno per la uutrizione degli altri organi, si verifica rapidamente uno stato iperemico nell'apparecelio genitale d'ambo i sessi, che ha per effetto un rapido sviluppo delle glandule germinali e di tutto l'apparecchio genitale.

Nelle prime si sviluppano o le cellule lecondabili (ovuli) o le cellule fecondatrici (corpuscoli spermalici), mentre l'appareceliso genitale si prepara alle.funzioni accessorie quali sono la copula e la gestazione.

Insieme a yuesti cambiamenti nell' apparecchio genitale 
se ne veriticano altri organici e psichici. Alcuni sono comuni ai due sessi come sarebbero: la comparsa dei peli al pube c l'istinto al sesso; altri sono speciali e tali da rendere anche con segni esterni più evidente la differenza dei due scssi. Cosi nell'uomo la comparsa della barba, l'abbassamento di voce, la risolutezza e l' ardirc consoni allo sviluppo delle forze fisiche; nella donna la comparsa dei mestrui, lo sviluppo delle mammelle e del pannicolo adiposo sollocutaneo, che impartisce a tutto il corpo ed alle singole parti forme tondeggianti; in fine una certa ritrosía timidezza, che rivelano l'occulto istinto al sesso appunto perchè si manifestano sol quando la donzella si trova coll'uomo.

Come rare anomalie s'incontrano talvolta dei giovani di forme muliebri e che sono maschi unicamente per l'apparecchio genitale, mentre sia per le forme generali, come per la delicatezza c timidezza dovrebbero ascriversi al sesso debole; cosi pure vi hanno delle donne, che diconsi viragini, perchè all'aspelto virile uniscono la risolutezza e l'ardire proprii del sesso forte.

Prima di passare all'esame della funzione riprociutliva gioverà fare una breve digressione anatomico-fisiologica sull'apparccchio genitale d'ambo i sessi nell'età adulta.

L'apparecchio genitale tanto dell' uomo che della donna consta d'un complesso di organi, i quali per la loro forma e funzione speciale permetlono una distinzione di tutto l'apparecchio in tre sezioni, cioè:

1. ${ }^{2}$ Sezione - organi o glandule germinali ; testicoli nell'uomo, ovaie nella donna.

2. $\quad i d$. - organi conduttori c conservatori dei prodotti degli organi germinali; deferenti, vescichette spermatiche nell' uomo ; tube, utero nella donna.

3." id. - organi della copula : pene nell'uomo, vagina nella donna. Questi organi comunicano coi germinali mediante gli organi conduttori. 
La terza sezione deli' ilpparecelio genilate d'anbo i sessi, ma piì specialmente nel maschio, per una certa eco-nomia dell' organismo trovasi in stretto rapporto anche coll'apparecchio urinifero, del quale non occorre qui far parola.

Negli organi conduttori e conservatori, che conformemente al loro ufficio consistono di cauali e serbato i e ricevono gli sbocchi di molte glandule esisteuti nello spessore delle loro pareti oppure al di fuori di queste ma che le attraversano coi loro dotti escretori, avvengono anche dei mutamenti fisico-chimici nei prodotti degli organi germinali. Queste glandule accessoric sarebbero a modo di esempio le prostatiche, le acinose dei derefenti e delle vescicole seminali nell'uomo, le uterine nella donna ec.

APPARECGHO GENITALE MASGHLE

I testicoli sono gli organi piu importanti dell' apparecchio genitale maschile (1); considerati anatomicamente e fisiologicamente hanno il signilicuto di semplici glandule escretrici, in quanto clre il loro prodotlo destinato unicamente alla ripro-

(1) La forma piủ comune della glandula germinale maschile negli animali è la tubulare; i tubulini ora sempliei, ora ramificati ed anastomizzanti fra loro sono d'ordinario tortuosi ed aggomitolati sopra di se slessi; terminano a fondo cieco o direttamente o dopo d'essersi ramificati ed anastomizzati. Sebhene la forma tipiea di questa glandula sia la tubulare, pure anche negli animali vertebrati ed a sesso distinto noi troviamo dei testieoli rappresentati da elementi vescieolari peduncolati e quindi costruiti sccondo il tipo delle glandule acinose od a grappolo, come si osservi uella Salamandra maculata, nolla Coecilia annulata, ed in molti pesei eartilaginei. In alcuni invertebrati crmafroditi, eome nella Rhodope, l'apparecchio genitale è rappresentato da una glandula a grappolo eon aciui distiuti, de' quali grli uni sono maschili, grli altri femminili; nelle lmmacle ed altri animali allini il folliculo del seme ce circondato da un' introflessione del sacco che contiene le nova. 
duzione della specie non ha aleun officio nell'economia animale dell'individuo.

Una borsa cutanea, scroto, situata sotto la radice del pene, conticne queste due glandule separate da un sepimento verticale, sello, di fitto tessuto connettivo; un solco poco profondo od una linea prominente, che ha l' aspetto di cicatrice, e clie si continua posteriormente nel rafe perineale ed all' innanzi nel rafe del pene, segna la divisione fetale dello scroto in due metà laterali; in corrispondenza di questa linea si stacca all'interno il setto di divisione.

Fra gli altri caralteri anatomici della pelle dello scroto merita d'essere ricordata l'assenza del pannicolo adiposo, il quale invece è sostifuilo da un robusto strato di fibro-cellule muscolari lisce (unica darlos), dal che ne viene la facilità con cui si corruga lo scroto per l'azione di stimoli diretti od iıdiretti, per esempio pel freddo. In questa condizione la pelle dello scroto presenta coll maggiore evidenza che quella di altre parti del corpo il fenomeno della pelle anserina, dipendente dalla posizione verticale che prendono i follicoli dei peli e le glandule sebacee, che ivi sono piuttosto sviluppate per numero e dimensione.

La cavità dello scroto può essere considerata come un'appendice dell'escavazione inferiore della pelvi, colla quale trovasi ini diretta jeomunicazione mercè $\mathrm{i}$ due canali inguinali, pei quali passarono i testicoli prima della nascita per discendere dalla carità addominale, ove si formarono, in quella dello scroto. E pertauto al di sotto della tunica dartos , fra gli altri involucri d'ogni testicolo noi dobbiamo trovare un' extroflessione del peritoneo, lunica vaginalis propria, che il testicolo ha incontrato e con se portato nello scroto nell'atto della discesa formandone un sacco peduncolato. Il peduncolo d' ordinario si oblitera, ma talfiata rimane pervio, come succede per regola generale nei mammiferi domestici. Sulla faccia esterna della vaginale propria vedesi uno strato di fibre muscolari lisce estriate, cremastere, il quale colle suc contrazioni può escrcitare una pressioue sulla glandula $c$, stante la sua 
inserzione al legamento del loupart, puó innalzarla verso la cavilit del bacino. (1)

La robusta membrana albuginea che involge direttamente il teslicolo è formata di fitto tessuto connettivo ed clastico; questa membrana manda verso l'interno dell'urgano moltissimi processi laminari, selli, e per tal modo la sostanza vera della glandula rimane divisa in tanti lobuli piriformi o conici colle basi rivolte verso la periferia e convergenti cogli apici verso quell'inspessimento dell'albuginea alla parte superiore posteriore del testicolo, conosciuto in anatomia solto il none di corpo d'Ilighmoro: questo tion è altro che un robusto processo dell'albuginea ove s'inseriscono i setti minori.

Gli elementi veramenle glandulari che formano i lobuli, di cui se ne contuluo più di cento in ogni testicolo, sono i canalelli escrelori o tubuli seminiferi, de' quali ogni lobulo ne contiene da tre in cinque. Questi tubulini sono lunglissimi e perciò avrolti più e più volte sopra se stessi a gomitolo; nel loro decorso torluoso si ramificano e terminano a fondo cieco oppure ad anse, per cni ritornano su di se stessi per sboccare all' apice del cono insieme agli altri in un canale maggiore e rettilineo. Da ogni lobulo esce pertanto un tubulino rello; dall'insieme di tutti questi \{ubulini che si anastomizzano fra loro nel corpo d'Higlımoro viene fórınata la cosí delta rele vascolare dell'Ialler, dalla quale poi escono da nove in diciasselte vasi efferenli. Questi escono dapprima rettiliuei, di poi si fanno tortuosi e sboccano finalmente in un callale a pareti spesse clie dicesi dollo deferente. I vasi efferenti sebbene sieno all' esterno del testicolo sono però ancora arvolti dall'albuginea, che manda dei processi fra un vaso efferente e l'altro e si continua in basso fino al principio del dotto deferente. Per lal modo si forma un corpo piriforme oblungo al margine posteriore del testicolo che si chiama epididimo.

(1) Alcuni indiridni hanno il canale inguinale cosi ampio cel il cremasterc talmente sviluppato o dipendente dallat volonta, che ponno ritirare a piacer lorn un testicolo o tulli e due nella cavitid dell'aldome facendoli eosl scomparire dallo seroto. 
I lobuli dei tubuli seminiferi sono circondati dai setti dell'albuginea, i quali alla loro volta mandano delle trabecole ucll’ interno dei gomiloli e con queste vi penetrano i vasi sanguigni che circondano colle loro reti capillari i tubulini. La parete dei tubuliui è spessa relativamente al loro lume; essa è formata di tessuto unitivo più o meno fibrillare all' esterno, omogenea verso il lume ove trovasi tapezzata da uno strato di cellúle epiteliali poligonali conteneuti dei granuli di materia grassa, dalla cui presenza dipende il colore giallognolo dei lobuli del testicolo nell'adulto.

I tubuli relti lianno la medesima struttura dei canalicoli, hanno però pareti più robuste e sono tapezzali internamente da epitelio cilindrico. Quando i tubuli retti si fanno tortuosi per formare i coni vascolosi, a ridosso della parete di conuettivo si trova un doppio strato di fibre muscolari, nell'uno longitudinali nell'altro circolari, e l'epitelio ciłindrico interno è munito di soltilissime ciglia vibratili.

Questi due fatti anatomici, cioè la presenza di fibre contrattili nelle pareti e di epitelio vibratile interno, che costituiscono due forze per tenere in moto e spingere innanzi il liquido contennto nei tubuli, ci indicano che siamo giunti agli organi della seconda sezione, cioè agli organi conduttori e conservatori del prodotto della glandula germinale, fra cui meritano essere distinti il dotto deferente e le vescicole spermatiché.

Il primo che incomincia alla coda dell' epididimo è un canale assai lungo, sottile, a pareti robuste e perciò con un lume assai stretto. Lo spessore delle sue pareti dipende specialmente dalla presenza di più strati muscolari che circondano il connettivo interno, il quale lıa già i caratteri di membrana mucosa, che d'ordinario è pallida e presenta delle pliche longitudinali e delle depressioni o fossette. In principio il dotto si vede tapezzato da epitelio vibratile, più imnanzi ri ha epitelio pavimentoso. Come si sa dill' anatomia il dolto deferente insieme ai vasi sanguigni, linfatici e nervi rimonta il canale iuguinale, entra nella cavifi addominale ore si porta alla pa- 
rete láterale posteriore della vescica urinaria e piegandosi in basso si dirige verso la linea mediana convergendo con quello dell'altro lato; finalmente quando sono giuuli al margiuc postcriore della prostata penetrano fraquesta e la parete dell'uretra per sboccare separati con un'apertura a becco di clarinetto su quell' emincuza della mucosa dell' uretra detta caput gallinaceum. Il tratto del deferente circondato dalla sostanza della prostata dicesi dollo ejaculalore.

Poco indietro del margine posteriore della prostata si vede staccare ad angolo acuto da ogni dotto deferente un' appendice tubulare, la quale si dirige all'indietro e si ramifica; lanto il tubulo primitivo come le sue ramificazioni presentano frequentissime varicosita piegate e divise da strozzamenti el angoli, di modo che formano nel loro assicme un corpo circonvoluto claviforme, vescicola spermalica lenuto assieme da tessuto cellulare misto a Gibre muscolari. Ed è in queste appendici cieche dei dotti escretori delle glandule germinali maschili che si raccoglie e conserva il prodotto delle stesse. Come nello spessore delle pareti del deferente, massime nella sua porzione prossima alla prostata, cosi auche nello spessore delle pareti delle vescicole spermatiche si trovano numerose glandulette acinose semplici, che coi loro dotti sboccano nel lume dei canali.

La struttura delle pareti delle vescicole seminali non è gran fatto diversa da quella del deferente; in generale sembrano pii esili per poco sviluppo degli strati muscolari. L'epitelio delle vescicole è parimenti pavimentoso poligonale.

Agli organi condultori e conservatori segue quello della copula, cioc̀ il pene o membro virile, il quale è un asta cilimlroide sporgente alla superficie del corpo e percorsa da nII canale, uretra, clie riceve posteriormente o nell'escavazione del bacino i due dolli ejaculatori esi apre allestremila libcra. Questo canale ì comune all' apparecchio urinilero e gocuilale, e percio serve all' emissione dell' urina ed all'ejarulizione dello sperma. Nella prima funzione è piutloslo passivo, dappoiche l'urina lo percorte e ne esce per forza 
muscolare delle pareti della vescica e della cavita addominale; c pertauto per questo ufficio l'uretra virile potrebbe aprirsi alla superficic del corpo come nella douna. La sporgenza di questo canale oltre la superficie del corpo e la particolare struttura anatomica di quel tessuto interposto fra il tubo mucoso (uretra nello stretto senso) e la pelle che involge tutto l'organo all'esterno, sono conformi alla sua funzione precipua cioè la copula, per la quale il pene deve mutare lirezione, volume, consistenza, ed infine deve essere munito d'un apparecchio opportuno, a spingere il liquido fecondatore nei genitali muliebri in quel modo particolare d'uscita a getto che dicesi ejaculazione, appunto perchè succede con certa rapidità e forza.

I nutamenti di direzione, volume e consistenza sono dovuti alla presenza dei due corpi cavernosi proprii del pene e del corpo cavernoso dell' uretra, il quale trovasi collocato nel solco inferiore fra i due corpi cavernosi proprii del pene oltre i quali fir sporgenza col rigonfiamento auteriore o glande. La distinzione del tessuto carernoso in tre corpi è puramente esterna, in fatli però essi formano un solo organo involgente il tubo mucoso uretrale, dappoichè le guaine involgenti i singoli corpi nei punti di reciproco contatto presentano molte aperture di comunicazione, sicchè dalle lacune di un corpo si passa in quelle dell'altro.

Ogni corpo cavernoso è circondato all'esterno da una robusta guaina fibrosa di tessuto unitivo ed elastico, la quale manda all'interno tanti sepimenti e trabecole che variamente s'intrecciano fra loro lasciando delle lacune di diversa capacilà, le quali contengono delle vene che aderiscono colla loro adventizia alle pareti formate dalle trabecole e dai setti. Cosi le lacuue rappresentano tanti seni venosi tapezzati intermamente, come è naturale, dall'epitelio proprio delle vene.

Le trabecole e le pareti stesse di queste vene sono formate da tessuto unitiro, elastico e da fibre unuscolari lisce. Nello spessore delle pareti delle lacume decorrono le arteric, alcune delle quali si risolvono nella rete capillare erl altre sboccano 
direttamente nelle vene. Un' altra caratteristica anatomica vuol' essere qui ricordata ed c̀ la prescriza delle cosi dette arecric elicine del Müller nei corpi cavernosi del pene presso al. La sua radice. Queste sono rappresentale da brevi rami arte. riosi dai quali si staccano tanti piccoli ramoscelli che forma110 dei pennelli, i quali dopo un decorso assai flessuoso e contorto si ramificano in rami naturalmente più piccoli ed avvolti a spirale penetranti nelle trabecole.

Da tutti questi fatli anatomici si rileva come il pene sia un organo cminentemente eretlile, verificandosi in esso tutte le condizioni favorevoli perchè possa subirc rapidi e considerevoli mutamenti di volume e consistenza col variare della quantità del sangue che vi afluisce e di quclla che effluiscc. Un maggiore afllusso, un impedito rillusso od ambedue queste condizioni simultanee produrranno l'aumento di volume e l'indurimento, mentre l'afflosciamento sarà l'effetto di condizioni opposte. (1)

Il decorso flessuoso spirale delle arterie elicine permette che questi vasi durante l' erezione si allunghino svolgendosi senza lacerarsi. Anche i tegumenti che ricoprono il pene sono molto lassi e facilmente estensibili; il tubo mucoso uretrale può parimente allungarsi e spiegarsi.

L' erezione o mutamento di direzione dipende dall' essere i corpi cavernosi proprii del pene brevi, robusti e fissati saldamente colle rispettive radici alle ossa del bacino, mentre il corpo cavernoso dell' uretra c̀ gracile, assai lungo e quasi libero.

Per intendere il modo con cui si possono verificare i mutamenti di pressione del sangue nei corpi cavernosi, non che il meccanismo dell'ejaculazione è d'uopo ricordare alcuni particolari anatomici dell'uretra e dei muscoli annessi all'apparecchio della copula.

(1) Inicttapdo una massa (qualunqque nell' arteria pudenda comune dei cadaveri si otliene l'crezione completa del pene, cio che prova come questa difenda dalla raccolla di sangrac uci corpi careruosi. 
La parte dell' uretra che trovasi nell' escavazione del baci110 si caratterizza per lo sbocco dei due dotli ejaculatori sull' eminenza detta capul gallinaginis, clıe è formata da una ripiegatura della mucosa dell' uretra stessa poco innanzi del collo della vescica urinaria da cui l' uretra si origina. In questo tratto dell' uretra sboccano i numerosi dottolini di quelle glandulette acinose, che in numero di circa quaranta circondano l'uretra e clie per mezzo di forle tessuto interstiziale di conueitiro e fibre-muscolari liscic costituiscono il corpo della prostatı. Questa è pertanto un anello glandulare e contrattile clic abiraccia per un certo tratto l'uretra. Alla parte prostalica dell'ure tra segue la parte membranacea, che è rappresentafa unicamente dal tubo mucoso uretrale che attraversa il m. diaframma pelvico. Appena oltrepassato questo muscolo il tubo mucoso aretrale viene circondato dal suo corpo cavernoso, che in principio presenta un rigonfiamento, bulbo, simile a quello che sta all' estremità libera o glande. In corrispondenza delia parte bulbosa sboceano nell' uretra i dotli di Jue altre glandule acinose dette glandule del Cowper.

Come parte componente essenziale dell' apparato della copula si trovano nell' nomo due muscoli a Gibre striate, uno impari e l'altro pari, di cui ciascuno appartienc al corpo cavernoso del lato corrispondente.

Il muscolo impari o bulbo-cavernoso è rappresentato da uno strato muscolare che copre il bulbo dell' uretra alla sua faccia inferiore. Consta di una meta destra e di una siuistra, tra cui si trova una striscia tendinea longitudinale a eui s'inseriscono lateralmente le fibre muscolari, in modo cle ne risulta un muscolo bipennato. I fasci muscolari d'ambo i lati decorrono obliquamente all' innanzi ed all' esterno ; la maggior parte di essi formano degli anelli obliqui intorno al bulbo dell' uretra poichè vanno ad inserirsi ad un' altra striscia tendinea sulla parte superiore del bulbo ; pochi fascetti muscolari anteriori passano in una lanima tendinea, la quale si fonde ron cuclla dell' altro lato in modo che l' ansa rhe ne risulta 
abhraccia la parte posteriore del tronco del pene in vicinanza dei vasi maggiori dello stesso. L'azione di questo muscolo si \& di spingere il sangue dal bulbo verso il glande e di comprimere il bulbo dell' uretra per espellerne il contenuto ; perciò si cliama anclie muscolo acceleralore dell' urina ed ejaculatore del seme.

Questo stesso muscolo colla sua ansa tendinea supcriore può impedire anclıc l' uscita del sangue per la vena dorsale comprimendola nell'alto che si contrac, ciò che appunto verificasi durante l'cjaculazione, momento in cui la turgescenza del pene raggiunge il massimo grado.

Il muscolo ischio-cavernoso (pari) incomincia presso la tuberosità ischiatica ed alla cresta del penc del bacino all' interno della radice corrispondente del corpo cavernoso del pene ; si avvolge quindi a spirale intorno alla radice passando all'esterno della stessa e termina in un tendinc piatto e largo, il qualc s'inscrisce alla guaina fibrosa della radice dove questa s'incontra ad angolo con quclla dell' altro lato. Il decorso spirale dei fasci muscolari di questo muscolo fa si che esso rappresenta un robusto compressore del corpo cavernoso del suo lato e nello stesso tempo lo innalza adducendolo verso l'addome. Per questa scconda funzione venne chiamato muscolo erettore del penc.

Le arterie dell' organo della copula sono per la massima parte diramazioni dell'arteria pudenda comune, il cui decorso, diramazione e distribuzione ci sono noti dall'anatomia descrittiva; lo stesso si dica per le vene, che sboccano in parte nella vena pudenda comune ed in parte per mezzo del plesso prostatico nella vena ipogastrica, per cui la vena pudenda comune od interna è proporzionalamente piccola. Infine notiamo come nel pene piu che in altri organi del corpo i rapporti topografici delle vene sono tali per cui facilmente vengono ad essere compressc. (1)

(i) Il plesso venoso prostatico in cui si versa non solo il sangue della vena 
I nervi dell'apparecchio genitale dell' nomo sono rami de! sistema nervoso cerebro-spinale (nervi erigenti e pudendo) ed iu parte del simpatico. I primi contengono tubulini centripeti e centrifughi. Dai centripeti dipende la sensibilità di tutto l'apparecchio, che negli organi esterni raggiunge il grado massimo della sensibilità taltile cle c̀ di una particolare natura (volultì sessuale), mentre negli organi interni si fa sempre più ottusa non essendo suscettibile che delle sole sensazioni dolorose.

Per le esperienze di Ecklard si conosce che irritando nei cani alcuni ramoscelli nervosi provvenienti dalla porzione sacrale del plesso ischiatico si otliene l’ erezione del pene, la quale iucomincia al bulbo dell'uretra e procede innanzi verso il ghiaude. Durante l' eccitamento di questi nervi si verifica un forte afllusso di sangue arterioso al pene. Questi nervi venucro perciò chiamati erigenti od erellori. Il nervo centripeto pei movimenti riflessi sarebbe invece il nervo pudendo comunc; la sua recisione ì seguita sccondo Löven da dilatazione dell'arteria dorsale del pene ed aumentata pulsazione della stessa.

In proporzione del grado di sensibilità diqueste parti le troviamo suscettibili di provocare eccitamenti psichici o riflessi semplici o fenomeni automatici. Con ciò non si vuol dire che le attività funzionali sessuali sieno sempre l'effetto di reazioni per eccitamenti dei nervi centripeti, dappoichè noi sappiamo come l' erezione c l' ejaculazione possono essere direttamente provocate da attività psichiche. Questo rapporto degli organi sessuali c specialmente di quelli della copula col comune sensorio per la via del midollo spinale viene confermato dall impotenza all crezione ed ejaculazione nei casi di malaltie del midollo spinale, come pure dall'erezione ed eiaculazione per

dorsale ma anche una gran parte di quello della profonda rappresenta cone un argine regolatore della quantila del sangue che effluisce dal penr, per rui non potendo si facilmente aumentarne l'eflusso un leggier aumento mell'alllusso produce l' erezione. 
eccilamento dello stesso anche nelle regioni superiori (strangolamento).

I nervi di moto per quanto si è già detto saramo in parte dipendenti dalla volontà ; però la maggior parte dei movinıenti che ivi si verificano sono d'origine riflessa, cioè vengono provocati da eccitamenti avvertiti dalla coscienzil, oppure di nalura automatica, perchè percorrendo i nervi centripeti nou raggiungono il comune sensorio. Infatti i muscoli ischio-caveruosi e bulbo-cavernoso si muovono automaticamente, per forza di volonta e per riflesso, mentre gli altri muscoli si. contraggono od automaticamente o per riflesso o per movi. mento associato.

I rami del gran simpatico servono specialmente ai fenomeni trofici ; cosi al testicolo ed ai dotti deferenti insicme ai vasi sanguigni vi arrivano, dei rami del gran simpatico; come quasi tutti gli altri rami del sistema ganglionare anche i nervi dei deferenti contengono evidentemente fibre centrifughe e centripete; e perciò la loro irritazione riesce dolorosa ed ì seguita da contrazioni vermicolari dei deferenti. I tubulini di moto contenuti in questi nervi derivano, secondo Budge, dalle radici del $4^{\circ}$ e $5^{0}$ nervo lombare, e perciò avrebbero origine dal centro genito-spinale, che secondo lo stesso Autore trovasi nella sostanza grigia della midolla spinale all' altezzi della $4^{a}$ vertebra lombare.

Eckhard avrebbe osservato dei ganglietti lí dove i rami nervosi erigenti si distribuiscono nei corpi cavernosi.

\section{APPARLCCHO GLNTALE DELLA DONNA}

Le ovaic sono due corpi amigdaliformi situati nella caviti della pelvi ed attaccati all' atero mercè un lungo corilone fibroso. Queste glandule sorro involte da rua ripiogatura del peritonco (legamento largo od ala di pipistrello) e percio il tessuto glandulare ne ì tullo circondato.

La sostanza propria dell ovaia dicesi con unene gencrico 
stroma; consta di un fitto tessuto cellulare in cui si distribuiscono i vasi sanguigni, i linfatici ed i nervi. Il tessuto connettivo alla superficie della glandula è molto denso, povero di vasi sauguigni e presenta una superficie piuttosto levigata, per cui vienc considerato come uno strato o membrana iuvolgente che si distingue col nome di albuginea (1).

Siccome il peritoneo involge tutta la glandula, il prodotto della stessa, cioè le cellule germinali od ovuli, devono romperlo per uscirne. Allora vengono raccolti dall' apertura imbutiforme erettile ed a bordo frangiato della tromba faloppiana la quale d'ordinario è attaccata all'ovaja con uno o più fimbrie.

Spiegelberg e Letzerich trovarono nell'ovaia del feto uma110, come Pflüger pel primo descrisse nello stroma dell' avaia di altri mammiferi, tanti tubulini di diversa lunghezza e diametro, clie dalla periferia della glandula convergevano verso il centro della stessa. Questi tubulini ora semplici ed ora divisi dicotomicamente sono spesso contorti ed avviluppati. Secondo queste recenti osservazioni l'ovaia dell'uomo e degli altri mammiferi sarebbe pertanto una glandula tubulare analoga al testicolo dei mammiferi ed all' ovaia di molti animali invertebrati (2). Nelle ovaie di bambine neonate clie esaminai col mio allievo Renzone lio osservate tante introflessioni tubulari dall' aspetto di fiale strette e lunghe col fondo cieco rivolto verso l'interno e coll'apertura piuttosto ristretta alla superficie, e ci siamo assicurati che il peritoneo vi pren-

(1) Nei feti inveee la superfieie dell' ovaia è spugnosa e hucherellata per lc introflessioni recenti del peritoneo.

(2) Negli animali vertebrati ed invertebrati si trova assai di frequente l'ovaia costruita sceondo il tipo delle glandule aeinose od a grappolo; io sono però d'avviso che queste apparenze sieno pintlosto eonseculive e dipendenti da effetto meceanico, eioè dallo sviluppo degli ornli nei tululini primitivi, i quali, come è naturale, vengono a distendersi e formare dei rigonfiamenti. Le mie ricerche in proposito mi conviusero che in molti animali l'ovaia assume l'aspetto di mua glandula acinosa sultanto quando le uova s'ingrossano e si preparano ad abbandomarla, mentre nei primi lempi di vita corrisponde ad ma glandula tubulare. 
de parte giaches allontanando le cellule si vedeva la parele lei tubulini continuarsi all'esterno nell'iuvolucro della glandula.

Resta pertanto ben constatato che i tubulini descrilli hatıno una parete propria e non sono unicamente spazi o canali pieni di cellule scavali nello stroma.

Gli organi condultori e conservalori dell' apparecehio geuitale della doma sono rappresenlali dalle trombe faloppiane c dall' ulero. Bisogna però nolare che quesli organi nonk servono soltanto come i corrispondení organi maschili a conservare e condure il prodollo delle glandule rispellive, ma ben anche sono chiamali a ricevere e conservare il licuido fecondatore.

Come le ovaic corrispondono ai testicoli cosi le trombe faloppiane sono gli analoglii dei dolli deferenti. E per vero anche queste sono rappresentate da un lungo e sottile cordone, percorso da un canale angustissimo, le di cui pareli conslano di tre tuniche, esterna peritoneale, media muscoscolare liscia, interna mucosa la quale è poi tapezzata da un epitelio cilindrico vibratile; ¿ quindi anche nelle tube si trovano analoghe forze locomotrici del contenuto, cioè contrazioni muscolari e vibrazioni di ciglia. La differenza esscuziale fra questi canali e gli analoghi del maschio sta iu ciò che i tubulini dell ovaia non sboccano dirctlamente nell' ovidutto, il quale invece all' estremità ovarica perfora il peritonco e si allarga a mó di un imbuto a bordo frangiato e libero nella cavità addominale. Le trombe faloppiane con un decorso leggermente flessuoso e discendeute dall' esterno verso la linea mediana del corpo, raggiungono il fondo dell' utero ed altraversandone le robuste pareti sboccano nella sua cavita.

L'utero è ull sacco muscolare piriforme capovolto, che rol suo apice smuzzato ed aperto sporge in un canale membranaceo più o ineno cilindrico dellu vebsina, la quale si adalta in allo alla superticie esterata del collo dell'utero ed 
in basso si apre colla vulva allo esterno. Tutte queste parti comunicanti tra loro solıo lapezzate internamente da una membrana mucosa ricca di vasi e nervi, sulla quale versano i loro umori moltissimeglandule, alcure delle quali si trovalıo nello spessore della inucosa stessa (glandule utriculari uterine) ed altre (acinose o mucose propriamente dette) si trovano all' esterno della mucosa e l' attraversano coi loro dotti ( vaginali).

L'umore alcalino segregato da queste glandule versandosi sulla superficic della mucosa serve a mantenerla lubrica, nonchè a conscrvare $\mathrm{i}$ corpuscoli spermatici ed a facilitarne il cammino all'incontro delle uova.

La mucosa uterina è tapezzata da un epitelio vibratilc, mentre la mucosa vaginale possicde un epitelio pavimentoso a più strati ; le pareti muscolari dell'utero sono assai robustc; le suc fibre sono lisce ed hanno un decorso assai intricato, soltanto all' orificio interno del collo sono disposte regolarmente a mo' di un cercine, per cui si considerano come un muscolo speciale che dicesi sfintere dell'ulero. La cavità di questo sacco muscolare è triangolare coi lati retti; durante la gestazione si dilata c diventa ovoidale, ma le pareti non si assoltigliano, anzi diventano più robuste per sviluppo di nuovo tessuto muscolare. La mucosa della vagina presenta molte ripicgature che rendono possibile la sua dilatazione per la copula c più ancora per il parto; come per la presenza di fibre muscolari circolari all' esterno della mucosa può stringersi e diminuire il suo lume interno.

L'utero c̀ mantenuto nella sua posizione dai legamenti larghi e dai legamenti rotondi i quali sono formati da tessuto cellulare c contengono fibre muscolari lisce.

Non è difficile di riconosćce l'analogia fra i pudendi csterni della donna c quelli dell' uomo. Come vedremo piu tardi la differenza nelle apparenze puó cousiderarsi come l'effetto di un arresto di sviluppo in alcunc parti egualmente abbozzate nei feti d'ambo i sessi. Nello bimbine le piegghe 
cutance laterali all ostio uro-genitale non arrivano a saldarsi fra loro nel!a linea mediana per formare lo siroto e rimangono divise e divaricate (grandi labbra); i corpi cavernosi proprii del peue rimangono in uno stato rudimentale e perciò nascosti nell' angolo superiore delle grandi labbra (clitoride). Da ciò ne viene íl diversó sviluppo e configurazione del tessuto cavernoso dell' uretra non che del muscolo bulbo-cavernoso. Questo si foggia a muscolo sfintere o costrittore esterno della vagina, quello si estende per formare un tessuto crettile interposto agli strati muscolari dalla vagina e con due processi latcrali si porta all' estremità del clitoride per formarvi un ingrossamento spugnoso che corrisponde al ghiande. Riguardo ai vasi sanguigni dei genitali muliebri fa d' uopo ricordare i seguenti fatti :

$10^{0}$ Clie l'arteria spermatica, ramo dell'aorta, attraversa il legamento largo dell'utero e si anastomizza coll'arteria uterina del lato corrispondente indi si porta all'ovaia.

$20^{\circ}$ cle alle trombe faloppiane vanno dei piccoli rami delle duc arteric suddette, i quali decorrono fra le lamine dei lcgamenti larghi.

$3 .^{\circ}$ che la vagina riceve rami dall'arteria cmorroidaria media, dalla vescicalc e dall'uterina.

$4 .^{0}$ che i pudendi esterni ricevono dall'ipogastrica l'arteria pudenda comune, la quale si comporta presso a poco come nel maschio.

$5 .^{\circ}$ clic le vene interne cioè: l'uterina, la spermatica, la vaginale formano dei plessi che spesso si anastomizzano fra loro.

$6 .^{\circ}$ che le vene esterne si comportano presso a poco come le corrispondenti nell'uomo.

I nervi dell' apparecchio genitale muliebre sono come nell'uomo rappresentati da rami appartenenti al sistema nervoso cercbro-spinale ed al sistema ganglionare o del gran simpatico. Qnesti ultimi decorrono in forma di plessi colle arteric (spermatica, uterina, pudenda) c vanno a distribuirsi negli organi corrispondenti. 
Il plesso spermatico nasce dal plesso renale, gli altri dal plesso ipogastrico inferiore. I rami dell'assc cerelıro-spinale vengono dal pless o pudendo ed in parte vanno all'utero ed alla vagina, in parte ai pudendi esterni.

1 tubulini nervosi molori dell'utero o vanno pel midollo spinale direttamente al cervello, oppure sono in rapporto con questo mediante centri esistenti nel midollo spinale. Infatti si ponno provocare contrazioni dell' utero irritando il midollo spinale, il midollo allungato, il cervelletto, il ponte ed altre parti del cervello; le contrazioni variano per modo, velocità ed energia a seconda che l' eccitamento vien fatto nell' uno o nell'altro de'punti suindicati dell'asse cerebro-spinale; cosi' la regione lombare del midollo spinale, per le ricerche comparalive di Obernicr, sarebbe la più influente sui movimenti dell' utero.

Siccome l' utero degli animali continua ad eseguire delle contrazioni ritmiche per un certo tempo dopo che è stato tolto dal corpo, come succede dell' intestino, del cuore, etc. cosi si suppone che nello spessore delle sue pareli esistano degli ammassi ganglionari i quali rappresentano uno o più centri automatici. Secondo alcune esperienze di Kehrer questo centro aulomatico dovrebbe trovarsi al confine fra la vagina e l'utero, dappoichè questo continua le sue ritmiche contrazioni dopo il taglio dei nervi sacrali finchè non si stacca dalla vagina.

L'eccitamento dei nervi sacrali produce forti contrazioni dell'utero, mentre i nervi spermatici non esercitano alcuna influenza diretta sui movimenti dello stesso.

All'epoca della nascila e uci primi anni di vita i canaletti spermalici sono assai soltili e ripieni di tante cellule nucleate e trasparenti, molto simili alle cellule di secrezioue di altre glandule. Poco prima della puberti, la yuale, come abbiano veduto innanzi , nell' nomo si icrifica più tar- 
di che nella donna, i tubulini s' ingrandiscono e le cellnte in essi contente incominciano a differenziarsi in parietali e centrali; le prime mantengono i caralteri di cellule epiteliali; le seconde o centrali s'ingrossano mentre il loro protoplasma si fa granuloso ed opaco; c finalmente uell' interno del protoplasma si vedono formarsi dei nuclei vescicolari trasparenti da prima sferici poscia ovoidali. Non tutte le cellule centrali raggiungono l'istessa dimensione, e quindi è anche diverso il numero dei nuclei vescicolari in esse contenuti; nelle più piccole si osserva un solo nucleo, nelle più voluminose se ne trovano da dieci a venti. Dai nuclei vescicolari si sviluppano, secondo Kölliker , i corpuscoli spermatici. Ogni vescichetta allungandosi si differenzia in una porzione anteriore a contorni molti oscuri, ed in una porzione più piccola posteriore a contorni pallidi; queste due parti della vescichetta non solo differiscono nell' aspetto, ma si comportano diversamente coi reagenti chimici, così a mo' d' esempio la posteriore assorbe facilmente l'acqua, si gonfia e scoppia. Più tardi dal polo o segmento posteriore si vede sporgere un' appendice filiforme che si allunga assai a spese della parte pallida della vescicola. In fine la porzione anteriore assume la forma tipica che ha il corpo del filamento spermatico come si trova nello sperma ejaculato.

I filamenti spermatici sviluppati rimangono ancora per qualche tempo nella cellula in cui si originarono, e siccome il diametro delle cisti spermatofore è più corto dei filamenti, questi si trovano piegati in cerchio più o meno completo; nelle cellule che contengono molti filamenti si vedono questi disposti in cercli concentrici uniti ed allineati colle teste rivolte da un lato e colla coda dall' altro. La cellula infine si rompe 0 , secondo alcuni, vienc rotta dai filamenti clie incominciando a muoversi si distendono. $\Lambda$ ppena usciti si manlengono per un certo tempo uniti in manipoli cui si reggono alerire i lembi della cisti scoppiata.

Le cisti ed i filancuti (isolati od in lasci) vengono portati 
mnanzi nei tubulini dalla vis a tergo delle nuove cellule che sorgono e probabilmente anche da un fluido (essudato sanguigiro) che si raccoglie continuamente nei tubulini spermatici, passando per fillrazione o diffusione attraverso alle pareti dei vasi sanguigni che circondano i suddetti tubulini. A questo essudato si aggiunge anche il liquido contenuto nelle cisti spermatofure.

Queste forze spingono i filamenti spermatici dai canaletti contorti nei tubuli retti, da questi nella rete vascolare dell'Haller, nei vasi efferenti e finalmente nel deferente. Lc vibrazioni delle ciglia dell'epitelio vibratile che tapezza all' interno i vasi efferenti, i coni vascolari ed il principio del dotto deferente, e le contrazioni peristaltiche di quest' ultimo contribuiscono ad imprimere il movimento progressivo allo sperma.

Già nel dotto deferente deve aver luogo una leggiera diluzione per diffusione fra lo sperma contenuto nel lume del canale ed il sangue cho scorre nei vasi sanguigni della mucosa che tapezza internamente questo canale, e probabilmente anche con quello dei più esterni esistenti nello spessore della tunica muscolare o dei plessi che avvolgono il dotto. Siccome lo sperma è molto più denso del sangue è naturale che questo cederà al primo la sua parte acquea. L'ingrandimento della superficie di diffusione e secrezione che si verifica nelle vescichette seminali, le quali sono niente altro che appendici cieclse e ramificate del dotto deferente, favorisce sempre pii la diluzione (1) dello sperma dappoichè nell' interno delle vescichette si raccoglic contiuuamente un liquido trasparente, viscoso il quale si mesce allo sperma mano mano che vi arriva.

Lo sperma che si raccoglie dal testicolo e dal. dotto delerente è molto denso, bianchiccio, inodoro e neutro; soltanto colla Giltrazione o coll' analisi microchimica vi si puó ricono-

(1) Diluzione uel seuso che st aumenta il meustrue iu confrouto del hukero de'filimeuti. 
secre la presenza della parte fluida rle tiene in sospensione filamenti e le cellule; questi elementi sono in si grau numero che i filamenti sono impediti nei movimenti. $\Lambda$ ggiungendo però qualche goccia di una soluzione di zucchero od alcalina pinttosto concentrata (a preferenza fosfato o carbonato di sodi) si redono beu presto agitarsi e muoversi con una velocilia che venne calcolata di un' oncia parigina in dieci minuti (1).

1 filamenti spermatici dell'uomo hanno il corpo o testa della figura di una mandorla schiacciata; veduti di piotto sono pallidi ed a contorni poco distinti, mentre di profilo hanno contorni distintissimi c rifrangono assai la luce.

La lunghezza della testa è di . . 0,0016"

) larghezza . . . . . . 0,0008 - 0,0015

Lo spessore . . . . . . 0,0005 - 0,0008

La coda clic incomincia con un piccolo rigonfiamento sembra della lunghezza di $0,02^{\prime \prime \prime}$; 110 è però beu certo sc veramente coi nostri microscopii si possa vederne l'estremilà. Noll è ancora ben determinalo se il corpo sia solido o pure sia rappresentato da una vescichetta ripiena d'un liquido, come purc ¿̀ incerto il significato di certe macchictte assai trasparenti e lucide(vacuoli) che spesso si vedono al centro della testa, e che alcuni considerano come nuclei ed altri come infossamenti o spazii vacui. I corpuscoli spermatici constano di molta sostanza solida organica ed inorganica; infatli essi conservano le loro forme c dimensioni non solo dopo di essere stati essiccati, ma anche dopo la calcinazione, sempre che si facciano scceare e si riducano in cencre con certe cautele.

Lo sperma ejaculato per l'uretra presenta dei caralteri particolari per essersi mescolato con altri umori sia durante la

(1) La locomozione, come si disse altrove, è dovuta al filamento o coda; peró anche il corpo o testa secondo alcuni oscilla in virtủ delle sue proprie contrazioni per le quali, come i naturale, deve mulare anche di forma. Per altro io non ho mai potuto osservare questi mutanenti di forma ne' corpu-
scoli spermatici lreschi 
sua dimora nelle vescichette come nel suo cammino attraverso ai dotti ejaculatori ed al canale dell'uretra. Perciò può essere paragonato all'acqua d'un fiume la quale cambia dersità, colore ecc. mano mano che si avvicina alla foce, perchè riceve le acque d'altri torrenti e fiumi che sboccano o nel letto del fiume stesso o nei laghi ove si allargano e stagnano le suc acque.

Così allo sperma si unisce l'umore segregato dalle glandule delle pareti dei deferenti, delle vescichette, quello delle glandulc del Cowper, l'umore prostatico ecc. e perciò quando esce per l'uretra è bianeo-gialliccio, trasparente, filante, alcalino ed ha un odore suigeneris, clic alcuni paragonano a quello della farina di castagne ed altri a quello della segatura delle ossa o delle corna; questo sperma appena ejaculato è denso e gelatinoso, per cui i filamenti clıe tiene in sospensione sono pressochè immobili, dopo qualchı tempo si fa fluido forse per acqua igroscopica ass orbita (1).

La produzione degli elementi fecondanti nell'uomo è contiuua e non periodica come succede degli ovuli nella donna, e per ciò l'uomo dalla pubertà in avanti può scmpre avere delle polluzioni od ejaculazioni. L' uomo conserva pii a lungo che la donna il suo potere riproduttore, anzi nousi è potuto ben determinare a qual'elà l' uomo possa dirsi assolutamente impotente.

Nei mammiferi e negli altri animali (specialmente in quelli che vivono liberi) si osserva una periodicità nell' at-

(1) Si credeva d'aver assicurata la natura animale dei filamenti spermatici vedendoli arrestare istantaneamente ne'loro movimenti per l'aggiunta di tintura d'oppio, intcrpretando in buona fede l'azione rareotica di questa sostanza e non pensando ehe la tintura d'oppio conteneva anche aleool. Ma quando si tentò di ottenere l'arresto dei movimenti nei filamenti spermatici colla tintura acquosa si trovò che l'oppio per se stesso era innoeuo. L'aleool puro invece li arresta nei movimeuti perehè li altera chimieamente come l'etere, il cloroformio, il creosoto, l'acido tannico, gli olii eteriei. l'acido acetico, i sali dei metalli, gli acidi inorganici, tutte sostauze clic alterano i corpi albuminoidi e per l'ordinario li fauno coagulare. 
livita dell' apparecelio riprodultore non solo nelle femmin ne na anche nei inaschi, i quali all' epoca degli amori mutano spesso d'aspetto, di naturale, di costumi e facendosi l'ordinario arditi ed irrequieti sostengono tra loro delle fierissime lotte per disputarsi la lemmina; lotte che terminano in vantaggio della specic poichì soccombono i deboli e rimangono palroni del campo d'amore i più forti c robusti .

SVILUPPO DEGLT OVULT NELLE OVATE E LORO USCITA

Nei tubulini dellc ovaic formansi gli ovuli presso a poco come in altre glandule di secrezionc si formano le cellıle secretoric (moltiplicandosi anche per gemmazione o scissione) colla differenza pero che i singoli ovuli, i quali da principio si trovano disposti in seric continue nel tubulino, si isolano in seguito gli uni dagli altri incapsulandosi in scompartimenti o strozzamenti del tubulino e si circondano poi del tessuto o stroma dell' ovaia, in modo che si hanno delle cisti o follicoli chiusi contenenti un liquido e tapezzati da cellule, che in un punto sono assembrate in un cumulo attorno all'ovicino.

L'ovulo maturo dell'ovaia della donna, come quello degli altri mammifcri è pertanto contenuto in una cisti chiusa detta follicolo del Graaf, sporgente alla superficic dell'ovaia, le di cui pareli constano di più strati disposti dallo esterno all' interno nell'ordine seguente: $1^{0}$ peritonco, $2^{\circ}$ tessuto connnetlivo filto, $5^{\circ}$ tessuto conneltivo ricco di vasi, $4^{\circ}$ membrana omogenea soltile, $5^{\circ}$ strato di piccole cellule cpitcliali nuclcate e trasparenti ; a maturila perlelta del follicolo l'ovulo circondato dal stro cumulo di cellule occupa la parte della cisti che sporge alla superficic dell'ovaia. La cavità del follicolo come si disse è uccupata da un liquido limpido, giallognolo, alcalino ed albuminoso, il quale aumentando sempre più distende le pareti del follicolo, che finalmente scoppia laddove trova la minore resistenza, cioc alla superficic libera dellovaia e molto probabilmente in corrispondenza dell'antica apertura slell'introflessio- 
Ile peritoneale,come si vede chiaramente nella deiscenza dello stigma degli ovipari. E perciò lo scoppio del follicolo non deve cousiderarsi come una lacerazione ma piuttosto come il riaprimento d'un meato che s'cra obliterato.

$\Lambda$ pertosi il follicolo ne esce il liquido ed insieme a questo l' ovulo circondato dal cumulo delle cellule della membrana granulosa.

Quando si credeva che il follicolo del Graaf fosse l'ovulo, il cumulo di cellule che lo circondano si chiamava disco proligero, perchè si considerava come la parte essenziale dell'ovulo destinata a formare l' embrione. Oggidl, sebbenc da alcuni embriologi si usi ancora questa denominazione, noi la troviamo veramente inopportuna, ed accettiamo invece quella di disco ooforo appunto perchè circonda e porta il vero ovulo.

MENSTRUAZIONE

Nei climi tempcrati lo scoppio del primo follicolo e la caduta del primo ovulo si verifica nella donna fra il dodicesimo ed il sedicesimo anno. Da questo momento (pubertà) la donna diveuta fecondabile, ed a meno di condizioni morbose si mantiene talc fin verso i cinquant' anni, dappoichè lo scoppio d'uno o più follicoli si ripete una volta per ogni mese lunare.

La caduta dell' ovulo è accompagnata e forse preceduta da un flusso di sangue venoso, che esce per la vulva misto a variabili quantità di muco. Siccome questo fenomeno verificasi periodicamente ogni mese lunare, cosi fino da tempi antichissimi si appellò flusso menstruo o luna. I medici dell'antichità sapevano benissimo che la donna incominciava ad essere fecondabile colla prima menstruazione e clie cessava di esserlo coll' ultima; essi conoscevano pure che d'ordinario non comparivano i menstrui durante la gestazione e l' allattamento. Spiegavano percio il fenomeno ammettendo che periodicamente si verificasse un flusso di sangue all' apparec- 
chio genitale della doma per prepararlo alla gestazione e per servire al alimentare il nuovo essere nel caso di avvenuto coito fecondo: in caso opposto si scioglieva la pletora per trasudamento di sangue. Ora però si ritiene che il flusso menstruo corrisponda ad una vera emorragia, cioc̀ si verifichi per rottura di molti vasellini sanguigni della mucosa uterina, perchì il sangue meustruo contiene molti globuli rossi e non si sa facilmente spiegare la loro uscita attraverso alle pareti dei vasi, cioc̀ per trasudamento(1). Il sangue menstruo, forse perchè misto a diverse quantità di muco, differisce per molti caratteri dal sangue che esce da altri vasi ed organi ì di colore oscuro, coagula assai difficilmente ed è molto denso ed alcalescente.

Secondo una recente ed assai probabile teoria si spicga la maturanza e lo scoppio dei follicoli, non che il treno dei fenomeni che l'accompagnano in un modo abbastanza convincente.

I vasi sanguigni dell' utero, delle ovaic e delle tube, per le note condizioni anatomiche di struttura, forma, disposizione, decorso, modo di diramarsi ed anastomizzarsi , danno agli organi genitali interni della donna il caraltere di organi erettili, per cui possono con una certa rapidità aumentarc di volume per maggiore afllusso di sangue o per impedito efllusso dello stesso. Pertanto secondo questa teoria la maturanza dell' ovicino, la forte distensione del follicolo per la raccolta nello stesso di gran quantità di liquido, che finalmente ne produce lo scoppio, il turgore della mucosa uterina, l'abbarbicarsi all'ovaia delle frangic dell'apertura imbutiforme della tuba per ricevervi l' ovulo quando esce dal follicolo, il flusso sanguigno per rottura dei vasi c l'aumentata secrezione operata per le glandule mucose dei genitali interni, sarebbero preceduti o meglio provocati da una specie di erezione passiva di tutti gli organi genitali interni della dorna ; erezione

(1) Con tulto rii nessuno ha peró mai potuto vedere le lacerazioni dei vasi sanguigni. 
dipendente da impedito efllusso del sangue venoso per unit contrazione spasmodica o continua della muscolatura dell' utero e delle fibro-cellule muscolari che trovansi disposte alcune longitudinalmente ed altre iu direzione trasversaleobliqua nel mes-ovario, cioè fra le pagine del legamento largo.

Siccome per la contrazione vengono ad essere compresse a preferenza le vene e meno le arterie, stante la robustezza e l'elasticità delle pareti di quest'ultime, ne viene che continuando le arterie a portare la solita quantità di sangue ai genitali interni, mentre ne è impedito l'efflusso per le vene compresse, si verifica una pletora con rallentamento nel corso del sangue ed aumento di pressione laterale sulle pareti dei vasi.

A questa teoria di erezione passiva per compressione delle vene si vorrebbe oggidi contrapporre l' altra ammessa da Ecklard per spiegare i fenomeni d'erezione nel maschio, la quale potrebbe dirsi attiva, poichè dovrebbe verificarsi in seguito d'un aumento nell'afflusso di sangue arterioso per dilatazione delle arterie, le quali perderebbero il tono muscolare delle loro pareti per eccitamento di nervi arreslatori od antagonisti dei vaso-motori.

Comunque sia, abbiamo sempre una condizione cle farorisce la filtraziono copiosa di plasma sanguigno attraverso alle pareti dei vasi nello stroma dell'ovaia e nell' interno del follicolo che si distende e poi scoppia. Cosi pure per la pletora possono rompersi molti vasellini capillari uterini da cui ne esce il sallgue, che scorrendo lungo le pareti dell'utero e della vagina si mesce al muco e perció assume dei caratteri particolari; per la pletora si spiega pure l'aumentata secrezione delle glandule utricolari e mucipare; per l'accorciamento delle fibre muscolari nella ripiegatura del peritoneo si innalza od erige il bordo frangiato dell' imboccatura della tromba faloppiana, adattandola solto l'ovaia per impedire che l'ovicino caschi nella cavità addomiuale ed erri per cosi dire il suo cammino, il che pur troppo talrolta si verifica, tanto nella dounil come 
nelle femmine dei mammiferi, dando luogo alle cosi dette gqvidanze extra-uterine.

Nella donna, e, per quanto si assicura, anche nella femmina dei quadrumani, la caduta d' uno o più ovuli succede una volta al mese e si rende manifesta colla menstruazione. Negli altri mammiferi invece si verifica in diversi periodi, cioc̀ 0 più volte all' anno in determinate stagioni, oppure una sol volta all' anno, ed in taluni animali soltanto ad ogni due o tre anni. Pero la perdita di sangue per la vulva nelle femmine dei bruti non c̀ mai d' ordinario cosi copiosa come nella donna (1) c si limita spesso ad una semplice tumefazione ed arrossimento del vestibolo genitale con flusso di mucosità. La femmina dei bruti non permette al maschio l'accoppiamento che in questa epoca, la quale dicesi epoca o slagione degli amori. Fuori di questo tempo le femmine non sono fecondabili.

Questo fatto sembrerebbe a primo aspetto in contraddi= zione con quanto succede nella donna, la quale d'ordinario è rispettata dall'uomo durante la menstruazione e, secondo alcuni usi o riti religiosi, non può essere toccata dall' uomo se non sono scorsi cinque o sci giorni dopo che è cessata. Ma quest'apparente paradosso svanisce allorchè si considera che l'ovulo, o cellula fecondabile, ed i corpuscoli spermatici, od elcmenti fecondatori, hanno la facoltà di conservarsi per un certo tempo anche dopo che sono usciti dalle glandule in cui si formarono; i corpuscoli spermatici poi trovano uei genitali muliebri delle condizioni favorevoli a mantenersi mobili c dotati d' attività fecondatricc per molti

(1) Alcuni medici dell'antichitd credevano che il flusso menstruo avesse lo scopo di preservare la donna dagli ardori proprii alle femmine dei bruti nella stagione degli amori. Questo flusso di materia peccans ricevelte per tanto anche il nome di purga. La menstruazione dura nella donna alcuni giorni, la durata del flusso c la quartiti di sangue che esce ad ognl menstruazione varia assai sccondo il clima, la razal, l' età, il temperamento, il genere di vita. i costumi ece. 
giorni, di modo che sebbene l'accoppiamento dell'tomo colla donna si verifichi d'ordinario negli intervalli fra una menstruazione e l'altra, pure i coiti riescono fecondi dappoichè o lo sperma appena eiaculato s' incostra con un ovulo caluto riell' utero gia da qualche giorno, ciò che sembra il caso più co'mune, oppure i filamenti spermatici si trattengono nell'utero finchè vi arriva l' ovicino e lo fecondano. Comunemente si dice che la donna è sempre fecondabile, e ciò fino af un cerlo punto è vero, in quanto che il periodo di tempo clie divide la caduta d'un ovicino dalla seguente (una menstruazione dall' altra) è cosi breve che non basta a far perdere allo sperma od agli ovuli le loro rispettive attiviti; mentre che nei bruti, massime nei più voluminosi, il periudo di tempo che corre fra una stagione degli amori e la seguente è si lunga che i coili non possono essere fecondi se non in quest'epoca che ha la durata di alcuni giorni.

Vuotatosi il follicolo del suo contenuto (ovulo col cumulo ooforo e liquido albuminoso) si presenta in suo luogo alla superficie dell'ovaia una specie di ulcera caliciforme a bordi irregolari.Ben presto la cavità di quest'ulcera si riempie totalmente pel corrugarsi della membrana vascolare e per proliferazione di cellule sotto la membrana granulosa od epitelio interno del follicolo. Approssimandosi i bordi e saldandosi fra loro si cliiude l'ulcera, e siccome durante questo processo si sono aumentate le pieglıe'della membrana vascolare e le cellule di nuova formazione sotto la membrana granulosa hanno subita una metamorfosi adiposa e si sono colorate in giallo, in luogo del follicolo si osserva un tumoretto giallo, detto corpo luteo, che nello spaccato si presenta come una cisti a pareti assai grosse (sostanza corticale del corpo luteo, composta di cellule della membrana granulosa e di cellule di nuove formazione che hanno subita la metamorfosi adiposa) limitanti una piccolissima cavità nella quale si trova un liquido albuminoso, giallognolo, piuttosto torbido, poiche tiene in sospensione molte cellule identiclie a quelle della membrana gramulosa c 
falvolta perfino dei globuli rossi di sangue con coaguli fibrinosi.

In prosieguo le cellule che costituiscono la sostanza corticale si fauno più colorate per aumento del pigmenlo interno, di modo che i corpi lutei cangiano col tempo di colore, e da gialletti cle erano in principio si fanno di colore giallo aranciato, indi di color rosso viro o cinabro.

Il corpo luteo ha un periodo di evoluzione ed uno d'involuzione. Il primo incomincia subito dopo lo scoppio del follicolo ed ha una diversa durata, e quindi è diverso anche il suo sviluppo, secondo la sorte toccata all'ovulo che ue è uscito. Se questo non velue fecondato, il corpo luteo dopo d'avere raggiuuto un certo volume si arresta ed incominciano in esso lo metamorfosi regressive che finiscono colla sua totale scomparsa. Nella douna vergine pare che l'esistenza transitoria del 'corpo luteo non oltrepassi il mese, di modo che all'epoca della meustruazione non c̀ più riconoscibile il corpo luteo della menstruazione aptecedente. Ben altrimenti succede állorquando l'ovicino incontrandosi collo sperma viene fecondato e si sviluppa nell' utero;allora l'ovaia partecipa dello stato congestivo gencrale dellapparecchio genitale, nel follicolo aperto si verifica una specic di stato infiammatorio seguito da copiosa produzione di cellule solto lo strato granuloso, il corpo luteo acquista grandi dimensioni (per esempio nella giovenca arriva talvolta ad occupare quasi tutta l'ovaia) ed allora la sua involuzione non giunge mai alla totale scomparsa. E perciò le ovaic delle femmine che hanno partorito più volte presentano una superficic irregolare e contengono diversi corpi lutei ridotti alle minime proporzioni, ma pur sempre inteusamente colorati in rosso bruno.

Ciò che ì singolare si è clic scompare o viene assorbita la parte più superficiale del corpo luteo e rimane la più profonda, it clie ha fatto credere ad un'internarsi del corpo luteo ilello stroma dell'ovaia.

Nell'interno de'corpi lutei del periodo d'inyoluzione si tro- 
vano delle cellule contenenti dei granuli intensumente colorati ed una sostanza cristallina non ben determinata, la quale fino a poco tempo fa si riteneva rappresentata dall'ematoidina, mentre per le recenti ricerche dei professori Piccolo e Licben di Palermo, non clse per quelle quasi contemporanee di flolm ne viene negata l'identità.

Scbbene il clima abbia forse la maggiore influenza sulla più o meno precoce pubertà, pure non sempre la donna di diversa stirpe o razza si acclimatizza per riguardo alla menstruazione. Durante la mia dimora in Polonia ho potuto più volte constatare il fatto che le ragazze ebree della Polonia e della Russia, scbbene appartenenti a famiglic stabilite da secoli in quelle contrade settentrionali, continuano ad essere menstruate in età assai più giovane delle cattoliche: Ciò dipende probabilmente dalla tenacità degli Isracliti polacchi nell'osservanza del loro rito, specialmente per ciò che riguarda l'alimentazione ed il vivere famigliare.

La seguente tabella di Raciborski ei dà un'idea dell'influenza che esercita il clima sulla prima comparsa dei menstrui.

\begin{tabular}{|c|c|c|c|}
\hline LUOGO & $\begin{array}{l}\text { LATITUDINE } \\
\text { GEOGRAF. }\end{array}$ & $\mid \begin{array}{c}\text { TEMPERATURA } \\
\text { MEDIA }\end{array}$ & $\begin{array}{l}\text { ETÀ MEDIA ALL'EPO- } \\
\text { CA DELLA PRIMA } \\
\text { MENSTRUAZIONE }\end{array}$ \\
\hline $\begin{array}{l}\text { Marsiglia } \\
\text { Lione } \\
\text { Varsavia } \\
\text { Mancliester } \\
\text { Stoccolma } \\
\text { Lapponia }\end{array}$ & $\begin{array}{l}43^{\circ} 18^{\prime} \\
46^{0} \\
52^{0} 13^{\prime} \\
53^{0}-29^{\prime} \\
59^{\circ} \\
65^{0}\end{array}$ & $\begin{array}{l}+14,1^{\circ} \mathrm{c} \\
+11,6^{\circ} \mathrm{\nu} \\
+\quad 7,5^{\circ} \mathrm{\nu} \\
+\quad 8,7^{\circ} \mathrm{\nu} \\
+\quad 5,7^{\circ} \mathrm{\nu} \\
+\quad 4,0^{\circ} \mathrm{m}\end{array}$ & $\begin{array}{l}13,011 \\
14,492 \\
15,083 \\
15,191 \\
15,590 \\
18,\end{array}$ \\
\hline
\end{tabular}


Si conosce per gli esperimenti istituiti e ripetuti da diversi autori che negli animali mammiferi uon è assolutamente uecessario per la fecondazione delle uova l'accoppiamento materiale del maschio colla femmina, bastando che lo sperma venga portato allo stato fresco nei genitali femmiuili; ciò che si oltenne appunto injettando per mezzo di una siringa lo sperma di un cane nella vagiua di una cagna durante gli ardori, la quale diventò gravida e partori dei cagnolini perfettameute sviluppati. Questi esperimenti, quelli di piscicoltura e di fecondazione delle uova di rana, come pure alcuni fatti ben coustatati di gravidanze uelle donne per sperına umano injettato artificialmente o durante coiti in istato di. deliquio provano pure come nou sia necessario per la fecondazione quello stato di voluttuoso eccilamento che si verifica durante la copula.

In condizioni normali la luogo la copula, il cui scopo fiuale c̀ l'immissione della sperma nei genitali muliebri; il cle $10 n$ può verificarsi senza l'erezione. Eckhard ed altri considerano l'erezione come l'effetlo di un' attività nervosa centrifuga (eccilamento) dei così detli nervi erigenti, che direltamente o per mezzo di gangli produce uua paralisi de'nervi vasomotori delle arteric del pene, per cui queste si dilaterebbero per la perdila di tono delle loro pareti e darebbero accesso a nagggior quantila di sangue che va a ricmpire e disteudere i corpi cavernosi. Questi nervi erigenti potrebbero essere eccilati direltamente dai centri nervosi (cervello, midollo spinale) oppure per riflesso di eccitamenti periferici clıe movercbbero al midolio spinale per la via del nervo pudendo.

In maucanza di altra teoria accettiamo per ora questa, la quale potrà essere confermata sol quando si arriverà a togliere per sempre Ia possibilitá dell'erezione del penc in un animale col laglio dei nervi erigenti; seblene una tale teorica non cluin- 
da il varco ad alcuni dubbi dal momento che sappiamo come si possa produrre l'erezione del pene in circostamze c condizioni assai disparate fra loro, e specialmente per condizioni meccaniclie.

Quando le vescichette seminali si trovano distese da molto sperma ivi raccolto, una compressione un po' continuata su di esse puô essere seguita da un lento e scarso flusso spermatico per l'uretra, come succede spesse volte nelle defecazioni difficili. D'ordinario però l'uscita dello sperma dalle vescichette è sempre preceduta dall' erczione dcl pene ; per contrazione delle vescicole spermatiche e dei dotti deferenti lo sperma viene a raccogliersi nella porzione membranacea e bulbosa dell'uretra e finalmente viene spinto (ejaculato) per le contrazioni ritmiche convulsive del muscolo bulbo-cavernóso associate a quelle dei muscoli ischio-cavcruosi, della prostata ed altri.

LUOGO DOVE I FILAMENTI SPERMATICI INCONTRAF̃O GLI OVULI

Siccome nelle femmine dei mammiferi uccise poco dopo che vennero coperte dal maschio si trovarono i filamenti spermatici non solo nell' utero e nelle trombe ma anche sulle ovaie, si ritenne che i filamenti spermatici penetrassero nei follicoli per fecondarvi gli ovuli contenuti, e che questa fosse la causa dello scoppio del follicolo e della conseguente uscita dell' ovulo. Noi peró sappiamo, per quanto si disse antccedentemente, clie nella donna come negli altri animali la caduta degli ovicini è spontanea e periodica e perciò ora traltasi unicamente di delerminare dove avriene l' incontro del liquido fecondatore cogli ovicini. I casi non molto rari di gravidanze interstiziali, tubariche, ovariche ed addominali ci dimostrano che il contatto fecondo dei filamenti spermatici cogli ovuli può verificarsi anche fuori dell'utero, e siccome l'avienuta fecondazione non implica l'aresto immediato dell' ovicino nel'suo movimento progressivo verso 
l' utero, così è nolto probabile clie iu condizioni fisiologiche l' incontro dei filamenti spermatici cogli ovicini abbia luogo nella tromba faloppiana o sull' ovaia, ma che l'ovicino dopo l'avrenuta fecondazione, invece di fissarsi alla mucosa della luba nel punto dore venne fecondato, continui il suo cammino discendendo verso l' utero, che è il suo ricettacolo fisiologico. Ivi trova le condizioni più favorevoli al suo ingrandimento, non clie la possibilita di uscire quando ha raggiunto lo sviluppo necessario, dappoichè durante la gestazione le pareti dell' utero si fanno assai robuste, per sviluppo del tessuto muscolare, in proporzione dell' aumento in volume e capacità. E cosi allorquando il felo è giunto a maturanza le pareti muscolari di questo ricettacolo, mediante forti.e continuate contrazioni lo espellono vincendo le resistenze e gli ostacoli rappresentati dalla strettezza dei canali e delle àperture per le quali deve uscire.

Qualche rara volta l'ovicino si arresta (prima o dopo avveuuta la fecondazione) e si sviluppa fuori della cavità uterina ed allora si hanno le cosi dette gravidanze extra-uterine, le quali mettono quasi sempre in pericolo la vita della madre e del frutto del concepimento. Secondo la località ove l'ovicino si arresta e si sviluppa distinguonsi le gravidanze extrauterine in interstiziali (1), tubariche ed ovariche. Quando poi l'ovicino non viene raccolto dalla tromba faloppiana e cade, dopo essere stato fecondato, nella cavità addominale ove si sviluppa, allora si ha la così detta gravidanza extrauterina addominale.

Non volendo nè potendo noi negare i fatti, ammettiamo la possibiliti della gravidanza ovarica, ma l' interpreliamo in IIII modo diverso da quello che si credeva una volta dagli Embriologi, sccondo i quali i Gilamenti spermatici dovevano

(1) Sono assai rari i casi ben constalali di gravidanza inlerstiziale prodotla dall' arresto e sviluppo dell' novo nel tratlo inferiore della tuba compreso nello spessore delle pareti dell' utero. 
sempre penetrare nell' ovaia per fecondarvi gli oricini contenuti nei follicoli, ciò che sembra inutile se uon impossibile, massime nei mammiferi, stante lo spessore delle pareti dei follicoli. Troviamo per altro possibile clie i filamenti spermatici semoventi sulla superficie dell' ovaia penetrino ill un follicolo nel momento che questo si apre, per cui l'ovicino viene ad essere fecondato ancora nella cavità del suo follicolo od appena che ne è uscito e trovasi arrestato sull'ovaia da in impedimento qualunque; ivi poi circondandosi d' un cercine o rialzo di connettivo acquista tali aderenze e rapporti da svilupparsi come se fosse nell' utero. śiccome il cercine di connettivo a poco a poco s' innalza e si chiude sopra dell' uovo e questo sacco di nuova formazione si continua alla base collo stroma dell' ovaia, cosi ne venne che per molto tempo dopo la scoperta dei follicoli del Graaf le gravidanze ovariche erano citate come prove che la fecondazione succedeva nel follicolo e non già fuori dello stesso.

Prima di abbandonare l' argomento delle gravidanze extrauterine mi piace di ricordare due fatti e sono: $1^{\circ}$ che l'oviciıo fecondato e che si sviluppa, sia nella cavità addominale come sull'ovaia o nella tuba, agisce sulla superficie che tocca come un corpo straniero provocando una specie di stato illfiammatorio ; i tessuti circostanti si gonfiano e formano un cercine che sempre più s'innalza e finalmente si chiude formando un sacco come ho già descritto parlando della gravidanza ovarica ; $2^{\circ}$ che nelle gravidanze extrauterine l' utero partecipa più o meno allo stato congestivo; e per ciò s'ingrossa per aumento di spessore delle sue pareti, la sua mucosa diventa tumida ed i vasi sanguigni della stessa si sviluppano e si dispongono ad anse le quali dovrebbero intrecciarsi colle anse del chorion vascolare di cui si circonda l'uoro in via di sviluppo.

Comunemente nella donna si apre un solo follicolo ad ogni menstruazione e perció la donna d'ordinario netle alla luce 
un figlio alla volta. Nou essendo per altro assai rari i casi di parti gémini e verificandosi pure, sebbene assai più di rado, dei parti trigemini e quadrigemini (1) fa d' uopo ammettere che alcuni follicoli contengano due o più ovuli oppure, ciò che sembra più probabile, che talvolta si aprano due o più follicoli contemporaneamente. Avvi per altro anche un' altra possibilità ed è che lo sperma fecondi un' ovulo già caduto nell' utero e si conservi attivo per fecondare un ovulo clie esce dal follicolo alla menstruazione susseguente. In tal caso si ha la superfecondazione.

Nelle gravidanze cosi dette composte gli ovuli possono svilupparsi nell'utero ad una certa distanza l'uno dall' altro chiusi in sacchi separati e mantenendosi sempre indipendenti, oppure gli ovicini sebbene divisi vengono involti da un sacco comune. In questo caso è molto facile che l' uno includa una parte dell' al tro dando origine a delle mostruosità.

Per superfetazione s' intende la fecondazione di un uovo due, tre, o più mesi dopo che è incominciata la gravidanza. In questi casi o i feti nascono contemporaneamente con manifesta differenza di sviluppo, o Ia nascita di essi succede alla medesima distanza di tempo che s'interpose fra l' una e l' altra fecondazione. Simili casi sembrano verificarsi d' ordinario in donne con utero diviso in due distinte cavità e che perciô dicesi utero bicorne.

CAMBLAMNTI DELL' Jovo Dupo L' USCLTA DAL FOLLICOLO

L' ovulo umano appena uscito dal follicolo è visibile ad occhio nudo sotto la forma d'un piccolissimo corpuscolo sfer ico, a contorni non sempre regolari e di color bianco grigio (2). Veduto al microscopio vi si distinguono la membrana vitelli-

(1) Dalle statisticlie si rileva che la frequenza dei parti gemelli sta a qnella dei parti semplici come 1: 70-\$0, i trigemini iuvece sono rarissimi vioc neila proportione di 1:7000-8000.

(2) Il diametro dell' ovulo umano maturo varia lra $0,08-0,1^{\prime \prime \prime}$; fer 
na a doppio contorno, il vitello granuloso interno e la vescicola germinativa contenente la macula germinativa. 'Tutto all' intorno ì circondato dal disco ooforo a bordi irregolari formato dalle cellule della meinbrana granulosa; le cellule che toccano direttamente la membrana vitellina sono oblunghe e disposte normalmente alle tangenti dei punti della superficie dell'uovo, di modo che ne risulta la forma d' una rosetta come si vede nella figura dell'atlante.

Non si conosce alcuu che intorno ai cambiamenti che subisce l'ovulo umano appena uscito dal follicolo e nei primi momenti che seguono l'avvenuta fecondazione, stante le difficoltà o piuttosto l'impossibilità di avere il necessario materiale fresco di osservazione e studio.

Perciò noll essendo state ancora osservate le prime fasi di sviluppo dell'uovo umano (1) noi dovremo studiarle nelle uova degli animali. E siccome i mutamenti evolutorii delle uova sono differenti nei diversi animali noi studieremo questi processi secondo le osservazioni fatte sulle uova di animali vertebrati mammiferi, e ricorreremo ai fatti osservati nelle uova di altri animali vertebrati od invertebrati ogui volta che si crederà conveniente ed utile per chiarire i dubbi e per generalizzare alcune leggi che seguono i movimenti evolutorii in tutte le uova.

L'ovulo impiega un certo tempo (alcuni giorni) a percorrere la tuba per portarsi nell' utero. Pare che proceda innanzi con un movimento rotatorio, il quale non è dovuto nè alla gravità nè alle contrazioni peristaltiche della tuba, ma piuttosto alle vibrazioni delle ciglia dell’ epitelio che tapezza la mucosa di questi canali ed anclıe alle rotazioni proprie del vitello.

la diffieoltà di isolare la vescichetta germinativa dal tuorlo non si è potuto aneora determinarne le dimensioni preeise.

Per avere un'illea della grandezza dell'ovulo umano figuriamoei un punto segnato sulla earta colla punta funissima di una matita.

(1) Le uova umane osservate finora in via di sviluppo presintarauo già le forme embrionali: 
Cone l'uovo di gallina e degli altri ovipari anche l'uovo dei nummiferi nel suo decorso attraverso alle tube si circonila d' uno strato di sostanza albuminosa o inucosa, mentre il disco ooforo scompare a poco a poco per sfaldamento delle cellule clic lo compongono. Oltre a ciò l'ovulo s' ingrossa per unore clie assorbe dalla mucosa tubarica; tutto ciò avvienc indipendentemente dalla fecondazione.

E cosi pure ancor prima della fecondazione nell'uovo maturo appena uscito dal follicolo si osservano de' fenomeni singolari di movimenti nel vitello, il quale eseguisce delle rotazioni periodiche intorno alla vescicola germinativa e di tanto in tanto si contrac in un senso o nell'altro in modo che assume diverse forme (ovali, piriformi, irregolari con processi) per riprender poco dopo la forma primitiva od acquistarne un'altra. Questi movimenti autonomi che hanno per risultato de' cambiamenti di forma si considerano da taluni come analoghi assai ai movimenti amibiformi proprii del protoplasma di altre cellule animali. Dalle osservazioni che ebbi occasione di fare sulle uova di gallina appena tolte dall'ovidutto mi consta che il vitello o cicatricola eseguisce delfe contrazioni ritmiche analoghe a quelle dei punti pulsanti dei parameci ed altri infusorj. Le contrazioni del vitello dell' novo terminano colla separazione di alcune porzioni dal rimanente, e siccome questo processo verificasi d'ordinario prima ad un polo ed in seguito al polo opposto, le masse che si staccano dal corpo del vitello appellansi masse o globuli polari. Queste lianno un aspetto particolare diverso dal vitello da cui si sono staccate, cioè sono trasparenti, jaline e sembrano formate come da una sostanza mucosa che siasi separata dal vitello, il quale invece si è fatto più opaco e denso. Si direbbe quasi che il vitello stringendosi in se stesso abbia. prodotto una separazione dei granuli dal liquido o sostanza jalina intergranulare, la quale scparandosi dai primi diede origine ai globuli polari. 
Durante i processi or ora descrilti scompare la vescicola germinativa col suo nucleo; non si sa in qual modo e che ne avvenga (1). La comparsa di un nucleo ^el centro del vitello ì la scena finale di tutta l'azione propria dell'ovicino quando venne fecondato; in caso contrario rallentandosi i movimenti di rotazione ed amibiformi del vitello l'uovo si spappola e scompare. Alcuni autori sostenguno per altro che non in tulli gli ovuli dei diversi animali la comparsa del nucleo segni l'avvenuta fecondazione cioè l' iıdividualizzazione della cellula. Tutti però ammettono che una volta avvenuta la fecondazione il protoplasma subisce un cambiamento che può essere paragonato ad una coagulazione, poichè si divide in una massa centrale densa ed opaca, ed in un liquido limpido che s' interpone tra il coagulo centrale granuloso e la membrana vitellina. Trovandosi l' ovulo in contatto colla mucosa della tromba 0 dell' utero assorbe nuovo liquido che si raccoglie sempre più intorno alla massa centrale vitellina, la quale subisce il cosi detto processo di segmentazione totale o parziale. Questo si verifica nel modo seguente: la massa vitellina presenta da principio un solco equatoriale, il quale si fa sempre più profondo finchè giunge a dividerla iı due segmenti emisferici che si staccano subito l'uno dall'altro ed assumouo una forma più o meno sferica; (2) poco dopo si presenta in ognuna delle due

(1) Sono divise lc opinioni degli autori intorno alla scomparsa della vescichetta germinativa ed alla divisione del vitello o protoplasma in un coagulo eentrale ed in un liquido sieroso periferico, dappoichẻ aleuni rltengono ehe ciò avvenga soltanto nelle uova fecondate ed altri eredono che sia un fenomeno indipendente dalla feeoudazione.

(2) Negli ovuli in eui è visibile il nueleo eentrale alla massa vitcllina si osserva che il proeesso di segmentazione ineomincia a manifestarsi nel meleo, il quale trovasi gia diviso mentre il soleo equatoriale della massa vitellina non è ancora giunto a dividerla in due.

Negli ovuli di alcuni animali il processo di segmentazione assomirlia 
masse un solco equatoriale che le divide iu due e cosil la masSa vitellina viene ora ad essere rappresentata da quallro masse sfericle centrali circondate dal liquido trasparente. Questo processo di segmentazione si conlinua in seguito sempre coll' esponeute due, finchè tutta la massa vitellina trovasi ridotta in tanti piccoli globuli sferoidali del diametro di circa $1 / 100$ di linea, in cui si distinguono una membranella involgente, un contenuto fino granuloso assai denso, nel centro del quale si osserva un nucleo trasparente. E con ciò il vitello viene ridotto ad un ammasso di vere cellule.

Intanto il liquido trasparente che circondava le masse di segmentazione, e che durante il processo si è aumentato per endosmosi, interponendosi tra una massa e l'altra, si raccoglie a poco a poco nel centro e quando le masse risultanti dal processo di segmentazione hanno acquistato il carattere di cellule, vengono spinte dal liquido verso la periferia e contro la membrana vitellina in modo da formare una specie di strato epiteliale che la tapezza internamente. Questo strato di cellule nucleate granulari dicesi blastoderma o membrana germinaliva.

PRIMO CHORION VILLOSO

Negli animali mammiferi, e probabilmente anche nell' uomo, durante questi cambiamenti si verifica un aumento in volume per continuo assorbimento di liquido e la zona pellucida si assoltiglia e si copre di tanti processi filiformi o villi mediante i quali si lissa alla mucosa dell' utero o s'inocula, come vorrebbe Pllüger, nelle ferite risultanti dalle lacerazioni per le quali è uscito il sangue menstruo.

piuttosto ad una gemmazione, perehè alla superficic della massa vitellina sferica si presenta una gemma, la quale a poco a poco $s^{2}$ ingrossa c finalmente si staeca quando ha riggriunte le dimensioni della massa generatriec; e questo é un escmpio di soslituzione del processo di gemmazione a quello di seissione che abbiamo veduto verificarsi anele nellat generazione agamn e che verlrenon epesso ripetersi nella genesi dei uessuti. 
Uila volta che la membrana vitellina si è coperta di villosità prende il nome di chorion villoso, il quale, come abbiamo già veduto, ¿̀ tapezzato interamente dalle cellule blastodermiche, risultanti dal processo di segmentazione del vitello, disposte l'una presso l' altra in modo da formare un sacco concentrico all' interno del chorion; la cavitù dell' uovo è ripiena di un liquido che va sempre più aumentando per assorbimento. Questo liquido ha l' aspetto d' uma fina emulsione poichè tiene in sospensione molti granelli, che alcuni considerano come il detrito di masse di segmentazione. Tulte le cellule del blastoderma dapprima sono sferiche, in seguito si fanno piatte dal lato che tocca il chorion e sono sporgenti dal lato opposto cioc̀ verso il liquido, il quale ora si puó chiamare vilello embrionale o della futura vescicola ombelicale.

In un punto del blastoderma osservasi la cosi detta area germinaliva, cicè un cumulo a contorno circolare di cellule identiche a quelle del blastoderma. Questo cumulo sporge verso l'interno dell'uovo ed al pari del blastoderma è coperto diretlamente dal clorion. Dicesi area germinativa perchè appunto in essa o per meglio dire da essa si forma il corpo dell'embrione.

Beı presto le cellule del blastoderma e le superficiali dell' area germinativa si fanno piatte anche dal lato interno e per compressione laterale reciproca acquistano forme poligonali (d'ordinario pentagone od esagone); indi si saldano tra loro in modo da costituire una vera membrana continua, formata d' un semplice strato di cellule eccetto nel punto occupato dall' area. Intanto l' uovo s'ingrossa e le cellule del blastoderma si aumentano disponendosi in due strati.

In prosieguo si differenziano le cellule della vescicola blastodermica dello strato esterno da quelle dello strato interno; lo strato interno inferiore consta di cellule piccole, pallide e granulose con un nucleo vescicolare distinte, conlenente uno o due nucleoli, mentre lo stralo superiore consta di clementi cellulari più oscuri e ripieni di goccioline di grasso, 
nei quali diflicilmente si distingue una membrana involgente ed un nucleo.

STRATIFICAZLONE DEL BLASTODERMA; AREA OPACA E TRASPÁRENTE

Tanto il processo pel quale le cellule del blastoderma si saldano tra di loro in modo da costituire una vera membrana, quanto la divisione in istrati distinti incominciano all'area germinativa come punto centrale e procedono verso la periferia estendendosi a tutto il blastoderma; più tardi poi fra i duc strati dell' area germinativa si vede formarsene un terzo per produzione di nuove cellule da quelle degli strati esistenti, e probabilmente piuttosto dal superiore. La divisione in tre strati, clıe d'ora innanzi noi vorremo chiamare foglietti, non oltrepassa i limiti dell' area germinativa rimanendo il blastoderma costituito da due soli foglietti, uno esterno l'altro interno.

Il foglietto mediano dell' area germinativa sembra costituito da cellule dotate d'una grande attività prolifera, sicchè in poco tempo acquista un grande spessore in confronto degli altri due e specialmente dell' inferiore od interno, il quale si conserva allo stato primordiale cioè formato di uno strato di cellule a modo d' un epitelio pavimentoso semplice.

L' area germinativa si estende mano mano che l'ovulo s'ingrossa; l' ingrandimento dell' area germinativa succede principalmente da parte del foglietto mediano, il quale sviluppandosi più in un senso che nell' altro muta la figura dell' area germinativa, che dapprima era circolare ed in seguito diventa piuttosto elittica od ovoidale.

Questa dilatazione dell'area è accompagnata da un'apparente rarefazione centrale, per cui nella direzione del suo asse maggiore si vede formarsi nel centro una figura oblunga più trasparente, che venne percio chiamata area trasparenle, mentre la porzione periferica dicesi area opaca perchè conserva la sua opaciti. Ecco quindi l' area germinativa distinta 
ill una porzione centrale trasparente, il di cui foglietto interno consta di cellule molto cliare, ed in una porzione periferica opaca. Per altro non vorrei si credesse veramente che la parte trasparente fosse piis sottile dell' altra; anzi l'osservazione microscopica di sezioni normali dell' area germinativa prora piuttosto il contrario, e quindi l' opacità e la trasparenza dipendono dalla natura diversa delle cellule che costituiscono le due aree.

Mentre l'area opaca distendendosi acquista di nuovo la figura circolare, l'area trasparente si stringe nel mezzo e prende la forma d'una suola di scarpa; e noi vedremo come in seguito piegandosi i suoi bordi in basso, cioè verso il centro dell'uovo, ed avvicinaudosi tra loro nella linea mediana ma soltanto alle due estremità (più all'anteriore che alla posteriore) assumerà la forma d'una vera pianella.

\section{NOTA PRIMITIVA}

Appena che l'area germinativa si è differenziala in una parte centrale (area trasparente) ed in una parte periferica (area opaca) si osserva nel centro della prima una linea opaca, saliente e longitudinale, prodolta da un raggruppamento di cellule blastodermiche del foglietto mediano, che solleva il foglielto superiore formando come una piega o leggiera cresta saliente alla superficie del blastoderma, la quale venne chiamata dal Baer nola primitiva perchè è la primissima traccia del corpo dell' embrione. Questa linea si allunga e si fa piì larga e piatta; in seguito presenta un solco mediano poco profoudo e longitudinale, doccia primiliva, i di cui bordi esterni s'innalzano ed all'estremità anteriore o cefalica si uniscono ad arco, mentre all'estremità posteriore o caudale si fondono tra di loro e terminano in una punta più meno acuminata a modo d' una lancetta. Il tralto intermedio della doccia primitiva presenta i bordi equidistanti fra loro. 
Per comprendere più facilmente come da un' area polare o disco convesso d'una vescicola sferica (qual' è in fatli l' area germinativa rispetto al blastoderma) si possa formarc il corpo d'un animale vertebrato, consideriamo per un momento la sezione schematica del corpo dell' nomo adulto (1). Noi vediamo duc sezioni di tubi posti l'uno sopra dell'altro, le di cui pareli constano di più strati concentrici. Questi due sistemi tubulari sono involti da un grande tubo comunc, Ic di cui pareli constano parimenti di più strati, quali sollo : i tegumenti comuni, i muscoli e lc ossa delle pareti toraciche ed addominali, il foglio parietale del peritoneo; i due tubi sovrapposti l'uno all' altro trovansi nella linea mediana e sono rappresentati: il supcriorc dal canale della midolla spinale e l' inferiore dal tubo digerente. Le pareti del canale-supcriore (canale del midollo spinale) sono formate. $1^{0}$ dall'epitelio interno, $2^{0}$ dalla sostanza grigia e bianca che coitituisce l'asse nervoso cerebro-spinale, $3^{\circ}$ dalle meningi c $4^{0}$ dalle vertcbre, che colle loro nevro-apofisi (archi vertebrali) formano degli anclli, cioc̀ le sezioni dello speco vertebrale che protegge il midollo, spinale. Il tubo inferiore, diviso ed unito contemporaneamente al superiore per i corpi delle vertebre, è formato: $1^{\circ}$ dallo strato epitclialc della muco$\mathrm{sa}, 2^{0}$ dalla mucosa stessa c sue glandule, $3^{0}$ dal tessuto fibroso muscolare c finalmente dal peritoneo; questo lubo è libero in una cavi tà detta plcuro-peritoneale, e trovasi tenuto fisso unicamente nella linea mediana dorsale dal peritonco, cle in questo punto da viscerale diventa pariclale, cioè dal rivestirc e comprendere i visceri passa a tapezzare la faccia interna delle pareti jel tronco.

In seguito a questa considerazionc schematica del corpo

(1) In questa considerazione si fa astrazione dalle estremila, quali appendici accessorie, e si pensa il tubo gastro-cutcrico scmplice e rettilineo. 
dell' uomo adulto noi comprenderemo ora facilinente fa formazione dei due tubi sovrapposti (animale e viseerale), quella degli strati concentrici che li costituiscono, ed infine la formazione del tubo comune esterno.

La piastrina cmbrionale e la doccia primitiva si allungano e s'incurvano; i margini esterni della piastrina si picgano in basso ed avvicinandosi nella linea mediana si suldano formando il tubo inferiore interno.

Lo stesso avviene per i bordi che limitano la doccia primitiva superiore, i quali s'innalzano e si saldano nella linea mediana dorsale. Il foglietto superiore dell' area germinativa viene cosi ad involgere contemporaneamente i due tubi rappresentando l'epidermide, mentre il terzo foglietto od inferiore viene chiuso nel lume del tubo inferiore in modo che rappresenta l'epitelio intestinale; e perciò queste due foglietti si chiamano, fino dal loro apparire, il primo epidermoidale animale, ed il secondo epiteliale organieo. II foglielto. mediano prende la parte principale nella costituzione delle pareti dei due tubi non che del tulo comune involgente (pareti del corpo); e per questo il foglietto mediáno dicesi motoriogerminativo, appunto perchè dallo stesso hanno origine il tessuto connetlivo nelle sue varie forme (ossea, cartilaginea, membranacea), il tessuto muscolare in generale, il vascolare ed îl tessuto nervoso ; è da osservare peró che questo foglietto mediano ben presto si divide in due strati distinti, i quali restano saldati soltanto nella linea mediana dorsale. La pagina esterna del foglietto mediano origina le pareti del tronco e del tubo superiore, per cui si potrebbe dire per eccellenza la vera pagina animale, mentre la pagina inferiore forma le pareti dell' intestino cogli annessi organi glandulari e perciò potrebbe chiamarsi pagina vegelativa od organica.

Siccome il saldamento dei bordi del tubo inferiore arviene a poco a poco, cosi il lume dell' intestino rimane in comunicazione colla cavità del blastoderma contenente il vitello embrionale. 
C.ORd DORSALE E VERTERRE PRHMTIVE

Come abbiamo veduto l'area germinativa per neo-formazione di cellule si divide in tre foglietli, dei quali il superiore od esterno e l' inferiore od interno si continuano oltre il limite dell'area germinaliva nei due foglietti del blastoderma, mentre il foglietlo mediano assai grosso nou varca i limiti dell'area stessa, e presenta apricna formato uno spessore massimo nel centro, dal quale punto va mano mano assottigliandosi verso la periferia.

Sappiamo pure come per differenziamento delle cellule cmbrio-plasticle disposte nella parte centrale dell' area opaca si formi l'area trasparente, c per rarefazione lineare mediana di quest' ultima si sviluppi la doccia primitiva, i di cui margini sporgenti innalzandosi e piegandosi verso la linea mediana dorsale si dispongono a trasformarla in un canale chiuso. Appena che si è formata la doccia primitiva le cellule dello strato mediano che trovansi immediatamente sottoposte, si dispongono in serie formando un cordone o stiletto cilindrico che termina assottigliato alle due estremità. Formatosi per raggruppamento di cellule embrio-plastiche strettamente unite fra loro, questo stiletto, che dicesi corda dorsale, si distingue facilmente per la sua opacità e resistenza anche attraverso agli altri organi e tessuti embrionali sovrapposti. La corda dorsale è il primo rudimento della colonna vertebrale, mentre la doccia primitiva rappresenta l'inizio dell'asse cerebro-spinale. Come moltecellule del foglietto mediano hanno costituita la corda dorsale al di sotto della doccia primitiva, cosi le cellule embrio-plastiche del foglietto mediano laterali alla corda si raggruppano e formano dapprima due lamine rettangolari oblunghe, una a destra l'altra a sinistra, le quali si scindono subito dopo trasversalmente, costituendo tante masse subprismatiche ai lati della corda. Come ognumo di léggieri comprende queste masse rapprescutano le tracce delle meta lateralidelle vertebre 
primitive, delle quali se ne formano in principio cinque o sei per ciascun lato, e precisamente nel mezzo tra l'estremità cefalica e la caudale dell'embrione. Pare che le prime vertebre a comparire sieno le cervicali inferiori e le prime toracicle. Kölliker e Remak sarebbero invece d'avviso clie le prime vertebre a formarsi sieno le cervicali superiori, c che tutte le altre che si formano in seguito, si sviluppino dictro delle prime, mentre Buer sosticne che la nuova formazione di altre vertebre succede tanto verso la parte cefalica come verso la parte caudale.

Queste masse subprismatiche ingrossandosi vengono a poco a poco ad abbracciare superiormente la doccia primitiva ed in basso la corda dorsale, dappoicliè questa che si è formata per la prima si sviluppa più lentamente. Si comprende facilmente come le vertebre primitive sollevandosi per circondare la doccia primiliva debbano imalzare il foglielto corneo" epidermoidale, ed adattandosi colla loro faccia interna alla doccia primitiva, la quale intanto ha presa la forma di un canale quasi cilindrico, debbano farsi concave al lato interno.

Durante questi processi di formazione delle vertebre, le cellule embrio-plasticlıe dello strato mediano laterali alle masse subprismatiche costituiscono duc lamine, lamine o piastrine lalerali, il di cui spessore va diminuendo verso la periferia. Subito dopo la loro formazione queste piastrine si dividono in due pagine, l'una superiore e l'altra inferiore, lasciando tra loro uno spazio. La divisione incomincia in vicinanza delle vertebre primitive e procede verso la periferia. Le pagine per altro rimangono saldate tra di loro tanto verso la linea mediana come alla periferia. Lo spazio che va sempre più ampliandosi per l'allontanarsi d'una pagina dall'altra costituisce la cavità pleuro-peritoneale. La pagina superiore rimane sempre aderente al foglietto corneo, I'inferiore al foglictto mucoso intestinale; alla periferia questi quattro strati rimangono fra loro saldati come in una sola membrana stratificata che si continua col blastoderma. 
Dalla pagina superiore si sviluppano i tegumenti comuni ed i muscoli della parete del tronco; dall' inferiore le pareti del tubo gastro-enterico, meno l' epitelio e le glandule che sono produzioni del terzo foglietto o mucoso-intestinale.

Negli spazii triangolari risultanti fra gli spigoli superiori esterni delle masse subprismatiche ed i bordi interni delle piastrinc Iaterali si raccolgono duc ammassi oblunghi di ccllule embrio-plastiche del foglietto mediano per costituire i reni primilivi o corpi del Wolf, mentre gli spazii triangolari inferiori tra le vertebre primitive e Ic piastrine Iaterali diventano due canali sanguigni, le aorle-primitive.

In prosieguo di sviluppo le masse subprimatiche prendono la vera forma di vertebrc. I processi superiori diconsi nevroapofisi perchè rinchiudono l' asse cercbro-spinale; le masse subprismatiche d'un Iato fondendosi con quelle del lato opposto rinchiudono la corda dorsale, la quale nei vertebrati superiori, come nell'uomo, scompare presto incominciando l'involuzione dai tratti compresi nei corpi delle vertcbre.

PRIMA FORMAZIONE DELL' INTESTINO E DELIA VESCICOLA OMBELICALE.

Come avviene alla parte dorsale che si forma dapprima una doccia limitata da due rialzi laterali, i quali innalzandosi e piegandosi l' un verso l'altro constituiscono a poco a poco un canale chiuso, cosi succede purc alla parte addominale. Le lamine parietali del secondo foglietto si piegano a poco a poco e si avvicinano coi loro margini liberi verso la linca mediana inferiore, e con ciò il foglio mucoso insicme alla pagina inferiore del foglictto medio costituisce la parete di un grande canale, che $\grave{c}$ la porzione digerente del canalc intestillale primitivo, il quale termina a fondo cieco tanto all' estremiti caudale come alla cefalica. Il processo di chiusura di questo canale avvicne prima alle estremita (cefalica e caudale) di modo che la cavitạ costituita dell' intestino rimáne nella sua porzione centrale in comunicazione dirella 
colla cavita interna del blastoderma. Cosi s'inizia la separazione dell' embrione dal resto del blastoderma, clıe a poco a poco si costituisce in una grossa vescica contenente la parte fluida residua del vitello; questa vescica è pertanto disposta contro la faccia addominale dell' embrione e comunica colla cavità dellintestino primitivo mediante un'apertura, la quale dapprima è larga ed ampia quanto è lungo il corpo dell' embrione, ma a poco a poco va stringendosi in ragione che si avvicinano i bordi delle pareti dell' intestino e quelli delle pareti del corpo; questa è la cosi detta vescicola ombelicale a vitellina, la di cui porzione stretta, che a poco a poco si allunga e forma una sperie di un collo o peduncolo, dicesi dot to omfalo-mesenterico. Negli uccelli la vescicola ombelicale comprende tutto il tuorlo giallo, per cui è assai voluminosa in confronto dell' embrione e serve non solo a fornirgli il materiale di nutrizione, ma a modo di un soffice cuscino (su cui l'embrione riposa e da cui viene in gran parte circondato) lo protegge dagli urti meccanici ed impedisce i rapidi cangiamenti di temperatura; nei mammiferi invece questa vescicola ha il significato di un serbatoio di sostanza nutritizia soltanto nei primordii della vita embrionale, ed una volta che si è sviluppato il chórion vascolare clie mette l' embrione in intimo rapporto colla madre, la vescicola ombelicule resta allo stato rudimentale mantenendosi in continuazione coll'intestino nella regione dell'ombelico primitivo mediante un canale che in seguito si oblitera e si riduce a cordone (1).

(1) Negli nccclli, comc p. e. nei polli, la vescicola ombelicalc poco prima chc il pulcino esca dall'uovo viene rinchiusa nel caro addominale, ove si trova al davantied al di sotto del pachetto intestinalc c mediante un pcduncolo pervio sta in comunicazione coll' intestino teuuc circa a mctà dclIo stesso; nei pulcini nati da due o tre settimanc si osscrva in questo punto dell' intestino una piccola appendice cieca, cilindrica, tcrminante in un bottone giallo, chc è appunto il residuo della vescicola ombelicale la quale venne assorbita.

Nci mammiferi, come ncll'uomo, all'cpoca della nascita il dotto della' vescicola ombelicalc si trova ai lati del cordone ombclicalc, e la vescicola piriforme giace sulla faccia dorsalc della placenta fetale. 
Non è peró a credere clie il materiale nutritizio della vescicoli onbelicale si versi nell' intestino per essere ivi digerito el assorbito come succede degli alimenti nell' animale formato ; dappoicliè quando incominciano a costituirsi le pareti dell'intestino e del corpo dell' embrione, si sviluppa il cuore da cui si dipartono, come vedremo più tardi, dei vasi. (arterie) i quali ramificandosi formano una rete sulla vescicola vitellina da cui lıanno origine altri vasi (vene) che tornano al cuore, e cosi per mezzo del sangue clie circola in questi si stabilisce lo scambio di materiale alimentare fra il vitello ed il corpo dell' embrione.

FORMIZIONE DEL SEGONDO E TERZO GHORION (SIEROSO E VASGOLARE) ALLANTOIDE E PLAGENTA

Sappiamo che la membrana vitellina durante il processo di segmentazione perde l' aspetto liscio e levigato per la produzione di tante appendici o villi sulla sua superficie libera, mediante i quali l'ovicino si fissa alla mucosa uterina. Una volta costituito il blastoderma in due foglietti, lo esterno di essi, detto anche sieroso, rimane aderente alla membrana vitellina, . mentre l' interno costituisce le pareti della vescicola ombelicale. Ora il foglietto esterno del blastoderma produce all'infuori dei processi, i quali penetrano in quelli della membrana vitellina, oppure rivestendosi della membrana vitellina nei punti sprovisti di villi, costituiscono il così detto chorion villoso secondo o sieroso ; questo manca pure di vasi sanguigni, ma serve a fissare sempre più l' uovo ed a metterlo ill più stretto rapporto colla mucosa dell'utero; ̀̀ anche assai probabile che per mezzo di questi villi, per semplice fenomeno di diffusione, si verifichi uno scambio di materiale tra la madre c l'uovo con prevalenza per quest'ultimo di introiti anzichè di perdite.

$\Lambda$ comprendere ora come questo chorion villoso diventi vascolare fa d'uopo premellere duc falti, l'uno fisiologico, anatomico l' altro. $\Lambda$ ppena iniziala la vita embrionale (ciò clıe succede colla comparsa delle prime parli rudimentali del cor- 
10 embrionale) devono naturalmente verificarsi dei processi d'ossidazione proprii agli organismi animali, e quindi sorge la necessità di un organo destinato ad eliminarne i prodotti. Questi organi escretori per eccellenza, detti corpi del Wolf o reni primitivi, si vedono appunto sorgere dalle cellule embrio-plastiche del foglietto mediano, che rimangono isolate per la formazione delle vertebre primitive e delle lamine parietali, come di sopra abbiamo indicato. Questi ammassi di cellule embrio-plastiche costituiscono due corpi oblunghi disposti ai lati della colonna vertebrale addominale e pelvica, posteriormente all' intestino, coperti dal foglietto esterno e quindi dai tegumenti comuni.

Da questi ammassi oblunghi di cellule si sviluppano due veri organi glandulari, costituiti da tanti tubulini trasversali che terminano da un lato con un fondo cieco rigonfiato e dall'altro sboccano in un condotto comune rettilineo, detto dollo escretore comune del corpo del Wolff. I due dotti escretori convergolio in basso e sboccano in una vescicoletta piriforme, la quale secondo alcuni sarebbe un diverticolo sporgente all' innanzi dal fondo cieco caudale dell' intestino. La parete di questa vescica, come tutto l' apparecchio uro-poetico embrionale, è riccamente fornita di vasi, di modo che si presenta alla commessura inferiore delle pareti addominali (regione pubica) sotto l'aspetto di un bottone piriforme rosso, cavo e pieno dei prodotti di ossidazione eliminati per i corpi del Wolff. Questa vescichetta a pareti vascolari, che dicesi allantoide, si sviluppa assai rapidamente sotto del blastoderma, venendo continuamente distesa dal liquido che vi si raccoglie. Ben presto I'allantoide acquista tale sviluppo, sotto del foglio sieroso del blastoderma, da circondare tutto l'embrinne, e nei mammiferi anche la vęscicola ombelicale. I vasi delle pareti dell' allantoide formano numerose anse terminali, le quali penetrano nei villi del chorion sieroso e per questo modo mettonsi in uno stretto rapporto coi vasi della mucosa uterina; cosi succede la for- 
Inizione del chorion villoso vascolare, perchè iu ogui villo $\mathrm{c}$ nelle sue ramificazioni si fanto strada i vasellini provenienti dall'allantoide terminandovi ad anse, ciascuma delle quali ¿̀ costituita da un'arteria e da una vena:

Il chorion vascolare che circonda all'esterno tutto l' novo sí modifica diversamente nei diversi animali :

Placenta diffusa-In alcuni animali come nel cavallo nei marsupiali ed altri i villi si conservano per tutta la vita embrionale.

Cotiledoni-Nei luminanti alcuni villi si atrolizzano ed altri acquistano invece un grande sviluppo, di modo che si formano, per ramificuzione e produzione di nuove anse dalle già esistenti, dei fiocchi vascolari peduncolati sparsi su tutta la superficie del chorion, detti coliledoni, i quali trovansi rinchiusi in corrispondenti sporgenze caliciformi della mucosa dell'utero.

Placenta zonata od a cinto - In altri animali , come nei carnivori, scompaiono i villi ai due poli dell'uovo e si sviluppano invece quelli d'una zona equatoriale, di modo che l'embrionc rimane circondato da una fascia che lo cinge trasversalmente, da cui sporgono superiormente la testa ed inferiormente le natiche.

Placenta a focaccia - Nell' uomo infine, come in altri mammiferi, per esempio nei rosicanti, scompaiono tutti i villi meno pochi, cioè quelli che guardano e toccano direttamente la mucosa dell'utero, e le loro anse terminali sviluppandosi e producendone altre vengono a costituire come un grosso disco formato da tanti lobi o pennelli di anse vascolari che s'intrecciano coi vasi sanguigni della mucosa dell' utero.

FORMAZIONE DELLA MEMBRANA DECIDUA

Quando l' ovicino arriva nell' utero scorre lungo la parete e se venue fecondato si fissa sulla mucosa d'ordinario in vicinanza del fondo, ciò che succede per l'introdursi dei villi 
del chorion primitivo nelle glandule tubulari della mucosa o nelle ferite che si formano per la mestruazione. In questo punto la mucosa si congestiona più che altrove e si tuinelà, come fosse irritata dalla presenza d'un corpo straniero, per circondare l'ovulo con un vallo e quindi con un labbro circolare.Questo è l'inizio della decidua riflessa, che s'innalza intorno all'uovo e sopra dello stesso,finchè si chiude e forma un sacco il quale si distende in proporzione dello sviluppo dell' embrione, acquistando col tempo tale dimensione da riempire tutta la cavità dell' utero. Allora la membrana mucosa del sacco in cui è chiuso l' embrione tocca la mucosa delle pareti dell'utero che più o meno vi aderiscono. Prima però tra l'una e l'altra (decidua riflessa, decidua vera) vi ha uno spazio occupato da un liquido detto perione. Durante questo processo i villi del chorion si son fatti grossi, ramificati, e nel cavo di essi si fecero strada i vasellini dell' allantoide.

La decidua vera e la riflessa saldandosi fra loro formano una sola membrana assai vascolare, che presenta il massimo spessore relativo verso il terzo mese di gestazione; ma in seguito si assottiglia, perde i suoi vasi e si riduce ad una membrana esile che involge all' esterno il sacco amniotico. Nel punto dove si era fissato originariamente l' ovicino la mucosa che tapezza la parete dell' utero, e che si continua colla decidua vera e riflessa, acquista un grande spessore per sviluppo dei proprii vasi sanguigni e costituisce la cosi detta decidua serotina, la quale poi diventa placenta ulerina. Questa porzione della mucosa uterina rimane sempre aderente al tessuto muscolare delle pareti dell'utero, dal quale appunto riceve i suoi vasi sanguigni, che durante la gestazione manifestano una rigogliosa vegetazione e sviluppano tante anse terminali, le quali s'intrecciano con quelle della placenta fetale. 
Sriluppandosi l'embrione alla periferia del blastoderma, pel proprio peso si corica sull'uno dei lati indi s'abbassa infossandosi verso il centro dell' novo. Siccome il foglio sieroso del blastoderma oltre la periferia dell' area germinativa ha acquistato mediante i villi delle salde aderenze col chorion primilivo, succede che l'embrione abbassandosi distende e trasporta seco soltanto quella parte del foglietto esterno del blastoderma che trovasi tra i margini esterni delle lamine parietali c la periferia dell'area opaca; cosi si forma una ripiegatura di questo foglietto tutto all' intorno dell' embrione, la quale mano mano clie l'embrione s'infossa diventa sempre più ampia e forma un vero sacco a doppia parete; questo si chiude infine superiormente in corrispondenza del dorso dell'embrione. Avrenuto il saldamento, la pagina inferiore si stacca dalla superiore la quale rimane in contatto immediato col chorion. Il sacco in cui si è rinchiuso il corpo dell'embrione dicesi amnios ed in principio lo involge toccandolo direttamente; ma a poco a poco si raccoglie un liquido trasparente tra le paretí del sacco ed il corpo dell'embrione, di modo che questo viene a trovarsi sospeso e nuotante nel liquidoche dicesi appunto amniotico.

Negli uccelli il liquido amniotico va aumentando in principio; in seguito viene riassorbito, di modo che verso la fime dell'incubazione il sacco amuiotico rappresenta un velamento che tocea direttamente la superficie del corpo del pulcino.

Nell'uomo invece il sacco dell'amnios contiene sempre una certa quantita di umore; verso il quinto mese raggiunge il maximum, da quest'epoca in avanti diminuisce relativamente senza però scomparire del tutto. Esso serve a difendere il feto dagli urti e dalle scosse che puo ricevere il corpo della madre, c nel momento del parto il sacco amniotico compresso dalle pareti dell'utero, che si contraggono dall'alto in basso, si 
la strada pel primo nel collo dell'utero; e cosi il liquido amniotico raccogliendosi a poco a poco in basso dilata a mo' di cuneo il collo dell'utero preparandolo a dar passaggio al feto, il quale d' ordinario si presenta coll'occipite.

L'amnios sviluppato consta di due strati, l'uno interno od epiteliale, formato da un semplice strato di cellule prarimentose identiche a quelle della superficie del corpo dell' embrione di cui sono una continuazione, cd uno esterno fibroso che è formato da una continuazione immediata della lamina cutanea; per cui alcuni considerano l'amnios come una specie di espansione culanea dell'embrione, la quale staccandosi dalla linea mediana anteriore od addominale, e propriamente dal contorno dell'ombelico, si ripiega all' indictro sulla linea mediana dorsale per formare un sacco che infiue si chiude ermeticamente. Lo strato esterno dell'amnios appartiene istologicamente al tessuto contettivo ; in alcuni animali però contiene anche degli elementi contrattili; esso infatti si contrae e forma delle pliche o rughe alla sua superficic, e spingendo il liquido amniotico in quà ed in là imprime un movimento oscillatorio al corpo dell'embrione, come si può benissimo vedere nei pulcini di gallina del settimo od ottavo giorno d'incubazione. Nelle pareti dell' amnios non si osservarono filora ne' vasi sanguigni ne'nervi.

Il liquore dell'amnios è a!calino; all' epoca del parto è d'ordiliario torbido, perchè tiene in sospensione molte cellule epidermoidali e delle goccioline di sostanza grassa ; per ció clıe riguarda la sua composizione chimica noi lo sappiamo composto di 97 a 99 parti di acqua; il residuo solido conticne albumina, materie grasse, urea, allantoidina, zucchero, sali di acidi organici (lattati ed urati di soda) e di acidi inorganici come solfati, fosfati e cloruri. (1)

(1) Ognuno comprenderd di leggieri che la composizione chimica del liquore amniotico deve variarc coll'età del feto, il quale come può inghiollire l'umorc, puỏ anche versare nel sacco amniotico le suc cserczioni. 
Sebbene tutti i sistemi si abhozzino e si sviluppino quasi contemporaneamente, pure, ad evilare confusioni, crediamo piir opportuno di trattarli l'uno dopo dell'altro cominciando, come è naturale, da quelli che sono i primi a manifestarsi e che più presto acquistano lorme complete e scendendo gradatamente fino a quelli che compaiono e si perfezionano più tardi; così noi incominceremo col sistema nervoso, essendo quello clie rappresenta le prime tracce del corpo embrionale, e finiremo cogli organi genitali, come gli ultimi a comparire od a raggiungere le forme perfette. Per altro saremo obbligati a fare qualche eccezione, per esempio per gli organi dei sensi e specialmente per l'apparecchio vascolare sanguigno, di cui tratteremo affatto in ultimo sebbene le sue parti centrali, come il cuore ed i vasi maggiori, si formino e funzionino fin dai primordî della vita embrionale, e ciò perchè l'apparecchio della circolazione può essere ben studiato e compreso nel suo sviluppo soltanto quando si conoscono tutti gli altri sistemi ed organi.

SVILUPPO DEL SISTEMA NERVOSO

La nola primiliva, come sappiamo, è il primo rudimento embrionale e rappresenta l'asse cerebro-spinale. Noi abbiamo veduto come questa laminetta si trasformava in una doccia, poichè si faceva depressa nella linea mediana, mentre si sollevavano i suoi bordi laterali in modo da formare come due margini o labbri sporgenti verso la membrana vitellina. Mentre questi duc margini laterali sporgenti s'innalzano rendendo piir profonda la doccia mediana, si va aumentando il blastema, sicchè le pareti della doccia si fanno sempre più spesse ed opache. In seguito i due bordi sporgenti piegandosil'un verso l'altro si raggiungono nella linea mediana dorsale e rinchiu. 
dono cosi un canale abbastanza ampio e cilindrico, che rimane tapezzato internamente dal loglietto esterno corneo od cpidermoidale dell'area germinativa, il quale rivestiva superiormente la nota indi la doccia primitiva. Per tal modo l'asse cerebro-spinale a quest' epoca è rappresentato da un canale abbastanza ampio, tapezzato internamente da uno strato epiteliale, mentre all' esterno di questo le pareti del canale sono formate dalla sostanza nervosa allo stato cellulare embrio-plastico appartenente al foglielto mediano. Come vedremo verificarsi per molti altri canali di formazione embrionale, cosi anche in questo si raccoglie un liquido trasparente che va diminuendo relativamente col restringersi del canale stesso. $\mathbf{E}$ a notare per altro che la chiusura della doccia midollare in un canale per formare il canale midollare, non succede contemporaneamente in tutt'i punti, ma piuttosto dall' avanti all' indietro, cioè dalla porzione cefalica alla porzione caudale.

In certi punti rimane aperto fino ad un' epoca avanzata della vita embrionale (seno romboidale o dilatazione caudale) ed in un altro punto (quarto ventricolo o midolla allungata) non si chiude mai, ma viene semplicemente coperto dal cervelletto. Il canale midollare in alcuni animali si chiude quasi interamente in forma di tubo cilindrico, ed in seguito presenta una dilatazione all' estremità anteriore o cefalica ed una all' estrenità posteriore o caudale; in altri invece, come nel cane e probabilmente negli altri animali superiori, $i$ bordi sporgenti della doccia midollare passando ad arco ottuso l'uno nell'altro all'estremità anteriore o cefalica e ad arco acuto alla posteriore o caudale all' epoca del saldamento od anche prima, fanno si che l' asse cerebro-spinale presenti prima di chiudersi una dilatazione sferica all' estremità anteriore ed una romboidale all'estremità candale. Per ciò una volta avvenuta la chiusura il rigonfiamento anteriore rappresenta una vescica sferoidale, mentre il posteriore che rimane per lingo tempo aperto (negli uccelli per tutta la vita) forma un seno ovale o romboidale: quest' ul timo, sia che si chiuda o 
('le rimanga aperto, non mula piì di forma, no acquista un particolare sviluppo.

Camgramenti nolevoli invece si verificano al rigonfiamento interiore o cefalico. il quale la mu notevole e rapido incremento e, per strozzamenti trasversali o circolari, si dividc dapprima iu tre indi in cinque parti o vescicole cornunicanti tra loro; queste da principio si trovano disposte 1: una dietro l'altra in un sol piano, ed in seguito sviluppandosi in uno spazio limitalo (cavilà del cranio primitivo fibroso ) si piegano l'una sull' altra formando tra loro degli angoli, e cosi alcune vengono a ricoprire le altre.

Pare che sicno diverse le cause per le quali le parti dell'asse cercbro-spinale cmbrionale si piegano l'una sull'altra e, formando dei ginoccli od augoli, arrivino perfino a coprirsi viceudevolmente. Non vi ha alcun dubbio che una cagione principalc sta nel precoce sviluppo della porzione anteriore dell'asse cercbro-spinale nella cavilà del cranio primitivo, il cui diametro antero-posterior'c non permetle che le singole parti si sviluppino manteurendosi in serie longitudinale, ma le obbliga a picgarsi per occupare tutta la cavità piuttosto nel senso verticale; pare per altro che anche lo schclctro del cranio si picghi per maggiore cedcrolczza in alcuni punti delle sue pareti; ed è parimenti indubitato che nell'interno stesso della cavilà del cranio per sviluppo di alcune parti, le quali poi cambiano i loro rapporti anatomici, si verifichino delle condizioni che obbligano il cervello a piegarsi.

Per riuscire più facile c compendioso incomincerò dal primo stadio, allorclıc̀ l'estremità cefalica del tubo midollare consta delle tre vescichelte incui si è divisa la vescichetta primitiva per la comparsa di due solchi o strozzamenti trasversali, i quali pero non arrivano mai a cliudere la comunicazione interna tra l' una e l' altra.

Queste tre vesciclette che si distinguouo dall' imrinzi alI' indictro secondo il loro numero d'ordine, cioc prima seconda e ter $\approx a$, riccvellero nomi speciali ; e cosi la prima si 
chiamó prosencefalo, la seconda mesencefalo e la terza epencefalo. In principio, cioè fin verso la quinta setlinama, la vescichetti mediana o mesencefalo è la più voluminosa e quindi la più sporgente. Dalla parte anteriore inferiore de! prosencefalo vedesi presto uscire a mo' di gemma una protuberanza piriforme e cava, la quale nell' uomo e negli animali superiori acquista beı presto grandi dimensioni , talchè impedita di svilupparsi in avanti dalla capsula fibrosa che rappresenta il cranio primitivo, si rivolge in alto ed indietro coprendo, a guisa di un berretto frigio, il prosencefalo da cui ebbe origine; in questo modo l' cstremità cefalica è ora costituita da quattro vesciclıette, di cui la primitiva anteriore è divenuta seconda erl è coperta da quella cui diedc origine : ma contemporaneamente anche nell' epencefalo si è manifestato un solco trasversale che lo ha diviso in due metà, di cui l'anteriore si c̀ piegata sulla posteriore in modo da ricnprirla; la porzione anteriore per inspessimento delle pareti diventa solida, per cui scompare la sua cavità interna, mentre i bordi della metà posteriore sottoposta alla prima si chiudono mai ed invece rimangono divaricati e divergenti allinnanzi formando le pareti laterali del quarto ventricolo.

Ecco così il rigonfiamento cefalico del canale midollare diviso in cinque vescichette, le quali già fin d' ora rappresentano le parti principali dell' encefalo, cioè i grandi emisferi, i talami ottici, i corpi quadrigemelli, il cervelletto ed il midollo allungato. Nei vertebrati inferiori (pesci ed anfibii); la massa encefalica si arresta in questo stadio embrionale di distinzione delle singole sue parti principali ora enumerate, e solo per inspessimento delle pareti si restringono le cavita che rimangono in comunicazione tra loro mediante canali e fessure assai anguste; salendo nella scala degli animali rertebrati noi vediamo sempre più sviluppati i grandi enusferi ed il cervelletto, di modochè già negli nccelli i grandi emisferi acquistano tale sviluppo che si piegano in sopra ed indietro per ricoprire tutte le altre parti, cioe il prosencefalo e 
frarte del nesencelialo, lasciando scoperto il cervellello, che is assai roluminoso e compatto, ed il terzo posteriore del mesencefalo o loli. ollici. Nei mammiferi i grandi emisferi sono cosi sviluppati che coprono anche la porzione anteriore del cervellello, e melluomo infire, raggiungendo il massimo svilappo, ricoprono tulte le altre parti dell' encefalo. Nei vertebrati inferiori il mesencefalo conserva per tulla la vita la sua cavità intcrma e quindi l' aspelto vescicolare, mentre uei vertebrati supcriori, come nell' uomo, le pareti acquislano tale spessore da ridurrc la cavila interna allo stalo di semplice ed angusto canale qual' è l' acquedotlo di Sylvio.

La divisione delle vescicole cerebrali in due melà laterali si verifica per la comparsa d'un solco longitudinale od antero posteriore e la formazione dei due emisferi dipende dallo sviluppo della gran falce, che a modo di un sello si stacca dalla capsula cranica e s'introduce nclla vescicola anteriore dall'alto in basso e dall' avanti all' indietro.

Il tentorio è dapprima verticale ed abbraccia il mescucefalo o corpi quadrigemelli, ma a poco a poco, per lo sviluppo della base del cranio c per l'avanzamento del corpo dello sfenoirle, si meltc nella posizioue orizzontalc fra il cervellello ed i grandi emisferi.

La superficic dei grandi emisferi si mantiene liscia sino al secondo mese; nel corso del terzo mesc le parcti ancor soltili delle vescicole cerebrali antcriori formano tante pieghe, che diconsi circonvoluzioni primitive o transitorie poichc̀ a poco a poco scompaiono di nuovo in modo che nel sesto mesc di geslazione la superficic dei grandi cmisteri è di nuovo liscia, ciò che succede probabilmente per inspessimento omogeneo del blastemanervoso che ne forma le pareti. Nel scllimo e nell' oltavo mese si sviluppanole circonvoluzioni permanenti per escrescenza della sostanza nervosa alla superficie degli cmisferi.

Il inesuncefalo nel sesto mese d̀ diviso in due metà latcrali per un solco longiludinale, e nel setlimo mese vicne diviso in qualtro per un secondo solco trasversale. 
Le celluke embrio-plastiche costituenti le pareti delle vescicole cerebrali e del canale midollare a poco a poco si differenziano, Ic piú inlerne prendono il carattere di cellule epitcliali, le esterne divenlano cellule nervose.

La sostanza midollare o bianca del inidollo spinale si forma per sovrapposizione di blastema a ridosso della sostanza grigia, ed anzi è una produzione di questa. Gran parte della sostanzamidollare bianca del cervello sembra formarsi in sito dalla sostanza grigia, ed in parte è una continuazione dellà sostanza corticale del midollo spinale.

\section{SISTEMA KERVOSO PERIFERICO}

L'ottico c l'olfattorio sono indubbiamente dipendenze delle vescicole cerebrali. E ancora controversa invece la dipendenzal del nervo acustico, che alcuni vorrebbern formato dal peduncolo di una vescichetta dapprima isolata e staccata dal centro nervoso, col quale si metterebbe in rapporto di continuazione dopo un certo tempo.

I gangli ed i cordoni nerrosi periferici si formano in sito da cellule del foglietto mediano dell'area germinativa ed indipendentemente dal sistema nervoso centrale, che puo mancare in tutto od in parte con perfetto sviluppo dei cordoni nervosi, i quali, per un falso giudizio d'analogia si considerano come aventi originc dai centri. Però le vere radici dei nervi motori cercbro-spinali si formano dai centri.

\section{SVILUPPO DELLO SCHELETRO}

\section{- Colonna vertebrale e cranio}

La corda dorsale e le masse subprismatiche laterali alla stessa sono la base dello sclieletro primitivo, come le lamine ai lati esterni delle masse subprismatiche sono i primi rudimenti delle pareti del tronco.

Le masse subprismatiche dapprima sono solide ma in seguito, per fusione delle loro cellule centrali, si forma nel 
mezzo di esse uno spazio cavo, come appunto succede anche uelle lamine o piastrine laterali cle, per lo stesso processo, si dividono in una pagina superiore ed in una inferiore limitauti la cavila pleuro-peritoneale compresa fra la parete del tronco e quella dell' intestino. Dilatandosi in principio la caviti centrale delle masse subprismatiche vengono queste a trasformarsi in lanti sacchi che a poco a poco perdono la loro forma, perchè si adattano colla parele interna al canale midollare, mentre nell'angolo interno inferiore si forma un accumulo di blastema che protende nell'interno del sacco e sempre piì ingrossandosi ne riduce a poco a poco la cavilà. Infine la parete interna, l' inferiore e l' esterna, pel successivo sviluppo di questo blastema, si fondono o meglio si confondono tra loro in un sol corpo solido che rappresenta la inelà laterale dei corpi delle vertebre primitive; invece la parete superiore dei sacchi rimane quasi isolata e costituisce la cosi detla lamina muscolare, perclıe da essa si sviluppano i muscoli della colonna vertcbrale. In seguilo le metà dei corpi delle vertebre di destra si saldano con quelle di sinistra ed abbracciano cosi in basso la corda dorsale e superiormente il canale midollare. Dalla parte delle vertebre primitive che confina, col canale suddetto si stacca una lamina, la quale si fa strada fra il foglio midollare e corneo ed incontrandosi e saldandosi con quella del lato opposto costituisce la cosi delta membrana reuniens superior. La corda dorsalc viene circondata dai corpi delle vertebre dapprima inferiormente. ed ill seguito anche superiormente per un processo dei corpi delle vertebre che s'intromette tra essa ed il canale midollare. Questo blastema che rincliude la corda e che appartiene ai corpi delle vertebre primilive dicesi guaina esterna della corda.Con ciò si lia la formazione dellavera colonna vertebrale primitiva, la quale ne' suoi primordii si presenta allo stato membranaceo. Dalla porzione di questa che involge la corda si formano i corpi delle vertebre, inentre quella parte che abbraccia il canale midollare dà origine agli archi vertebrali o nevro-apofisi. 
Jrima però che queste parti diventino cartilaginee i corpi delle vertebre primitive si scindono trasversalmente in duc meli, di cui l'anteriore dell'una si salda colla posteriore del corpo successivo, e cosi per esempio la prima rertebra toracica permaneritc è formata dalla metà posteriore dellaprima toracica primitiva coll' arco veriebralc corrispondente e dalla metà anteriore della seconda toracica. Tulte le vertebre permanenti si formano in questo modo.

Questo processo di divisione dei corpi primitivi per costituire le vertebre permanenti ha luogo dallinnanzi all'indietro; i singoli pezzetli risultanti dalla fusione si fanno resistenti incominciando a prendere il carattere di vera cartilagine nella parte inferiore mediana più grossa che circonda la corda dorsale. Questa intanto si è fatta compatta ed elastica per aumento dei suoi elementi celluluri, i quali si trovano stipati e stretti in un cordone abbastanza resistente per una guaina membranacca omogenea formatasi intorno ad essi.

In questo periodo le vertebre cartilaginee hanno l' aspetto d' una sella capovolta nella cui concavità riposa il midollo spinale, mentre all' intorno della corda dorsale, per aumento della sostanza cartilaginea, si costituiscono i corpi delle verlebre che stringono all' intorno la corda e la fanno come atrofizzare, per cui perde la sua forma cilindrica e presenta dei rigonfiamenti in corrispondenza degli spazii intervcrtebrali.

Intanto le branche montanti degli archi cartilaginei si incurvano e s'avanzano per raggiungersi nella linea mediana dorsale e cosi completare gli arcli dclle verlebre. La parte della guaina primiliva che rimane tra i corpi delle vertebre costituisce i dischi o leganienti inlerverlebrali, nel centro dei quali si osserva a lungo anche dopo la nascila il rcsiduo della corda dorsale. Invece la parte della membrana reuniens superior che si trova tra gli archi vertebrali forma i legamentigialli od intercrurali. Purò ancoral prima che si chiudano gli archi cartilaginei incomincia l'ossificazione delle vertebre, la qualcè precedu- 
ta da introflessioni clavilormi del pericondrio che servono di guida ai vasi sanguigni che da diversi punti della periferia penetrano nclla sostanza carlilaginea. Le cartilagini quindi cominciano a cambiare aspetlo e colore; dabianco-opalinepellucide si famno giallo-rosec e presentano nelle loro sezioni normali quatho punti di ossificazione, cioè due ai lati della corda e due superiormente nei punti dove si dipartono le branche. I depositi calcarci o di ossificazione si estendono in modo clıc ben presto i due centrali od'inferiori s' incontrano esi fondono in un solo che dapprima comprende la corda dorsale e poi sviluppandosi e comprimendola la distrugge (1). All' epoca della nascita ogni vertebra consta di tre pezzi di ossificazione congiunti o divisi tra loro (secondo il diverso modo di vedere) da poca sostanza intermedia cartilaginca; il pezzo inferiore corrisponde al corpo, i due superiori corrispondono alle due branche dell' ar'co coi processi trasversi e spinosi.

Secondo questo tipo si lor'mano tutte le vertebre mieno poche eccezioni, cioè la prima e la seconda vertebra cervicale e lc coccigee. Il corpo della prima vertebra cervicale si fonde col corpo della seconda e forma l' epistrofeo o processo odontoide, ciò che vien coufermato dal fatto che la corda dorsale passa dal corpo della seconda vertebra nel processo odontoide, e più ancora dai casi scbbenc rari di innata anchilosi o saldamento delle due prime vertelse cervicali. Nelle vertebre coccigee si for-

(1) Parlando della corda dorsale come di altre parti ed organi cmbrionali che"a poco a poco si fanno piccoli e scompajono, usiamo arbitrariamente del le espressioni alrofia e riduzione, le quali non sono reali ma apparenti, cioè simulate dall'arresto di sviluppo o debole incremento di queste parti rispetto alle altre, che sôrte anche dopo pure subiscono un rapido sviluppo. Cosi anche la corda dorsale durante tutta la vita embrionale, va ingrossaudosi continuamente massime negli spazii intervertebrali, ed infatli all'epoca della nascita la porzione di corda dorsale esisteate nello spescore di un logamento intervertebrale è molto piu lunga e grossa dellis corda primitiva, cio che non è conciliabile con un rero processo d'atrolizzazione e nenureno con un arresto reale di srilupeo. 
ma soltanto il corpo e non si sviluppano gli archi. Le ciuque vertebre sacrali rimangono sempre fuse in un sol pezzo.

Ai lati delle vertebre e come dipendenze di queste si formano le coste, dapprima sotto forma di ammassi omogrenei di cellule riunite in laminette, le quali ben presto diventano cartilaginee es'inoltrano uelle piastrine parietali mentre queste si piegano in basso e si avviciuano fra loro nella linea mediana. Allora le priune sette costole si fondono tra di loro cogliestremi anteriori e formano le due melà laterali dello sterno, che ancor esse unendosi nella linea mediana costituiscono una laminelta cartilaginea nella quale ben presto conipariscono diversi punti di ossificazione che si distendouo e s'incontrano reciprocamente; i due estremi (manubrio e processo ensiforme) restano uniti alla porzione mediana per sulura. I casi non molto rari di fissura slerni congenila provano evidentemente questo modo di formazione dello sterno.

L'ossificazione delle coste incomincia da un punto verso la parte dorsale delle medesime e procede quindi verso l'innanzi,ma non arriva mai, anche nell'adulto, a raggiungere lo sterno; soltanto in alcuni vechi si trovano ossificate le carlilagini sterno-costali.

Il cranio primitivo è rappresentato da una borsa membranacea, nella cui parete inferiore (base) si continua il prolumgamento della corda dorsale. Il cranio però non percorre in tutte le sue parti ed in tulta l'estensione delle sue pareti grli stadii di sviluppo pei quali si è formato lo speco vertebrale.

Mentre la vôlta del cranio si ossifica senza passare allo stalo cartilagiueo conservandosi sempre membranacea, la base segue più o meno nel suo sviluppo il tipo vertebrale.

L'illustre Oken fü il primo clie in principio di questo secolo manifestó l' opinioue che la base del crauio nou fosse altro clie la porzione anteriore della colonua vertebrale modificata. La leoria diOken fuaccettata come inoppugnabile dal punto di vista generico, però gli embriologi non sono ancora ben d'accordo intorno alla comparazione delle ossa alla base del cranio e 
lor'o parti colle vertebre c loro processi; come pure non si è ancora stabilito un'accordo sul numero delle vertebre dalle quali si fa sviluppare la base del crinio: c cosi vi ha chi la consider'a costiluita da due, chi da tre, chi da quattro e persino da cinque vertebre. Comunque sia, vi hanno almeno due ossa alla base del cranio nelle quali il tipo vertebrale si conservaevidente anche nell' adulto; nell' embrione poi in certi stadii vi ha maggior somiglianza tra queste ossa e le vertebre, che non tra alcune vertebre permanenti come per esempio tra le cervicali e le coccigee.

Esprimendo i nostri dubbii sulla natura vertebrale dell'osso clmoide, considerato da taluni come prima veitebra cranica, ammeltiamo senza alcun commento che l'osso sfenoide in principio è rappresentato da due parti che corispondono a due vertebre, poichè il corpo dello stesso è diviso in due metà (anteriore e posteriore) ciascuna delle quali è fornita di un corpo e di nevro-apofisi od archi vertebrali. La metà anterioresarebbe il corpo della prima vertebra cranica, mentre le piccole ali rappresenterebbero le nevro-apofisi corrispondenti ; la metà posteriore del corpo dello sfenoide e le grandi ali corrisponderebbero alla seconda vertebra cranica. Le nevro-apofisi di queste vertebre non si raggiungono nella linea mediana superiore e perció non arrivano mai per se stesse a chiudersi superiornente. Il corpo della terza verlebra ¿̀ rappresentato dall' osso basilare, le sue nevro-apofisi formano i condili e la porzione inferiore della squamma dell' occipitale che passa per lo stato cartilagineo.

Che veramente queste due ossa, le quali in origine erano tre, appartengano al tipo vertebrale lo prova non solo la forma, massime nei primordii del loro sviluppo, ma più ancora il Callo che nei bruti la corda dorsale si trova nella linea mediana degli stessi e precisamente nel centro di quella parte che rappresenta il corpo delle vertebre, cioè in lutto l'osso basilare e nel corpo dello slenoide.

"Nei feli umani abbiamo osservalo che la corda dorsale ter- 
mina quasi all' apice del processo odontoide e nessuna traccia della medesima si rinviene nelle cartilagini corrispondenti all'osso basilare ed al corpo dello sfenoide. A conciliare questo fatto con quello che si osserva nci feti dei bruti giova considerare che la corda dorsale c (Ved. Fig. A. B. C. D.) è nell'embrione l'asse di sostegno del corpo ed ad un tempo il mezao d'unione fra la doccia superiore midollare t. m. e la doccia inferiore intestinale $t$. $i$. che nei primi momenti di loro formazione coincidono coi loro estremi, (Ved. fig. B. ); ma la corda dorsale c, come si disse nella nota a pag. 87 , non ha quel rapido sviluppo delle parti che sorgono in seguito intorno ad essa, e perciò netl'embrione umano la corda dorsale rimane indietro col tubo inferiore od intestinale, mentre il tubo midollare si sviluppa assai precocemente all innanzi col suo rigonfamento cefalico. $(V e d . f g . A$ e $)$. L'opposto succede nei bruli, nei quali il rigonfiamento cefalico s' arresta indietro colla corda, mentre il tubo inlestinale si sviluppa all'innanzi. (Vedi Fig. B e D.) (1).

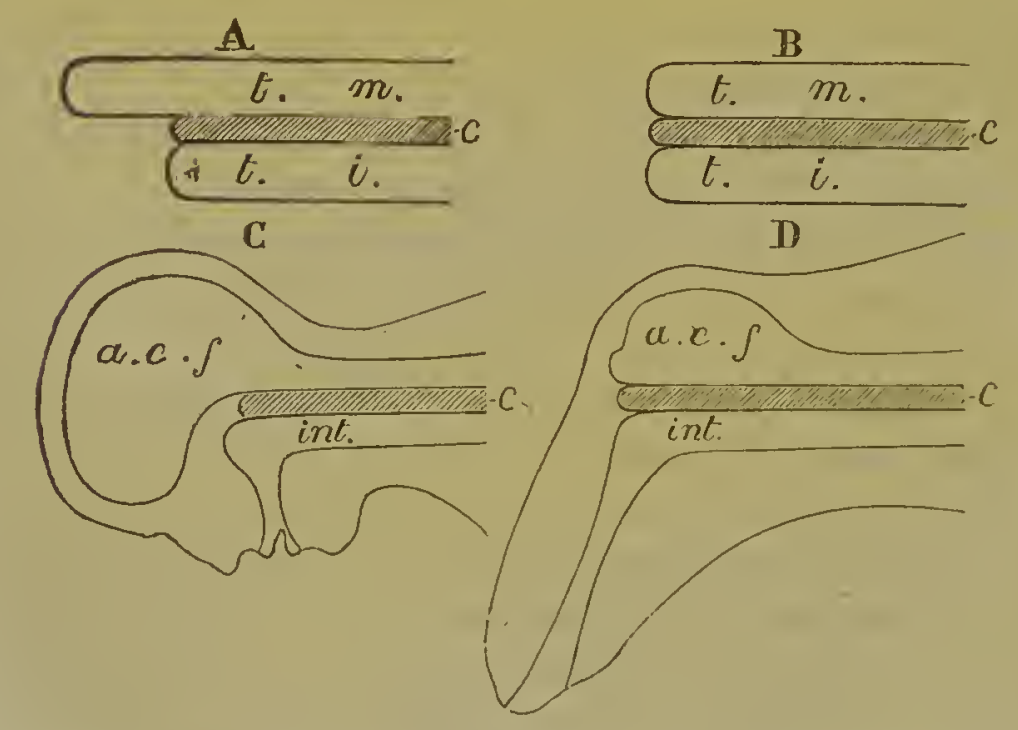

(1) Vedi rendiconto della R. Accademia della scienze fisiche e matemaliche di Napoli fase. 9. e 10. Setl. e Otl. 1867. Sulla natura detle ussa alla base del eranio. Sibiui e Renzone. 
Il resto della capsula cranica si fa ossea senza passare allo stato di cartilagine. La reuniens superior delle tre o qualtro vertebre craniche forma un'ampia capsula membranacea nella quale si sviluppano dei punti di ossificazione, cle a modo di isolette a contorni dentellali si dilatano e s'incontrano vicendevolmente. Di questi punti di ossificazione se $\mathbf{n}$ osservano due (cioc uno a destra, l'altro a sinistra) nella regione frontale ed occipitale ed uno per ogni regione parietale.

La porzione petrosa dell'osso temporale non appartiene veramente al tipo cranico, ma può essere considerata come un osso involgente un organo di senso (udito). Essa è primitivamente cartilaginea e viene imprigionata in una piega della capsula fibrosa involgente la massa encefalica, la quale capsula fornirebbe la parte squamosa del temporale per un punto di ossificazione che distendendosi s'incontra superiormente coi punti di ossificazione del parietale ed occipitale, inferiormente colla porzione petrosa, colla quale si salda, ed anteriormente colle nevro-apofisi della seconda vertebra cranica, ciò̀ colle grandi ali dello sfenoide.

Siccome all'epoca della nascita i punti di ossificazione non sono ancora giunti ad incontrarsi completamente, cosi rimangono tra un'osso e l'altro degli spazii ancora occupali dalla capsula fibrosa che diconsi fontanelle (1).

\section{B) Sviluppo della faccia}

La porzione cefalica od anteriore del canale midollare sviluppandosi precocemente sporge oltre l' area vascolare, per cui si forma una piega tra la capsula celalica e l'area germinativa, la quale ruppresenta una specic d'imbulo aperto allo-

(1) Fissando l'occhio od applicando la mano con delicatezza su queste regrioni nei bambini si avvertouo le pulsazioni del cervello sincronicle ai movimenti del cuore c della respirazione, movimenti che ricscono piu mareali quaudo il bambino piauge o grida. 
innanzi e sottoposto alla massa encefalicaclie si è un poco incurvata. Quest'imbuto comprende il tralto d'ingestione del tubo intestinale, e termina all' indietro a fondo cieco; le sue pareli rappresentano la regione facciale e del collo dell' embrione.

Le pareti laterali di questo imbuto sono coperte all'esterno dal foglietto corneo, ed il foglietto motorio-germinativo sottoposto non si divide, come al tronco in due pagine, per cui le pareti del tubo d'ingestione rimangono aderenti alle pareti del collo.

Per raccolta di blastema la regione cervicale diventa molto grossa. quasi cilindrica e sporgente. Fra la terza e la quarta seltimana nelle pareli inspessite si presenta dapprima un solco nella linea mediana, ed in seguito tre o quattro solchi trasversali direlli dall' avanti all' indietro verso la colonna vertebrale. Questi solchi facendosi profondi diventano vere fessure, ed allora diconsi fessure branchiali, mentre le piastrine del blastema interposto, che naturalmente sono piegate ad arco, diconsi lamine od archi branchiali o viscerali, perchè corrispondono perfettamente agli archi branchíali che si osservano per tulta la vita nei pesci; infatti come in questi animali così anche nell' embrione degli uccelli, dei mammiferi e dell' uomo si portano a queste parti i rami principali piegati ad arco della grande arteria che esce dal cuore.

Il primo arco branchiale o superiore trovasi fra l'apertura superiore dell'imbuto e la prima fessura branchiale; limita pertanto in basso ed ai lati quell' enorme apertura informe soltoposta alla regione froutale, che rappresenta l'adito orale emhriornale e che mena in una cavità poco profonda, la di cui parete superiore, come abbiamo veduto, è formata dalla base del cranio primitivo.

Questo primo arco costituisce il processo di Meckel intorno al quale si deposita il blastema che diventa cartilagineo poi osseo e cosi si forma la mandibola. All' estremità posteriore di questo primo arco si osserra un prucesso, il quale 
sviluppandosi all'aranti verso la linea mediana ed incontrandosi con due processi o protuberanze dirette in basso e provenienti dalla capsula fibrosa o cranica, viene a formare la cavità dell' orbita.

Questi clue processi del primo arco brancliale portandosi sempre più innanzi incontrano l'estremità incurvata corrispondente e diretta allesterno di un terzo processo biforcato mediano che si origina dalla regione frontale della capsula cranica. Per tal modo si forma la mascella superiore, la quale s'interpoue tra la base del cranio e la cavità orale.

Ognuno ora comprende facilmente come in principio le aperture nasali si trovino assai lontane fra loro e restino divise dalli cavità orale soltanto all'esterno, mentre all'interwo sono in aperta comunicazione. La divisione fra le fosse nasali elá cavità orale si fa sempre più profonda per lo sviluppo delle osse palatine alla faccia interua del processo del primo arco branchiale. L'estremita posteriore del primo arco viene imprigionata nel blastema, da cui si origina la parte petrosa dell'osso ternporale, e costituisce l'incudine ed il martello; la estremità posteriore della fessura branchiale seconda, ciò̀ quella che sta tra il primo ed il secondo arco si allarga e rimane sempre aperta costituendo la fessura uditiva; la staffa, il processo stiloile, il ligamento stiloideo ed il piccolo cornó dell'osso ioide si formano dal secondo arco branchiale; il terzo arco branchiale costituisce il corpo ed i grossi corni dell'ioide. Il quar to le cartilagini laringee.

Nella quinta settimana tutte le fessure sono chiuse meno quelle che hanno costituite le aperture permanenti, cioè le orbite, le narici, la bocca ed il meato uditivo esterno.

\section{C) Suiluppo degli arti}

Le estremitá compajono verso la quartu settimana sotto forma di quattro bottoncini ai lati del corpo, ma in maggior vicinanza della linea mediana dorsale. Dapprima si veggono 
le anteriori, indi le posteriori ; constano di blastema appartenente al foglietto mediano ricoperto dal foglietto esterno a corneo epidermoidale. Nel corso della quinta seltimana questi botloncini si allungano e mediante strozzamenti trasversali si presentano divisi in due meta, una posteriore cilindrica ed una anteriore schiacciata concavo-ronvessa, in modo che si adatta come una palelta alla parete del tronco (mano); ben presto si presentano in questa quattro solclii longitudinali che segnano i primi spazii interrigitali.

Nell' ollava setlimana la porzione cilindrica posteriore per un leggiero angolo si distingue in due parti, braccio ed antibraccio, coscia e gamba; a quest' epoca ancora non vi ha differenza di forma fra l' arto superiore e l' inferiore. I\} cinto omerale e pelvico debbono considerarsi come dipendenze del tipo vertebrale che si mettono in rapporto cogli arti, il blastema dei quali appartiene assai probabilmente alle lamine parietali.

Il processo di ossificazione non s'inizia contemporaneamente in tutte le ossa, cosi per esempio la clavicola incomincia ad ossificarsi fra la settima ed ottava settimana, mentre le epifisi delle ossa cilindriche e le piccole ossa del carpo e del tarso, come la base della scapula, l' acromio ed il processo coracoide si ossificano dopo la nascita.

SVILOPPO DEL TUBO GASTRO-ENTERICO ED ORGANI ANNESSI

Noi abbiamo veduto formarsi la porzione anteriore del tubo gastro-enterico da una ripiegatura dell'area germinativa conformata ad imbuto e giacente al di sotto della capsula del cranio. Quanto avviene anteriormente succede pure, sebbene in grado minore, anche all' estremità caudale per l'allungarsi dell' asse cerebro-spinale e della colonna vertwbale, che si solleva dall' area germinativa e si fa sporgente all' indietro; per tal modo vediamo formate le due aperture naturali del tubo gastro-cnterico, le quali non comunicano col tubo stesso, 
perches mellono in cavila poco profonde terminanti a fondo cieco. Fril gucsti due estremi, che diremo d'ingestione e di egestione (clie dal primo si formano la bocca le fauci e la fidringe, e dil secondo l'intestino retto ) si sviluppa la porzione intermedia. L intestino intermedio è rappresentato, come gii sappiamo, dalla cavita primitiva del corpo che ha la figura dell' interno d' una pianella, tapezzata dal foglietto interno o mucoso intestinale; anche questa porzione inter= media termina come sappiamo tanto all'avanti come indietro a fondo cieco, e per l' allumgarsi succescivo dell' intestino giunge a mettersi in contatto coi fondi ciecli dell' intestino cefalico e caudale.

Nel descrivere la formazione della vescicola ombelicale abbiamo veduto come i margini della porzione intermedia del tubo intestinale si continuano nell'area germirativa, la quale si é fatta intanto vascolare e trovasi a ricoprire un segmento della vescicola ombelicale. Avvicinandosi sempre più reciprocamente $\mathrm{i}$ bordi dell' intestino e delle parti del corpo, l' intestino. intermedio acquista la forma di nn cilindro cavo terminante sempre a fondo cieco ai due estremi, e comunicante circa a metà della sua lunghezza colla vescico'a vitulina. Finalmente le pareti dell'intestino circondate dalle pareti addominali si chindono nella linea mediana, lasciando nel mezzo un' apertura pel passaggio del peduncolo della vescicola vitulina o dollo omfalo-mesenterico, le cui pareti si continuano con quelle dell' intestino, di modo che al di sotto del foglietto corneo epidermoidale (clie dalle pareti del corpo passa a vestire il dotlo della vescicola ombelicale) esiste una fessura circolare che mena nella cavità pleuro-peritoneale. Il dolto omfalomesenterico è cavo e stabilisce la comunicuzione direlta fra la cavilà dell'intestino e quella della vescicola ombelicale.

L' intestino primitivo resta cosi formato dal foglietto mucoso intestinale e dalla pagina interna del loglielto mediano; esso occupal tulta la cavita toracica ed addominale, rimanendo peris diviso dalle pareti del corno per la cavita pleuro-perito- 
neale formatasi frala pagina interna e l'esterna del foglielto mediano: soltanto nella linea mediana dorsale non essendosi formata la divisiove del foglietto mediano nelle due pagine, l'intestino vien tenuto in sito contro la colonna vertebrale primitiva. Il blastema di saldamento fra l'intestino e Ia colonna vertebrale rappresenta il mesenterio primilivo. Il tubo gilstro-enterico, che in principio era più o meno cilindrico e rettilineo, si allunga e perciò si piega ad ansa sporgente, dalla cui sommili si vede partire il dotto omfalo-mesenterico. F cosi si ha già una divisione dell'intestino intermedio in una branca superiore ed in una inferiore; la prima com`è naturale, allungandosi verso l'estremilà cefalica dovrà metlersi in comunicazione coll'apertura d'ingestione e l' inferiore coll' apertura di egestione.

La branca supcriore si dilata in alto e costituisce il primitivo ventricolc fusiforme che in principio è disposto rerticalmente, mentre la branca inferiore presenta in alto un rigonfiamento, intestino cieco, dal quale si vede poi sorgere l' appendice vermiforme. Non sarà difficile ora l' intendere come pel cliudersi delle pareti addominali, l' ansa intestinale dapprima sporgente, ritirandosi nella cavità addominale, eseguisca um mezza rotazione intorno a sè stessa, in modo da formare un cappio, di cui la branca sottoposta rappresenta l'estremità inferiore dell'intestino tenue, mentre quella che vi sta sopra è il principio dell' intestino grosso, cioè la porzione rigonfiata o cieca che si continua ncl colon trasverso e discendente. Il colon ascendente si sviluppa molto tardi e continua ad allungarsi dopo la nascita per l'abbassarsi del cieco verso l'osso ileo, cioè per l' allungarsi dell'angolo destro del colon trasverso. Infatti nel bambino appena nato non esiste quași ancora il colon ascendente ed il cieco trovasi molto in alto solto del lobo destro del fegato.

Il tratto della branca superiore dell'ansa primimitiva, compreso tra il ventricolo fusiforme ed il cieco, si sviluppa molto in Iunghezza e, stante lo stretto spazio in cui è compreso, forma le diverse anse del paccheulo intestinale tenue. 
Il ventricolo si clilata gradatamente mentre prende una posizione piutlos to orizzontale o trasversale, ed allontanandosi dalla colonna vertebrale esegue anche una rotazione intoruo al suo asse longitudinale in modo che il suo margine sinistro diventa anteriore ed inferiore; e perciò il blastema clıe ló teneva legato alla colonna vertebrale si distende a modo di membrana e forma il meso-gastrio, mentre a poco a poco l'estremità sinistra del ventricolo si rigonfia per formarne il londo cieco.

Il principio dell'intestino tenue, cioè la parte clıe vieu dopo del ventricolo, si mantiene in sito; in seguito alle rotazioni del ventricolo ed allo sviluppo del digiuno subisce diverse inflessioni e cosi si origina l'ansa duodenale a ferro di cavallo.

Intanto l' intestino intermedio ha raggiunto coi suoi due fondi ciechi quelli dei tratti d'ingestione e di egestione, $\mathrm{i}$ quali alla loro volta si sono fatti più profondi. In seguito poi al saldamento di questi tratti e per corrosione delle pareti intermedie ai fondi, si stabilisce la comunicazione fra le tre parti in cui il tubo gastro-enterico era diviso primitivamente. L' esofago sembra che si formi per l'allungarsi del tratto di saldamento delle pareti dell' imbuto d' ingestione con quelle del fondo cieco superiore dell'intestino al di sopra dello stomaco.

INNGUA, DENTI, GLANDUIE SALIVARI E MUCIPARE

La lingua appare sotto la forma di una piccola papilla nella linea mediana della faccia interna del primo arco brancliale o mandibula. Questa papilla s' ingrossa e si allarga acquistando a poco a poco la sua forma naturale, e si la sporgente oltre il margine della mascella inferiore; indi si arresta relativamente nel suo sviluppo e viene ad essere ritirala a poco a poco nella bocca, la quale si cliude per lo sviluppo delle mascelle e delle lablıra. Le papille coniche e circunvallate appaiono nel ter\%o mese, cioè poco tempo dopo che si svilup- 
parono ai margini della mascella superiore ed inferiore i solchi dentarii, i quali sono riresliti della mucosa orale sulla cui superficie si reggono comparire delle papille libere in numero di dieci per ogni mascella. Le papille vengono in seguito a chiudersi in sacchetti, poichè ai margini delle mascelle la membrana mucosa forma due rialzi, i quali si avvicinano tra una papilla e l'altra, si saldano tra loro e si chiudono superiormente.

" E cosi, come si vede, i denti sono produzioni della mucosa e uon sono dipendenze del sistema osseo. I germi della sostanza.eburnea sono rappresenati dal conneltivo della mucosa che s'iunalza e forma le papille dentarie; all'incontro la sostanza adamantina della corona è l'origine epiteliale, perchè viene ad essere formata da un ammasso a modo di clavo o coperchiello di cellule epiceliali (in corrispondenza dell'apice della papilla) il quale si sviluppa dall'alıo in basso, cioè s'innoltra verso la base delia papilla sporgente dal connetlivo.

Non si conoscono ancoro i primordii delle glandule salivari, di cui trovansi descritti soltanto gli stadi avanzali di formazione, cioc̀ quando hanuo già l'aspetto di dotti racemosi contencruti cellule in piu strati, che ne riempiono piú o me110 il lume, e di tessuto fibroso di conmeltio involgente in cui si ramificano i vasi sanguigni ed i nervi. L'incremento di quest.e glandule succede per gemmazione alle estremità dei dotti e delle loro ramificazioni. Il lume dei dotti incomincia a Cormarsi dal doito escretore principale e procede verso la periferia delle glandule, finchè diventano cavi anche i rigonfiamenti terminali od acini.

Nella seconda metà del secondo mese di gestazione si refono già iniziate le tre glanılule salirari; e, sc rogliamo prestar fede agli autori, la prima a comparire sarebbe la sottoinascellare e l' ultima la parotide.

(Kölliker è d' avviso che la furmazinue di quesse glandule incominci da una proliferazione dell' epicelio orale, il quale s'innolıa rıci tessuti esterni e si circonda diconneltivo). 
Le glanclule muccipare orali si formano molto piu tardi, cioè nel quarto mese, e sono in origine, come tulte le altre glandule, produzioni solide degli strati profondi epiteliali e quindi ilel foglintlo muroso.

\section{FE(IT)}

Il fegato è una glandula che presto si sviluppa, anzi d' ordinario appare contemporarreamente ai corpi del Woll, e quindi dobbiamo ritenere che quest'orgaro abbia al pari dei reni primitivi una grande importanza pei processi vegetalivi dell'embrione. I rudimenti del fegato si distinguoro ancora prima che nei corpi del Wolf si formino gli acini.

L' origine del fegato è la seguente; lateralmente alla porzione duodenale del tubo gastro-enterico si depone del blastema in forma di due ammassi o lobi, nei quali ben presto si osserva una cavità che comunica con quella dell' intestino mediante due brevi canali, che a modo di appendici cicche si svilupparono lateralmente all' intestino stesso ; il tessuto fibroso dell' intestino formato dalla pagina interna del foglictto mediano si prolunga su questi ammassi, i quali per tal modo s'ingrossano assai e costituiscono i due lobi del fegato. 1 grossi vasi omfalo-meseraici che entrano nella cavità addominale per portarsi al cuore vengono ad essere circondati da questi lobi, ai quali danno molti rami, per cui anche per questo lo sviluppo e la finzione dell'organo assumono in questo periodo della vita embrionale una grande importanza. Per la presenza dei molti vasi sanguigni che si ramificano nel legato, quest' organo fin dalla sua origine è intensamente colorato.

Verso il terzo o quarto mese il fegato è tanto sviluppato da occupare più di due terzi della cavità addominale e da ricoprire quasi tulto il pacclielto terue e l' ansa del grosso. Verso il quinlo mese però si arresla (relativamente) nel suo sviluppo, e si stabiliscono frer tal morlo a poco a poco, come ve- 
dremo per altri organi, le proporzioni di peso e volume tra i diversi organi splaucnici, processo che si continua anche Jopo la nascita e raggiunge il vero complemento nell' età adulta (1).

II pancreas si sviluppa quasi contemporaneamente al fegato; esso al principio compare sotto la forma di un' appendice della faccia interna dell' ansa duodenale, ed è quindi costituito all' interno da un ammasso di cellule epiteliali erivestito all'esterno dallo strato fibroso.

In alcunianimali, come in certi pesci, se ne vedono comparire moltissimi, che si allungano e conservano la forma cilindrica terminante a fondo cieco (appendici piloriche).

Le altre glandule intestinali, come si sa dall anatomia, giacciono nello spessore della parete del tubo digerente. Esse si originano dal foglio cellulare interno, il quale forma tante extroflessioni o meglio dei clavi solidi, i quali si fanno strada nella pagina interna del foglietto mediano che circonla l' intestino; e perciò le glandule parietali del tubo gastroenterico sono rappresentate in principio da clavi filiformi semplici o ramificati del foglietto mucoso intestinale, circondati (la uno strato esterno fibroso di connettivo e di fibre muscolari disposti l'uno presso l'altro a palizzata. A poco a poco per fusione delle cellule interne, si fanno cavi . ed allora si hanno dei tubi tapezzati internamente dalla continuazione dell'epitelio intestinale (cripte, glandulc mucipare e pcpto-gastriche).

(1) Il peso del legato nel neouato sla a quello del corpocome 1. 18, mente nell' adulto í di $1: 36$ 
PEITTONLO

In tutti i libri d'anatomia troviamo descritto il peritonco come un sacco chiuso dal quale sono involli i visceri addominali, mentre nessuno è contenuto nella sua cavità, eccetto le estremità aperte delle trombe faloppiane uella donna. Per tale descrizione si sarebbe indotti a pensare che la cavità addominale del feto fosse primamente occupata da un sacco le cui pareti verrebbero introflesse dai visceri clıe si sviluppano $\mathrm{c}$ che vi s'involgono. Niente di tutto questo, ed il paragone usato dai maestri d'anatomia descrittiva serve soltanto a rendere facile allo studente il comprendere la continuità del foglio sieroso viscerale, che copre all' esterno i visceri addominali, col foglio sieroso parietale, che tapezza all'interno la parete addominale.

Il peritoneo non è altro che il primo strato della pagina viscerale e parietale del foglietto mediano, il quale limita la cavilà pleuro-peritoneale; e perciò resta il più esteruo delle pareli dell' intestino e dei visceri addominali ed il più interno delle pareti addominali.

La superficie interna della cavità pleuro-peritoneale diventa liscia e levigata rimanendo coperta tanto sulle pareti addominali che sugli organi contenuti in quesla cavità da urı strato di cellule. In corrispordenza della colonna vertebrale la pagina parietale passa nella viscerale e quando l' intestino si allunga e si stacca dalla colonna vertebrale, tira dietro di se il. blastema che, seguendo l'iutestino nel suo sviluppo, si assottiglia, diventa Iaminare e forma il mesentere.

E questo vien provato dal fatto anatomico conosciuto da tutti, cioè che gli organi addominali ed i tratli dell'intestino, i quali nell'nomo rimangono ill sito senza staccarsi dalla parete addominale (come il pancreas, i reni, l's iliaca, poruione del duodeno), vengono coperti dal peritoneo soltanto anteriormente, poichè questa membrana liscia dalla perife- 
ria degli organi suddelli passa immedialamente- a rivestire le pareti addominali.

Veduto il processo generale di formazione del peritoneo ci sarà anche facile di comprendere lo sviluppo di alcune sue appendici o parti, quali sarebbero il grande ed il piccolo omento. Siccome il ventricolo primilivo fusiforme e verticale, muta gradatamente di forma, si stacca un poco dalla colonna verlebrale. e nel disporsi orizzontalmente gira intorno al suo asse maggiore in modo che il suo lato sinistro diventa la grande curvatura sporgente in avanti ed in basso, ed il lato destro forma la piccola curvatura rivolta in alto ed indielro, ne risulta dietro dello stesso un vuoto, limitato superiormente ed inferiormente da lamine del meso-gastrio, che si staccano dalgrande e piccola curvalura del ventricolo.

Il ineso-gastrio inferiore va dalla graude curvatura alla linea mediana posteriore delle pareli addominali; quello della piccola curvalura passa a modo di ponte sul fegato, e pertanto to spazio retro-ventricolare comunica colla cavità peritoneale inediante una fessura esistente dietro della porzione pilorica alla piccola curvalura, forame del Winslow.

Il meso-gastrio inferiore o della grande curvatura già nel secondo mese di gestazione ha acquistato tale eslensione da formare una plica libera e pendente dalla laccia anteriore del ventricolo; da questa appunto si origina il grande omento, che gradatamente giunge a coprire dal davanti il colon trasverso; in principio il grande omento è affalto libero, ma in seguito la sua lamina posteriore acquista aderenza colla lamina superiore del meso-colon, e discendendo sempre più copre a guisa di grembiale il pacchetlo intestinale.

Il piccolo omento si forma analogamente da una duplicatura del meso-gaslrio superiore. 
La miläa si sviluppa verso il secondo mese nelmesn-gasirio sinistro, iu gran riciuanza del veutricolo da un blastema che appartiene alla pagina iuterua del foglietto mediano. II suo sviIuppo è Iento; iu principio constia soltanto di piccole cellule; nel terzo mese incomincia a farsi vascolare e pare che $i$ corpuscoli del Malpighi si manifestino soltanto verso lia fine della vita fetale.

Le glandule linfaliche mesenterialisi sviluppano secondo Sertoli nel modo seguente :

In principio si osservano tra le lamine del mesenterio e lateralmente ai vasi sauguigni degli spazi vunli irregolari, più o mello comunicanli tra loro e dordinario disposti longitudinalmente dall'intestino alla radice del mesenterio; in seguito si verifica un accumulo di nuclei (?) intorno a questi spazii. che iutanto si allungano, si piegano e si avvolgono su di loro stessi: da ciò viene chu il mesenterio in corrispondeuza di questi rudimenti di gangli linfatici presenta delle sporgenze opache. Continuando lo sviluppo e l'accumulo dei nuclei all'estremila intestinale del ganglio primitivo, gli spazi o rasi linfatici rengono spinti all'estremo opposto intorno ai vasi sanguigni. Questa parte depressa corrisponde al futuro ilo del ganglio, e l'altra piu sporgente e tondeggiante rappresenta la sostanza corticale, il cui strato piu esterno diventa fibroso c manda sepimenti nell'interno iniziandone la divisione in follicoli.

SVILUPPO DEI POTMONI E GLANDUIE ANNESS:

Il polmone si sviluppa presto tanto nel pulcino quanto nei mammiferi, però sempre piu tardi del fegato. Ecco la descrizione dello sriluppo del polmone rataci dal Baer. Alla porzione inferiore dell' esofago si appalesano due piccole appendici laterali, le quali ben presto prendono l'aspelto di due sacchel- 
li pirilormi, che approssimandosi fra loro verso la linea mediana si forulono in modo da rappresentare un solo sacco diviso in basso iu due melà laterali. La porzione superiore semplice si allunga, assumendo la forma d'un tubo cilindrico (trachea) mentre i sacchetli (polmoni primitivi) si dilatano e si allungano sviluppandosi in basso. Reirlıert e Bisclioff negarono questo modu di origine del polmone, ma Remak ha dimostrato coi suoi ultimi lavori che le cose erano veramente quali le avea descritte Baer.

11 polmone studiato da Coste in un embrione umano, fra il venticinquesimo e ventoltesimo giorno di gestazione, si mostrava formato da due sacchetti piriformi con una cavita semplice, i quali superiormente terminavano in un breve canale mediante il quale comunicavano coll' esofago. La parete de' polmoni in questo stadio era formata di due strati, cellulare interno e fibroso esterno.

L'ulteriore sviluppo del polmone è simile a que!lo delle glandule acinose, poichè lo strato cellulare produce come delle gemme, le quali danno origine ad altre. Lo strato esterno segue le gemme nel loro sviluppo; ed in tal modo tutto il polmone primitivo rappresentato da un semplice sacco si trasforma in un albero di canaletti cavi terminanti con fondi ciechi rigonfiati .

Le pleure si sviluppano intorno al polmone come il peritoneo intorno all intestino, cioè dalla superficie esterna della pagina viscerale del foglietto mediano, la quale in vicinanza della colonna vertebrale si continua sulla faccia interna della pagina del foglietto stesso che forma le pareti della cavita toracica.

La divisione della cavità toracica dall'addominale per mezzo del diaframma si compie quando le due metà dello sterno si saldano tra loro chiudendo la cassa toracica nella limea mediana anteriore. Questo muscolo sembra una produzione della faccia interna delle coste inferiori di ciascun lato, che si avanza nella linea mediana ed ivi si chiude inccutrandosi con quella dell'altro lato. 
Ial glandula tiroidea probabilmente si sviluppa nell' uomo come nel pulcino da un inspessimento dell'epitelio delle fauci, che si appalesa sotto la forma di una macchielta circolare bianca, situata uel blastema cle riunisce anteriormente a modo di membrana gli archi branchiali ed immediatamente al di sopra dell'origine dell'arteria aorta dal cuore.Questa parte di epitelio forma quindi una sporgenza saccata; la quale si riveste di uno strato fibroso del foglietto mediano e finalmente questo strato stringendosi intorno alla base dell'ammasso cellulare, lo stacca dal punto d'origine, mantenendolo però fisso in corrispondenza della biforcazione dell'aorta primitiva. Nei rettili e negli anfibi la glandula tiroidea si trova infatti alla biforcazione dell'aorta primitiva anche negli animali adulti; mentre nei mammiferi già durante la vita fetale, per l'abbassamento che va ad eseguire l'aorta primitiva, la tiroidea resta in alto in corrispondenza della trachea.

In questi animali superiorila tiroidea primitiva si divide per un solco mediano in due metà laterali, le quali hanno l'aspetto di due vesciche cave situate ai lati della trachea ed alla faccia interna della carotide. Dilatandosi sempre più queste vescicole si formano alla loro superficic nuovi strozzamenti visibili anche allo esterno, i quali sono le tracce dei lobi della glandula; verso la fine del periodo fetale, i. singoli lobi si isolano gli uni dagli altri. Contemporaneamente a queste modificazioni esterne si formano dall'epitelio che tapezza internamente la vescica, residuo dell' epitelio delle faucji, delle escrescenze solide, le quali per strozzamenti si dividono e per fusione delle cellule interne si fanno cave.

TIMO

L'origine primiliva di quest'organo è ancora oscura, pare per altro quasi certo, che esso venga a formarsi dal foglietto mediano e precisamente dalla pagina fibrosa dello stesso che involge il tubo d'ingestione. Secondo Bischofr, il 
quale la descrive negli embrioni dei ruminanti, questa glandula si presenta solto la forma di due delicate strisce di blastema, disposte l' una appresso dell' altra al daranti della trachea, le quali si foudono superiormente col blastema della tiroidea. Secondo le ricerche di Simon questi rudimenti della glandula timo sarebbero formati da tubi di una membrana anista, la quale presenterebbe quà e là degli ingrossamenti fusiformi ; nello interno di questi lubi si trova una massa granulare ed un certo numero di nuclei e, secondo Kölliker, di cellule nucleari. I tubi primitivi presentano in seguito delle sinuosità laterali, le quali sviluppandosi e dividendosi formerebbero cosi i lobi della glandula.

I reni permanenti si sviluppano come i polmoni solto forma di due appendici a modo di extroflessioni laterali della parete della cloaca, vale a dire della porzione inferiore dell' intestino che si è dilatala e sta in comunicazione con quella parte dell'allantoide, la quale è rimasta rinchiusa nella cavità addominale e rappresenta la vescica urinaria primitiva. Le ripiegature sorgono precisamente al confine fra l'allantoide e l'intestino ; esse in principio sono cave e semplici, le loro pareti constano dei medesimi strati delle pareti della cloaca, cioè dello strato cellulare all'interno e del fibroso all'esterno. In seguito si sviluppano portandosi in alto ed ai lati della colonna vertebrale, ed in questo modo si formano gli ureteri, i quali alla loro estremità superiore si allargano e presentano tante sinuosità, cioè i rudimenti dei calici renali. Per inspessimento dello strato fibroso intorno a questi si origina il corpo glandulare compatto del rene permanente. Una volta clie l'organo ha raggiunto questo grado di sviluppo, continua ad ingrossarsi analogamente alle glandule acinose ed al fegato, vale a dire per gemme solide dell' epitelio dei calici, le quali regetando 
rigogliosamente e ramificandosi formano lo strato corlicale intorno ai calici raggruppato in lobi (renuncoli). (1)

Queste parti terminali si fanno poscia cave per fusione delle cellule interne, mentre nello strato fibroso esterno si sviluppano i vasi sanguigni.

I tubuli ramificati rettilinei formano la sostanza midollare; le loro estremità contorte coi rigonfamenti (corpuscoli del Malpighi) costituiscono la sostanza corticalc. Kölliker ha osservato in un embrione umano di circa quaranta giorni che i reni veri erano ormai costituiti da elementi tubulari e vescicolari.

\section{RENI SUCCEN'URIATI}

I reni succenturiati si sviluppano fra la sesta e settima settimana da un blastema che circonda l'aorta al davanti dei corpi del Wolf.

Nel secondo mese i reni succenturiati sono più voluminosi dei veri reni; nel terzo mese di gestazione invece cominciano ad arrestarsi relativamente nello sviluppo, mentre i reıi s'ingrossano assai rapidamente e così, come abbiamo veduto pel fegato, si stabiliscono gradatamente i rapporti di volume e di peso che si osservano nell' adulto fra questi due organi.

Per mie direlte osservazioni posso confermare quanto disse Meckel che nell'embrione di sei mesi il peso dei reni succenturiati in confronto di quello dei reni veri è di circa 2: 5; nel neonato $=1: 3$, e nell' adulto $=1: 8$ e più.

\section{SVILUPPO DELL' APPARECGHIO GENITALE INTERNO ED ESTERNO}

Le glandule genitali hanno origine autonoma da un blastema speciale che si vede apparire in ambedue i sessi al lalo interno ed un poco indictro dei corpi di Wolf.

(1) Nei feti umani ed anclie nel bambino appena nato i reni banno un aspetto heruoccoluto che scompare dopo la nascita. In alcuni auimali, come nel delfino, nell' orso, nel maiale, negli uccelli ec, conservano questo arpetto letale per tutta la vild extrauterina. 
Qurasi contemporaneamente si osserva a ridosso e nella linea mediana dei corpi del Wolf, e quindi all' esterno delle slandule genitali, un canale che termina superiormente in una vescichetta, il quale dal suo scopritore dicesi condotto del Miiller. Nei primi tempi il blastema che rappresenta i rudimenti delle glandule genitali è di forma rotonda, in seguito ingrossandosi si fa ovoidale, oblungo e comprimendo il rene primitivo lo fa, per cosi dire, atrofizzare; fin qui non vi ha alcuna differenza nè di rapporto anatomico, nè di struttura fra le glandule dei due sessi; ma in seguito i dottolini residui del corpo di Wolf si saldano colla glandula genitale, e se questa si trasforma in testicolo si mettono in comunicazione coi suoi canaletti seminiferi ed allungandosi, facendosi flessuosi ed aggomitolandosi, costituiscono l'epididimo; quindi il dotto escretore del corpo di Wolf diventa dotto deferente, che in basso presenta una dilatazione, la vescicola spermatica. I dottolini residui del corpo di Wolf, che non si misero in comunicazione coi canaletti del testicolo, formano i vasi aberranti dell Haller; il dotto di Müller, che abbiamo descritto, si atrofizza nel maschio dall' alto in basso e ne rimane soltanto l'estremità inferiore, che, fusa con quella dell' altro lato, viene ad essere circondata dal blastema della prostata e forma quella saccoccetta aperta all' innanzi che si osserva al fondo della stessa, utero mascolino.

Ben diversamente succede quando la glandula genitale diventa ovaia, poichè allora non si mette in alcun rapporto di continuità coi canaletti del corpo di Wolf, ma soltanto questi vi aderiscono all' esterno ed involgendosi anzichè sviluppandosi si riducono alle minime proporzioni d' un corpuscolo ovoidale, che rimane quasi indifferente presso l'ovaia nello spessore del mes-ovario e che si conosce in anatomia sotto il nome di organo del Rosenmüller. All'incontro i dotli del Müller si sviluppano allungandosi ed allargandosi all'estremita superiore, finalmente si aprono, come se scoppiassero, presentando un' apertura imbuliforme ed a bordo frangiato ( Morsus diabuli) libera nella ravilà peritoneale. 
Lo stesso succede all'estremila inf'eriore, dove questi dotli convergono tra di loro per formare una vescichetta comune, la quale s' ingrosaa per inspessimento delle sue pareli costituendo l' utero e forse parte della vagina.

La porzione superiore del dotto di Müller, che si conserva cilindrica ed è contenuta fra le pagine del leganento largo, costiluisce la luba faloppiana.

Fra il lerzo ed il quarto mese di gestazione le glandule genilali d' ambo i sessi incominciano ad abbassarsi verso la casità pelvica, prendendo una disposizione piutlosto inclinata convergente in basso; ma le ovaie si arreslano ben presto nel loro movimento, mentre i testicoli continuano a portarsi in basso seguendo nella loro discesa una guida formata da una ripiegalura dalla forma di una navicella, la cui prua, per continuare la similitudine, si prolunga in un cordone dirello che termina al canale inguinale (Gubernaculum Hunteri). Verso la fine della gravidanza i testicoli passaddo pel canale inguinale discendono nello scroto, c questo cambiamento di luogo dicesi descensus testiculorum: naturalmente il testicolo finchè trovasi nella cavilà addominalc è coperto dalla pagina viscerale del peritoneo, la quale alla periferia passa sugli organi adiacenti : e mano mallo che il testicolo si la pii sporgente, pel suo sviluppo, i margini della suddella pagina si avvicinano sempre più dietro di esso, lo involgono totalmente e laddove si saldano costiluiscono il mes-orchio. Nella borsa scrolale questo involucro forma la vaginale propria, intorno alla quale vi ha un prolungamento della fascia trasversa ed al. cune fibre del muscolo obliquo interno (cremastere) che il testicolo ha incontrati nell'altraversare le pareli addominali.

\section{GENITALI ESTERNI}

La porzione d'egestione del tubo gastro-enterico, che per corrosione del blastema si è messa in comunicazione coll' intestino intermedio, si dilata e forma la clnaca, nella quale sbocea posteriormente l' intestino rello ed anteriormente il 
seno uro-genitale, che riceve i dotti escretori dei corpi del Wolf e dei reni permanenti.

Ill alcuili animali vertebrati, come negli uccelli, si arresta lo sviluppo pressochè ill questo stadio, mentre nei mammiferi e nell' uomo si sviluppa un setto trasversalc da destra a sinistra, che divide il seno uro-genitale dalla cloaca. Questo setto, che all' esterno vienc ad essere coperto dai tegumenti comuni e dicesi perineo, si forma nel modo seguente : la cloaca presenta dapprima d'ambo i lati una ripiegatura, la quale sempre più s'innoltra verso la linea mediana e saldandosi finalmente con quella dell'al tro lato divide il seno uro-genitale dal retto. Prima però che si compia la divisione si osserva un sollevamento o sporgenza della porzione anteriore del Iabbro che circonda l'apertura della cloaca, il quale innalzandosi sempre più nella linea mediana costituisce il cosi detto promontorio, nel mezzo del quale vedesi stretla l'apertura che mena direltamente nel seno uro-genitale.

Ai lati di quest' apertura si vedon sorgere due ripiegature le quali si portano all' inmanzi e convergono fra loro formando nel punto dove s'iucontrano una sporgenza conica : vella donna rimangono sempre divise inferiormente costituendo le piccole labbra e la clitoride, che ne è la comınissura sporgente anteriore; mentre nell' uomo allungandosi e cliudendosi anche inferiormente dall' indictro all' avanti costituiscono il pene, che rinchiude il canale di escrezione del seno uro-genitale (uretra), mentre nella donna quest' apertura trovasi al di solto del clitoride e circondata dalle piccole labbra.

Ai lati di queste pliche, che diedero origine al pene od alle piccole labbra col clitoride, si formano due ripicgature de'tegumenti comuni, le quali sviluppandosi di molto vengono ben presto a coprire le precedenti, lasciandone sporgerc soltanto la commissura anteriore (cioè clitoride o pene); queste ripiegature o Iabbra convergendo tra loro si saldano superiormente ed inleriormente, limitando cosi uno spazio ellittico, alla cui 
estremiti superiore trovasi l'apertura dell' uretra, ed in sollo l'adito all' apparecchio genitale inlerno.

Nella douna queste due sporgenze costituiscono le grandi labbra che restano sempre divaricate; mentre nell' nomo ayvicinandosi al di sotto del pene Hella linea mediana si saldano tra loro e costituiscono lo scrolo, dapprima vuoto, e poi, fra il settimo o l'oltavo mese, accoglie i testicoli che sono discesi pel canalc inguinale.

Non si conoscono ancora le condizioni od i momenti causali che determinano i! blastema della glandula germinale a diventare testicolo piuttosto che ovaia, e ad indurre quelle modificazioni nelle parti genitali interne ed esterne da dare la differenza dei sessi. Alcuni pretenderebbero che vi abbia una graude influenza lo stalo di maturità dell' uovo quando riene fecondato, ritenendo che si sviluppa un maschio quando il !iquido fecondatore incontra un ovulo perfettamente maturo.Le mic esperienze, che per altro penso di continuare perchè non compiute, mi diedero fin qui dei risultati in parte farorevoli ed in parte contrarii a questa tcoria ed opinione, poichè dalle uova deposte da una partita di galline dopo otto o dieci giorni che era stato alIontanato il gallo si avevano a preferenza femmine che maschi, e molte, come abbiamo già veduto altro. ve, abortivano; invece le uova deposte dalle galline nel secoudo e terzo giorno che erano col gullo davano quasi l'egual numero di maschi e femmine. E credo di aver osservato piuttosto delle differenze circa il numero dei maschi e delle fommine dipendenti dalla stagioue e dall' alimentazione anziclıc̀ dalle diverse condizioni o rombinazioni d'accoppiancuto.

SVIIUPPO DEGII ORGANI DEI SENSI

ORGANO DELLA VISTA

Gli occhi si originano da due piccole vescichette (o pure da una che poi si divide in due) che si vedono sorgere latcralmente ed inferiormente nel solco che divide il prosencefalo 
dal mesencenfalo. Le pareti di quente rescichette si continuano col blastema che forna le resciche cerebrali colle quali stanno irr aperta comunicazione. In seguito le vescichelle s'ingrossano, si famo sporgenti allo innanzi, rimanendo peró sempre unite mediante un peduncolo cavo col cervello primitivo.

Il blastema di sostanza nervosa clie costituisce queste rescichette s'irvolge, come le vescicole cerebrali, dello strato fibroso e corneo epidermoidale comune. Quest'ultimo in corrispondenza del putto piil culminante delle vescicole s' illspessisce per proliferazione di cellule, ed introflettendosi forina come un clavo sferico vuoto, il quale oppone una certa resistenza allo sviluppo della vescicola, e fnalmente s' infossa nella stessa introflettendone le pareti, le quali si conformano a sacco doppio, che lo circondano ed infine lo rinchiudono. Cosi si forma una capsula di sostariza nervosa a doppia parete, retina, che racchiude un ammasso di cellule cornee, dal quale si forma anteriormente la lente cristallina, e posteriormente ed ai lati, per fusione di cellule, l'umor vitreo.

Le pareli doppie di sostanza nervosa per l'introduzione dell' ammasso di cellule corrree vengono a mutuo contatto, si fondono tra loro e cosi scompare la cavità primiliva della vescicola, la quale era distesa prima da un liquido; il peduncolo diventa solido e rappresenta il nervo oltico. Insieme all'introflessione anteriore della vescica si forma un solco al lato interno inferiore che dal punto introflesso della vescicola si continua all' indietro sul pedunculo o nervo ottico.

Lo strato fibroso che trovasi immediatamente in contalto della vesciclictta primitiva, si fa presto vascolare, e per deposito di pigmento granuloso nelle cellule di connettivo vienc a costituire la coroidea, che, come è naturale procede dal peduncolo verso il polo anteriore della vescichetta. In corrispondenza del solco al margine interno inferiore si osserya per molto tempo un' interruzione dello stroma della coroidea fessura coroideale la quale in seguito si restringe e si chiu- 
de. Lo strato piì esterno della pagina fibrosa si corsolida e costituisce la sclerotica e la cornea. Intanto il globo oculare viene rinchiuso nella cavità dell'orbita, la quale si forma per l'incoutrarsi del processo frontale laterale col processo superiore del primo arco branchiale. I tegumenti comuni che rivestono all'esterno i bordi della cavita orbitale formano due pliche semilunari (palpebre), le quali crescendo ed avvicinandosi reciprocamente coprono sul davanti l'occhio.

Durante la vila embrionale sono assai sviluppati i vasi sanguigni interni del bulbo oculare; un'arleria ed una vena clie partono dalla coroidea in corrispondenza del nervo ollico, attraversano l'umor vilreo dandovi dei rami e finalmente raggiungono la lente cristallina, intorno alla quale si forma una rete vascolare per l' anastomizzarsi dei rami dell' arteria e della vena centrale coll altri provenienti dalle parti anteriori. Nell'uomo i vasi della capsula vascolare che circondano la lente cristallina si obliterano verso l'ollavo mese della vila intrauterina, mentre in alcuni animali, specialmente nei carnivori che nascono cieci, la scomparsa della capsula vascolare della lente succede alcuni giorni dopo la nascita.

ORGANO DELL'OLFATTO

Alla faccia inferiore dei lobi anteriori del cervello primitivo veggonsi sorgere due processi cavi. Questi processi terminano anteriormente a modo di clava e si dirigono verso due fosselle lapezzate dal foglietlo corneo-epidermoidale, che si formarono nella capsula fibrosa cranica ai lati della linea mediana anteriore della stessa, un poco più innanzi ed in basso degli occhi ed immediatamente sopra dell' ampia apertura orale. Queste due fossette circondate in principio da un Iabbro circolare poco sporgente si fanno sempre più profonde ed a poco a poco diventano oblunghe verticalmente, finclì̀ per corrosione od introflessione del loro margine inferiore, clie scompare (solco nasale), si mellono in comunicazione colla cavila 
viale, dalla quale inseguito vengono ad essere divice, come ab!riamo giil vedulo, per lo sviluppo dell'osso mascellare e delle ossa palatine che formano la volta della bocea ed il pavimento delle cavilà nasali. La separazione delle fossetle nasali dalla bocea, od il processo di chiusura inferiore delle prime, incomiucia dall'esterno. Lc duc clave di sostanza nervosa infine peuetrando uci fondi ciechi delle fosselte primilive, clie col farsi profonde si sono trasformate in canali larghi superiormeute (regionc olfalliva) e strelli in basso (regione respiratoria), vengono a saldarsi col foglicllo mucoso che dalla cavitì orale è passato a lapezzare le fosse nasali. Questa porzione che viene chiusa nclle fosse nasali costiluisce l'estremità periferica del ucrvo olfattorio nella membrama olfatliva; il rjmancnte della clava che resta nel cranio, e comunica col cervello mediante il peluncolo, forma il nervo ulfatliso.

Le piune nasali incominciano a formarsi alla fine del secondo mese per lit sporgenza dei processi nasali dell'osso fronlale che sono coperti daj legumenti comuni. E pertauto il naso $\mathrm{e}$ in principio assai piallo e le aperture delle narici sono nel piano della faccia ed assai distanti fra loro; ma sollevandosi in seguilo il processo mediano ed avanzaudosi i processi laterali, il naso acquisla man mano la sua forma tipica c le narici cangiano di direzione volgendosi in basso.

D'ambo i lati dell' epencefalo all' altezza del sccondo arco branchiale si scorge da principio una fossella (formalasi per introflessione del foglietto cornco-cpidermoidale) la quale ficendosi sempre piu profonda e chiudendosi anteriormente, per l'avvicinarsi dei suoi bordi, si trasforma in una vescicola sferica che diccsi vescicola del labirinto, appunto perchè si trasforma in progresso di sviluppo nella sezione iuterna dell' organo dell' udito che porta questo nome. La descrilla rescicola si solleva ed isolandosi dall' avanti all' indictro dai tessuli cir- 
costanti diventa per tal modo piriforme; ed in fine si congiunge mediante il peduncolo direlto all' interno con un processo proveniente dall' epencefalo e quindi si mette in rapporto direlto coll'asse cerebro-spinale.

Una volta che ha acquistato la forma ed i rapporti descritti, anzi durante questi processi, la vescicola si è uaturalmente dilatata ed inspessita nelle suc pareti ; allora si originano all'esterno dei solchi in diverse direzioni che vanno sempre approfondandosi c ne mutano essenzialmente l'aspello esterno e la forma della cavità, la qualc resta divisa in tre concamerazioni comunicanti tra loro c corrispondenti al vestibolo, all'acquedollo del veslibolo ed alla coclea. Nel primo si formano"i tre canali semicircolari da tre ripiegature disposte in vario senso; il secondo si allunga assumendo la forma di un scmplice canalc (acquedollo) ; nella terza le pareti presentano una ripiegatura circolare disposta a spirale (coclea). Tutle e tre queste rescicole vengono a rivestirsi della pagina fibrosa che si ossifica.

La cavità del timpano si forma da quello spazio che rimane posteriormente tra il primo ed il secondo arco branchiale innanzi alla vescicola del labirinto; questo spazio si mette in comunicazione anteriormente colla retro-bocca mediante un cauale (tromba d'Eustachio) pel quale si continua il foglio mucoso che va a tapezzare internamente la cavilà del timpano. Gli ossicini dell'udito ed i loro muscoli si formano, come abbiano già veduto, dalle porzioni del primo e secondo arco branchiale che restano chiuse nella cavila del timpano sviluppatasi intorno ad cssc. Stante il precoce sviluppo e lc grandi dimessioni degli archi visccrali in confronto delle parti adiacenti, gli osaicini dell' udito che si formano da quelli hanno già nel feto quasi le dimensioni che si osservano ucll' adulto. II processo d'ossificazione comincia ben presto in questi ossivini ' si rompic quasi interamente durante la vita intranteriua.

Il blastema che rappresenta i legumculi commui mellit ir sione temporale presenla ben presto mo infossamento cirron 
dato da un labbro sporgente, il quale sviluppandosi forma il padiglione dell'orecclio; questo dapprima è piano e poscia presenta i rialzi e le sinuosità clıe ricevono in anatomia dei nomi particolari. L'infossamento centrale del padiglione si fu sempre più profondo fino ad incontrare la cavità del limpano, dalla quale resta diviso mercè la membrana timpanica.

Quanto abbiamo detto sullo sviluppo della boeca e della lingua ci dispeusa qui di parlare dell'organo del gusto.

Per l'organo del tatto ei limiteremo a dire che viene ad essere formato dal foglietto esterno o corneo-epidermoidale e dallo strato superficiale del foglielto medio o motorio-germinativo, che saldati insieme costituiseono i tegumenti comuni e le loro introflessioni che eoprono i margini delle aperture naturali o tapezzano per un eerto tratto le pareti delle prime vie della digestione, della respirazione, dell' apparecehio genito-urinario ece.

Per moltiplicazione delle eellule del foglictto esterno si formano i lîversi strati dell' epidermide e del reticolo Malpighiano sottoposto. I peli, le unghie, le glandule sudorifere, scbacee ece. sono produzioni di ‘uesto strato di cellule che ammassandosi e stipandosi s'innoltrano sempre più verso lo strato superficiale del foglictto mediano, lo introflettono e se ne involgono in modo da costituirne per eiaseuna una specie di guaina.

Clıe veramente queste glandule ed appendici eutanee sicno originate (lal foglictto esterno lo si prova macerando i tegumenti comuni d' un feto per stacearne dei lembi d'epidermide, la quale presenta la faccia in feriore tutta villosa per le appendici cornee, le quali per la loro forma si riconoseono come peli, glandule sebacee, sudorifere.

Anche la glandula inammaria è una produzione dello stesso foglietto ed in principio si presenta come una fossetta a pareti inspessite costituite unicamente di cellule dello strato corneo; in seguito si fa più grande e profonda ed origina de'processi che a modo di raggi si dipartono dal salo fondo ed in seguito si ramificano per gemme inuoltrandosi sempre nel lessuto eonneltivo dello strato soltoposto che l'involge e li circonda coi suoi vasi. Per fusione delle cellule'interne i dolli si famo cavi a per spor- 
genza di bordi della lussuta primitiva si forma il capezzolo. $\Lambda$ ll' eproca della nascita i doltolini delle glandule inammarie d'ambo i sessi con-tengono un umore lattiginoso che in alcuni paesi, forse in luase ad un pregiudizio popolare, si usa spremere dai capozzoli de"zneonati e si riconosce col nome di latte della strega (Hexenmilch).

\section{APPARECCIIO VASCOLARE E CIRCOLAZIONE DEL FETO \\ NEI VARII RERIODE DELLA VITA INTIRAUTERINA.}

Tra la parete anteriore della porzione superiore dell'intestino e le piastrine laterali, che rappresentano le pareti del corpo, vedesi comparire il primo rudimento del cuore, mentre i primi vasi si formano tra le due pagine del foglietto mediano dell'area germinativa. Tanto questi quanto il cuore sono rappresentati iu principio da ammassi di cellule embrio-plastiche; i primi allungandosi e sviluppando dei processi formano dei cordoni solidi ramificati, i quali coi loro rami vengono ad incontrars $i$ ed a costituire una rete. Le cellule embrioplastiche che stanno alla periferia di questi cordoni si saldano tra di loro e formano per tal modo uno strato periferico (parete membranacea); mentre le cellule che trovansi nell' asse rimangono disgregate tra loro (globuli) e si vedono nuotanti in un liquido che si raccoglie allo interno della parete o per fusione di alcune cellule centrali o penetratovi per diffusione (1).

Il primo rudimento del cuore si presenta sotto la forma di

(1) Secoudo alcuni autori il sistema vascolare primitivo si formerebbe da un foglietto particolare, interposto fra le due pagine del foglietto mediano, che essi rhiamano vascolare od angio-plastico. La formazione dei primi vasi sanguigni nell'urea vascolare avverreble nel modo seguente. Un liquido incoloro interponeudosi come un essudato fra i foglietti li scolla ed accumulandosi in alcuni punti forma delle lacune; queste metlonsi ben presto in comunicazione tra loro, cosi che il liquido in esse contenuto possa passare dall' una all'altra. NIcune cellule si organizano negli intervalli ove non si è accumulato il liquido, altre si saldano tril di Joro pel lormare le pareti delle lacune; in questo inodo si costituiscono non solo le pareti dei vasi sanguigni in a benanche delle membrane inferposte ai foglexti, le ๆ119li servono a sostenere i rasi. 
un cauale cilindrico dalla cui estremita superiore si staceano due tronchi (aorte primitive) che piegandosi ad arco si dirigono dapprima all'indietro ed ai lati dell'intestino, indi decorrono in basso at di sotto della corda dorsale ed ai lati delli stessa tentendo a convergere fra loro verso la linca mediana per formare il tronco dell' aorta.

Da questi due tronchi si dipartono più o meno ad angolo rello tanti rami laterali, i quali portandosi all' esterno ed oltrepassando l'area embrionale si mellono in comunicazionc colla rete vascolare formatasi tra le due pagine del foglietto mediano dell'area germinativa opaca. Alla periferia di questa rete, che non raggiunge il limite esterno dell' area opaca, si osserva un grosso vaso terminale, il quale gira tutl'all' iutorno del rudimento del corpo embrionale, ed all'estremilì cefalica presenta un' interruzione, perchè s'introflette d'ambo i Iati onde portarsi fino al livello dell'estremo inferiore del cuore. Questo grosso vaso circolare, detto seno terminale, raccoglie tutti i vasellini della rete vascolare, la quale è lormata da due strati, uno superiore più esile, delicato, a rami più sottili (corrispondenti alle ramificazioni arteriose) ed a maglie più strette, ed uno inferiore ad ampie maglie, limitate da vasi più grossolani. I vasi di questa rete inferiore si raccolgono e mettono foce iuferiormente in due grossi vasi esterui e laterali al corpo dell' embrione e superiormente terminano nella porzione introflessa del seno terminale, la quale a livello dell' estremo iuferiore del cuore si unisce col vaso inferiore del lato corrispondente, ed origina cosi un tronco trastersale il quale, convergendo con quello del lato opposto, sbocca nel cuore: la rete inferiore rappresenta cosi il primo sislema venoso compreso fra il cuore ed il seno terminale : meutre la rete superiore, compresa tra il seno terminale e le aorte primitive, rappresenta il sistema arlerioso.

Appena formate le pareli del cuore (che assai presto direntano doppie e spugnose) sebbene non prisentino ancord tracce di elementi contrattili muscolari e molto meno di elementi 
nervosi, pure incominciano a contrarsi manifestandn dapprima un leilto morimento ondulatorio irregolare, il quitle a poco a poco si fa rilmico, regolare e piu frequente; e cosi s' inizia la prima circolazione, in quantochè il cuore, che si ì illungato e leggermente curvato, spinge nelle aorte e per queste nella rele vascolare superiore il liquido in cui sono sospese Ic cellule embrioplastiche disgrugate, che a poco a poco assumono i noli caralleri ici corpuscoli rossi del sangue (1).

Giunto il liquido nel seno lerminale prende la via della rete inferiore, che lo riporta al cuore pei due tronchi venosi inferiori.

Quesla prima e semplice circolazione dicesi circolazione dellarea vascolare la quaie, siccome si trova a ridosso della porzione del blastoderma che si costitnisce in vescicola vitulina od ombelicale sulla quale si distende, cambia ben presto li nome e dicesi circolazione della vescicola ombclicale od omfals-meseraica. Questa circolazione dura per lulta la vita embrionale negli ovipari, mentre nei mammiferi si arresta ben presto, c nell'uomo i suoi rasi si obliterano fra la setlima el ollava sellimana. Il processo di obliterazione dei rasi sanguigni incomincia già nel momento in cui si sriluppa la rescicola ombelicale, sulla quale viene a distendersi l'aren vascolare, clic ricere il sangue da duc soli tronchi arleriosi provenienti dalle aorte, e quando queste si sono riunite in un sol tronco per Cormare l' aorla addominalc, alla vescirola ombelicale si porta una sola arteria omfalo-meseraica, dalla quale si stacea un ramoscello che si distribuisce ai visceri addominali. Riducendusi in seguito la circolazione omfalo-rneseraica per lo sviluppo di quella dell'allantoide, c svi-

(1) Le cellule embrioplastiehe nuolanti uel plasma contenuto nei prini vasi sangugni si anmentano grandemente in mumero ed acquistano i caratteri di veri gobuli rossi del sangue coll'apparire del fegato primilivo, che sembra un focolaio degrli stessi, onde è molto probabile che nei primordii della vita embrionale le fimkioni di quest' organo sieno diserse che nell' aluln. 
luppandosi invece il tubo gastro-enterico e le glandule annesse, il ramoscello acquista beı preșto tali dimensioni da rappresentare il tronco principale, da cui si stacca, come ramo secondario, l'arteria omfalo-meseraica, la quale verso la settima setlimana ì ridotta ad un cordone.

Quanto abbiamo delto per le arterie vale anclie per le vene, le quali si riducono in numero, finchì da ultimo si ha una sola vena, che, provenendo dalla vescicola ombelicale, dicesi veria omfalo-meseraica. Questa appena penetra nella cavita del corpo dell'embrione riceve la vena del tubo gastro-enteri. co (Porta primitiva) colla quale resta circondata dai lobi del fegalo, cui di molti rami adveenti, ne riceve i reveenti e poi sbocca nel cuore.

Il passaggio da questa prima e semplice circolazione quasi extrafetale, alla forma e grado di sviluppo dell' apparecclio circolatorio che presenta il feto all' epoca della nascita, è il risultato di molte circostanze di cui accenneremo le più generali e ben note, cioè: $\dot{1}^{\circ}$ lo sviluppo generale dell'embrione in tutte e tre le dimensioni e la chiusura dei tre lubi (speco vertebrale e cranio, tubo gastro-enterico, e cavità del corpo): $2^{\circ}$ i inutamenti di forma del cuore e la sua divisione in diverse cavità distinte: $3^{\circ}$ la comparsa di alcune parti od organi nuovi (p.e. l'allantoide, le estremita ecc.) e l'arresto di sviluppo o la scomparsa di altri (p.c. la vescicola ombelicale). E perciò i tronchi principali primitivi arteriosi e venosi (appartenenti agli organi che s' arrestano nel loro sviluppo o scompaiono) diventano rami delle loro proprie diramazioni, le quali, mutandosi i rapporti di sviluppo, subentrano non solo al posto dei tronchi principali primitivi (ridotti od obliterati) ma benanco mulano i rapporti anatomici delle diverse parti del sistema vascolare.

Partendo da queste premesse esamineremo dapprima lo sviluppo del cuore, che abbiamo lasciato allo stato di un tubo cilindrico, leggermente incurvalo a ferro di cavallo, già pulsante e sporgente (punctum saliens) al di fuori della cavita ed 
corpo sotto del cappuccio cefilico ed al davanti del foudo cieco superiore della porzione digerente del tubo gastro-enterico.

Probabilmente per l' allungamento del cuore in uno spazio limitato e per le resistenze che incontra in alto ed in basso intumeuta man mano la sua curvalura, e per ciò l'estremilà superiore, che d'ora inmanzi diremo arteriosa, si porta in basso ed all' innanzi, mentre l'inferiore o venosa si piega in alto portandosi al di dielro della superiore; c cosi le diverse parti del cuore inflesse l'una sull' altra vengono a trovarsi iu diversi piani nel senso antero-posteriore.

Mentre il cuore in tale modo si piega ad ansa, in corrispondenza degli angoli presenta degli strozzamenti, o per meglio dire si formano delle dilatazioni nei tratti compresi fra i punti d'inflessione.

Cosi il tronco arterioso incomincia con un rigonfiamento, bulbo arlerioso, ed al di sotto di questo vediamo uno stringimento, cioè un solco orizzontale piì marcato a destra che a manca, il quale lo divide dalla parte più bassa anteriore che è assai rigonfiata per prolrusione della parele anteriore (ventricolo); posteriormente al ventricolo ed a sinistra si osserva uı terzo rigonfiamenlo che sta al dorso dei due primi (alrii); infine vediamo due rigonfiamenti vescicolari (orecchielle) all'estremilà venosa, precisamente in corrispondenza del punto ove shocca il tronco venoso proveniente dall'area vascolare o dalla vescicola blastodermica. Continuando nello stesso senso i cangiamenti dei rapporti anatomici dei rigonfiamenti primitivi del cuore, che seguilano a svilupparsi, la parte venosa che cra la piì bassa viene col tempo a trovarsi in alto, abbracciando dal di dietro colle orecchielte il bulbo arterioso, mentre il rigonfiamento che corrisponde al ventricolo si porti sempre in basso ed acquista spazio a sinistra (1).

(1) Tulto questo venne osservato e studiato sugli embrioni degli necelli c dei manmiferi. Soltanto Coste deserive il cuore d'un embrione unano dí circa 15 a 18 giorni in cui lo trovê pieğato ad $s$.

Eeker ne deserive nno d'un embrione di tre o quattro seltimane in cui erano formate tutte le parti sopradescritte. 
E qui è a notare che una volta formato l'angolo di divisione fra il ventricolo ed il rigonfiamento auricolare, l'ondil sallguigna venosa giungendo nel cuore deve urtare contro la purete anteriore del ventricolo e distenderla; perciò la parete posteriore si arresta nello sviluppo, e, come facilmente si comprende, deve scomparire in ragione che gli atrii s'innalzano e si portano in avanti per abbracciare il bulbo artrioso. E perciò può dirsi che il ventricolo realmente non è formato dà altro che dalla sua parete anteriore, distesa all' innanzi a mo' di saccoccia dall' onda sanguigna.

La formazione dei setti per dividere queste tre cavità, comunicanti tra loro, in una metà destra o venosa ed in uıa metà sinistra od arteriosa, incomincia verso la quarta settimana e precisamente dapprimi nel tronco arterioso, ove si vede sorgere dalla parete posteriore una duplicatura longitudinale leggermente flessuosa, la quale sempre più s'innalza, e finalmente iucontrando la parete anteriore divide il lume del tronco in due, di cui il destro rappresenta l'arteria polmonare, il siuistro l'aorta: da questo momento il sangue che esce dal ventricolo è obbligato a prendere due direzioni (1). Più tardi avviene lo stesso nel ventricolo, dalla cui parete posteriore si innalza un sepimento falcato, colla concavità allo innanzi ed in alto, il quale si avanza a poco a poco e completa la divisione del rentricolo primitivo in due metà; peró finchè le pareti dei ventricoli non si sono inspessite e le fibre non hanno acquistato il vero carattere di fibre muscolari, rimane in alto un piccolo foro di comunicazione, che in seguito vien chiuso per una specie di centro tendineo nell'uomo e per una cartilagine che poi ossifica nei ruminanti.. Alla fine della quinta settimana nell'embrione umano è completata la divisione del tronco arterioso e dei veutricoli. La divisione del rigonfiamento posteriore venoso si verifica ancora più tardi per lo sviluppo

1) Negli anfibii questa ripiegatura sigmoide del tronco arterioso non si sviluppa al punto d'incontrare la prarete anteriore e percio la divisione riin ane incompleta. 
d' un setto clie incornincia ad innalzarsi dalla porzione superiore del setto ventricolare già formato, s'innoltra dal basso in alto e dall'avanti all'indietro niella cavità degli atrii, ove si incontra con una duplicatura interna della parete degli stessi, la quale incomincia in corrispondenza del solco che divide le due orecclisette.

La totale chiusura del setto degli atrii non si verifica che dopo la nascita; e non sono rari i casi in cui rimangono per tutla la vita, come difelto di sviluppo, una o più lacune nel setto, per cui le due cavità degli atrii rimangono sempre comunicanti tra di loro.

Nel felo la maggiore pressione è esercitata dal sangue venoso portato dalla vena omfalo-meseraica o dalla vena ombelicale, mentre quello che ritorna dai polmoni ancora inattivi è in piccola quantità, e perciò esercita poca pressione. Una rolta però che è iniziata la respirazione si aumenta istantaneamente la quantità e perciò la pressione del sangue che si versa nell' atrio di sinistra, e quindi viene sbattuta e compressa contro gli orli della lacuna ancora esistente (forame ovale) una ripiegatura valvolare della parete dell'atrio sinis/ro, la quale aderendo e saldandosi agli orli chiude la comunicazione tra i due atrij.

Le pareti del ventricolo in principio sono molto sottili e formate da cellule nucleate stellate, le quali unendosi coi loro processi costituiscono un tessuto spugnoso molto lasso, che in seguito poi si fa compatto e finalmente diventa liscio e levigato alla faccia esterna.

Il setto dei ventricoli in principio trovasi molto a destra e perciò il ventricolo sinistro è molto più ampio del destro, ma a poco a poco si stabilisce la proporzione di capacità di entrambi.

Gli apparecchi valvolari sono rappresentati in principio da trabecole membranacee, gelatinose, molli, che in seguito diventano compatte e resistenti per sviluppo di tessuto elastico. Le valiole semilunari dell'arteria aorta e polmonare in prin- 
cipio sono formate da tre cordicine trasparenti tese nel lume del vaso, che si toccano colle estremili ove s'inserisconu alle pareti ; per la presenza di queste cordicine il lume del vaso diventa triangolare; in prosicguo si distendono e diventando laminari formano le tre saccocce (1).

I noduli alle valvole semilunari dell'aorla e dell'arteria polmonare, non clie quelli da me scoperti alcuni anni fa ai margini delle valvole atrio-ventricolari sono assai appariscenti nei neonati, perchè tumidi.

\section{SVILUIPO DELLT ARTEIIL:}

Passando ora noi allo sviluppo de'vasi incominceremo dalle arterie, limitandoci all'esame dei tronchi arteriosi principali.

La forma prima è quella già descritta, cioè d' un tronco arte. rioso il quale si divide in due rami che piegandosi ad arco si portano verso la colonna vertebrale ; ivi giunti si piegano in basso c decorrono ai Jati della stessa verso l'estremità caudale dell' embrione. Per qualche tempo rimangono divisi, ma in seguito si fondono in un sol vaso arterioso (aorla toracica ed addominale) (2).

Ritorniamo ora ai nostri primi archi dell'aorta, che abbiamo fin da principio, cioè quando il cuore stesso colla parte superiore arteriosa sta immediatamente sotto del cervello alla base lel cranio. Noi sappiamo però duc cose cioè: $1 .^{\circ}$ che il cuore a

(1) Il cuore quale centro della circolazione, che è la funzione prineipale delli vita vegetaliva, preeede nello svilup po tutti gli altri organi del corpo e perfino il fegato. Verso la terza e quarta settimana ocenpa intla la ravití toraciea dell'embrione, ed il suo peso sta a quello del corpo come 1:12. Ingrossandosi in seguito gli altri organi, sebbene il cuore continui anclıe a svilupparsi, pure relativamente dimiuuisee in peso ed in volume rispetto a quello del eorpo; e perciò nel ter\%o mese forma la einquantesima parte drl peso di tutto il corpo, e nel neonato il suo peso sta a ruello del corpo co-

(2) Talvolta pérò lal divisione si mantiene per tulta la vila. Allen THom-

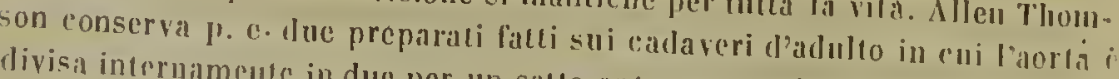
divisa interuameute in due per un selen antero-pnsteriore completo. 
poco a poco si porta in basso staceandosi dalla base del cranto, perche tra questo ed il tronco si forma la regione cervicale; $2^{\circ}$ che in questa regione si formano gli archi viscerali o lranchiali per corrosione del blastema che forma le pareti dell' inbuto d'ingestione.

E pertanto dopo questo cangiamento di sito del cuore e dopo lo sviluppo degli archi branchiali noi abbiamo un allungamento del tronco arterioso o meglio dei primi due arehi, che rimasero colla loro sommita alla base del cranio, mentre la radice del tronco arterioso si è portata in basso e le branche si sono allungate.

Allora si vedono sorgere dei tronchi anastomolici fra le due branche, per cui si ha la formazione d'altreltanti archi che chiamansi secondo l'ordine in cui comparvero primo, secondo, ter $\approx 0$, quarlo, ecc. $\mathbf{b}^{\prime}$ ordinario se ne contano da tre in quatlro, poichè quando si sviluppa un' anastomosi inferiore si oblitera a poco a poco un arco superiore, e cio è ben naturale per ragioni meccaniche-idrauliche facili a comprendersi $(1)$.

E bene però che io ricordi come per l'allungamento della regione cervicale gli archi branchiali debbano anche allontaIIarsi fra loro.

Nei pesci questa forma del sistema arterioso si mantiene per tutla la vita, colla differenza però che l'aorta non viene ad essere costituita dai tronchi arteriosi, ma questi ramificandosi nelle branchie si risolvono in capillari, dai quali sorgono delle vene che convergono e sboccano nella vera arteria aorta co!locata assai profondamente, cioè sulla colonna vertebrale.

Ma ne' rettili, negii uccelli o nei mammiferi il tipo embrionale degli arcli aortici subisce varie modificazioni e, conte $\dot{c}$ naturale, i mutamenti più forti li passa nell'uomo c nei mammiferi superiori.

(1) Gli archi aortici diconsi anche archi branchiali, poiche si trovano m enrispoudena delle lamine biserali o branchiali. 
Ecco press'a poco cosa succede secondo quello che descris. sero Baer e Ratke.

Dalla sommita del primo arco si staccano due rami che si portano nel cranio. Il sangue prende quindi la via di questi rami, ed il trutto del primo arco compreso fra l'uno e l' altro si oblitera; il ramo anteriore rappresenta la carotide esterna ed il posteriore la carotide interna: si oblitera pure il secondo arco branchiale per l'allungarsi della branca interna, la quale rappresenta l'origine della carotide esterna mentre il terzo arco è l'origine della carotide interna.

La carolide comune è rappresentata dalla branca mediana tra il terzo e quarto arco.

A destra la carotide comune e la succlavia sorgono da un tronco che è il residuo della branca tra il quarto e quinto arco branchiale obliterato; in tal modo l'origine della succlavia è rappresentala dal quarto arco, mentre a sinistra la succlavia e la carotide comune si distaccano divise all'estremità del quarto arco, che rimane e rappresenta l'arco permanente dell'aorta.

Il quinto arco, che scompare a destra e rimane a sinistra, forma l'arteria polmonare, mentre la sua branca esterna o posteriore, clie si continuava in alto col quarto arco, forma il dollo del Bolallio; dalla concavità del quinto arco di sinistra si vedono staccare le piccole arterie polmonari.

Una volta esposta l'origine dei trouchi principali arteriosi, pare inutile seguire dettagliatamente lo sviluppo dei loro rani, che ha luogo a misura che le parti si formano.

Per la fusione delle due aorte primitive in una sola aorta si spiega come le arlerie omfalo-mesenteriche diventino rami dell'aorla addominale impari, i quali l'un dopo l'altro si obliterano finchè ne rimane soltanto il destro, da cui si stacca in principio un piccolo ramo clie va all' intestino (arteria mesenterica superiore). Questo, pel l'obliterazione successiva dell' arleria omfalo-meseraica, diventa ramo principale dell'aorta. 
Le aorte primitive terminano in basso. ramificandosi nelle pareti della vescicola allantoide; ma una volta clie le due arterie aorte si sono fuse ill un sol tronco, le arterie dell'allantoide rappresenlano i rami terminali principali dell'aorta, poichè tra l'una e l'altra si vede soltanto la piccola sacrale media, che ne sarebbe la vera continuazione.

Questi due tronclıi principali terminali dell'aorta cliamansi arlerie ombelicali, le quali appunto servono a portare il sangue nelle pareti dell'allantoide e quindi nel corion villoso e nella placenta. Quando incominciano a comparire gli arli posteriori, in vicinanza del punto di biforcazione dell' aorta nelle due ombelicali, sorge d'ambo i lati ed all' esterno un ramoscello arterioso (arteria iliaca primitiva) che a poco a poco si allunga e s'ingrossa insicme all'arto, siccliè all'epoca della nascita queste due arterie hanno raggiunto tale sviluppo in confronto delle ombelicali, da rappresentare esse la continuazione dell'aorta, mentre le ombelicali sono ridotte a semplici diramazioni delle iliache comuni.

SVILUPPO DELLE VENE

Il sistema venoso primitivo è piuttosto extrafetale, cioè appartenente all'area vascolare; lc prime vene omfalo-meseraiche riunite in un breve tronco sboccano nell' estremità venosa del cuore. Coll' addossarsi e distendersi dell' area vascolare sulla vescicola vitulina e col chiudersi del canale intestinale scompaiono a poco a poco le vene, e finalmente sul peduncolo della vescicola si vede decorrere insieme all'arteria una sola vena omfalo-mesenterica, la quale appeIra entrata nella cavità del corpo dell' embrione riceve una vena proveniente dall' intestino (vena mesenterica) e poi viene compresa dai lobi del fegato; peró mentre questo succede, nel corpo dell' embrione si svilupparono altre vene, delle quali alcune appartengono agli organi interni ed altre alla circolazione dell' allantoide; quelle di quesl'ulli- 
tha riunite in un solo ramoscello sboccano nell' unica rena omfalo-meseuterica, e siccome le vene dell' allautoide, specialmente nei mammiferi, hanno uno sviluppo rapido, cosi succede clie la vena dell'allantoide od ombelicale rappresenta ben presto il tronco principale che va al fegato, cui porta il sangue reduce dall'allantoide, dall’intestino e dalla rescicola o mbelicale. Nel fegato si sviluppano da questo tronco venoso le vene adveenli e le reventi. Tutto il sangue però della vena ombelicale non passa nelle vene adreenti, ma per la maggior parte va direttamente al cuore per la continuazione della vena ombelicale che scorre solto del fegato al cui bordo posteriore riceve gli shocchi delle vene reveenti. Questo tratlo della vena ombelicale sotloposto al fegato diccsi dotto dell Aranzio.

Ai lati della testa e del collo dell'embrione si sviluppano le vene giugulari contemporaneamente e forse prima ancora clie si sviluppino i vasi dellallantoide; lo stesso succede per la parte inferiore del corpo, in quantochè ai lati cd al di sotto della colonna vertebrale si vedono formare le due vene cardinali, le quali giunte al livello del cuore si uniscono colle giugulari del lato corrispondente formando un tronco trasyersale (dollo del Cuvier) che sbocca nel cuore con quello del lato opposto c colla terminazione della vena omfalo-mescraica che è diventata ombelicale. Dopo qualche temipo si sviluppa la cava inferiore a destra dell' aorta da due radici che si anastomizzano colle vene cardinali; questa cava inferiore sbocea nel tronco della vena ombelicale dopo che questa lia ricerute le vene epatiche revecnti. Perciò dopo formata la vena cara inferiore, tutte le vene dell'embrione ed extracmbrionali sboccano in una specie di seno venoso che inctte nel cuore, e siccome a poco a poco questo scrio viene a far parte dell'atrio, i dotti del Cuvier vengono a disporsi verticalmente c chiamansi cave superiori, mentre il piccolo tronco formato dalla unione della cava inferiore colla vena ombelicale slsocca isolato nell'alrio destro rappresentando l'estremiti della cara ascen- 
dente. Pii tardi il sistema della cava smperiore sinistra si unisce con cytello della destra e sboeca con un solo tronco net euore; la vena cardinale sinisla diventa la vena emi-azygos e la cardinale destra prende il nome di azygos; come pure l'estremita cardiaca del dollo di Cuvier di sinistra forma lí vena coronaria del cuore.

Come si rileva da quanto si disse a pag. 74, 118 e 127, l'aorta addominale primiliva termina biforcandosi in due arterie, le quali si ramificano sulle pareti della vescicola allan. toide ore formano una rete abbastanza filla; il sangue reduce da questa si raccoglie in una rena che sbocca nell' omfalomeseraica. Col cessare della circolazione della vescicola vitulina od ombelicale (ciò che nell' embrione umano si verifica verso la seltima seltimana della vila intrauterina) il tralto della vena omfalo-meseraica, che giace fra lo sbocco della vena dell'allantoide ed il cuore, continua a svilupparsi, rimaneudo pervio per dar corso al sangue reduce dall'allantoide.

Le arterie che portano il sangue dall' aorta alla rete della vescicola allautoide e le rispeltive vene che lo riconducono al cuore prendono il nome di vasi ombelicali. Chiudendosi le pareti addominali l'allantoide viene ad essere divisa, per uno strozzamento mediano, in due parti, l'uma interna od addominale che diventa vescica urinaria, l'illtra esterna che è l'allantoide vera. L'ulteriore saldamento delle pareti addominali procedente dal basso in alto verso l'anello ombelicale, che si chiude e si allontana sempre più dal pube (1), riduce la parte dell'allantoide compresa nello spessore delle pareti addominali arl un canale che gralatamente si allunga e rappresenta il peduucolo dell'allantoide; questo cauale scrve a melterla iu comunicazione colla rescica urinaria e su di esso appunto decorrono

(1) L'ombelico, che rappresenta la cicatrice dell'ancllo ombelicale uel

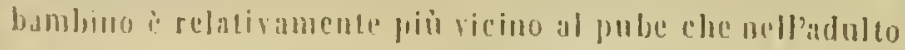


i vasi ombelicali. Il peduncolo prende il nome di uraco, no.* me che conserva anche quando è obliterato e ridotto ad un semplice cordone elastico, che dal vertice della vescica urinaria va all' ombelico facendo le funzioni di un legamento sospensorio della vescica.

In alcuni animali la vescicola allantoide vera acquista grandi dimensioni, dividendosi talvoita in modo da formare due grandi bisacce; allora è mantenuta distesa da molto liquido che ha press' a poco i caralleri dell' urina; lo stesso serve al pari del liquido amniotico a proleggere il feto dagli urti esterni e, per gli animali multipari, anche dalle scosse che potrebbe ricevere dagli altri embrioni della stessa gestazione; in questi animali l'allantoide accoglie dapprima il prodotto dei corpi del Wolf ed in seguito quello dei reni permanenti; e perciò l'uraco si oblitera dopo la nascita. Nell'uomo invece pare che l'allantoide esterna ed il suo peduncolo si obliterino una volta che il fondo di quella vescicola abbia raggiunto il chorion in corrispondenza della decidua serotina, e perciò da questo momento i vasi della rete dell'allantoide colle loro anse terminali penetrano nei villi del chorion e cosi vengono a trovarsi in mediato contalto con quelle dei vasi delle pareti dell' utero. Le prime nel loro insicme costituiscono la placenta felale, le scconde la placenta materna.

Da quanto si disse risulta chiaramente che i due rami terminali primitivi dell' aorta debbono allungarsi in proporzione dello sviluppo dell'allantoide e del suo peduncolo, ed il loro tratto contenulo nella cavità addominale decorrerà ai lati della vescica urinaria, descrivendo un arco convesso in basso convergente con quello del lato opposto verso l'ombelico, donde escono col peduncolo dell' allantoide per decorrere su questo e raggiungere la placenta fetale; ciò che si disse delle arterie vale anche per la vena o per le vene che riconducono il sangue al felo. (1)

(1) Quando la placenta trovasi situata rerso il collo dell' utero, ció che forma un ostacolo al parto, dicesi placenta pravia. 
Questi tre pasi (1) uniti insicme costiluiscono il funicello ombelicale, clie si allunga gradatamente coll'allontanarsi del feto dal punto dell'utcro ove s'cra fissato l'ovulo, ed all'epoca della uascita ha raggiunto la lungherza di circa un metro. I tre suindicati rasi non hanno un decorso rettilineo e parallelo, ma sono avvolti a spirale ordinariamente da destra a sinistra e le arterie intorno alla vena. Sono tenuti insieme da un particolare tessuto mucoso gelatinoso detto gelalina del Warlon; nello spessore del funicolo si riscontrano inoltre alcuni vasi linfatici, il residuo del dotto omfalo-meseraico ed in fine l'uraco obliterato.

La placenta fetale, come lo indica il suo nome, ha la forma di una focaccia la quale non è interamente circolare; il suo diametro varia fra 5 ed 8 once, lo spessore è di 1-1 $1 / 2$ oncia ed il peso è di 1 a 2 libbre e mezzo ; essa è ordinariamente situata sul fondo dell'utero in vicinanza delló sbocco di una tuba e rivestita dal chorion e dalla decidua riflessa clıe dai suoi margini passano sulle pareti dell' utero, colle quali però non hanno aderenze.

La placenta è un organo assolutamente vascolare perchè i vasi embrionali appena giunti sulla sua faccia fetale si ramificano e si dirigono verso l' utero, continuando a suddividersi in molteplici rami di breve estensione ed intrecciati fittamente fra loro; costituendo in tal modo un parenchima quasi compatto ed elastico, il quale dopo una certa macerazione nell'acqua si presenta come formato da tanti fiocchi o nappe che ripetono in piccolo più o meno la forma della placenta e che perciò hanno un peduncolo, rappresentato da due o tre vasi sanguigni, cioè da una o due arterie ed una vena, una faccia dorsale convessa piuttosto liscia ed una faccia uterina villosa per le anse vascolari sporgenti.

Nella placenta si hanno frequenti anastomosi fra $\mathbf{i}$ rami delle due arterie ombelicali , mentre le anse terminali alla

(1) Non di rado vi hanno due vene ombelicali. 
faccia uterina dei fiocchi rappresentano il passaggio fra le ar terie e le vene ombelicali ; percio inictlando una massa od unl liquido colorato per un arteria si vede ritornare sia per l'altra arteria, sia per la vena. Alcune vene maggiori si uniscono e formano alla faccia fetale della placenta una specie di gran seno periferico.

I fiocchi o lobi della placenta fetale nell'utero gestante sono circondati fino al perluncolo dalla decidua serolina, e siccome il peduncolo corrisponde piì o meno al centro di questi corpi (che hanno la forma di una sfera schiacciata) tutta la placenta resta fissamente saldata contro la parete dell'utero. $\Lambda$ questo mezzo di unione s'aggiunge anche l'involucro formato dalla stessa serotina intorno alle innumerevoli anse vascolari di cui consta ogni fiocco.

Permettendoci ora una similitudine, che crediamo acconcia, la placenta fetale corrisponde al ceppo delle radici di una graminacea, per esempio del grano turco, perchè nanca di una radice principale che si prolunga in basso, ma immediatamente alla base del fusto (funicello ombelicale) si staccano molte radici avventizie (rami dei vasi ombelicali), le quali continuano a ramificarsi in modo da formare nel loro assieme una chioma fitta di ramificazioni.

I germi di questa bella ed importante vegetarione sono rappresentati dai villi della zona pellucida; infatti passando in breve rassegna tutto il processo di formazione della placenta fetale noi vediamo come dapprima l'ovulo si fa villoso per lo sviluppo di appendici sulla membrana vitellina a zona pellucida. Queste villosità sembrano in origine solide e non presentano, come la membrana dalla quale sono sôrte, alcuna struttura. In seguito ogni villo si fa come ramificato per sviluppo di gemme della sua stessa natura. e poi direnta cavo nell'interno, ove vedesi ben presto tap^zzato da cellule del blastoderma che si fecero slrada nel villo primitivo come le dita d'una mano in quelle del gurunto; nell' istesso modo ogni villn o ramificazione di villo si riveste allestermo di allere cellule. 
rioè di quelle della decidua riflessa; piò tardi penetrano nol la cavita dei villi le anse vascolari dell'allantoide ed allora si ha il chorion vascolare. (1) Nell'uomo e negli animali con placenta acquistano grande sviluppo, per forte ramificazione, soltanto i villi del clorion in corrispondenza della placenta, mentre gli altri si atrofizzano e scompaiono.

I villi del chorion secondo Carpenter ed Ercolani nou si fanno strada nelle glandule uterine o nelle lacerazioni risultanti nella mucosa uterina pel flusso meustruo, ma vengouro a póco a pocó ad cssere circondati dalla decidua serotina, la quale fra un villo e l'altro si solleva per lo sviluppo delle sue anse vascolari; da queste anse se ne producono altre e così contiluando si origina come un tessuto alveolare, nelle cui celle o cavilà vengono appunto a penetrare e ad cssere stretti i villi. Le pareti di queste celle sono quindi formate dalle pareti dei vasi sanguigni della mucosa uterina coperte naturalmente da uno strato cellulare epiteliale; e siccome questi vasi si fanno varicosi e sinuosi per l'abbondante afllusso di sangue, cosi ne viene che sempre più si stringono attorno alle radici dei villi sul chorion, rendendone più salde le aderenze. Le anse vascolari della decidua serotina presentano tra loro molteplici comunicazioni, e per lo straordinario dilatamento che soffrono finiscono per non presentare più il tipo vasale, sì nella struttura delle loro pareti, che per l'irregolarità di forma e distribuzione.

Queste cclle clie si devono formare tril le anse varicose della mucosa uterina ed in cui s' introducono i villi, secondo Carpenter ed Ercolani avrebbero il significato di glandule di nluova formazione, nelle quali l'apparecchio vero secretore o modificatore del plasma sanguigno materno sarebbe rappresentato dagli strati cellulari sopra descritti interposti fra le parcti delle anse terminali dei vasi ombelicali e quelle dei seni n varicositi rlei vasi utcrini.

(1) Ter anse vasenlari dej villi rouservaun sempre lo stesso lipo e la me. decima strultura 
La funzione di queste nuove glandule sarebbe quella di preparare dal plasma sanguigno un umore che verrebbe assorbito dai villi del chorion come il clumo dai villi intestinali. $\mathrm{Ci}$ pare un pó esagerato questo modo di considerare le cose, giacchè sebbene rigorosamente non si possa negare il significato di apparecchio modificatore del plasma sanguigno ad ogni cellula e tanto più ad uno strato di cellule, pure non si ha alcun dritto di considerare come vero organo glandulare una semplice membrana tapezzata da uno o più strati di cellule, poichè in tal caso il peritoneo, la cornea, la pleura ed anclie le pareti stesse dei vasi dovrebbero chiamarsi glandule. Sicuramente non si poteva pretendere che le anse vascolari del feto fossero all'intutto scoperte, come noll ve n'è esempio in nessuno organismo animale, e gli strati $b \quad b \quad b \quad b$ che l'Ercolani a Tav. 10 fig. $2^{a}$ ec. vuol chiamare come organo glandulare, non sono altro che gl' involucri naturali dei vasi, cioè il blastoderma, la zona pellucida, la decidua reflexa e la serotina.

Ammesso pure che i rapporti vascolari fra la placenta fetale e la mucosa uterina si stabiliscano come vogliono i sullodati autori, si ha sempre il fatto che il sangue il quale scorre nelle anse terminali della placenta fetale trovasi diviso da quello che circola assai lentamente nei scni della mucosa uterina per mezzo dei seguenti strati :

$1^{\circ}$ pareti dei vasi fetali,

$2^{0}$ strato delle cellule del blastoderma,

$3^{\circ}$ membrana vitellina anista,

$4^{0}$ strato delle cellule della decidua riflessa,

$5^{0}$ strato cellulare epiteliale e pareti delle sinuosità o dilatazioni vascolari della decidua uterina.

Noi pertanto ci troviamo ancora piu disposti a ritenere che lo scambio materiale fra il feto e la madre si verifichi nella placenta precipuamente per processo di diffusione, essendo constatato che non vi ha alcuna diretta comunicazione anastomotica fra le anse vascolari ultime della placenta felale ed i 
seni o varicositi dei vasi della mucosa uterina da cui sono circondati.

Non v' la d' uopo clie d'/una certa esperienza e confidenza colle ricerche di diffusione mediante endosmometri, per non trovare alcuna difficoltà a spiegare il continuo e rapido scambio di materiali fra il sangue del feto e quello della madre per via osmotica. Il primo deve essere naturalmente assai meno denso del secoudo poichè l' acqua organica, quale prodotto d'ossidazione fetale (1), uon può essere eliminata tutta per perspirazione cutanea, per evaporazione pulmonale e per tutte le altre secrezioni ed escrezioni che si compiono nella vita extrauterina. Nè sicuramente si potrà pretendere che il solo liquido amniotico e l'urina contenuta nella vescica urinaria del neonato contengano tutta l'acqua d'ossidazione prodotta dal, feto durante la vita intrauterina, e perciò attraverso delle anse della placenta fetale deve verificarsi un energico ed incessante processo esosmotico di acqua, la quale tiene in soluzione l' acido carbonico e la maggior parte dei prudotti di ossidazione solidi organici e salini. D'altra parte il sangue pseudo-venoso contenuto nei seni della mucosa uterina, si metterà in equilibrio di saturazione quantitativa e qualitativa con quello del feto, cedendogli ossigeno e materiale nutritivo ossidabile.

Tutt'al più agli strati di cellule epiteliali che rappresentano il mezzo divisorio o d'unione tra i vasi fetali e materni, spetterebbe forse soltanto la funzione di modificare, concentrando il plasma essudato dai vasi uterini nell'atto che le attraversa aggiungendovi qualche principio del loro protoplasma.

$E$ tanto più ci confermiamo in quest'idea dal momento che abbiamo osservate delle gravidanze extrauterine addominali, uelle quali i feti si erano ben sviluppati ed avevano raggiunto il termine di maturanza, untrendosi mediante un funicel-

(1) I tessuli e gli organi nei feti sono assai piu ricchi d'acqua che negli adulti. 
lo ombelicale che terminta con ramilieaxioni grossulane dei suoi vasi aggomitolate in un sol corpo, il quale era saldato contro le pareti adlominali da tessuto unitivo riceco di vasi varicosi comunicanti coi vasi normali delle pareti stesse.

Dupo ciò noi non troviamo alcuna ragione per negare come la l'Erculani che lo scambio materiale fra il feto c la madrc avvenga per semplice diffusione, tanto più che questo processo non può esser negato da alcuno per quella parte che riguarda i gas, l'acqua e le sostanze che questa puó temer sciolte, e perchc̀ abbiamo la convinzione che il passaggio delle sostanze veramente alimeutari e digerite anche nell'intestino dell'adulto si verifica per diffusione, mentre i cosi detti fenomeni di assorbiinento intestinale nello strelto senso della parola, cioè pér capillarità, rappresentano appunto la nebbia che ancora offusca l'orizzonte scientifico di questa funzione. Ed è pertanto a desiderarsi che i cultori delle scienze sperimentali si adoperino a diradarla, anzichè giovarsene per farne il manto a nuove teorie forse per coprirne l'incerta base.

GIRCOLAZIONE DEL FETO PRIMA JELLA NASCITA

Il sangue arricchito di materiali di nutrizione e di ossige110 si porta per la vena ombelicale al fegato al quale organo arriva anche il sangue della vena porta. La continuazione della vena ombelicale rappresentata dal dotto di Aranzio sbocca direttamente nella cava ascendente, la quale perciò porta all'atrio destro la maggior parte del sangue reduce dalla placenta, mentre quella piccola porzione di questo stesso sangue, che per le vene adveenti andò ad irrorare il parenchima epatico si versa nella cava ascendente insicme al sangue della vena porta. Per la presenza della valvola d'Eustachio e del tubercolo del Lower nell' atrio destro il sangue che vi viene portato dalla cava inferiore non può discendere nel rentricolo soltoposto ma è condotlo verso l'atrio sinistro, in cui si versa passando pel forame ovale che trovasi dirimpetto allo sloceo della rava 
ascendente 1 ; Quindi questo saigue più nutritivo e piò ossigenalo passa dall'atrio sinistro nel ventricolosottostante, che lo spinge nell'arteria aorta, d'onde scorre nei primi tronchi arteriosi (coronarie, carotidi, vertcbrali e succlavie ) e va pertanto a nutrire il cuore, la testa, gli arti superiori ed il sistema nerroso cerebro-spinale. Il sangue venoso reduce da queste parti, si versa nell' atrio destro per la cava discendente e per la $v$. cardiaca-magna, e non trovando ostacoli a discendere nel rentricolo destıo, viene spinto da questo nell'arteria polmonare; siccome i polmoni prima della nascita non possono dilatarsi e ricevere tutto il sangue dell'arteria pulmonare, cosi il sangue spinto in quest'arteriadal ventricolo destro si fa strada pel dollo del Botallio e passa nell' aorta, ove si mescola col sangue residuo che non prese la via dei tronchi arteriosi dell' aorta esistenti tra il cuore ed il dotto di Botallio; cosi i visceri addominali e gli arti iuferiori ricevono un sangue meno ossigenato e meno nutriente. (2)

Inseguito ai primi movimentirespiratorii o vagiti (che sembrano piuttosto rillessi dalla periferia, cioè provocali dalle nuove ed insolite impressioni cui trovasi esposto il neonato) il sangue spinto dal cuore destro fluisce liberamente nell' arteria polmonare e sue diramazioni, cioè non passa più nell'aorta per la via del dotlo del Botallio, poichè viene come aspirato nei polmoni che si distendono in seguito alla dilatazione della cassa toracica operata dai muscoli inspiralori.

Per necessità quindi le vene polmonari vengono ad essere

(1) La valvula d'Eustachio ha la forma di una falce col margine libero diretio in alto; essa si estende col margine fisso dalla periferia destra dello shocco della cava ascendente od inferiore alla branca anteriore dell' istmo del ricussen o lembo del forame vrale. Il tubercolo del Lower e una sporgenza della parce dell'alrio destro situata fra gli sbocehi delle due vene care.

(2) Da cio si spiega la sproporzione fra gli arti superiori e gl' inferiori nei fetied anche nei neonati in confronto dell'adulto. 
percorse per la prima volta da gran copia di sangue, e nell'unità di tempo ne versano nell' atrio sinistro tanto quanto le vene del corpo ne conducono nell'atrio destro. (1) Come abbiamo detto a pag. 123 si chiude presto il forame ovale e così si compie la divisione totale del cuore in una metà destra o veno$s a$, cosi detta perchè riceve il sangue venoso reduce da tutto il corpo e lo porta al contatto dell'aria atmosferica nei polmoni, ed in una sinistra od arleriosa, che riceve il sangue fatto arterioso néi polmoni e lo spinge in tutto il corpo. I vasi che non vengono piu percorsi dal sangue, quali sono il dotto dell'Aranzio o tratlo della vena ombelicale alla faccia inferiore del fegato, il dolto del Botallio e le arterie ombelicali, si obliterano e si riducono a cordoni più o meno permanenti e riconoscibili anche nell'adulto.

Poche e scarse sono le cognizioni fisico-chimiche sui mutamentidei principii elementari e sul commercio materiale che ha luogo nell'orgallismo gravido tanto col mondo esterno quanto coll' embrione; come pure mancano le cognizioni delle cause fisiologiche del parto, del suo regolare avvenire dopo unacerta duratd della gravidanza, sulla meccanica dell'attivilà dell'utero, sulle vie e sui centri d'innervazione che producono e regolano l'atto del parto.

Si asserisce che il sangue delle donne gravide sia più oscuro, perchè più ricco di acido carbonico, e che contenga maggiori quantità di fibrina, di grassi e di globuli rossi, i quali sarebbero più densi e precipiterebbero più prontamente al fondo, in modo che gli strati superiori del coagulo sarebbero bianchi perchè formati da pura fibrina, come succede nel sangue flogistico.

(1) La vena ombelicale dopo la nascita naturalmente non conduce pit sangue al cuore. 
L'at to del concepimento non si manifesta per segni certi e riconoscibili, sebbene si dica da taluni che le donne le quali hanno figliato piì volte acquistino come un senso speciale clie le avverte se il coito sia stato fecondo oppur no. Noi dubitiamo assai dell'esistenza di questo senso speciale, poichè la virtì di non ingannarsi in fatto di sensazioni ed impressioni è molto rara nell'uomo e specialmente nel sesso debole.

In alcuni trattati d'ostetricia la gravidanza o gestazione viene divisa in tre epoche;

la prima dalla concezione al quinto mese:

quest'epoca viene indicata dalla cessazione della menstruazione; il cessare dei menstrui si ritiene come uno dei segni più certi dell'avvenuta concezione, epperò non è ancora assoluto, essendochè alcune donne continuano ad essere menstruate ad onta che sieno gravide, ed altre, sebbene più rare, non sono mai menstruate nè prima nè dopo la fecondazione. Gli ostetrici e le gravide si servono di questo dato, cioè della prima cessata menstruazione, per calcolare il termine della gravidanza e l'epoca approssimativa del parto; diciamo approssimativa, pnichè quand' anche la donna abbia avuto commercio coll'uomo una sola volta dopo dell'ultima menstruazione, non si può determinare in alcun modo e con precisione il momento della caduta dell'uovo e l'incontro fecnndo di questo collo sperma;

la seconda dal quinto all'ottavo mese :

è contradistinta dalla comparsa dei movimenti del feto avvertiti dalla madre e che possono essere anche constatati dall'ostetrico tenendo applicato l'orecchio o la palma della mano sull'addome della gravida ;

la terza epoca dalloltavo al nomo mese compito :

si conosce per la presenza del feto sul segmento inferiore dell'utero, ciò che si verifica mediante l'esplorazione col dilo introdotto in vagina. 
I fenomeni che presenta la donna durante la gravidanza dipendono dalle modificazioni che subiscono l'utero e gli altri organi dell' economia muliebre. I più notevoli cambiamenti sono naturalmente quelli dell' organo gestante. Essi sono relativi al volume, alla forma, alla strullura, alla situazione, alla direzione, alla mobilità, a' suoi rapporli e proprictà fisionlogiche.

Infatti avvenuta la concezione l'attività di nutrizione degli organi genitali femminili viene diretta alla nutrizione dell'embrione; la mucosa dell'utero, come abbiamo veduto, si ipertrofizza per formare la decidua, ed i vasi sanguigni della mucosa uterina, in cui sboccano i rami arteriosi spirali, formano delle anse le quali s'ingrossano e ne sviluppano altre e finalmente facendosi varicose si mettono in rapporto col chorion frondoso dell' embrione: infine il collo e l'nrificio dell'utero vengono ar essere chiusi per l'inspessirsi delle mucos" e per un tappo di muco che s'intromette nel collo.

E pertanto alla congestione erettiva periodica, che sembra la causa della menstruazione, subentra un turgore continuo che ha per risultato finale l'aumento in volume dell'utero con inspessimento delle sue pareti, prodolto da ingrossamento degli elementi istologici preesistenti e da neoformazione di cellule epiteliali, di connettivo, fibro-cellule muscolari, vasi e fors'anco di nervi.

Pare anche probabile che s' arresti lo sviluppo de' follicoli di Graaf, perchè in un'ovaia vi sarà anemia, e nell'altra la circolazione è impegnata a sostenere il processo infammatorio ed essudativo del corpo luten, il quale dal suo canto comprime lo stroma ed impedisce l'ingrossamento d'altri follicoli.

L' aumeuto in volume dell' utero è piccolo nei primi mesi e considerevole negli ultimi. A gravidanza matura l'altezza dell'utero è di circa quattordici pollici e la larghezza di otto in nove; lo spessore delle pareli di circa mezzo pollice.

La forma dellutero si cambia pure in quanto che da piriforme schiacciato si fa tondeggiante ed infine diventa ovale. 
Cosi pure le due labbra della bocca dell' utero a poco a poco si mettono allo stesso livello e l'orifizio vaginale da trasversale elittico diventa circolare. Nelle primipare l'orifizio è ristretto ed il contorno levigatissimo; ma nella donna già madre la bocca dell'utero è meno ristretta ed il contorno ne è irregolare. Coi cambiamenti di volume e forma vanno di conserva i mutamenti di sito dell'utero. Nei primi tre mesi rimane nell escavazione pelvica col fondo leggermente piegato all' indietro ed a destra ; nel quarto incomincia ad elevarsi al di sopra del distretto superiore del bacino per circa due dita trasverse ; al quinto il fondo dell' utero ha quasi raggiunto il piano dell'ombelico; a sei mesi lo ha sorpassato e finalınente all' ottavo mese corrisponde al limite inferiore della regione ipogastrica;nella prima metà del nono mese continua ad innalzarsi; di poi, cioè nella seconda metà, si abbassa, ciò che pare dipenđere dalla discesa del feto e dall' assottigliamento delle pareti uterine nel segmento inferiore. Coll'innalzarsi dell'utero il fondo piega sempre più in avanti ed a destra per cui il collo si porta posteriormente ed a sinistra.-

La mobilità dell'utero va diminuendo in ragione direlta del suo ingrandimento. La sensibilità invece aumenta colla gravidanza, specialmente nel suo collo.

I citati mutaınenti dell'utero prodotti dalla gestazione non possono andar disgiunti da altri nelle parti vicine ed anche nelle lontane influenzate da quest'organo, come per consenso e simpatia, dando luogo a molti dei così detti segni della gravidanza.

Perl'innalzamento dell'utero le ovaie si inclinano e le trombe faloppiane prendono una direzione pressochè verticale. I legamenti rotondi si allungano e vengono stirati: la vagina pure si allunga, massime negli ultimi mesi, e le glandule della mucosa che la taperza segregano maggior quantità d' umore; anche i genitali esterni diventano sempre più molli e cedevoli; la vescica urinaria viene stirata in sil e compressa dall'utero, dal che viene una piu frequente emissione di uri- 
ne; l'uretra è tumefalta ed ascende piu verticalmente dietro la sinfisi del pube.

Per la compressione esercitata dall'utero ingrossato sui visceri, sui vasi sanguigni ed anchesulle pareti addominali, che restano ostacolate nei loro movimenti, ma più specialmentesul diaframma, si spieganotanti fatti assai frequenti nelle gravide, quali sono; la stitichezzza, i dolori addominali, la difficile respirazione, l'edema e le varicosità degli arti inferiori.

Ia pelle dell'addome negli ultimi tempi della gravidanza è oltremodo distesa, specialmente verso la parte inferiore, ove presenta delle macchie biancastre; i muscoli si distendono e si assottigliano, la linea alba si allarga considerevolmente, massime dall' ombilico al pube; la depressione ombilicale scompare a poco a poco, facendosi negli ultimi tempi I' ombilico alquanto prominente.

Il rilassamento della sinfisi del pube è un fenomeno costante della gravidanza,per cui nel momento del parto le ossa del bacino si riscontrano sempre più mobili di quanto ordinariamente non sono.

Le mammelle infine, che possono essere considerate una dipendenza degli organi genitali, subiscono anch'esse notabili modificazioni, che le dispongono alla funzione cui esse sono destinate dopo il parto. Nella gravidanza questi due organi si tendono e gonfiano e secernono anche un umore lattiginoso; il capezzolo si pronuncia maggiormente e l'areola si allarga facendosi più bruna.

La gravidanza si riconosce dalla presenza di alcuni segni, i quali si distinguono in presuntivi e cerli. I primi vengono costituiti da certi fenomeni che si osservano nella donna e che diconsi simpalici. Gli occhi diventano languidi; sotto della palpebra inferiore si presenta un semicerchio oscuro (occhiaia); il naso si fa affilato, gli aıgoli della bocca vengono stirati all'indietro, il mento si rende prominente, il volto tint to impallidisce e presenta talora delle macchie; il collo si gonfia, le mammolle ingrossano, i polsi si fanno fre- 
quenti, il calore della pelle aumenta; sorgono non di rado appetiti speciali e talvolta depravati, inappetenza, salivazione, nausea, rounito, vertigini, dolori intestinali e stanchezza generale; il morale della donna è più o meno alterato, le facoltả intellettuali talora si sviluppano, talora si ottundono; le urine si cambiano, sono spesso torbide, alcaline e facilmente si forma alla loro superficie una pellicola con cristalli di fosfato doppio d'ammoniaca e magnesia (1). Tutti questi segni sono per sè stessi poco concludenti. I dubbi che essi lasciano vengono dissipati dalla presenza dei segni cerli, i quali si rilevano col mezzo dell'esplorazione e dell'ascoltazione é consistono nei movimenti attivi e passivi del feto e nei battiti cardiaci dello stesso.

L'esplorazione consiste nell' esame fatto colle dita o colla mano portata nella vulva, nella vagina, nel retto o sopra l'addome.

L'ascollazione consiste nell' applicazione immediata o mediata dell' orecchio sul ventre della donna incinta per sentire le pulsazioni del cuore del feto.

Mediante l'esplorazione si scoprono i movimenti attivi e passivi del feto. I primi si manifestano ordinariamente nel quinto mese, qualche volta prima, altre volte più tardi; leggerissimi dapprincipio vanno facendosi sempre più distinti e forti fine al termine della gravidanza. Dipendono essi dall' urtare del feto contro le pareti dell'utero, ne' vari movimenti che eseguisce. L' applicazione delle mani fredde sul ventre della gravida e lo scuotimento dell' utero, sono i mezzi migliori per risvegliarli. I movimenti passivi sono quelli che vengono impressi al feto, o dalle variate posizioni della gravida, o per opera dell'esploratore, che introducendo il dito indice nella vagina fino a raggiungere la parete anteriore 0 posteriore dell'utero, lo urta in modo da innalzare il feto che

(1) Ciò dipende probabilmentc dall'abbondanza di muco che ne favorisce l'alterazione alcalinis. 
poscia ricade con forza e percuote il dito : questi movimenti si dicono di ballottamento o di rimbalzo.

Questo moto non si può ottenere ben distinto se non dopo il sesto mese di gravidauza. I morimenti allivi come è cosa naturale si hanno solamente quando il feto è vivente, mentre i passivi si possono avere anche da un felo morto.

Applicando l'orecchio sul ventre della donna gestante, od ascoltando col mezzo dello steloscopio, dal quarto mese della gravidanza in avanti, in un punto non determinato, sentesi un baltito molto frequente a doppio suono, il quale è prodotto dalle pulsazioni del cuore del feto. Questo battito distinguesi facilmente da quello dipendente dalla circolazione uterina, per essere il secoudo meno frequente, semplice, ed accompagnato da un soffio particolare detto placentare.

ATTEGGLAMENTO E POSIZIONE DEL FETO NELL'UTERO

Il feto maturo contenuto nell' utero rappresenta un corpo ovoide, nel quale si distinguono due estremità, una delle quali grossa, molle, ollusa formata dalle natiche od estremilà pelvica; l'altra meno grossa, dura, meno oftusa costituita dalla lesta.

Tale formạ ovoide risulta per l'atteggiamento proprio del felo, che ha la testa piegata ed apjoggiata col mento sullo sterno, i piedi in flessinne dorsale sulle gambe e piegati all'interno, le gambe forlemente flesse e picgate sulle coscie, le quali stanno applicate contro l'addome, coi ginocchi alquanto distanti, poichè le gambe sono incrocicchiate sicchè il tallone destro tocca la natica sinistra e viceversa; le braccia sono adattate alle parcti laterali del torace, sli avanbracci sono flessi ed incrocicchiati sullo sterno; le mani fatte a pugno appoggiano sulle guancie. Questo alteggiamento pel quale il feto occupa il minore spazio possibile sembra che non dipenda dalla pressione esercitata dall utero ; infatti il neonato abbandonato a sè stesso su di un soffice lello prente 
spontaneamente più o meno lo stesso atteggiamento, divenuto abituale e forse voluto dalle condizioni meccaniclıe (elasticita) delle masse muscolari, degli arti e del tronco.

Pare che prima del settimo mese il felo non abbia una posizione fissa; da quest'epoca innanzi, forse per legge di equilibrio, si colloca colla testa in basso verso la bocca dell'utero e colle natiche in alto; il che fayorisce anche la sua uscita, come vedremo nel capitolo seguente.

La durata normale della gravidanza è di circa duecento ot tanta giorni, cioè di dieci mesi lunari o nove solari. Questo termine può essere oltrepassato (1), oppure la durata della gravidanza puó essere più breve di alcuni giorni o settimane senza che il neonato offra caratteri spiccati d'immaturità 0 di troppo progredito sviluppo. (2)

Se il parto avviene dopo il settimo mese solare, il neonato può vivere; per altro, astrazione fatta anche dalla piccolezza, sotto più rapporti può dirsi immaturo e la mortalità dei nati a sette mesi è maggiore di quelli che raggiungono il termine naturale. Per quanto è a mia cognizione sono più freqnenti i parti a sette mesi che quelli ad otto mesi.

D'ordinario i difetti dipendenti da parto prematuro non si correggono mai e si hanno degli uomini piccoli, cachettici, con vizii cardiaci ecc. Diversi individui di mia conoscenza nati a sette mesi presentano vizii cardiaci, ed alcuni fra questi sono figli a genitori che soffrono difetti analoghi.

La periodicita delle menstruazioni ha un riscontro coll' epoca del parto, imperciocchè quando è normale suole avvenire allorchè si dovrebbe manifestare la $10^{\mathrm{a}}$ menstrua= zione dopo la concezione, e gli aborti come i parti premalu-

(1) Qualche volta il parto ritardato è soltanto appareute per essere avyenuta la fecondazione un certo tempo dopo del coito.

(2) Nelle primipare il parto si anticipa t'ordinario di alcuni giorni. 
ri arvengono, nel maggion numero dei casi, in epuche anjoghe, cioè i primi nell' $8^{\mathrm{a}}$ nella $12^{\mathrm{a}}, 16^{\mathrm{a}} 0 \mathbf{2 0}^{\mathrm{a}}$ seltimana, gli altri all'epoca dell' $8^{a} \cdot 9^{a}$ menstruazioue.

Gi sono ignote le cause che determinario il fine della gestazione e che eccitano l'utero a vuotarsi. É certo però che non è la presenza del feto, poichè l'utero si contrae e le doglie si verificano anche nei casi di gravidanza extra-uterina, quando cioc̀ il feto non agisce colla sua presenza e coi suoi movimenti come stimolo.

Il parto avviene per contrazioni ricorrenti dell'utero, le quali sono da principio poco intense e di minore durata, ma in seguito diventano più forti e sono accompagnate da dolori e perciò prendono il nome di doglie (1).

I muscoli delle pareti addominali ed il diaframma coadiuvano in prima linea l'utero nella funzione del parto, precisamente nello stesso modo come aiutano la muscolatura dell'intestino nella defecazione. D'ordiuario nella donna vi si associa l'azione di altri muscoli e specialmente quella degli arti superiori, che si contraggono quasi convulsivamente per afferrare oggetti fissi circostanti e farne punto d'appoggio, e quella dei muscoli degli arti inferiori che si piegano ad arco, perchè il corpo possa trovare maggior resistenza, puntellandosi sui talloni e sul sacro.

Per le contrazioni ritmiche, crescenti in forza e durata, e procerlenti dal fondo verso il collo dell'utero, il feto ed il liquido amniotico ancora involti dalle membrane dell'wovo vengono spinti contro il segmento iuferiore dell'utero assottigliato; allora il collo dell'utero si accorcia di più e la bocca si dilata gradatamente.

Continuando le contrazioni uterine il liquido amniotico si fa strada pel primo fuori della bocca dell'utero, scorrendo fra la periferia della testa del felo ed i margini dell' orificio interno uterino. Naturalmente questo liquido spingerà innanzi

(1) Le doglie si ascrivono alla forte tensione della muscolatura uterina. 
a se le membrane dell' novo e perció alla bocca dell' utero si va formando una vescica, la quale s'ingrossa, protrude in vagina ed a modo di cuneo dilata sempre più l'orificio nterino. Le pareti di questa vescica sono formate dalla decidua, dal chorion e dall'amnios. Gli ostetrici la chiamano il sacco delle acque, il quale nou resiste ordinariamente alla continuata e crescente tensione e scoppia lasciando scorrere il liquido contenuto. (1) Intanto l'estremità cefalica (più di rado la pelvica) del feto si è impegnata nell'orificio uterino ed in seguito ad una forle e duratura contrazione viene spinta attraverso la vagina verso il perineo.

Finalmente sotto l'azione di altre doglie che sono appunto le piu intense e conquassanti si determina l'uscita della lesta del feto: dopo un riposo di pochi istanti seguono altre doglie per le quali vengono spinte fuori le spalle, l'una dopo dell'altra, ed in seguito tutto il resto del corpo del feto, che perde ogni rapporto colla madre, quando il cordone ombelicale viene lacerato o reciso.

L'uscita di un corpo si voluminoso relativamente alle vie che è obbligato di attraversare è resa possibile :

$1^{0}$ dalla mobilità delle ossa del pube nella loro sinfisi, che è un carattere del bacino femminile e che si aumenta durante la gravidanza.

$2^{\circ}$ dalla cedevolezza delle parti del corpo del feto e specialmente dalla presenza dellc fontanelle nel cranio, che permettono l'avvicinamento e perfino l'accavallarnento delle ossa craniche, dando alla testa del bambino diametri minori ed una forma temporariamente più opportuna.

Uscito il feto, l'utero si vuota delle acque clic erano rimaste indietro; e da ultimo sotto altre contrazioni uterine meno violente viene espulsa la placenta.

Siccome pel distacco della placenta vengono lacerati alcuni vasi uterini, cosi si ha d'ordinario una emorragia, la quale poi

(1) Non è però raro il caso che il sacco non ci rompa co il felo csea dall'utern involto nelle sue membrane. 
ressa perchè le contrazioni delle pareti muscolari chiudono i lumi dei vasi. L'emorragia alla sua volta fa diminuire il turgore dell' utero. La mucosa dell' utero ritorna allo stato primitivo nel corso di alcune settimane: in questo tempo si osserva un flusso dai genitali mulicbri sanguinolento, indi mucoso ed infine sieroso (lochii). Le fibre muscolari dell'utero diminuiscono di numero e volume; la scomparsa di molti elementi muscolari pare dovuta ad una degenerazionc adiposa degli stessi seguita da assorbimento.

Il parto ha una durata molto varia, da mezz' ora fino a tre giorni ; come pure sono diversamente intensi i dolori che accompagnano le contrazioni uterine ed il consccutivo abbattimento della puerpera. Tutlo ciò dipende principalmente dal grado di robustezza generale della donna, dalla conformazione del bacino, dai rapporti nelle dimensioni reciproche dei genitori e finalmente dalle abitudini della vita domestica. Cosi si spiega come alcune donne partoriscano con facilità sorprendente e quasi senza abbattimento puerperale, al punto da poler continuare le occupazioñi ordinarie che furono sospese momentaneamente dall'atto del parto; mentre altre madri talvolta soccombono alla gravezza di quest'atto per se stesso fisiologico.

\section{NEONATO E LATTAZIONE}

Il nconato è d'ordinario rosso in futta la superficie del corpo e cianotico in volto. Questo coloramento dipende dall' abbondanza di sangue venoso e dalla congestione alla testa, perchè durante il parto la circolazione ombclicale resta impedita e la respirazione polmonare non si può effeltuare fincliè la testa del felo non trovasi in contatto dell' aria e la cassa toracica è impedita a dilatarsi.

La superficie del corpo del neonato c̀ coperta da una patina della vernice caseosa, formata da sostanza grassa interposta a molle cellule epidermoidali che si sono slaccate a strati. Quc. 
sta vernice od intonaco sebaceo protegge i tegumenti dalla macerazione nel liquido aminiolico e ne impedisce I'assorbimento. Il grasso o sebo è sicuramente il prodotto delle glandule sebacee.

II canale intestinale, massime il grosso, è ripieno d'una poltiglia verdastra-oscura detta meconio, clıe è costituitada cellule epiteliali e dai succhi delle glandule dell'apparecchio digerente, specialmente da molta bile da cui dipende il colore verde. La cistifellea è piena di bile, la vescica urinaria è distesa dall'urina; le glandule mammarie contengono un umore lattiginoso (latte di strega).

Nei primi giorni il bambino dorme molto, cioè da 20 a 22 ore al giorno; nelle prime 24 ore d'ordinario non succhia; stante le forti perdite che subisce il neonato nei primi giorni, sia per l'eliminazione del meconio e delle urine come per la respirazione e perspirazione, non compensate dall'alimentazione, diminuisce di peso e d'ordinario nei primi otto giorni dimagra.

La donua e la femmina dei mammiferi non compie col parto la missione clie le spetta per la riproduzione della specie, ma deve per un certo tempo fornire al neonato od ai neonati il materiale di alimentazione (lalle) preparandolo nel proprio organismo e precisamente nelle glandule mammarie, le quali durante la gravidanza acquistano il loro massimo sviluppo.

Durante l'allattamento, che si continua per un anno incirca, la donna d'ordinario non è menstruata ed i coiti sono difficilmente fecondi.

Non è qui il luogo di entrare nei particolari della struttura delle glandule mammarie e del modo con cui secernono il latte, nc̀ della cemposizione di quest' ullimo. Soltanto diremo che l' umore segregato dalle stesse muta caratteri e composizione in progresso dell'allatlamento, e le qualita dell' umore che esce dalla glandula nei primi giorni dopo il parto sono lali da merilargli il nome speriale di colostro (reggasi a pro- 
posito la mia Guida pag. 191) il quale per essere multo denso, grasso e ricco di zucchero scrve, secondo gli ostetrici, piuttosto come di purga per la evacuazione del meconio, anzichè di alimento.

In prosieguo il latte acquista i caratteri specifici e normali contenendo nelle proporzioni piu opportume tutt' i principii organici ed inorganici indispensabili a renderlo un alimento composto perfetto, clie il bambino introduce nel suo organismo in tale quantità che basta mon solo a riparare le perdite di materia e di forze che subisce, ma anche al suo incremento e sviluppo.

Pare che la comparsa dei denti segni il limite naturale al periodo di lattazione, sia perchè allora la bocca del bambino non più si adatta bene alla suzione, come anche perchè si trova munita degli organi passivi necessarii alla maslicazione dell' alimento solido.

Evidentemente prosperano molto bene i bambini che a questa età alternano col latte altri alimenti, specialmente cereali sotto varip forme, cioè paste, riso, pappe, pane ec. la cui digestione è favorita dall' abbondante secrezione di saliva clıe accompagna appunto la dentizione.

La necessitì di supplire alle madri sia perchè mancanti di latte, sia per sventure domesticlie o pubbliche ha falto ricorrere all'allattamento mercenario od artificiale.

Siccome nessun'animale domestico fornisce un latte identico a quello della donna e siccome sembra impossibile di preparare con latte di bruto ed altre sostanze aggiunte un composto che corrisponda perfettamente al latte muliebre, sembrerebbe a primo aspetto che fosse preferibile l'allat tamento inercenario all'artificiale. Ma considerando tulto dal punto di vista dei bisogni generali del ıeonato per la sua educazione fisica e morale, che incominciano dalla culla e s'influiscono a vicenda, non si saprebbe dire qual madre sia più commenderole o riprorerole fra quella che si separa dal suo figlio appena che lıa veduto la luce, affidandolo per molli mesi alle c'ure d'una nutrice merce- 
naria lonlania, e quella clie non abbandona il suo nato ma premurosa lo alleva mediante il poppatoio con latte di giovenca, di capra, ec. cui aggiunge, per correggerne i difetti o gli eecessi acqua, zucchero, brodo, farina, biscotto gratuggiato, acqua di calce, bicarbonato di soda ec. ec. Ed a questo proposito dirò come in Germania ma specialmente in Inghilterrá ed Olanda, forse per l'eccessice esigenze delle nutrici, sieno stati fatti molti tentativi e studii per trovare il migliore succedaneo del latte di donna; e per ciò si sottopose ad accurate analisi comparative il latte della donna, della giovenca, della capra, dell'asina e della giumenta. Come in tutte le altre questioni della vita sociale la preferenza dala ál latte dell'uno o dell' altro animale e quindi anche la scelta delle più o meno complicate ricette per ridurlo idoneo, restò subordinata fino ad un certo punto alla questione finanziaria; cosi vediamo clie mentre nel mezzodi d'Italia si usa e s' abusa di latte d'asina, in Germania ed in Inghilterra non se ne parla punto, essendo questo animale rarissimo ed anzi un oggetto piuttosto di curiosità per quei paesi.

Da miei studi analitici sul latte della donna e di vari animali ho potuto convincermi, dietro ripetuti esami chimici micro e macroscopici, che siamo ben lungi dall'avere una cognizione tale della composizione del latte da poterne con sicurezza affermare i rapporti qualitativi e quantitativi dei suoi componenti, tanto più che esso presenta grandissime variazioni da donna a donna, e nella stessa muta secondo le diverse epoche dell'allattamento e col mutare degli stati fisici e perfino psichici.

Nei bruti poi la qualità e la quantità dell'alimento o foraggio modifica prontamente la composizione del latte e perciò nell' allattamento artificiale si fa uso più comunemente di quello di vacca ; e ciò sia per ragioni economiche sia perclıè questo animale riceve d'ordiuario un foraggio meno vario.

Siccome perí il latte di vacca conticne un eccesso di caseina, ció clıe lo rende meno digeribile al bambino, si allunga 
r'0u acqua: l'aggiuntit peró dell' acqua mentre diluisce il láte rispetto alla caseina lo rende troppo povero di zucchicro e di sali, ed è per questo che alcuni consigliano di allungarlo con brodo di carne, oppure con una soluzione più o meno concentrata di zucchero di latte. $\Lambda$ correggere poi il difetto delle sostanze inorganiclı, inevitabile colla diluzione, e cousiderando che fra queste la calce è la più importante, vuolsi clie in Inghilterra abbiano dato buoni risultati le seguenti formole di preparare il latte di vacca per renderlo confacente al bambino nelle diverse età:

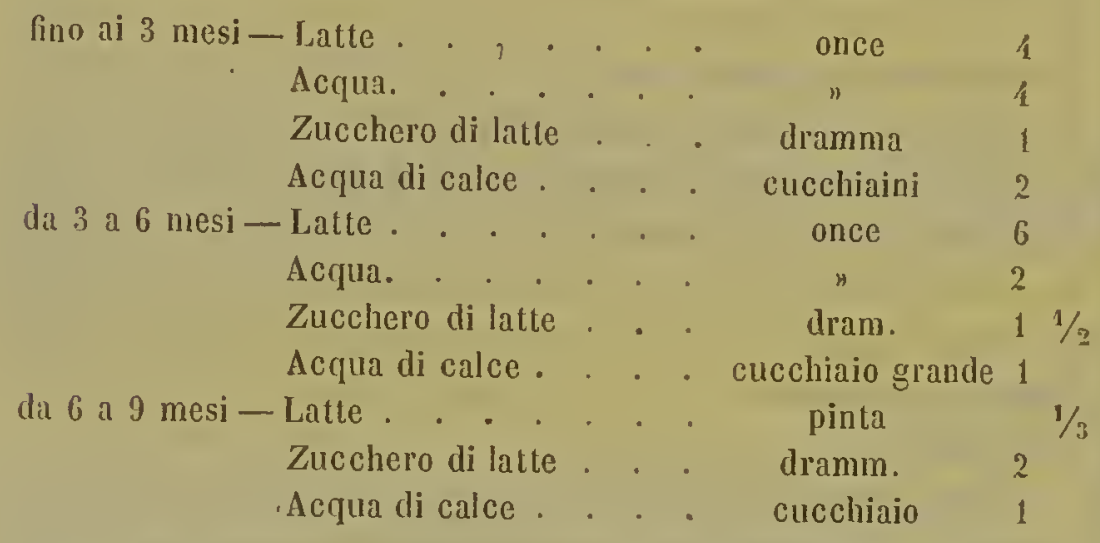

Ciò che importa precipuamente è la prosperità del bambino, cui soltanto possono provvedere le cure solerti dei genitori i quali avendone sotto gli occhi lo sviluppo possono giudicare delle migliori opportunità ; sarà perciò sempre riprovevole l'abbandono alle nutrici lontane e mercenarie, delle quali ancor adesso in alcune provincie d' Italia si fa abuso e specialmente dalla classe colta della società, ove più di una madre dovrebbe arrossire ricordandosi che i suoi figli

Che le alleviaro il dilicato fianco
Non la rivider più; d’ignobil petto
Esaurirono i vasi; e la ricolma
Nilidezza serbaro al sen materno.

FINE DELLA 3. " PARTE 


\section{ATIAANTH}

PEL

\section{TRATTATO DLLLA VITA RIPPODUTTIIIA}

TERZA PARTE

DELLA

\section{GUIDA TE0RICO PRATICA}

A L L O S T UDIO

DELLA

FISIOLOGIA NORMALE E SPERIMENTALE

DEI,

\section{D. ${ }^{r}$ GIUSEPPE ALBIMI}

Professore di Fisiologia nella R." Università di Napoli

\section{A P () L I}

STABII,IMFNTO TIPOGRAFICO VITAIAE

4 - Strada Pisanelli a Regina Coeli

1868 



\section{TAVOLA P R I II A}


ESEMP! WI HIPTODUZIONE DEGLI ANIMALI PLK SCISSIONE E PER GEMME

Fig. 1 - Paramecium chrysalis - individuo semplice.

"2 - Lo stesso in istato di divisione trasversale.

) 5 - Lo stesso in istato di divisione longitudinale.

" 4 - Vorticella microstoma - individuo semplice.

15 e 6 - Lo stesso che si scinde progressivamente dalla bocca all'estremo inferiore del corpo.

„7 - Campanularia volubilis - larva cigliata libera.

" 8 - La stessa fissata.

1) 9 - La stessa che incomincia a produrre la colonia.

„ 10 - La stessa molto sviluppata e che in a presenta gia una gemma.

"11 - La stessa a perfetto sviluppo con polipi contenuti nelle campanelle a'.

1) 12 - Myrianide fasciata che in $a, b, c, d$ presenta delle gemme prossime a staccarsi per costituire altrettanti individui liberi.

"15 - Hydra fusca - fissata ad uno sterpo; a inizio d' una gemma nel punto ove si sviluppa normalmente; $b$ gemma in cui già cominciano a comparire le braccia; c gemma prossima a staccarsi dalla madre. 
T'is. I.
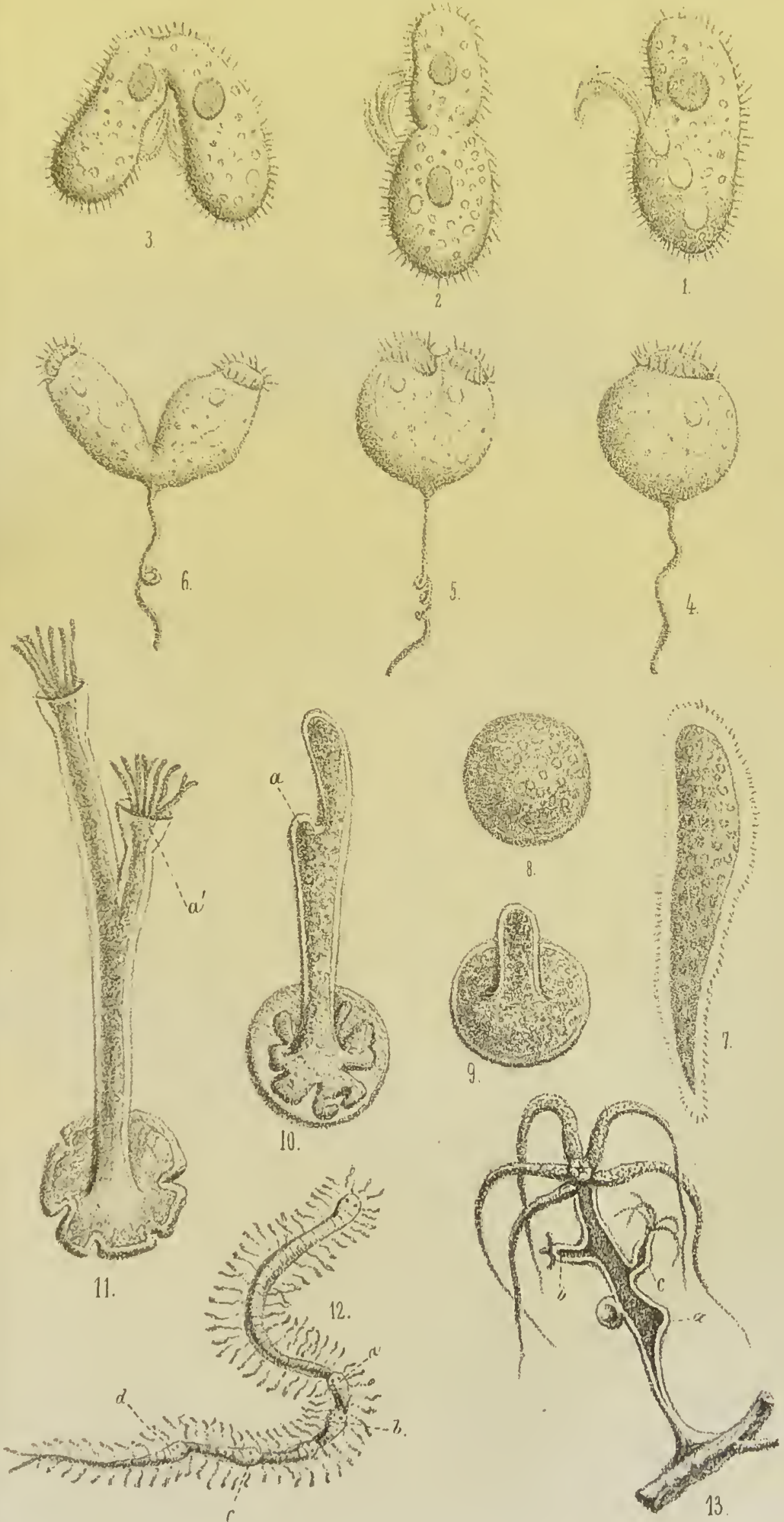

T A V OLA S E C O N D A 
ESEMPI DI RIPRODUZIONE DEGLI ANIMALI PER SPORK. ZIGOSI ED ESIT DELLA STESSA. (1) PEDOGENESI (2)

Fig. 14 - Vorticella microstoma di fresco incistidata.

15 - La stessa nella quale il nucleo si risolve in spore

1) 16 - La stessa colla prole $c$ già sviluppata, che nella figura seguente per rottura della cisti esce ravvolta in una sostanza gelatinosa.

D) 17 - A nuova prole; $b$ sacco degli embrioni rimasto vuoto.

18 - Gregarina libera e vivente.

D 19 - Due gregarine che si congiungono.

) 20 - Le stesse più strettamente unite.

1) 21 - Le stesse inviluppate in una membrana di nuova formazione o cistide comune, con raggruppamenti nel protoplasma.

1) 22 - Prima formazione dei germi o navicelle.

" 25 - Navicelle già fọrmate.

1) 24 - Scomparsa delle due vesciche incluse, rimanendo cosi solo e completamente formato il sacco delle navicelle.

) 25 - Cecidomya allo stato larvale contenente altre larve.

1) 26 - Larva della stessa ingrandita.

1) 27 - Pseudo-ovario con pseudo-ova a diverso sviluppo.

"28-Pseudo-ovo ingrandito.

(1) Per Zigosi s'intende quel particolar modo d'accoppiamento di taluoı unfusori, per cui due di essi dclla medesima specie, e più di rado tre o quattro, si avvicinano si congiungono e finiscono per saldarsi l' un l'altro, secernendo il piủ delle volte una sostanza plastica che condensandosi forma le pareti di una cistide comune. Fig. ${ }^{\circ} \mathbf{1 9 - 2 4}$

(2) Per Pedogenesi s'intende la facoltà che hanoo alcuni animali di $\mathbf{r}_{1}$ prodursi per pseudo-uova essendo aucora allo stato larvale. Le pselldo-tloya o spore, non hauno d'uopo di una fecoudazione. Fig. $25-28$. 

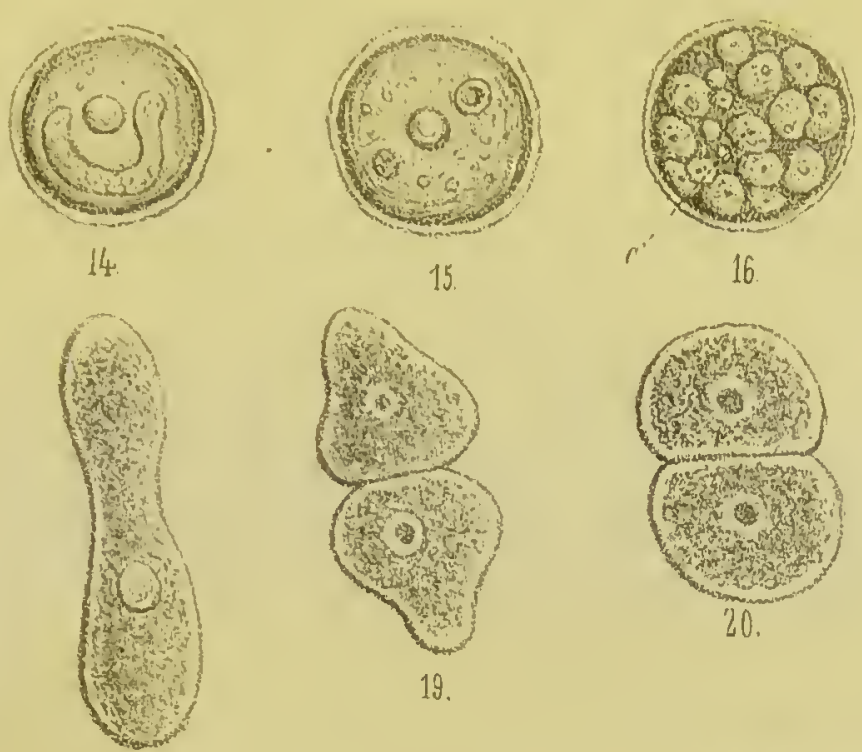

19.
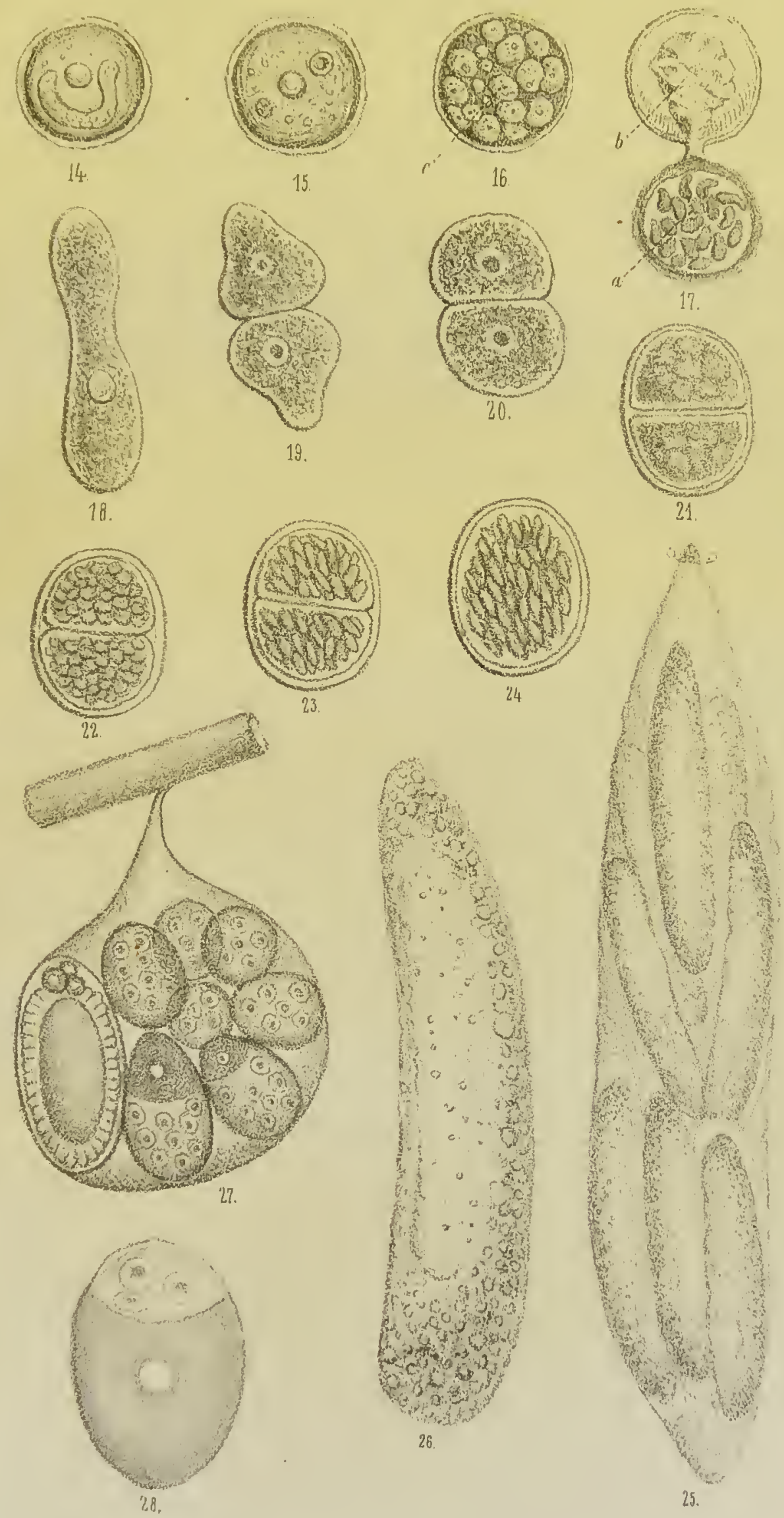

25. 

TA V OLA TE R Z A 


\section{ESFMII DI ERMAFRODITISHO.}

Fig.29 - Taenia echinococcus - individuo intero.

" 50 - Lostesso - Organi genitali nella proglottide ingran. dita; a testicolo; $b$ pene; $c$ c c oraie.

" 51 - Hyrudo officinalis - organi sessuali; $a$ testicoli; $b$ condotti deferenti ; $c$ c vescicole seminali; $d$ borsa da cui diparte il pene; $e$ e ovaie; / utero; $g$ vagina.

" 32 - Glandula ermafrodita della lumaca; $\mathbf{A}$ la glandula: $a$ ovidotto; $b$ canale deferente. B Follicolo della glandula ingrandito; a a a uova a diversi gradi di sviluppo; $b$ filamenti spermatici e cellule che li producono.

" 55 - Rhodope-Glandula ermafrodilica; $a$ a a acini femminili; $b$ acini maschili.

\section{APPARECCHIO GENITALE FEMMINLE NEGII UCCELII}

" 54 - Figura semischematica dell' apparecchio genitale femminile negli uccelli. $O$ ovaia con uova a diversi gradi di sviluppo; $u$ uovo prossimo a cadere, in cui si distingue lo stigma $s$ e la cicatricola $c$; $c a$ calice vuoto ; $O v$ ovidotto disposto ad anse tenute strette in un pacco da un legamento peritoneale ; $t r$ tromba con margine fimbriato aperto sotto l'uovo $u$; $S p$ spaccato dell'ultima porzione dell'oridulto con un uovo contenuto nel quale si distinguono; la latebra 1; la cicatricola 2; il tuorlo 5; le calaze 4: I' albume stratificato 5 ; la membrana testacea 6 formata da due foglietti che al polo ottuso sono staccati l'uno dall'altro e comprendono la camera d'aria 7; il guscio 8 ; $\mathrm{Cl}$ cloaca.

$55-$ Tuorli di gallina; $A$ non fecondato e $B$ fecondato.

1) 36 - Ovaic di un pulcino di pochi giorni, in cui già si vede che la destra sta per alrofizzare. 


\section{'Tav. III.}
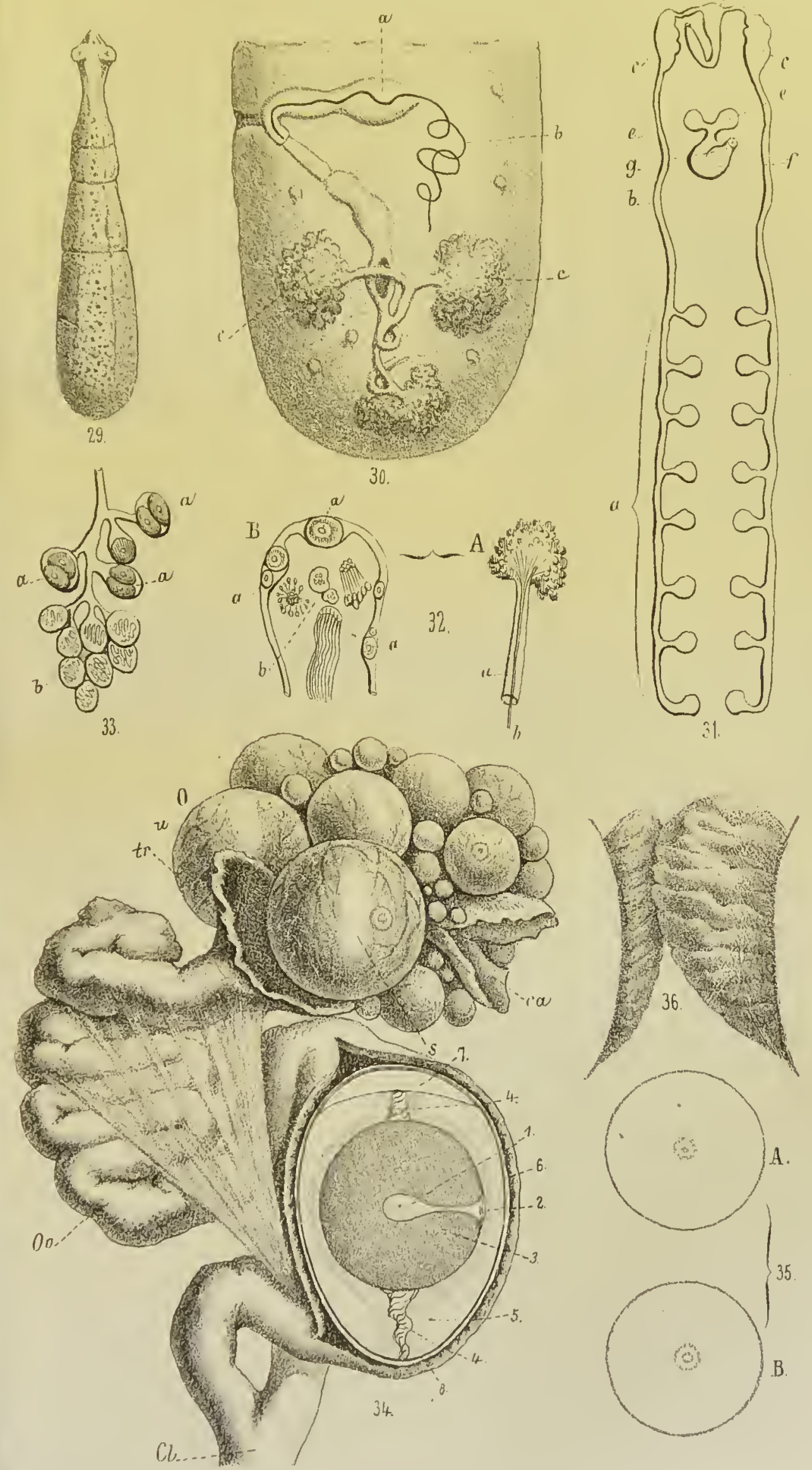

T A V O L A O U A R T A 


\title{
TAVIIA QUATISA
}

\author{
ORGANI GENITALI DELL'UONO
}

Fiy. 57 - Testicolo ed epididimo spogliati dell'albuginea. - II testicolo mostrasi cosi quale aggregato di molti lobuli piriformi $l l$, formali dai tubulin i seminiferi scoverti, che si scaricano per la rete dell'Haller $r$ entro i lobi tubulosi dell'epididimo; $d$ dotto deferente; $v$ vaso aberrante; $e$ epididimo formato dalla riunione dei coni dell' Haller.

" 58 - Spaccato del testicolo per mostrare lacontinuazione dei tubuli seminiferi $l l$ nei tubi dell' epididimo $t$ at traverso al corpod'Highmoro $c$; vg tunica vaginale.

39 - Topografia dell'apparecchio genito-urinario; $p$ pube; $v$ vescica; $u$ uretere; $s$ uretra; $c$ corpi cavernosi del pene; $t$ testicolo; $f$ cordone spermatico; $a$ vasi e nervi; $d$ deferente; $x$ vescichetta spermatica; $y$ prostata.

1 40 - Vescichetta spermatica svolta.

" 41 - Fondo della vescica $V$ a cui fa seguito l'uretra $U$ aperta, essendo incisi nella linea mediana dorsale la prostata $p$, il muscolo pubio-uretrale $p u$ ed il pene $\boldsymbol{P}$. In corrispondenza dello spaccato della prostata si vede nelluretra prostatica il Veru montanum $v m$, ove trovansi tre orifizj, uno mediano od or ifizio dell' utero mascolino, e due laterali che sono gli sbocchi dei dotti eiaculatori. Ai lati dell'uretra spugnosa si veggono i due corpi cavernosi spaccati $c c$; $v$ vasi che decorrono nel tessulo spugnoso; $g$ glandule del Cooper; $e, m$. elevatore dell'ano; $b$, m. muscolo bulbo-cavernoso; $t$. m, trasierso profondo; $s$ sbocchi delle glandule del Cooper; $u r$ sbocchi degli ureteri in vescica.

1) $42-45-44-45-$ Spaccati trasversi del pene; 42 alla radice; 4.3 nel mezzo; 4 . alla base del glonde; 45 all'apice dello stesso. 
Tir. IV.

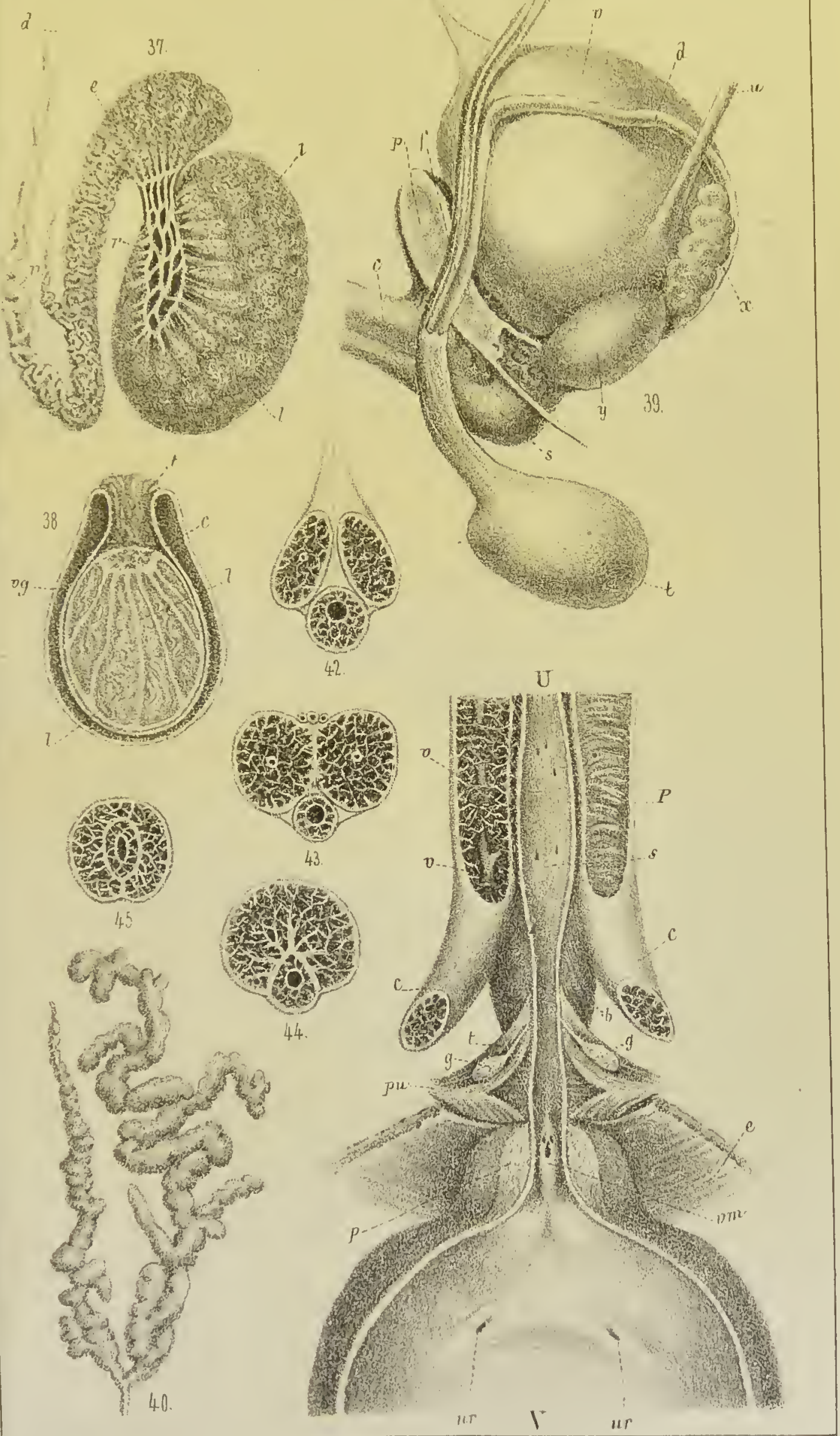



TA V O L A O U I N T A 
ORGANI GENITALI MASCIIII

Fig. 46 - Muscoli esterni dell'apparecchio genitale dell'uomo; $a, \mathrm{~m}$. ischio-cavernoso; $b, \mathrm{~m}$. bulbo-cavernoso ; $t$, $\mathrm{m}$. trasverso del perineo; $c, \mathrm{~m}$. costrittore dell'ano; $e, \mathrm{~m}$. elevatore dell' ano.

1) 47 - Parete anteriore del bacino colla radice del pene, veduta quasi di profilo; $s$ sinfisi del pube; $v$ vena dorsale del pene; $a$ arteria dorsale del pene; $l$ legamento med. sospensorio del pene ; $c$ corpo cavernoso del pene; $c^{\prime}$ corpo cavernoso dell' uretra; $B c, \mathrm{~m}$. bulbo-cavernoso; I c I c, m. ischio-cavernoso.

" 48 - Figura schematica rappresentante il decorso d'un tubulino seminifero.

" 49 - Sezione trasversale della parete del deferente; $e$ epitelio; $b$ basamentale; $l$ fibre muscolari longitudinali; $c$ fibre muscolari circolari; $l^{\prime}$ strato muscolare longitudinale esterno; $a$ avventizia.

"50-51-52 e 55 - Arterie elicine e loro appendici cieche disposte nelle guaine; 50.52. arterie elicine del pene; 51. arteria elicina dell' uretra; 33 . sezione trasversale d'un'arteria elicina.

" $\$ 4$ - Topografia dei genitali maschili interni del cane secondo C. Eckhardt $-v$ vescica urinaria ruota; $r$ intestino retto; $p$ prostata; / funicello spermatico; $d$ deferenti; $v s$ vasi sanguigni del lesticolo; $u$ uretere; $g$ ganglio mesenterico posteriore; $r c$ rami di comunicazione dello stesso col plesso ipogastrico: $\varepsilon$ nervi erettori e loro diramazioni. 
T'av. T.

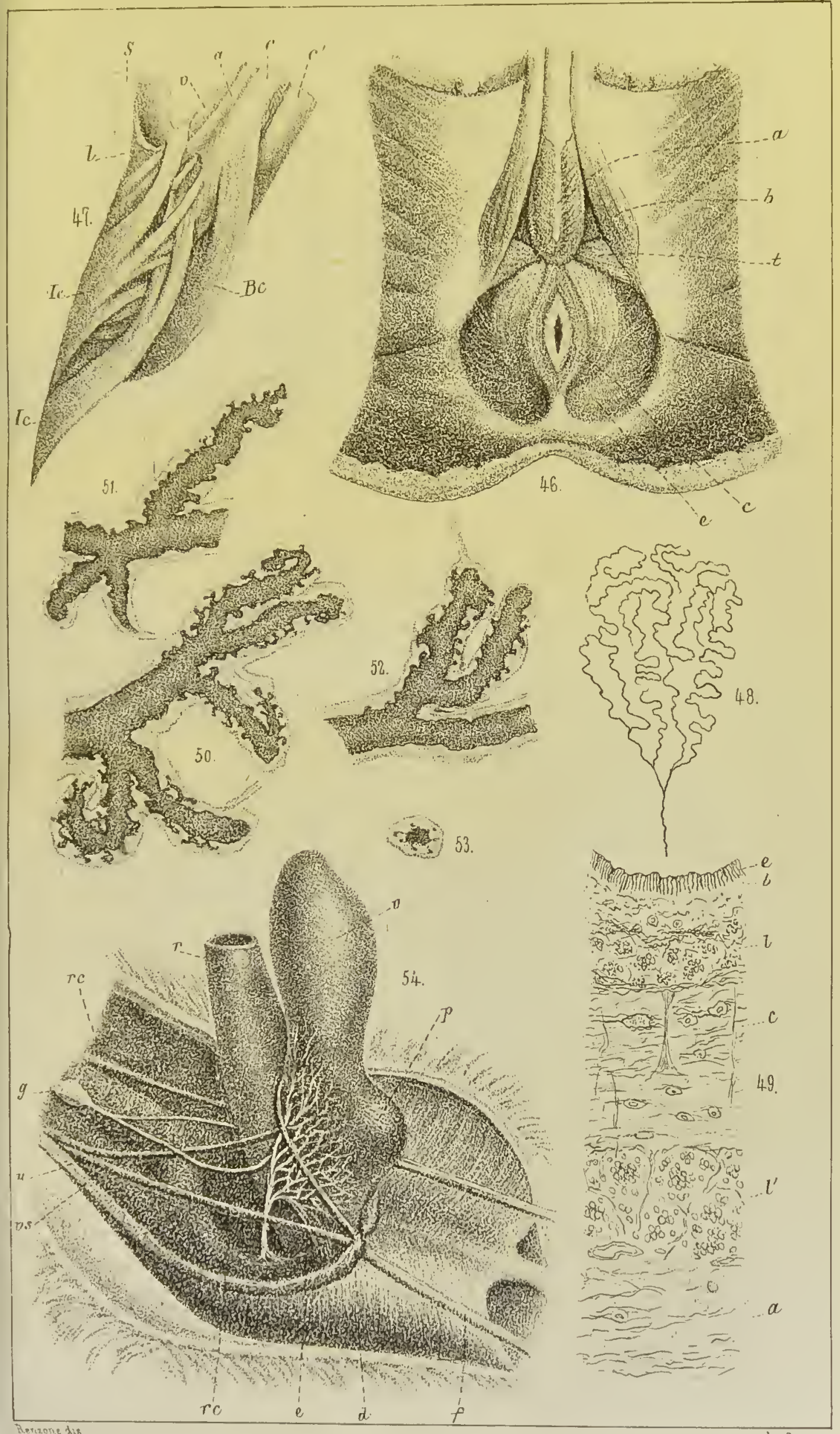



TA V O L A S E S T A 


\section{TH VIA SESTA}

\section{ORGANI GENITALI FEMMINILI}

Fig. 55 - Apparecchio genitale interno della donna (canale utero-vaginale spaccato). $U$ utero; $V$ vagina; $m$ muso di tinea; $c$ u collo dell'utero; $T$ Tuba aperta; $d$ morsus diabuli; $l$ legamento rotondo; $O$ ovaja; $p$ parovario con idatide $i ; v$ o vasi ovarici ; leg legamento largo.

) 56 - Taglio antero-posteriore dell'utero e della porzione superiore della vagina; $p$ pareti; $a$ cavilà; $a v$ alto fondo della vagina alla parte posteriore.

D 57 - Glandula uterina d'una primipara, otto giorni dopo la concezione.

" 58 - Fibro-cellule muscolari d'utero al terzo mese dopo il parto; $c$ cellule trattate con acido acetico; $a$ nucleo; g g granulazioni grasse.

1) 59 - Sezione orizzontale della mucosa uterina; $y g$ sezioni delle glandule con epitelio; $l$ sezione d'una glandula senza epitelio; $v$ vaso sanguigno.

1) 60 - Ovaja d'una donna d'anni 31 che si appiccò durante la mestruazione; $c$ coagulo sanguigno; $d$ pareti del corpo luteo.

" 61 - Ovaja d'una giovenca (spaccato Iongitudinale;) / follicolo quasi maturo; 7 grosso corpo Iuteo aperto; cavita dello stesso ; d piccolo corpo luteo piu vecchio; $e$ altri corpi Iutei piì antichi; $i$ follicoli giovani spaccati; $h$ follicoli giovani chiusi; $v$ rene dell'ovaja; s stroma dell'ovaja con molti vasi tagliali. 



\section{T A V O L A S E T T I II A}




\section{SVILUPPO DEGLI OVULI NELL'OVAJA}

Fig. 62 - Sezione verticale dell'ovaja d'una cagna adulta; $e$ e epitelio peritoneale; $o$ ovulo contenuto nel follicolo $\int$ quasi maturo; $o^{\prime} o^{\prime}$ follicoli più giovani coi loro ovuli; $\iota$ « follicoli ancora più giovani, che per compressione degli altri più sviluppati restano nell'interno dello stroma conservando la traccia della primitiva introflessione peritoneale a forma d'un collo di fiala; $L$ corpo luteo profondo, spinto mol to in dentro per lo sviluppo del follicolo $f ; l$ dotto allungato ed obliterato dello stesso corpo luteo; $c$ c $c$ cisti inveterate o residui di corpi lutei.

" 63 - Sezione normale d'un'ovaja di bambina al sesto mese di vita intrauterina; $s$ s stroma dell'ovaja con vasi e connettivo; c c c c sostanza corticale della stessa, da cui mediante un pennello si è allontanato tutto l'epitelio, restaudo cosi a nudo l'orditura di connetti. vo, la quale presenta tante introflessioni a saccoccia dell'involucro peritoneale. L'epitelio ill esse conteut to era costituito da grosse cellule sferiche e nucleate. Gli otricelli diventano follicoli di Graaf e le cellule contenute costituiscono l'ovulo e la membrana granulosa.

D 64 - Taglio tangenziale dell'ovaja (della stessa bambina) senza epitelio e che presenta le aperture degli otricoli o o o che nello sviluppo si chiudono formando lo stigma.

" 65 - Schema dello sviluppo delle uova nella gallina.

"L'ovaja della gallina sembra costruila secondo il lipo delle glandule a grappolo, ma l'esame accurato della strullura di quesla glandula nédiversi periodi di suo sviluppo c'induce a ritenerla formata da uno stroma 
Fig. - - membranaceo disposto a pliche (vedi Fig. 56. Tavoia (erza) ed ogni plica in sezione normale presenta presso a poco l'aspello della sezione dell'ovaia di bambina alla fig. 65. Siccome nell' ovaja. di gallina i follicoli debbono acquistare uno sviluppo enorme per conlenere il grosso tuorlo di nutrizione, cosi è che essi ben presto si fanno sporgenti fino al punto di diventare peduncolali.

Peritoneo $p, p, p ; v, v, v$, vasi sanguigni dello stroma dell' ovaja; $f$ follicolo piccolissimo ma già sporgente un poco dalla superficie dell'ovaja e contenuto nell'introflessione peritoneale $i$, intorno alla quale si ramificano i vasi dello stroma, senza raggiungersi nel punto d'introflessione, il quale resta occluso da un tessuto di saldamento bianco $c$ che costituisce lo stigma; $f^{\prime}$ follicolo peduncolato, le pareti del quale constano naturalmente di due foglietti, peritoneale esterno $e$, e peritoneale introflesso o proligero $p r(1)$ saldati nello stigma $s$ e divisi fra loro dalla rete vascolare $r r$; follicolo prossimo ad aprirsi $f^{\prime \prime}$; calice o follicolo aperto nello stigma e vuoto $f^{\prime \prime \prime}$.

" 66 - Follicolo maturo di gallina tagliato al peduncolo e che presenta il foglietto esterno e e sollevato dall'interno $p r$ fino ai margini d'introflessione del primo nel secondo, che sono saldati fraloromediante il tessuto bianco che chiude lo stigma, il quale è disposto a semicerchio nel senso del meridiano come un nastrino bianco con estremità ottuse. La figura lineare e non circolare dello stigma $s s$ sembra l'effetto della resistenza dei grossi vasi, che usciti dal peduncolo si portano ai due lati opposti dell'uovo in corrispon-

(1) Questo fogrlietto forma un saces perfettamente chiuso ed unito all'esterno pei soli vasi. 


\section{XVIII}

Hig. - - denza degli estremi dello stigma, e fauno le funzioni di briglie che lo tengono teso.

" 67 - 68 - 69-70-Sviluppo delle uova secondo Pflueger. 67. Tubulino ooforo giovane isolato in cui si distingue il canale, lo scompartimento germinale e la sezione trasversale; $68,69,70$. Calene di ovicini d'una giovane cagna. 


\section{(t)

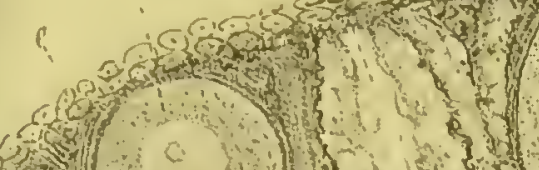 $(-9) 2^{34}$

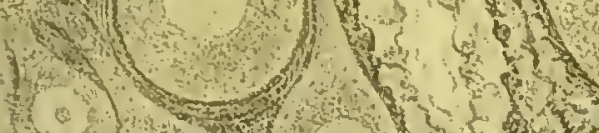 $8 \times$.}

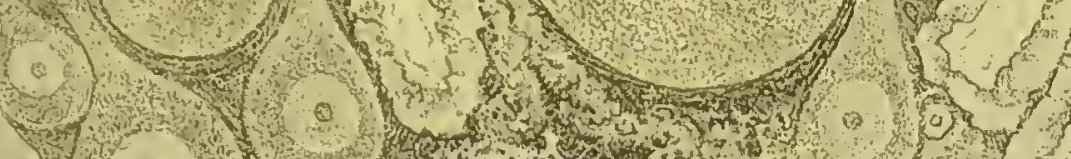

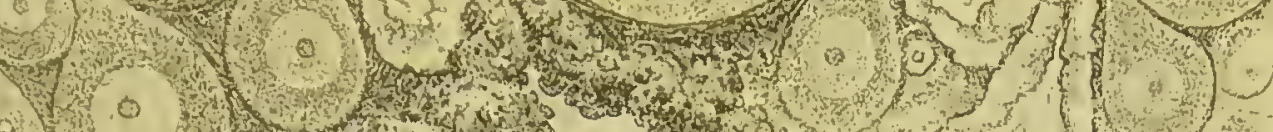

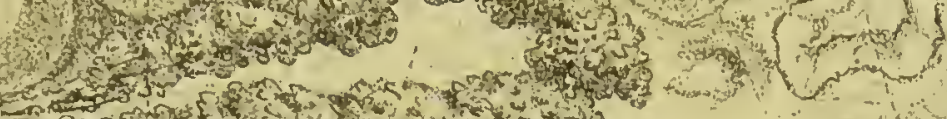
D or

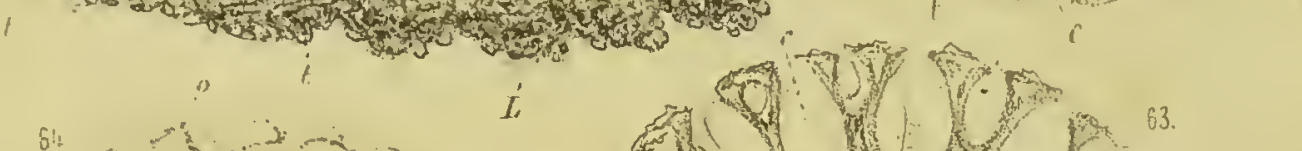

$5+3$

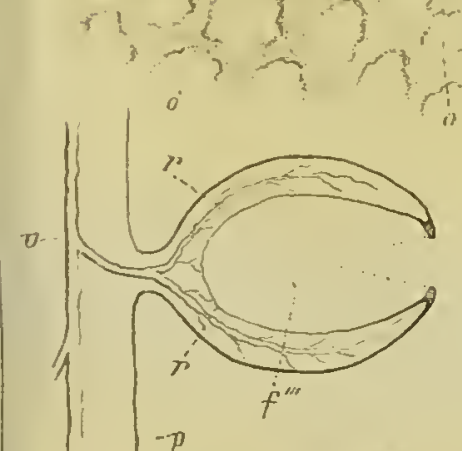
(1) D. (1)

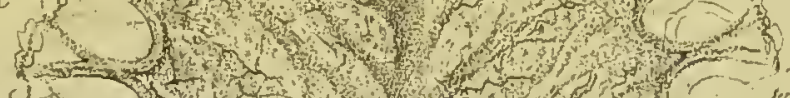

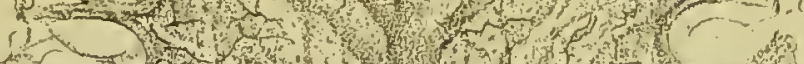

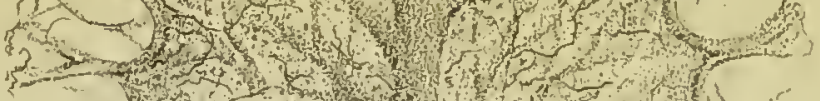

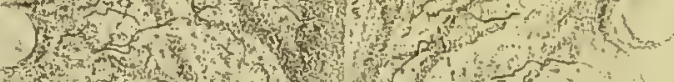

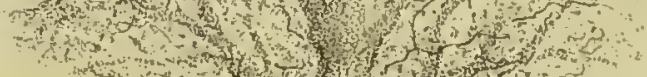

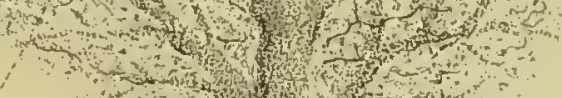
$s \quad-\quad y=$

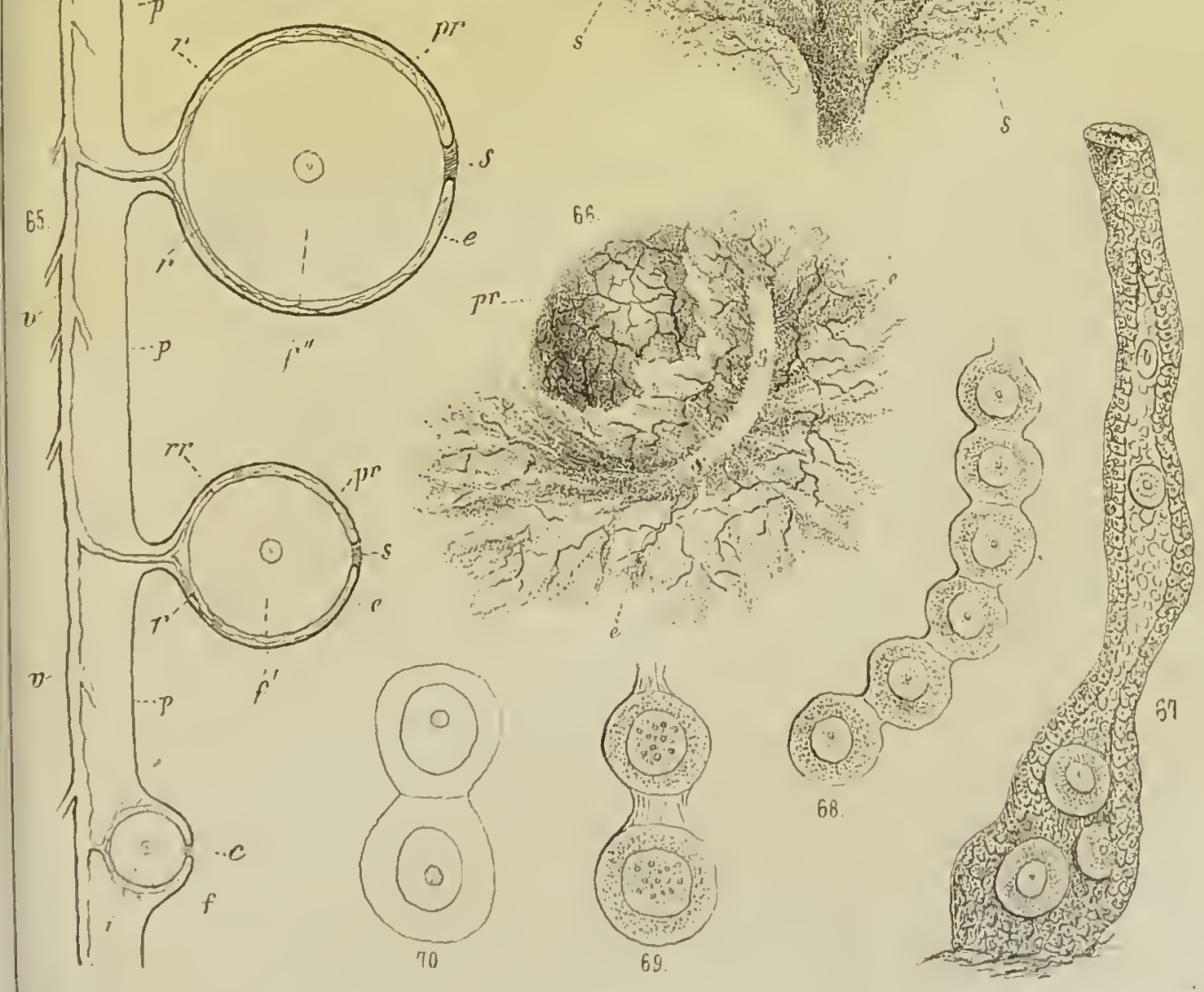



TAVOLA OTTA. A 


\section{OVULI E FLLAMENTI SPERMATICI}

Fig. 71 - Orulo di cane pulito dalle cellule del disco ooforo e rotto in un punto; $z$ zona pellucida; $t$ tuorlo; $v$ vescicola germinativa.

1) 72 - Uovo di una Venus decussata; a micropile.

D75 - Uovo dell'Oloturia tubulosa; a micropile.

"74 - Ovulo di mammifero circondato dalle cellule del disco ooforo; le cellule adossate alla zona pellucida sono allungate e disposte a modo di raggi.

"75 - Cristalli di luleo-ematoidina secondo Piccolo e Lieben, ottenuti dall'estratto etereo dei corpi lutei atrofizzati della giovenca.

1) 76 - Cristalli di ematoidina dal corpo luteo secondo Funke.

1) 77 - Ovulo di Thetis fimbria con filamenti spermatici aderenti $(1)$.

1) 78 e 79 - Filamenti spermatici di diversi animali; $a$ Picus viridis; $b$ Lanius ruficeps; c Fringilla carduelis (ibrido); d Turdus merula; e Cobitis fossilis; f Lepus cuniculus; $g$ Cervus capreolus; $h$ Felis Calus; $i$ Canis familiaris; $k$ Talpa europea; l Hypudaeus arvalis.

b) 80 - Filamenti spermatici dell'uomo.

) 81 - Fascetto di filamenti spermatici dell'uomo tolti dal testicolo.

" 82 - Sviluppo dei filamenti spermatici del cane famigliare secondo Ecker; a $b$ c cellule contenenti vescicole trasparenti; $d$ filamenti spermatici sviluppati nell'interno delle vescicole; $e$ cellula scoppiata per dar uscita al filamento.

(1) Questa fiogura mi venne grentilmente favorita dal Dott. Leone De Sanctis, il quale mi fece osservare che i filamenti spermatiei di questi animali maneauo dell'ingrossamento o testa che vien spesso inentita dal ripiegarsi a eappio del filamento all'uno oppure ad ambedue gli estremi. 


\section{XXI}

Fig. 85 - Sviluppo dei filamenti spermatici nell'uumo (secondo La Vallette); $a$ cellula spermatica con nuclei modificati e filamenti sporgenti; $b$ filamenti spermatici con residui di cellule, $c$ filamenti spermatici apparentemente maturi.

" 84 - Sviluppo dei filamenti spermatici nel cane (secondo La Vallette), $a b c$ cellule spermatiche con nuclei modificati e filamenti sporgenti; $d$ quattro corpuscoli spermatici ancora riuniti dalla sostauza cellulare; $e$ f $g h i$ gradazioni di sviluppo degli stessi corpuscoli. 



\section{Tav TIII.}
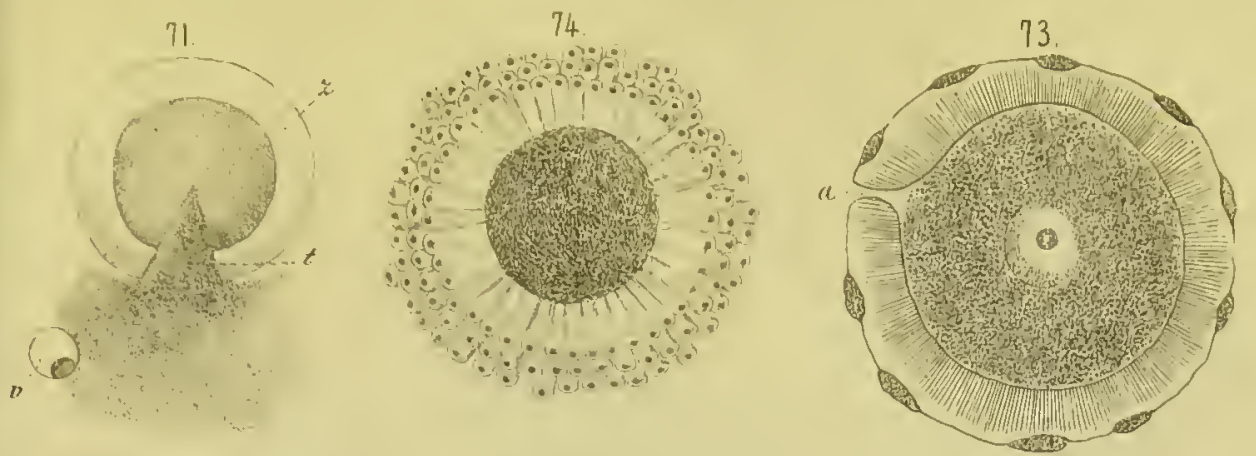

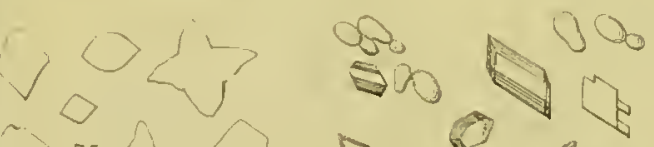

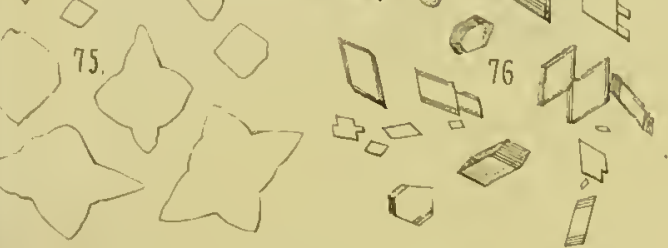
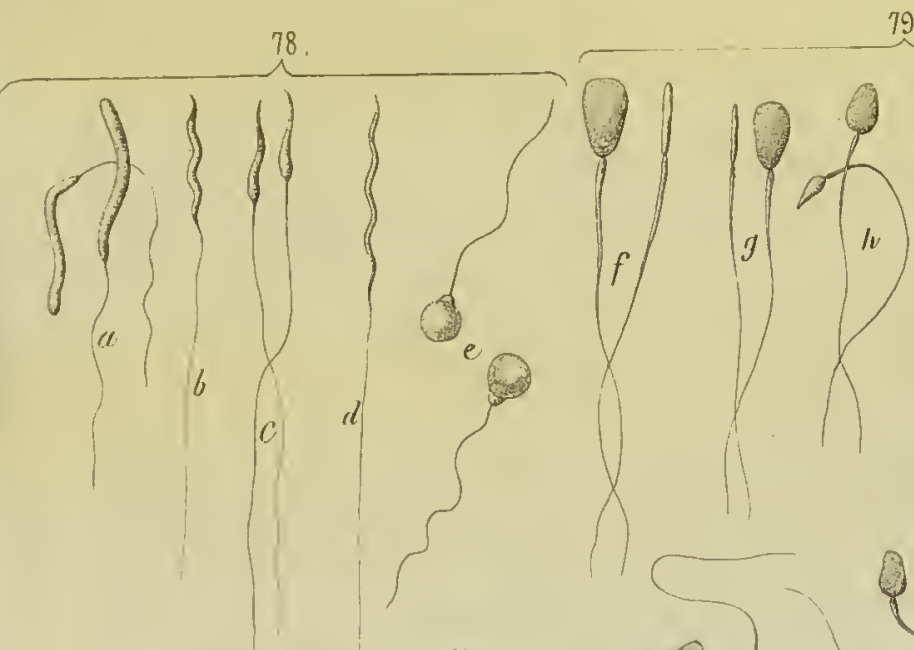

79.
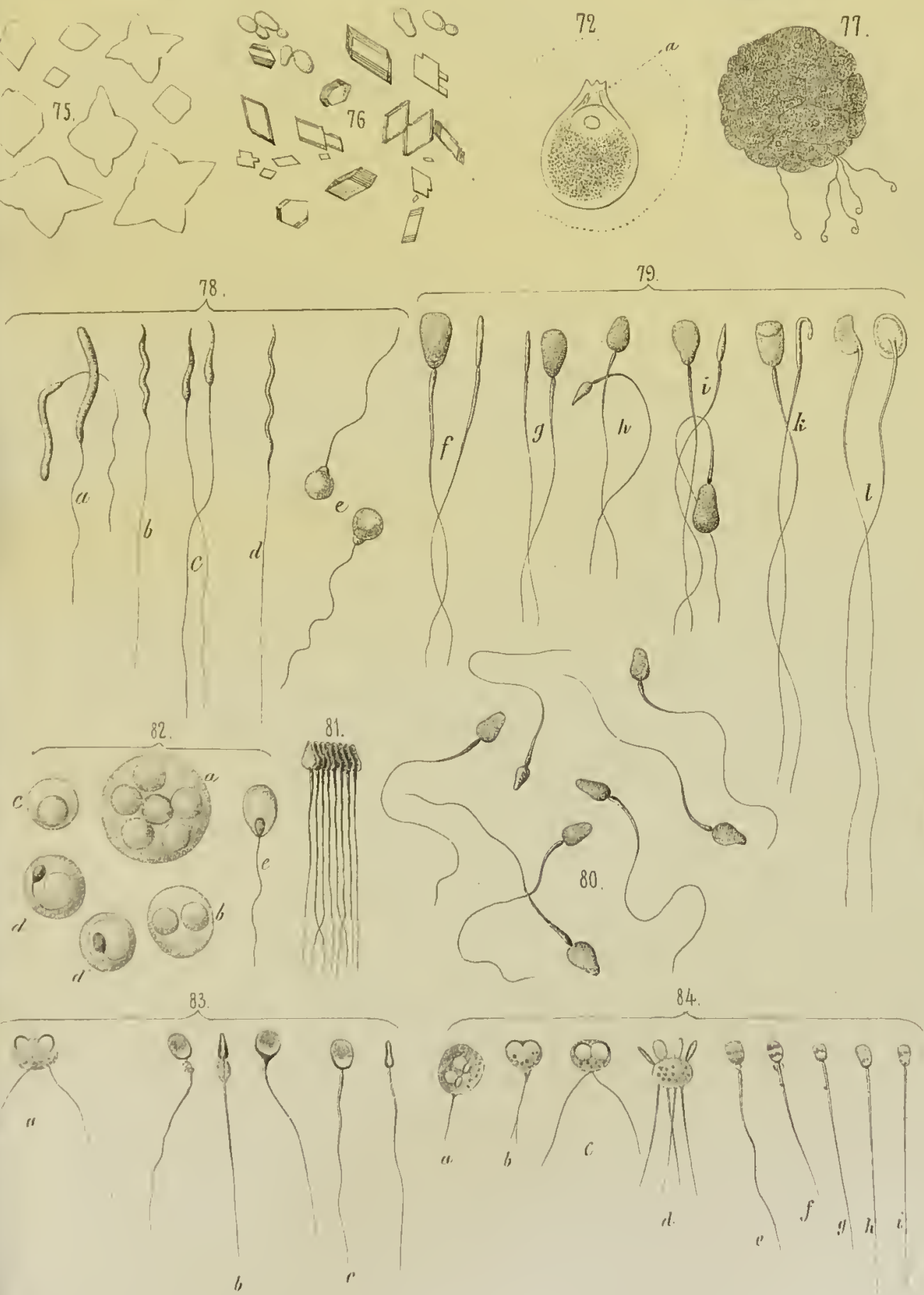

TA V O L A N O N A 


\section{TAVOIAA NONA}

PRIMI CAMBIAMENTI DELI'UOVO DOPO L'INCONTRO COI SEMF.

Fig. 85 - Uovo di coniglia proveniente dal mezzo della tromba circondato da uno strato d'albume $s$ con filamenti spermatici; $\iota$ tuorlo; $m$ masse polari

1) 86 - Idem. - Inizio della segmentazione. Il tuorlo é diviso in due metà ovali, in ognuna delle quali si osserva una macchia chiara.

b 87 - Idem, di quattro ore più tardi ; il tuorlo è diviso in quattro masse sferiche.

" 88 - Idem, al terzo inferiore della tromba.Lo strato d'albumina è molto spesso. Il tuorlo è diviso in molte sfere.

1) 89 - Idem, dal fine della tromba; lo strato dell'albume è ancora più grosso e le masse ancora più numerose ; i filamenti spermatici molto scarsi.

" 90 - Idem, un poco più abbasso nell'utero; vi si scoprono le masse di segmentazione ridotte a cellule poligonali; $z$ zona pellucida assottigliata; $a$ a mmasso di sfere vitelline; filamenti spermatici ancora più scarsi.

" 91 - Idem, uovo uterino ingrossato; zona pellucida $\approx$ ed albume assottigliati; $i$ cellule poligonali e nucleate formanti il blostoderma; $a$ ammasso di sfere vitelline, non ancora impiegate nella formazione del blastoderma.

" 92 - Uovo uterino di sette od otto giorni veduto di profilo; $p$ zona pellucida coi principii delle villosità; Ia membrana interna $b$, blastoderma, presenta nel segmento superiore l' area germinativa $g$ di apparenza più bianca.

" 95 - Figura semiscliematica dell' embrione umano nei suoi primordii ; $c$ corpo dell' embrione in cui già si distingue l' estremità cefalica più voluminosa: l'amnios $a$; la vescicola umbelicale $v$; la zona pellucida $z ;$ c le villosità del chorion $c h$. 

TA VOLA DEGINA 
OIUGINE DEL. CORPO DELL'ANIMALE VERTEBRA'TO DALL' AREA GERMINATIVA

Fig. 94-Membrana germinativa dell'uovo di gallina in principio dell'incubazione (veduta dal di sotto ed ingrandita circa venti volte) secondo Remak; ok foglietto germinativo superiore; do margine del foglietto germinativo inferiore con grosse cellule oscure che rappresentano l'area opaca; $d s$ inspessiniento centrale dell' area pellucida formato dal foglietto mediano e superiore; ap nota primitiva dove i due foglietti sono saldati tra loro.

1) 95 - Sezione normale della stessa area. Per maggior chiarezza si è lascialo uno spazio fra i foglielli; ap, $o k$, do, come nella figura precedente; $m k$ foglietto mediano; $d$ parte mediana più sottile del foglietto interno o foglietto mucoso-iutestinale; ed parti confinanti col tuorlo.

) 96 - Area pellucida ovale di un uovo di gallina (ingrandita venti volte e vedutadalla parte addominale) secondo Remak; ch corda dorsale ; $x$ rigonfiamento alla parte posteriore della stessa; ap metà laterali delle piastrine marginali della doccia primitiva, che all'estremità cefalica z passalı ad arco l'una nell' altra ed all'estremità caudale si assottigliano passando nel blastoderma; $S p$ piastrine laterali che passano l'uıa nell'altra tanto all'estremità caudale come all'estremità cefalica; $y$ limiti dell'area pellucida.

1) 97 - Sezione normale a metà della stessa area ; ch corda dorsale; $\mathrm{mm}$ vertebre primitive allo stato di piastrine che si continuano nelle laterali ; $m p$ piastrina midollare, che si continua nel foglietto corneo-epidermoidale; $d$ doccia primitiva; $s$ foglietto mucoso. 
Fig. 98 - Sezione schematica del corpo umano; $m$ canale del midollo spiuale compreso nello speco vertebrale; $v$ vertebra; $c$ coste ; ci tubo intestinale formato dalla mucosa $m c$, dalla muscolare $m u$, e dal peritoneo viscerale $p v$; mes mesenterio ; $p p$ periloneo parietalc; $g v$ vasi sanguigni maggiori; mi muscoli della parete del tronco; $t g$ tegumenti comuni.

» 99 - Sezione normale del tronco di un embrione di pollo al principio del quinto giorno (ingrandimento 90-100 diam.); $\mathrm{cm}$ canale midollare; sh guaina della corda dorsale; $h$ foglietto corneo-epidermoidale; $a m$ amnios quasi cliuso nella linea mediana dorsalc in $y$; $s a$ aorta secondaria; $v c$ vene cardinali; $m u$ lamina musculare della pagina cutanea del foglietto mediano;g ganglio spinale; $v$ radice anteriore d'un ncrvo spinale; $h p$ lamina cutanea della pagina esterna del foglietto mediano; ap continuazione delle vcrtebre primitive nelle pareti addominali ; $b h$ parete addominale primitiva formata dalla lamina cutanea e dal foglietto corneo-epidermoidale; df parete fibrosa del tubo intestinale formata dalla pagina interna del foglietto mediano; $d$ foglietto epiteliale della mucosa intestinale. Le pareti dell'intestino si vedono inspessite posteriormente cd assottigliate verso la linea mediana anteriore, dove sono in contilluazione mediante il dotto omfalo-meseraico om colla vescicola ombelicale vo. La massa che circonda la corda dorsale è il corpo delle vertebrc in via di formazione; la massa che trovasi al davanti dei vasi sanguigni contiene dei rigonfiamenti laterali che sono i corpi del Wolff, e nella linea mediana si continua nel mesenterio.

"100 - Sezione normale del corpo d'un embrione di pollo alla fine del primo giorno d'incubazione (ingrandimento 15 diam.) secondo Remak; ch corda dorsale; 
$d$ foglictto epitelialc-mucoso; $u$ vertebre primitive; $s p$ piastrine laterali; $m$ piastrina midollare ; $i$ hordi sporgenti della doccia midollare; $h$ fogliello corneo-epidermoidale.

Fig. 101-Sezione normale del primo rudimento del corpó di un embrione di pulcino alla fine del primo giorno d'incubazione (ingrandimento $90-100$ diametri) ch. corda dorsale; uoh vertebre primitive con fessura o cavità centrale uvh; $s p$ piastrine Iaterali in continuazione delle vertebre primilive; $d d$ foglietto mucoso-epiteliale ; $h$ foglietto corneo-epidermoidale ; $m$ piastrina midollare. Ambeduc le piastrine midollari si vedono sollevate a formare o limitare la doccia midollare $\boldsymbol{R} /$, nella cui linea mediana si vede ancora la doccia primitiva $R$.

) 102 - Sezione del corpo d'un embrione di pollo alla fine del primo giorno, un poco indietro dell'apertura ilell'intestino primitivo anteriore. La doccia midollare è largamente aperta, lo stesso vale per l'intestino primitivo; le pagine laterali del foglictto mediano sono divise nella pagina parietale del tronco e nella pagina parietale intestinale ; ai margini dell'embrione si vedono innalzarsi tre foglietti dell'area germinativa per formare due ripiegature laterali che sono il principio dell' amnios ; $s p$ cavità pleuro-peritoneale.

1) 105-104-Embrione di pollo al principio del secondo giorno d' incubazione (Fig. 103 veduto dal dorso; Fig. 104. veduto dall' addome); $a$ corpo dell' embrione: $b$ rigonfiamento cerebrale; $c$ commissura coccigea dei margini delle lamine parietali ; $d d$ masse subprismatiche ; ee corda dorsale : / branca superiore : g!? branca inferiore del cappuccio cefalico.

Anuotazione-Dalle figure $99,100,101,102$, si rileva evidentemente la trasformazione graduata della figuril 95 nella figura 98. 
Tav.X.

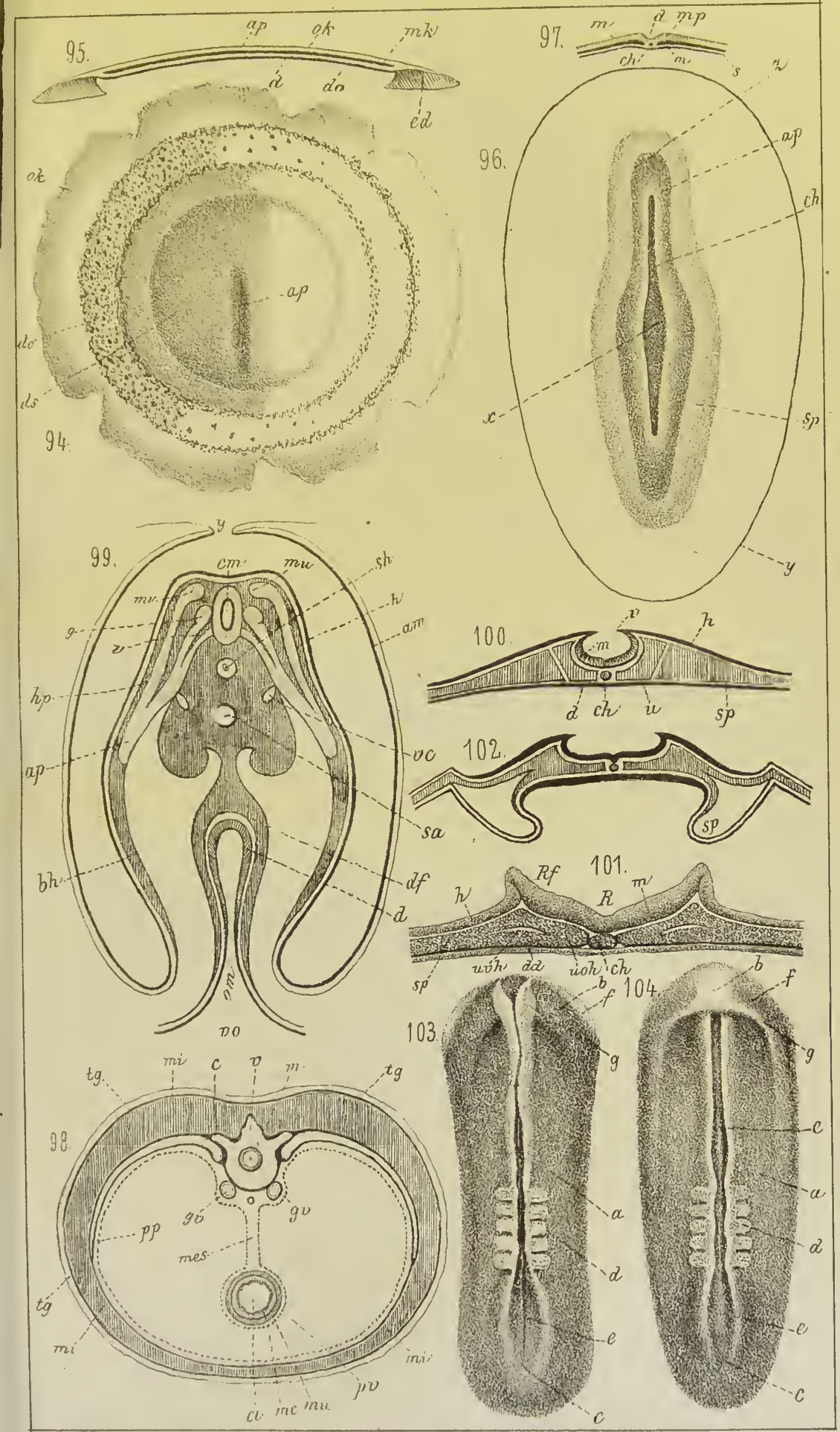



T A VOLA U N DEC I II A 


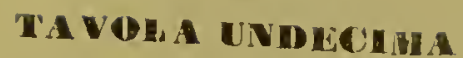

SVILUPPO DEGL' INVOLUCRI ED ORGANI APPEVDICOLARI DELL' EMBRIONE

Fig. 105- Spaccato dell'uovo in cui si vede la zona pellucida, il blastoderma e l'area germinativa; la parte centrale più grossa rappresenta lo spaccato trasversale del rudimento dell'embrione.

" 106 - Spaccato d'un uovo in direzione della lunghezza dell'embrione. Inizio dell'amnios e della vescicola ombelicale.

" 107 - Spaccato d'un uovo come nella fig. antecedente. II sacco dell' amnios si chiude e compare l'allantoide come un bottone piriforme.

1) 108 - Spaccato come sopra. L' uovo è circonda to dal secondo chorion villoso; allantoide assai sviluppata.

1) 109 - Uovo in cui lo strato vascolare dell' allantoide si è già adattato alla faccia interna del chorion sieroso o secondo, ed i suoi vasi sono già penetrati nei villi. La vescicola ombelicale è ridotta a minime proporzioni, il sacco dell'amnios assai ingrandito.

Spiegazione delle lettere comunia queste cinque figure. $d$ membrana vitellina o zona pellucida; $d^{\prime}$ piccoli villi della membrana vitellina; $s z$ villi della membrana sierosa; ch chorion villoso vascolare formato dallo strato vascolare dell' allantoide; chz veri villi del chorion, costituiti dai processi del chorion vascolare rivestiti dalla membrana sierosa ; am amnios; ks guaina caudale dell'amnios; ss guaina cefalica dell' amnios; ah cavità del sacco dell' amnios; as guaina dell'amnios pel cordone ombelicale; $a$ inspessimento del foglietto esterno del blastoderma $a^{\prime}$ in corrispondenza del rudimento del corpo dell'embrione; $m$ inspessimento del fogliello mediano 
dell'area germinativa $m^{\prime}$, che in principio non oltrepassa il limite dell' area, e più tardi rappresenta lo strato vascolare della vescicola ombelicale $d s ; s \iota$ seno terminale; $d d$ strato mucoso glandulare del tubo digerente formatosi dal foglietto interno del blastoderma in corrispondenza dell'area; $k h$ cavità delIa vescicola blastodermica che in seguito diventa vescicola ombelicale $d s$; $d g$ dotto omfalo-mesenterico ; al allantoide; $e$ embrione; $r$ spazio primitivo fra l'amnios ed il chorion ripieno di liquido albuminoso; $v l$ parete anteriore del corpo nella regione del cuore; hh posto occupato dal cuore. Per maggior chiarezza nella Fig. 106 l'amnios è disegnato alquanto discosto dal corpo dell'embrione; per la stessa ragione il sacco del pericardio è lenuto più piccolo.

Fig.110-Sezione schematica dell'uovo umano all'epoca dello sviluppo dell' allantoide; $d$ membrana vitellina; $a b$ foglietto sieroso o corneo-epidermoidale del blastoderma; $v b$ foglietto vegetativo o mucoso che si continua nella vescicola ombelicale; am amnios che al segno * passa nel foglio corneo-epidermoidale; al allanioide; $n$ vescicola ombelicale.

" 111 - Embrione umano forse non affalto normale. L'amnios am si continua all' ombelico immediatamente nelle pareti addominali: Per l'anello ombelicale si vede uscire anteriormente il dotto omfalomeseraico che va alla vescicola ombelicale $n$; posteriormente il funicello ombelicale $\int$ che è ancora allo stato di doccia. Dietro del processo mascellare superiore ok si vedono il $1^{0}$ e $2^{\circ}$ arco branchiale ed in o si osserva la vescicola uditiva.

" $1 / 2$ - Embrione umano Inugo circa $4{ }^{1} / 2$ "', in cui si vedono assai chiaramente due archi branchiali e le singole vescicole cerebrali; l'amnios è tolto e la ve- 
scicola ombelicale $n$ è stata aperta; $c$ chorion; $v v$ villosità dello stesso.

Fig. 115-Sezione scliematica dell'utero gravido contenente l'embrione; l trombe faloppianc; $c$ collo dell'utero; du decidua vera; $d r$ decidua riflessa; $d s$ decidua scrotina; ch chorion; am amnios chc passa nella guaina del funicello ombelicale; al allantoide, i cui vasi s'innoltrano nei villi per formare la placenta fetale. I villi nclle altre parti dell'uovo mallcano di vasied una volta che si è sviluppata la placenta servono soltanto a tener fisso l'ovicino ; $a b$ vescicola ombelicale che per mezzo del suo picciuolo o dolto omfalo-meseraico si continua nellc pareti dell'intestino $i$.

) 144-Placenta umana. La figura rappresenta una sczione verlicale schematica dell'utero, della placenta e del chorion nella donna; $a$ chorion aderente alla superficie fetale della placenta; $b$ vasi arteriosi e venosi in rapporto con quelli del funicello ombelicale, dai quali nascono le villosità costituenti la porzione fetale della placenta; $c c$ villositá, le quali si reggono completamente avolte da una guaina ddd lal cliorion all'utero; la guaina ha origiıe dalla decidua serotina fl ed è formata da una membrana anista esterna c da uno strato epileliale interno ; ec parete dellutero; $h$ un villo della placenta felale a cui ì stato tolto l'invoglio esterno (1).

(1) L'Ercolani da cui abbiamo presa questa figura considera lo strato epiteliale interno della guaina come un orano glandulare. 


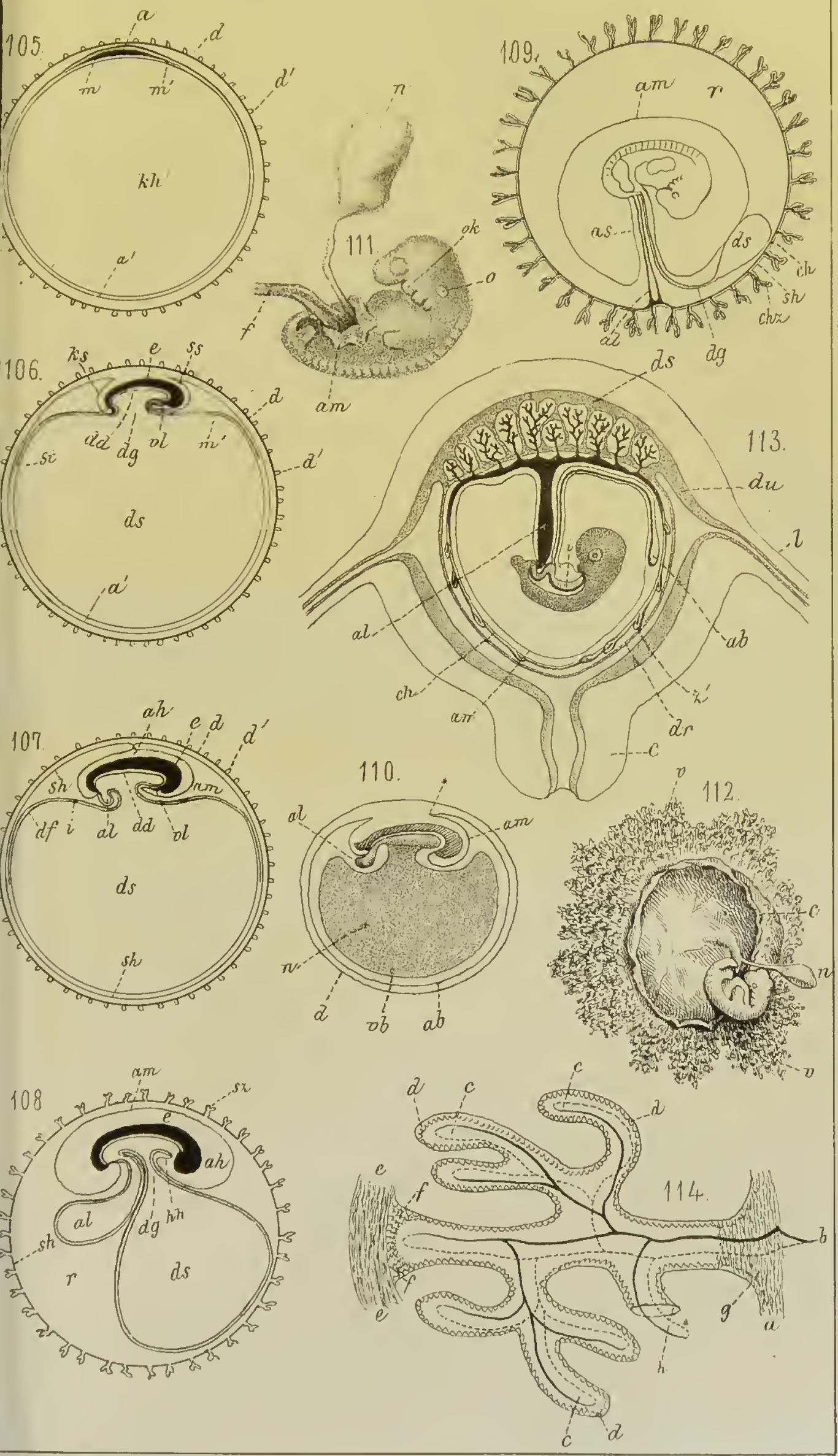



T A V O L A D U O D E C I II A 


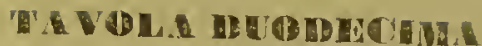

SVILUPPO DELLA PLACENTA NELIE SUE HIVERSE FORME

Fig. 115 - Schema dell'uovo umano nel secondo mese di gestazione; $a$ porzione liscia del chorion; a' porzione villosa dello stesso; $k k$ villi allungati clıe cominciano a raggrupparsi per formare la placenta ; $b$ vescicola ombelicale; $e$ embrione; am amnios; al allantoide.

" 116 - Alcuni ramoscelli vascolari di un villo del chorion iniettati; $a$ tronco vascolare principale; $n$ capillari della rete superficiale.

" 117 - Primo stadio di formazione della decidua riflessaintorno all'ovulo; $o$ ovulo con villi; $d r$ decidua rillessa; ds decidua serotina.

1) 118 - Figura schematica per dimostrare come si forma la membrana decidua riflessa; $\iota$ apertura delle tube; $u \iota$ pareti muscolari dell'utero ; $o$ ovulo; $d r$ decidua riflessa; $d v$ decidua vera.

" 119 - Cotiledoni nei rurninanti (disegno tolto da un preparato nello spirito) $u$ pareti dell' utero con calici cc; ch chorion con fiocchetti vascolari; $v$ focchet ti peduncolati e chiusi nei calici.

"120 - Feto di riccio con placenta $p$ sul dorso.

"121 - Feto di cane chiuso nel chorion con placenta zonale $p z$.

"122-Apparato urogenitale nell' embrione del pollo ; aa corpi del Wolff; $b b$ dotti escretori degli stessi; $d u-$ retere; $e$ glandule genitali; $c$ cloaca.

\section{RUDIMENTI DELLO SCHELETRO}

1) 125 - Pezzo della corda dorsale d' un piccolo embrione di pecora; $a$ guaina della corda; $b$ cellule con spazii centrali rescicolari trasparenti. 
Fig. 121-Sezione longiludinale (in corrispondenza della corda) di alcune vertebre toraciclie d'un embrione umano di otto settimane; $v$ corpo delle vertebre cartilaginee; $l i$ legamenti intervertebrali; ch rigonfiamenti iutervertebrali della corda.

" 125 --Sezione trasversale d'una vertebra toracica d'un $\mathrm{cm}$ brione umano dell' età di otto seltimane; ch corda; cv corpo cartilagineo della vertebra; $c$ costa; $a$ nevroapofisi; $p \iota$ processi trosversi.

"126 - Spaccato mediano longitudinale della metà anterio re del corpo d'un embrione di pollo alla fine del primo giorno (figura schematica secondo Remak); $h$ foglietto corneo-epidermoidale; $k$ piastrine o lamine cefaliche; $m$ tubo midollare; $c h$ corda dorsale ; $d$ foglietto mucoso intestinale; df pagina fibrosa del foglietto mediano in corrispondenza dell' intestino anteriore (da questa si forma,il cuorce); $h k$ cavità del cuore; $k k$ cappuccio cardiaco; $k s$ guaina cefalica; $c$ cappuccio cefalico. 



\section{Tav.XII.}

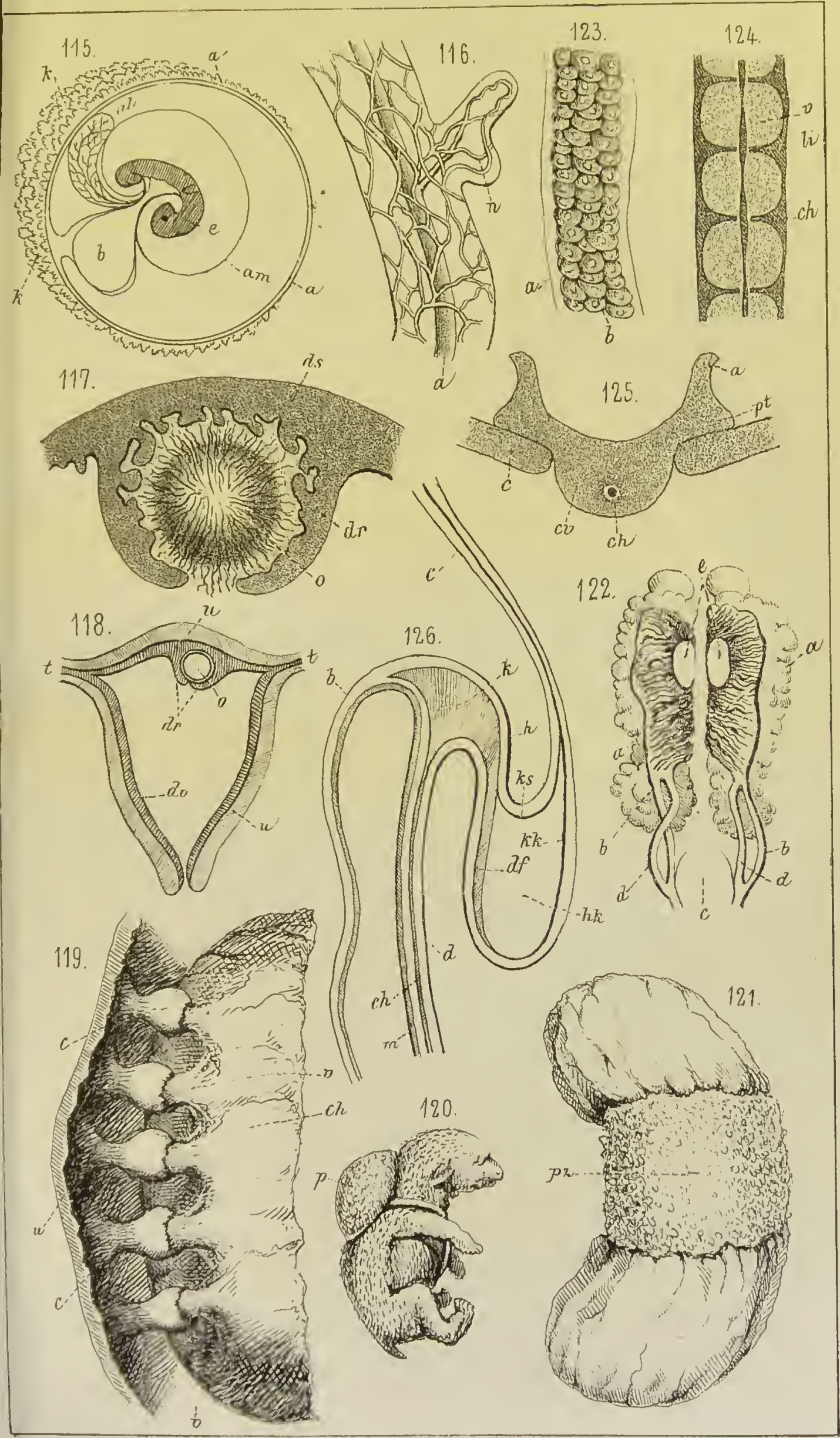



TA VOLA DEG III A TERZA 


\section{TA VOA.A IDECRMATHEZA}

\section{SVILUPPO IEI. SISTEMA NEIIVOSO CENTRALE}

Fig. 127 - Melà d' una sczione normale di un cmbrione di pollo del sccondo giorno d' incubazione (ingrand. 90-100 diam.); ch corda dorsalc; dd foglio mucoso intestinalc; $u v$ vertebre primitive; uvh cavità delle vertebre primitive; ao aortc primitive; ung dotto del corpo del Wolff; $n n$ corpo del Wolff; $m$ lamina muscolare ; $p$ cavità pleuro-peritoncalc; af piega amniotica; $\mathrm{cm}$ canale midollare; $h$ lamina cornca; hb lamina cutanea del foglietto mediano; df parete dell'intestino.

1) 128 - Embrione di cane tolto dalla madre uccísa 24 giorni dopo il coito (ingrandito nove voltc); $a$ blastoderma (foglictto vegetativo); $b$ foglictto motoriogerminativo cle passa dal blastoderma al corpo dell' embrione ; $p$ plica caudale dell' amnios ; $d$ ripiegature contenenti le venc omfalo-mesentcriche; $e$ piastrinc vertebrali; $m$ tubo midollare chiuso; $c p$ epencefalo; $m s$ mesencefalo; $o$ vescicola uditiva. Il prosencefalo non ì visibile perchc̀ piegato innanzi.

1) 129-150-Estremità cefalica del tubo midollare d'un cmbrione di cane di 24 giorni (Fig. 129 dal dorso, Fig. 130 dal davanti); $m$ tubo midollarc chiuso; ep epencefalo assai aperto; ms mesencefalo; $p$ prosencefalo, la cui porzione anteriore incomincia a formare i grandi emisferi $\mathrm{ef}$.

1) 151-152-Embrione umano di circa sei settimane(Fig. 131 dal dorso, 132 dal davanti); $s$ scno romboidale 0 quarto ventricolo; $m s$ corpi quadrigemelli o mcsencefálo; $\mathrm{cr}$ cervelletto ; $m$ midollo spinale; g cstremità caudale Icll'embrionc ; pis prosencefalo coi grandi cmisferi ef. 
Fig. 155 - Lo stesso embrione (visto di lato); c4 grande cmisfero; $c^{2}$ porzione posteriore del prosencefalo; $c^{3}$ mesencefalo; $c^{4}$ cervelletto; $c^{b}$ seno romboidale.

s 15. - Cervello e midollo spinale d'un embrione umano lungo dieci linec (ingrandito); $m$ midollo spinale; $m^{1}$ ginocchio cervicale dello stesso dove passa nel midollo allungato; $c^{5}$ angolo pronuncialo tra la midolla allungata ed il cervelletto $c^{k}$; all' estremilà anteriore di questo ginocchio si sviluppa più tardi il ponte $p ; s$ quarto ventricolo $; c^{3}$ mesencefalo; $c^{2}$ talamo ollico ed olfattorio; l'estremità di questa vescicola rapprescnta l'estremità anteriore del tubo midollare, il quale tanto superiormente come ai lati è già coperto dal grande emisfero $c^{\prime}$; $c a$ corpo calloso.

b 155 - Mesencefalo, prosencefalo e grandi emisferi dello stesso embrione veduti dal davanti; $c^{1}$ grandi emisferi; $c^{2}$ prosencefalo; $c^{3}$ mesencefalo; $p c$ peduncoli del cervello che si vedono al fondo fra il prosencefalo ed il mesencefalo.

3) 156 - Embrione lungo circa $8 \% 2 "$ ( veduto da un lato col cervello e midollo spinale scoperto); $m$ midollo spinale; $c^{5}$ midolla allungata; $g$ ginocchio cervicale del tubo midollare; $c^{4}$ cervelletlo; $s$ seno romboidale; $g^{2}$ altro ginocchio del tubo midollare che diventa il ponte del Varolio $; c^{3}$ eminenze quadrigemellc. In questo punto si osscrva un altro ginocchio del tubo midollare la cui concavilà vienc ad essere occupata dal corpo calloso ; $c^{2}$ talami oltici ; $c^{2}$ prosencefalo coperlo dai grandi emisferi; $f$ Cossa del Silvio.

D 137 - Cervello dello stesso cmbrione veduto di dietru; $m$ midollo spinale; $c^{3}$ midolla allungata ; $s$ quarto ventricolo ; $c^{h}$ cervelletto; $c^{3}$ corpi quadrigemelli. "158-Cervello dello stesso embrione veduto dall'alto; 
$c^{4}$ grandi emisferi; $c^{2}$ talami oflici; $c^{3}$ corpi quadrigemelli.

Fig. 159 - Embrione umano più avanzato del precedente (iugrandito due volte) le lettere come in fig. 136.

" 140 - Lo stesso embrione veduto di dietro; mesencefalo aperto: le lettere come in figura 136.

"141-142-Cervello di un embrione umano lungo 2 pollici, cioè di circa 12 settimane (141 veduto di lato; 142 di dietro); $c^{4}$ cervelletto; $c^{3}$ corpi quadrigemelli non ancora coperti dagli emisferi ; $c^{4}$ emisferi colle circonvoluzioni transitoric; / fossa del Silvio; $c^{5}$ midollo allungato, in cui si vedono i cordoni posteriori, cioè: $m g$ funicello gracile colla clava; $m c$ funicello cuneiforme; $m l$ funicello laterale; $s$ seno romboidale.

n 145 - Cervello dello stesso embrione veduto dall' al to coi grandi emisferi divaricati; $c^{1} ; c^{2} ; c^{3} ; c^{4} ; c^{3}$ come nelle figure precedenti. Sul fondo del grande emisfero sinistro ed aperto si vede il corpo striato cs: $t$ lenia semicircolare; $c c$ corpo calloso; $v$ terzo ventricolo.

1) 144 - Sezione mediana del cervello d' un embrione umano di circa tre mesi; $i$ infundibulo; le altre lettere come nelle figure precedenti. 


\section{Tav. XIII.}

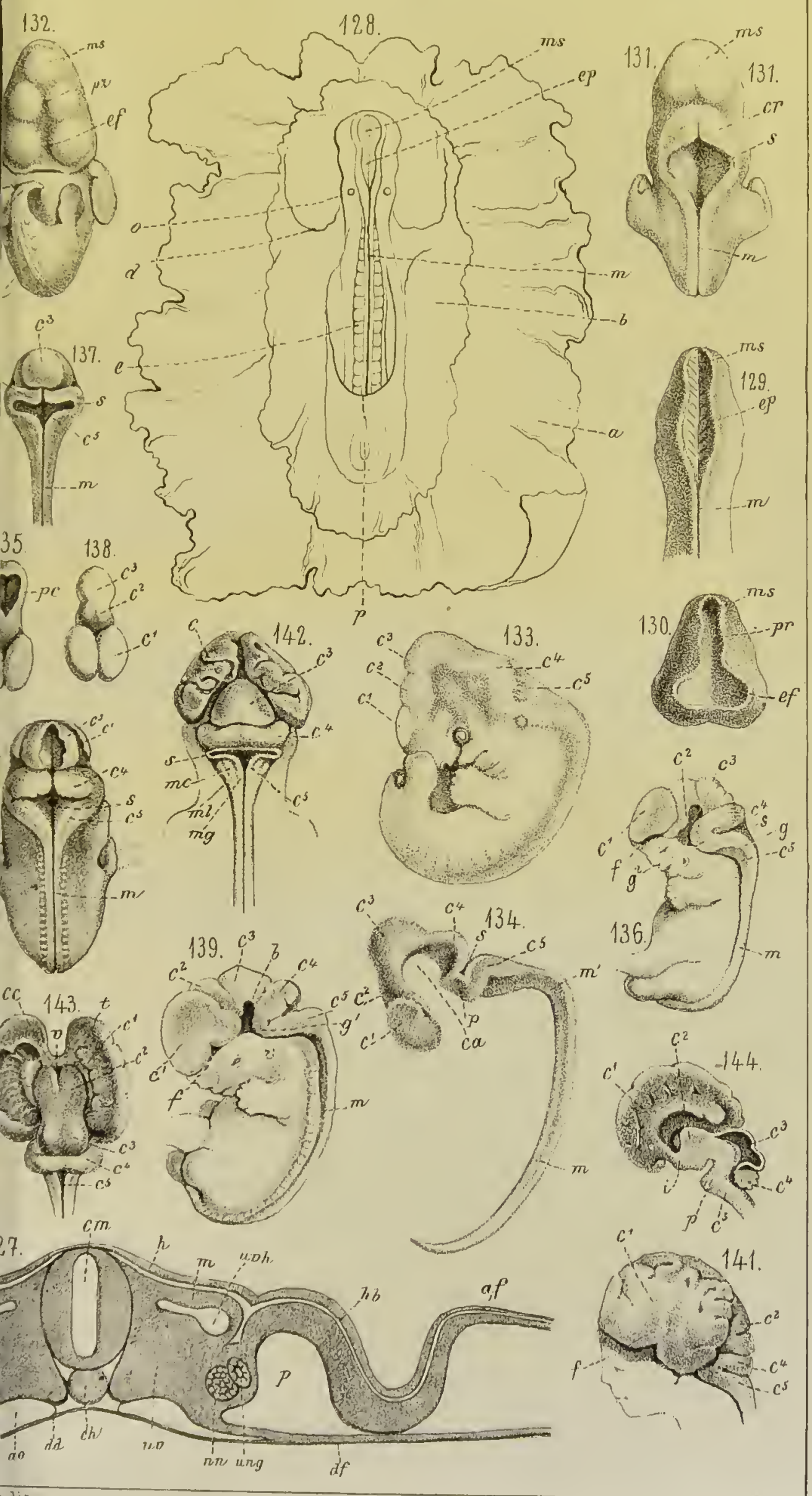





\section{TAVOLA DECIMAOUARTA}


Fig. 156-Metà anteriore dell' osso sfenoide dello stesso animale (veduta dall' alto); $c$ corpo; pa piccole ali o nevro-apolisi; pi processi inferiori o emato-apofisi.

157 - Metà posteriore del corpo dello sfenoide dello stesso animale ; $c$ corpo; ga grandi ali o nevro-apofisi; (1) pi processi inferiori od emato-apofisi.

1) 158 - Osso basilare dello stesso animale veduto dall' alto; $c$ corpo; $p a$ processi condiloidei o nevro-apofisi; pi processi inferiori od emato-apofisi.

Considerando queste tre figure si vede in esse chiaramente il tipo vertebrale.

Il processo anteriore le della figura 156 , che è il corpo dell'osso elmoide, polrebbe essere consideralo come il corpo di un osso di tipo vertebrale, il quale si è saldalo colla vertebra successiva o me là anteriore dello sfenoide.

Le figure 155, 156, 157, 158, furono prese da preparali d' un felo di pecora giunlo quasi a perfezione non nell'ulero ma in un sacco membranaceo nella cavilà addominale per gravidanza extrauterind.

(1) Volendo giudicare dalle dimensioni le granli ali sarebbero quelle della metà anteriore dello sfenoide e le piccole quelle dolla metà posicriore. 
Tav.XIV.

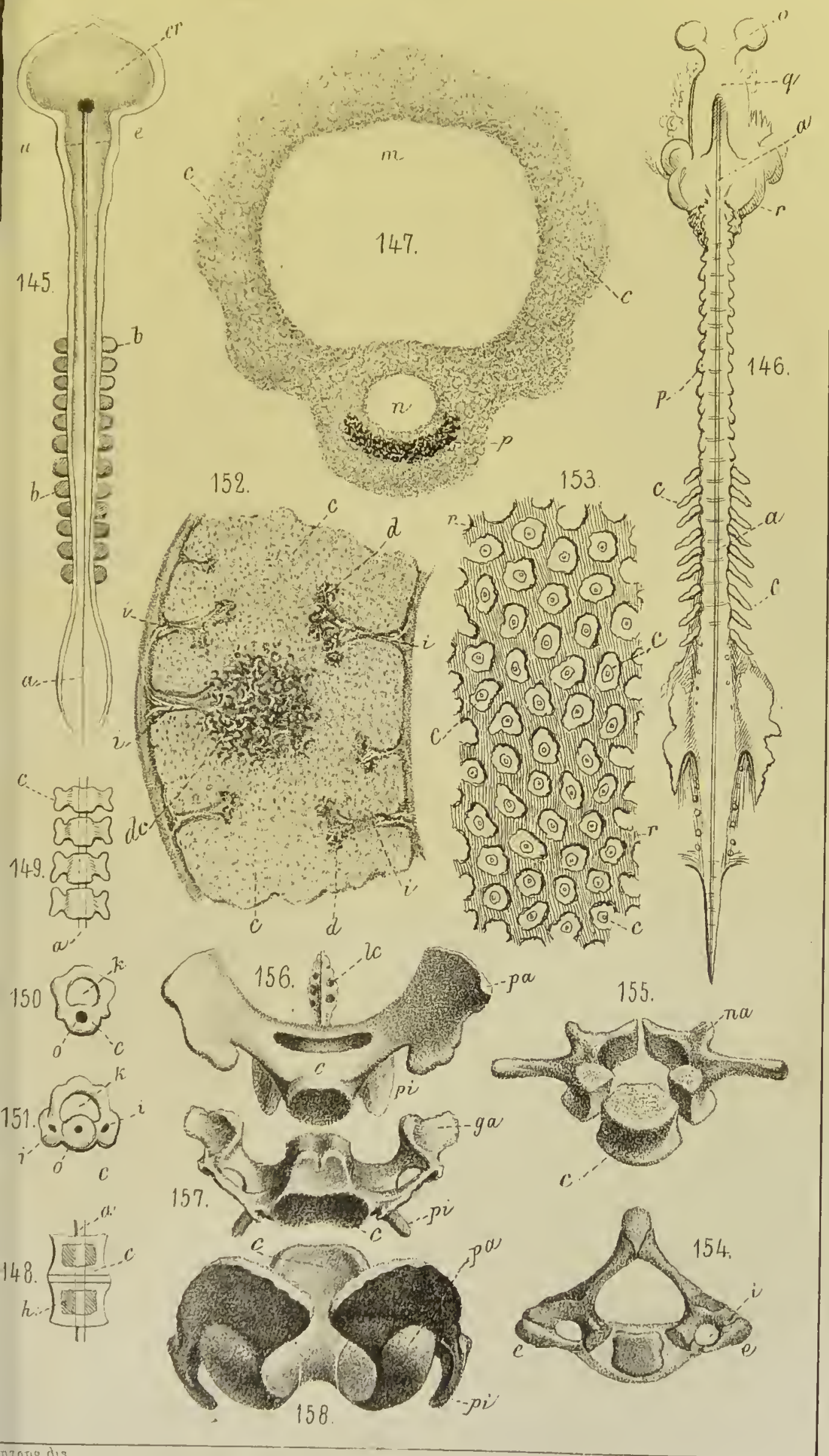

Lit Sterger 

TA VOLA DEGIII I OUINTA 
SEGUITO IELLO SVILUPPO DELLO SCHELETKO

Fig. 159 - Osso frontale d'un feto uniano alla fine del terzo mese (si vede ancora diviso nella linea mediana); $a$ apofisi orbitaria esterna; $a^{\prime}$ apofisi orbitaria interna.

1) 160 - Osso temporale d'un bambino coll' anello timpanico $a$.

" 161 - Etmoide d'un bambino ; $a$ selto mediano carlilaginoso; $b$ apofisi cristagalli; $d$ cellule etmoidali (cornetti e lamina papiracea) coperte e riunile da una guaina fibrosa piultosto spessa, Ia quale iu $f$ forma un cappuccio a ridosso dell' apofisi cristagalli ; oo lamine cribrose.

1) 162 - Occipitale d'un feto di circa quattro mesi (veduto dall' interno); $s$ 'squama inferiore originata da due punti d'ossificazione nella guaina fibrosa del cranio; ce condili; a doccia basilare ancora separata dai condili ; $i$ cartilagine che separa la squama dai condili.

" 163 - Vomere d'uı bambino in forma d' una navicella.

D) 16/ - Testa di un embrione umano di tre sellimane; $a$ grandi emisferi ; $b$ solco mediano; $c$ terzo ventricolo; $d$ corpi quadrigemelli; $e$ processo mediano frontale ; $f$ processo mascellare superiore del primo arco branchiale; $h$ lingua ; $i$ mascella iıferiore; $l$ regione superiore del collo; $m$ occhio.

1) 165 - Estremità cefalica d' un embrione umano di circa tre settimane (veduta dal davanti, ed ingrandita sei volte); $p$ prosencefalo ; $m$ mesencefalo ; $o$ occhio $h$ apertura orale; $p r$ processo mascellare superiore; 1, 2, 5, 4 archi viscerali.

n) 166 - Testa d'un embrione umano di circa cinque sellimane veduta dal davanti (ingrandita quatlro voltc); 
a occhio ; ok processo del primo arco branchiale $o$ mascella superiore; z lingua; $g$ mascella inferiore, $f$ processo frontale medio; $n$ narici; $i$ fovea udiliva o fessura branchiale tra il primo e secondo arco $; v$ grandi emisferi ; $m$ mesencefalo.

Fig. 167 - Cranio e faccia d' un feto umano veduti di sotto $; j$ processo di Mekel; $i$ mascella inferiore ; $q$ osso ioide; $l$ cerchio timpanico in mezzo al qualc si vedono gli ossicini dell'udito; $p$ gran corno dell'ioide. " 168 - Mascclla inferiore sinistra d' un bambino, nclla quale si osserva la disposizione di tutti gli alveoli dei denti permanenti $p p p$; l'ultimo molare, che nella figura è il tcrzo, trovasi alla base dell'apofisi montante; $d d$ denti di latte; $c$ canale dentario.

„169 - Sviluppo degli arti nelle prime otto settimane -

$A$ papilla primitiva con uno strozzamento mediano $s$ e con un orlo sporgente schiacciato $c$.

$B$ la stessa più sviluppata con l' inizio dei solchi interdigitali $d d$ nell' orlo.

$C$ la stessa in cui si vedono già distinte le cinque dita $l$, che per altro sono ancora riunite da una specie di membrana interdigitale $m$.

$D$ la stessa con dita già scparatc.

Nelle figurc C c D si osserva già la distinzione del braccio, avambraccio e mano.

1) 170 - Sterno interamente cartilagineo con solco mediano $s$; quale residuo della primiliva divisione in due metà laterali.

》171 - Sterno con cinque punti d'ossificazione $\int \mathrm{f}$.

" 172 - Osso iunominato d'un bambino diviso nelle tre parti cioc̀: $\boldsymbol{\Lambda}$ ilco, veduto dalla sua faccia esterna; $\mathrm{B}$ ischio; $\mathrm{C}$ pubc.

"17.5 - Tibia destra d'un feto, che in $a$ e $b$ é ancora carLilaginca; in $c$ e $d$ si lia una piastrina ossca coperta di cartilagine. 


T A V O L A DEC I II A SEST A 


\section{SVILUPPO DEL TUBO DIGERENTE E SUOI ANNESSI}

Fig. 174. - Intestino d' un embrione di cane reduto di sotto, secondo Bischoff; $a$ archi viscerali o branchiali; $b$ inizio delle fauci e della laringe ; $c$ polmoni ; $d$ ventricolo; $k$ fegato; $g$ parete della vescicola ombelicale.

1175-Embrione di cane di venticinque giorni (ingrandito due volte). La parete anteriore addominale è tolta ; perciò la cavità addominale vedesi aperta più di quello che lo è realmente in questa età. Il cuore è posto a nudo $a$ fosse nasali $; b$ occhio $; c$ mascella inferiore (primo arco branchiale); $d$ secondo arco branchiale; $e$ atrio destro del cuore; $f$ atrio sinistro del cuore; $g$ ventricolo destro; $h$ ventricolo sinistro; $i$ aorta; $l k k$ lobi del fegato che abbracciano il lume della vena omfalo-mesenterica; $m$ intestino dove passa nel dotto omfalo-meseraico; $n n$ parete della vescicola ombelicale; $o$ corpo del Wolff; $p p$ allantoide; $q$ arto anteriore; $r$ arto posteriore.

" $176-A B C$. Schemi per lo sviluppo del tubo intestinale. Nelle Fig.e $B$ e $C$ sono trasportate a destra le parti dell' intcstino le quali propriamente si troverebbero nel piano medio dell' addome; $m$ stomaco nelle sue successive forme e posizioni; $d$ duodeno; $o$ dotto omfalo-meseraico; $w$ anse intestinali; $b$ cieco; $c$ colon; $c^{1}$ colon trasverso; $c^{2}$ colon discendente; $f \mathrm{~S}$ iliaca; $e$ intestino retto.

\ 177 - Estremità caudale d'un embrione in cui si osserva l' apertura inferiore $u$ dell'intestino comune al seno uro-genitale; $c$ funicello ombelicale ; $a$ arti posteriori; $s$ coccige. 
Fig. 178-179-ABCD-Stadii successivi di sviluppo ¿del fegato dalla parete del canale digerente.

b) 180 - Visceri toracici ed addominali di un embrione umano di dodici settimane (in grandezza naturale); $v$ cieco col processo vermicolare che trovasi proprio sotto del fegato e quasi nella linea mediana ; $f$ fegato che occupa anteriormente tulta la cavità addominale, ne tiene assai distese le pareti ed occulta quasi tutto il pacchetto intestinale.

》 181 - A B C D-Stadii successivi di sviluppo del polmone. Formazione delle ramificazioni bronchiali e delle cellule polmonari.

1) 182 - Apparecchio degli archi e fessure branchiali di un embrione umano di circa trentacinque giorni, secondo Coste (la cavità della faringe è aperta e presenta la sua faccia interna); $m$ mascella inferiore; $h$ piccolo corno dell'ioide; $b$ archi viscerali inferiori; $g$ glottide; $p$ polmoni; $f$ fegato; $e$ stomaco; $i$ intestino tenue.

183-184-185 - Sviluppo del peritoneo viscerale e sue appendici (studiato in sezioni verticali antero-posteriori dell'addome); 183. Mesogastrio dopo che lo stomaco si è disposto trasversalmente; 184 . Formazione del grande omento; 185 . Stato definitivo.

Spiegazione delle cifre e leltere di queste tre figure; 1 lamina inferiore del meso-gastrio ; 2 lamina superiore (disegnata in punti); $m$ grande curvatura del ventricolo; $p$ piccola curvatura; $g$ legamento gastro-epatico; $s$ mesocolon trasverso; $t$ colon trasverso; $d$ intestino tenue; $m$ mesenterio $; h$ grande omento (costiluito da quattro lamine e nel suo principio da sei) $b$ peritoneo parietale anteriore; $\boldsymbol{P}$ pancreas.

La figura 185 può anche servire a spiegare lo stato primilivo del meso-gastrio prima che lo slomaco si 


$$
\text { - LII - }
$$

disponga trasversalmente; la grande curvatura dello slomaco $(m)$ ed il fondo della borsa meso-gastrica stanno allora a sinistra ed il disegno deve considerarsi come una sezione orizzonlale. 


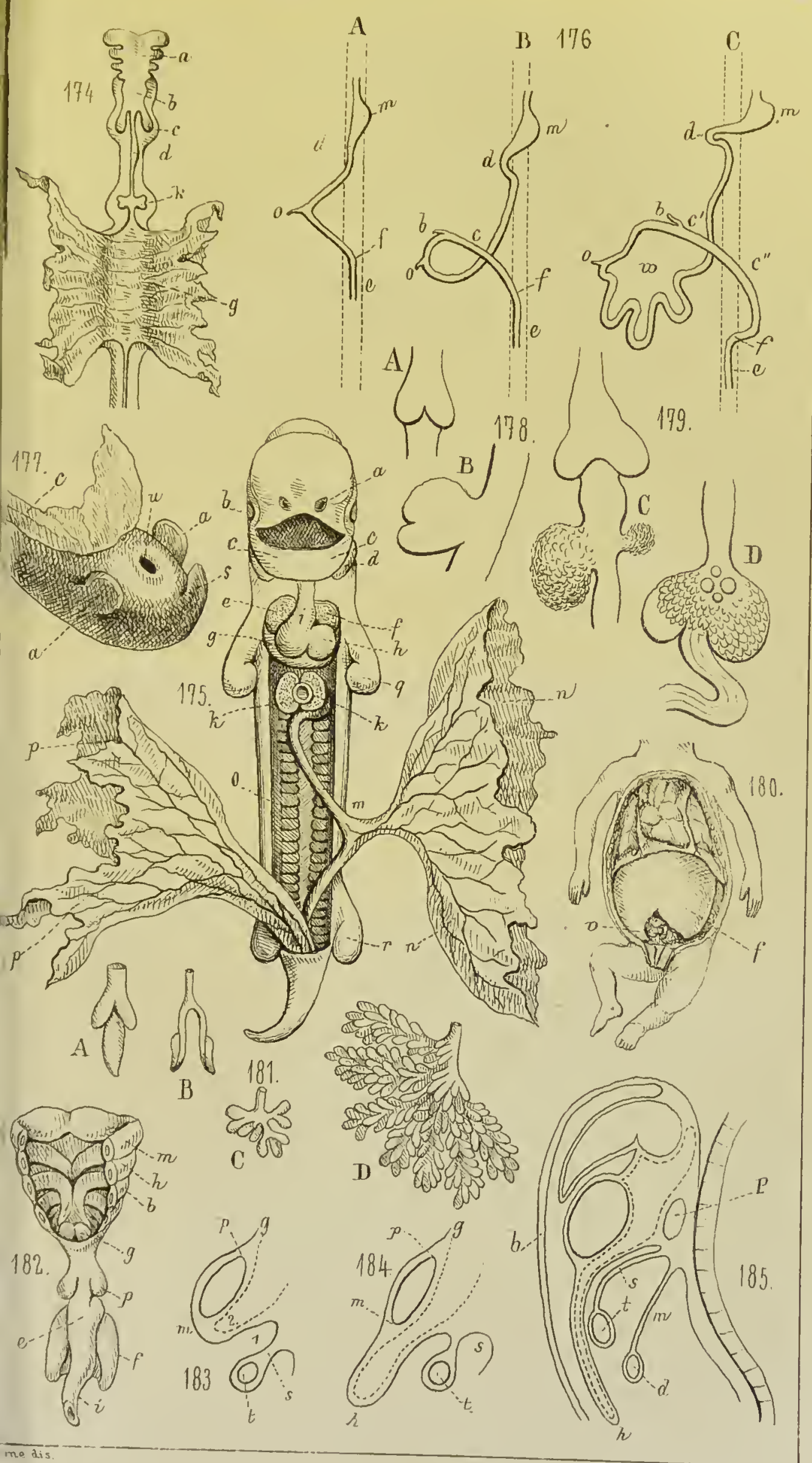



T A V OLA DECI II A SET TIIII A 


\section{SVILUPPO DELL'APPARECCHIO URO-GENITAI.E}

Fig. 186. - Organi uro-poetici e genitali femminili d' un embrione di bue lungo $11 / 2$ " (ingrand. del doppio); $w$ organi del Wolff; wg condotto dello stesso col cordone del Müller ; $i$ legamento inguinale; $o$ ovaja; $r$ rene; $r s$ relle succenturiato.

1) 187-Rene vero e rene succenturiato d'un embrione umano di circa sette mesi (grandezza naturale) ; rrr renuncoli o lobi del rene; $u$ uretere; $r s$ rene succenturiato.

188-Sviluppo degli organi genito-urinarii in un embrione umano, secondo Coste; $c v$ corpo deI Wolff; $o$ ovaja (o testicolo); $c$ rene succenturiato; $r$ rene; $t$ deferente ; $v$ vescica urinaria; $g i$ intestino retto; $u$ uretere.

1) 189-Schema dello sviluppo dei genitali interni maschili; $S$ seno uro-genitale; $t$ testicolo; $e$ epididimo; $d$ deferente; $u$ utero mascolino; $m$ condotto del Müller.

"190-Schema dello sviluppo dei genitali interni femminili; $o$ ovaja; $p$ parovario; $t$ tromba faloppiana; $f$ fimbrie della tromba (con idatide); $u$ utero; $S$ seno urogenitale.

1) 191-Genitali interni d'un embrione umano di sesso femminile di circa tre mesi (un poco ingrandilo); $r$ legamento rotondo dell'utero; $v$ ventricolo ; $d$ duodeno; $c$ colon; $o$ ovaja; ve vescica.

) 192-Seno urogenitale ed annessi d' un embrione umano (grandezza naturale); $\mathbf{A}$-di un embrione di tre mesi; $\mathrm{B}$-di un embrione di quattro mesi: $\mathrm{C}$-di un embrione di sei mesi; $b$ vescica urinaria; $h$ uretra; $n g$ seno urogenitale; $g$ canale genitale (rudimento dell'utero e della vagina); $s$ vagina; $u$ utero. 
Fig. 195-Schema a spiegare la discesa dei testicoli: A - il testicolo in priucipio del canale inguinale; $\mathbf{B}$-il testicolo nello scroto; $h$ testicolo; $a$ rivestimento peritoneale dello stesso (membrana adnata testis); $c v$ canale inguinale colla dilatazione inferiore $v$, la quale più tardi diviene la tunica vaginale propria.

»194-195-196-197-198-199-200. Sviluppo dei genitali esterui.

Lettere delle figure 194-200; ug apertura del seno uro-genitale; $g$ pliclie genitali (piccole labbra o corpi cavernosi del pene); $s$ coccige; $f$ solco genitale; $e$ ghiande 0 clitoride; $a$ ano $h l$ plica genitale esterra (scroto o grandi labbra).

\section{SVILUPPO DEGLI ORGANI DI SENSO}

1) 201-Embrione di pollo; $d$ midollo allungato; $f$ talami ottici ; $g$ emisfero cerebrale anteriore; $i$ orecchio; $o$ occhio.

1) 202-Testa d' un embrione di pollo di quattro giorni ; $n$ fossa nasale; $o$ processo mascellare superiore del primo arco branchiale; $u$ mascellare inferiore 0 primo arco branchiale; $k$ secondo arco branchiale; $s p$ fessura coroideale; $f$ fauci.

1) 20.5-Sezioni longitudinali dell'occhio d'un embrione di pollo, secondo Remak;

A occhiod'un embrione di sessantacinque ore;

B id: id: di settanta ore;

$C$ id: - id: di quattro giorni ;

$h$ foglio cornco; $l$ lente, che in $\mathbf{A}$ ha ancora la forma di un sacco ed è unita al foglio corneo, mentre in B ed in C si è già isolata ma è ancora cava; o fossetta leuticolare; $r$ vescicola oculare primiliva introflessa; $u$ parte posteriore della vescicola oculare che in $\mathbf{A}$ ed in $\mathbf{B}$ sta in comunicazione col cervellomediante il ner- 
vo oltico ancora tubulare; $x$ inspessimento del foglio corneo alla periferia, cioè dove la lente si è staccata; gl corpo vitreo.

Fig. 204-Metà posteriore d'una sezione verticale equatoriale dell' occhio d' un embrione umano di quattro settimane (ingrandimento circa 30 diam.); a lamella esterna della vescicola secondaria oculare (coroidea); $i$ lamella interna della stessa (retina); g corpo vitreo; $g^{\prime}$ picciuolo del corpo vitreo nella fessura coroideale; $h$ avanzo della vescicola oculare primitiva.

1) 205-Sezione verticale antero-posteriore dell'occhio d'un embrione di bue lungo $9^{\prime \prime \prime}$ (ingrand. circa venti volte); $c c$ congiuntiva dellacornea; $e$ sostanza della cornea; $l$ lente; $g$ corpo vitreo con vasi sanguigni; $r$ retina; $p$ coroidea; $s c$ sclerotica e parte vascolare della coroidea; $m m$ muscoli retti (superiore ed inferiore).

„206-Sviluppo della lingua, dell' ioide e della glottide in un embrione umano dicirca quaranta giorni, secondo Coste; $m$ mascella inferiore; $h$ ioide; $g$ glottide; $b$ trachea. 


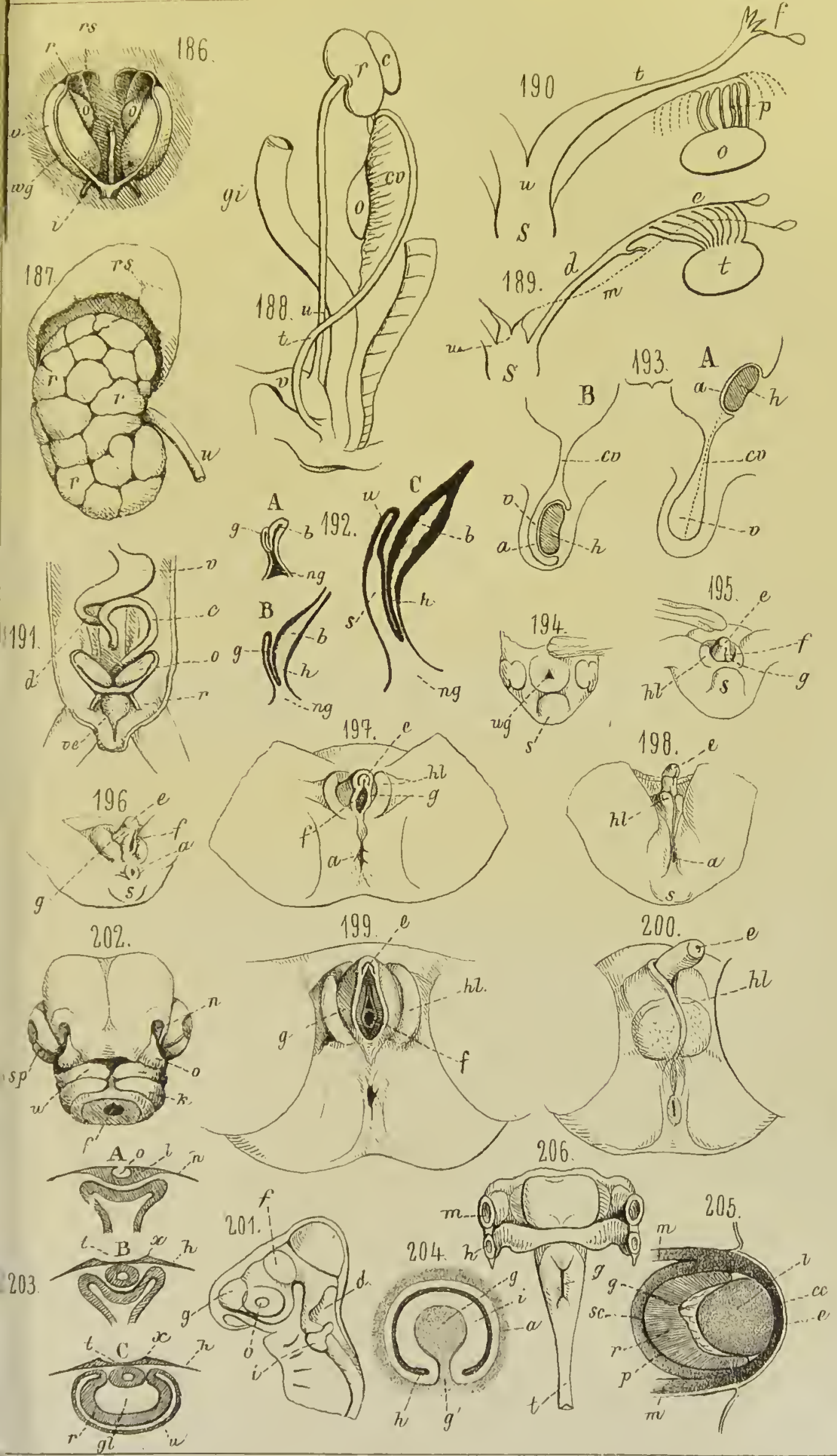



T A VOLA DE G I II A OTTA VA 
SVILUPPO DELL' APPARECCHIO VASCOLARE

Fig. 207-Areagerminativadi un coniglio con embrione veduto dalla faccia addominale, col primo sistema vascolare perfeltamente sviluppato, secondo Bischoff; aa iena 0 seno terminale; $b$ ramo anteriore della vena omfalo-meseraica ; $c$ ramo posteriore della stessa; $g$ cuore già piegato ad ansa; "Si vedono le due reti vascolari (arteriosa e venosa) di cui si parla nel testo a pag. 118 e seg. "

1) 208-Estremità cefalica di un embrione di pollo in principio del secondo giorno d'incubazione, veduta di sotto, secondo Remak; (ingrandita) ; $m$ tubo midollare; $h$ cuore ancora reltilineo; $h^{\prime} h^{\prime}$ venne sboccanti nel cuore.

1) 209-Estremità cefalica di un embrione di pollo verso la trentesimasesta ora d'incubazione veduta di sotto (ingrandita); $a$ bulbo arterioso; $k$ rigonfiamento ventricolare del cuore; $h^{\prime} h^{\prime}$ vene sboccanti nel cuore.

210-Cuore d'un embrione di pollo del terzo giorno d'incubazione veduto da destra ed ingrandito ; $a$ bulbo dell'aorta $a^{\prime} ; k$ rigonfiamento ventricolare sporgente verso destra; $v^{\prime}$ rigonfiamento corrispondente agli atrii a sinistra e posteriormente.

1) 2/1-Guore di un embrione di coniglio di dieci giorni: in A veduto d'avanti, in $\mathbf{B}$ veduto di dietro, secondo Bischoff (ingrandito); a bulbo arterioso da cui si stacca il tronco arterioso comune; $r$ ventricolo destro; $l$ ventricolo sinistro: vo vene omfalo-mesenteriche sboccanti nel cuore; $\boldsymbol{r}^{\prime \prime}$ orecchietta destra; $l^{\prime \prime}$ orecchietta sinistra.

12 212-Cuore di un embrione di cane; in A veduto dal davanti; in B veduto di dietro, secondo Bischoff. Que- 
sto cuore si trova in un grado di sviluppo più avanzato dell'antecedente; $a$ bulbo arterioso; $r$ ventricolo destro; $l$ ventricolo sinistro; $r^{\prime \prime}$ orecchietta destra; $l^{\prime}$ orecchielta sinistra; $e$ canale auricolare; $v$ foce renosa nel cuore.

Fig. 215-Cuore di un embrione umano lungo $7{ }^{\prime \prime}$ (ingrandito qualtro volte); $n$ ventricolo destro ; $l$ ventricolo sinistro ; ta tronco arterioso che incomincia a dividersi in due canali al segno ${ }^{*}$; $r$ orecchietta destra; $l$ orecchietta sinistra.

"2/4-Sezione orizzontale sulla base del cuore d' un enbrione umallo lungo $9^{\prime \prime \prime} ; l^{\prime \prime} r^{\prime}$ residui degli atrii esportati aderenti alla base dei ventricoli; $i$ margine dell'orificio atrio-ventricolare; $v$ valvole atrio-ventricolari in via di sviluppo; o fessura compresa fra $\mathbf{i}$ margini della valvola; $a$ aorta; $a$ arteria polmonare.

"215-A B C D E-fig. ${ }^{\text {s }}$ schematiche per meglio chiarire lo sviluppo del cuore dalla sua forma semplice tubulare rettilinea, come si vede in $\mathbf{A}$, alla forma perfetta che si osserva in $\mathbf{E}$ : 1 estremità arteriosa che corrisponde al bulbo arterioso e da cui si distaccano le arterie $a a$; 2 ventricolo o cuore vero comunicante in sopra col bulbo arterioso ed in sotto coll'estremità venosa 3 , in cui sboccano le due vene $v v$. In $\mathbf{E}$ le lettere $a a$ e $v v$ ed i numeri 125 corrispondono come nelle altre figure; $t a$ tronco arterioso; $r$ bulbo arterioso ; ${ }^{*}$ solco fra il bulbo ed il ventricolo; $r^{\prime}$ e $p$ orecchiette.

"216-Testa d'un embrione di cane veduta davanti ed ingrandita, secondo Bischoff; $a$ emisfero; $b$ occhio; $c$ mesencefalo; $d$ mascella inferiore; $e$ processo del primo arco branchiale che forma la mascella superiore; $f f^{\prime} f^{\prime \prime}$ secondo, terzo e quarto arco branchiale; $g$ atrio destro lel cuore; $h$ atrio sinistro del cuorc; $k$ ventri- 
colo destro; $i$ ventricolo sinistro; $l$ aorta con tre paia di archi aortici.

Fig.217-Estremità posteriore d'un embrione di cane coll'all' allantoide sviluppata e rovesciata in basso; $a$ alIantoide; $b$ estremità caudale dell'intestino; $c c$ aorte primitive che si ramificano sull'allantoide; $d d$ vene ombelicali che decorrono ai margini della parete addominale; $v$ vescicola ombelicale.

1218-Figura schematica della struttura della placenta, secondo Carpenter; $a a$ parete dell' utero; $b b$ seni della stessa in cui penetrano i villi vascolari vvv del chorion.

1) 219-Estremità d'un villo della placenta a forte ingrandimento, secondo Carpenter; a membrana esterna del villo; $b b$ strato cellulare esterno del villo; $d$ spazio tra i due inviluppi (materno e fetale) del villo; $f$ strato cellulare interno del villo (appartenente al chorion); $g$ ansa vascolare fetale.

" 220 - Cuore di un embrione umano lungo 2" 3 "' (ingrand. circa tre volte) l'atrio e l'orecchietta destra sono aperti; $s a$ setto degli atrii; $s a^{\prime}$ estremità 0 corno superiore di questo setto ; ve valvola d' Eustachio ; vc vena cava inferiore; $o$ ostio ventricolare destro; $r$ ventricolo destro; $r^{\prime}$ atrio destro aperto; $r^{\prime \prime}$ orecchietta destra aperta. 

T A V O L A D E G I II A N O N A 
Fig.221-Tronco arterioso col primo paio d'archi dell'norta ed indicazione del $2^{\circ}$ e $3^{\circ}$ paio.

)

222-Tronco arterioso con quattro paia d'archi dell'aorta ed indicazione del quinto arco.

223-Tronco arterioso coi tre archi aortici inferiori dai quali si sviluppano i vasi permanenti; indicazione dei due archi superiori che si obliterano.

224-Arterie permanenti nella loro forma primitiva ed indicazione delle parti degli archi branchiali che si 0 bliterano.

Lellere comuni alle figure anlecedenti; la tronco arterioso, dal $1^{0}$ al $5^{0}$ arco aortico; $a$ aorta; $p$ pulmonale $; p^{\prime} p^{\prime \prime}$ rami che vanno ai pulmoni; av radice dell'aorta che si oblitera; $s^{\prime \prime} s^{\prime \prime}$ succlavie ; $v$ vertebrale; $a x$ ascellare $; c$ carotide comune $; c^{\prime}$ carotide esterna; $c$ carotide interna; $a d$ aorta addominale.

„225-Primo sviluppo delle vene ombelicali mentre le omfalo-mesenteriche sono le vene pricipali del corpo.

226-Le vene omfalo-mesenteriche incominciano ad obliterarsi quando si sviluppano i primi vasi del fegato.

"227-228. Sistema venoso nel periodo in cui il circolo placentare è perfettamente sviluppato; om, in 225 , tronco dell'arteria omfalo-mesenterica; in 226 e 227 , omfalo-mesenterica permanente; in 228 , vena del sacco vitello intestinale; $o m^{\prime} a m^{\prime \prime}$ vena omfalo-mesenterica destra e sinistra; $u$ tronco delle vene ombelicali; $u^{\prime} u^{\prime \prime}$ vena ombelicale destra e sinistra; $d c d c$ dotti del Cuvier; ji giugulari; $c c$ cardiıali; l fegato; ha vene epatiche adveenti; $h r$ vene epatiche reveenli; $m$ mesenterica; da dotto venoso dell'Aranzio; $c i$ cava inferiore ; $p$ vena porta; $l$ vena lienale; $m$ me- senterica superiore. 
Fig. 229-Schema a dimostrare le vene maggiori del corpo nel tempo che si sviluppa la circolazione placentare '́nell'uomo rerso la quarta seltimana di gestazione); $v$ seno veno:o comune; $d c$ dolto del Cuvier; $j$ giugulare primiliva; $j i$ giugulare interna; $s$ succlavia; $c$ cardinale; $h$ cstremilà caudale della stessa, che in seguito diventa irogastrica; $c r$ crurale; $c i$ cava inferiore; om omfalo-mesenterica; $u$ ombelicale; $u^{\prime}$ tronco della slessa che va al fegato.

"250-25\%. Schemi a dimostrare la formazione dei sistemi della vena cava superiore ed inferiore.

Fig. 230. Veduta posteriore del cuorc e delle vene nel tempo in cui esistono due vene cave superiori; $c s$ cava superiore sinistra che riceve le vene del cuore; $c d s$ tronco della cardinale sinistra; $c d$ cava superiore destra; ad anonima destra (che in principio era l'origine della giugulare destra); as anonima sinistra (tronco di congiunzione fra le due giugulari primitive; az azygos (originariamente tronco della cardinale destra); $j i$ giugulare interna; $j c$ giugulare esterna; $s$ succlavia; $c$ parte mediana oblitcrata delle venc cardinali; $v p$ vene vertebrali posteriori sorte invece delle cardinali, nelle quali sboccano le vene lombari e le intercostali; ha tronco della emi-azygos (ramo di congiunzione fra le due vertcbrali); ci cava inferiore; il iliaca comunc (originariamente tronco di congiunzione della cava colla cardinale); $c r$ cruralc; $h$ ipogastrica (originariamente fine della cardinale). Fig. 231-Veduta posteriore del cuore c dei tronchi venosi permanenti con indicazione della scomparsa della vena cava superiore sinistra; cs cava superiore sinistra obliterata; $j c$ giugulare comunc; $i$ intercoslale suprema; hus emi-azygos superiore; hai emi-azygos inferiore; ha tronco della cmi-azygos; sc seno coronario in cui sboccano le grose vene del cuore (estremità della cava superiore sinistra). 
Fig. 252-Fegato di un feto maturo ( $4 / 6$ della grandezza naturale) veduto dal di sotto. Sono levale la parle superiore del lobo dello Spigelio, le parli che limilano il solco sinistro ed una parte del lobo destro; u tronco dell'ombelicale; $u^{\prime} u^{\prime \prime}$ rami della stessa pel lobo sinistro e pel lobo destro; $d v$ dotto venoso dell'Aranzio; $p$ vena porta; $c i$ cava inferiore aderente al fegato; $h$ vene epatiche; $f$ cistifellea ; $c$ cava inferiore fuori del fegato.

)

235-Cuore di un embrione umano maturo veduto dal davanti ed un poco da sinistra; cs cava superiore; $a$ arteria anonima; $c$ carotide sinistra; $s$ succlavia sinistra; $a o$ estremità dell' arco dell'aorta; $d a$ dotto arterioso del Botallio; $a d$ aorta toracica; $a p$ arteria pulmonale sinistra; $p$ vena pulmonale sinistra.

1) 234-Schema della circolazione fetale ; 1 cordone ombelicale; 2 placenta; 5 vena ombelicale clie si divide in tre rami ( $a a$ vene adveenti pei lobi del fegato e $d$ dotto dell'Aranzio); 4 vena porta; 5 vena cava inferiore; 6 atrio destro del cuore; 7 atrio sinistro del cuore; 8 ventricolo sinistro del cuore; $g$ arco dell'aorta; ${ }^{10}$ aorta discendente ; 11 arterie ombelicali; 12 ventricolo destro; 15 arteria polmonare; 14 dotto arterioso del Botallio; 15 cava discendente.

1) 235 -Fetolino umano lungo $7^{\prime \prime \prime}$. I contorni del corpo sono appena delineati schematicamente per dare un'idea dei rapporti di dimensione fra il cuore ed il corpo in questo periodo della vita intrauterina.

\section{A VVERTENZA}

Sono originali le figure $34-35-36-62-63-64-65-66-$ $77-98-1$
$187-215$. 
Tav. XIX.

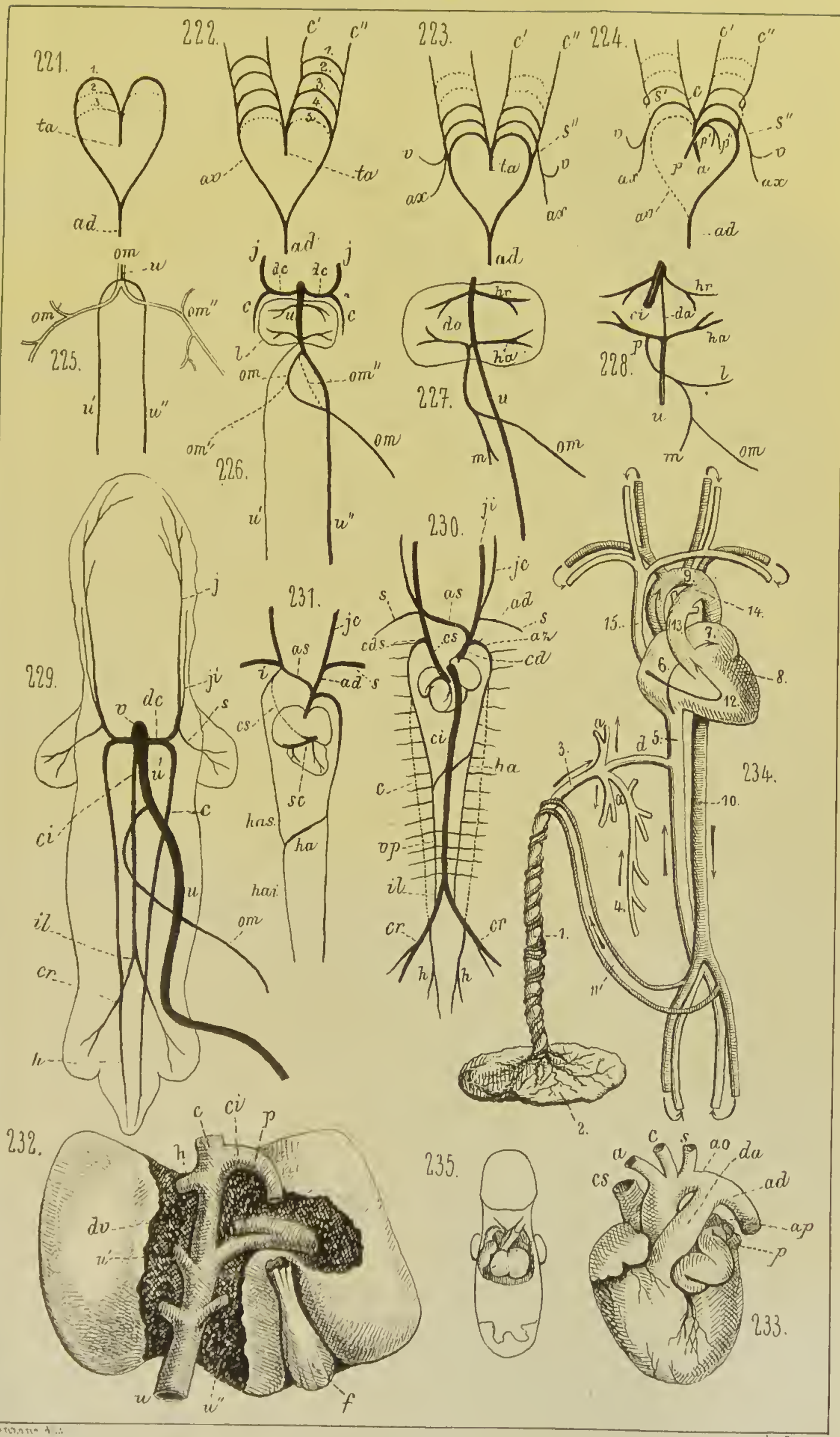







